\title{
Asymmetric Synthesis of Azepine-Fused Cyclobutanes from Yne-Methylenecyclopropanes Involving Cyclopropanation/C-C Cleavage/Wagner-Meerwein Rearrangement and Reaction Mechanism
}

\author{
Chen-Long Li, and Zhi-Xiang Yu*
}

Beijing National Laboratory of Molecular Sciences (BNLMS), Key Laboratory of Bioorganic Chemistry and Molecular Engineering, College of Chemistry, Peking University, Beijing, 100871,

$$
\text { China }
$$

E-mail: yuzx@pku.edu.cn

\section{Contents}

I. Scheme for Synthesis of Substrates

II. Racemic Product Synthesis

III. X-ray Crystal Analysis

Page S4

IV. DFT Calculations

V. References

VI. NMR Spectra

VII. High Performance Liquid Chromatography (HPLC) Data 
I. Scheme for Synthesis of Substrates
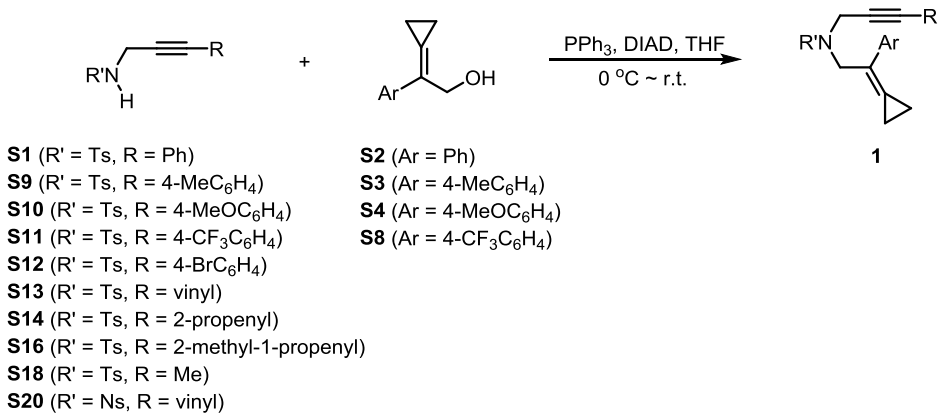

S1 $\left(R^{\prime}=T s, R=P h\right)$

S9 $\left(R^{\prime}=T s, R=4-M^{\prime} C_{6} \mathrm{H}_{4}\right)$

S10 $\left(R^{\prime}=\mathrm{Ts}, \mathrm{R}=4-\mathrm{MeOC}_{6} \mathrm{H}_{4}\right.$

S11 $\left(R^{\prime}=\mathrm{Ts}, \mathrm{R}=4-\mathrm{CF}_{3} \mathrm{C}_{6} \mathrm{H}_{4}\right)$

S12 ( $\left.\mathrm{R}^{\prime}=\mathrm{Ts}, \mathrm{R}=4-\mathrm{BrC}_{6} \mathrm{H}_{4}\right)$

13 $\left(R^{\prime}=\right.$ Ts, $R=$ vinyl)

$S 14\left(R^{\prime}=T s, R=2\right.$-propenyl)

$S 16\left(R^{\prime}=T s, R=2\right.$-methyl-1-propenyl)

$S 18\left(R^{\prime}=T s, R=M e\right)$

S20 ( R' = Ns, R = vinyl)

TBDPSO

S5

S6

S16

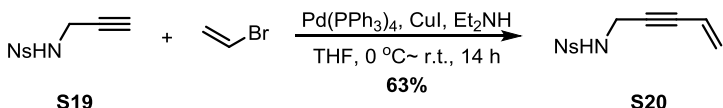

S20

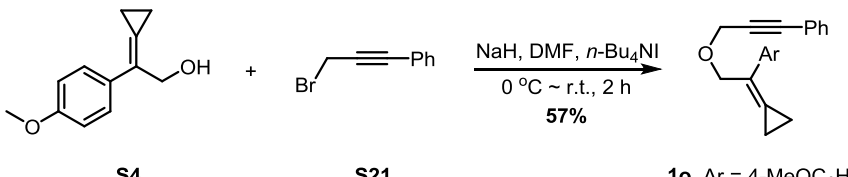

r.t. $=$ room temperature; $\operatorname{TBDPS}=t$-butyldiphenylsilyl.

Scheme S1 Synthesis of substrates 
II. Racemic Product Synthesis

\section{Racemic product synthesis}
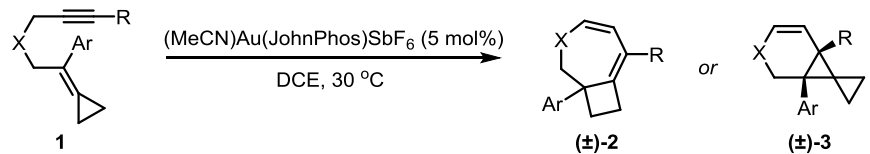

General procedure A: (5 mol \% catalyst)

General procedure of $\mathrm{Au}(\mathrm{I})$-catalyzed cyclization/rearrangement reaction: Under nitrogen, the commercially available $(\mathrm{MeCN}) \mathrm{Au}(\mathrm{JohnPhos}) \mathrm{SbF}_{6}(7.8 \mathrm{mg}, 10.1 \mu \mathrm{mol})$ was added to a flame-dried glassware containing $1(0.2 \mathrm{mmol})$. Anhydrous DCE $(4.0 \mathrm{ml})$ was added and the glassware was then immersed into an oil bath at $30^{\circ} \mathrm{C}$. The reaction was monitored by TLC. Upon completion, the reaction mixture was purified by flash column chromatography on silica gel to afford $( \pm)-2$ or $( \pm)-3$.

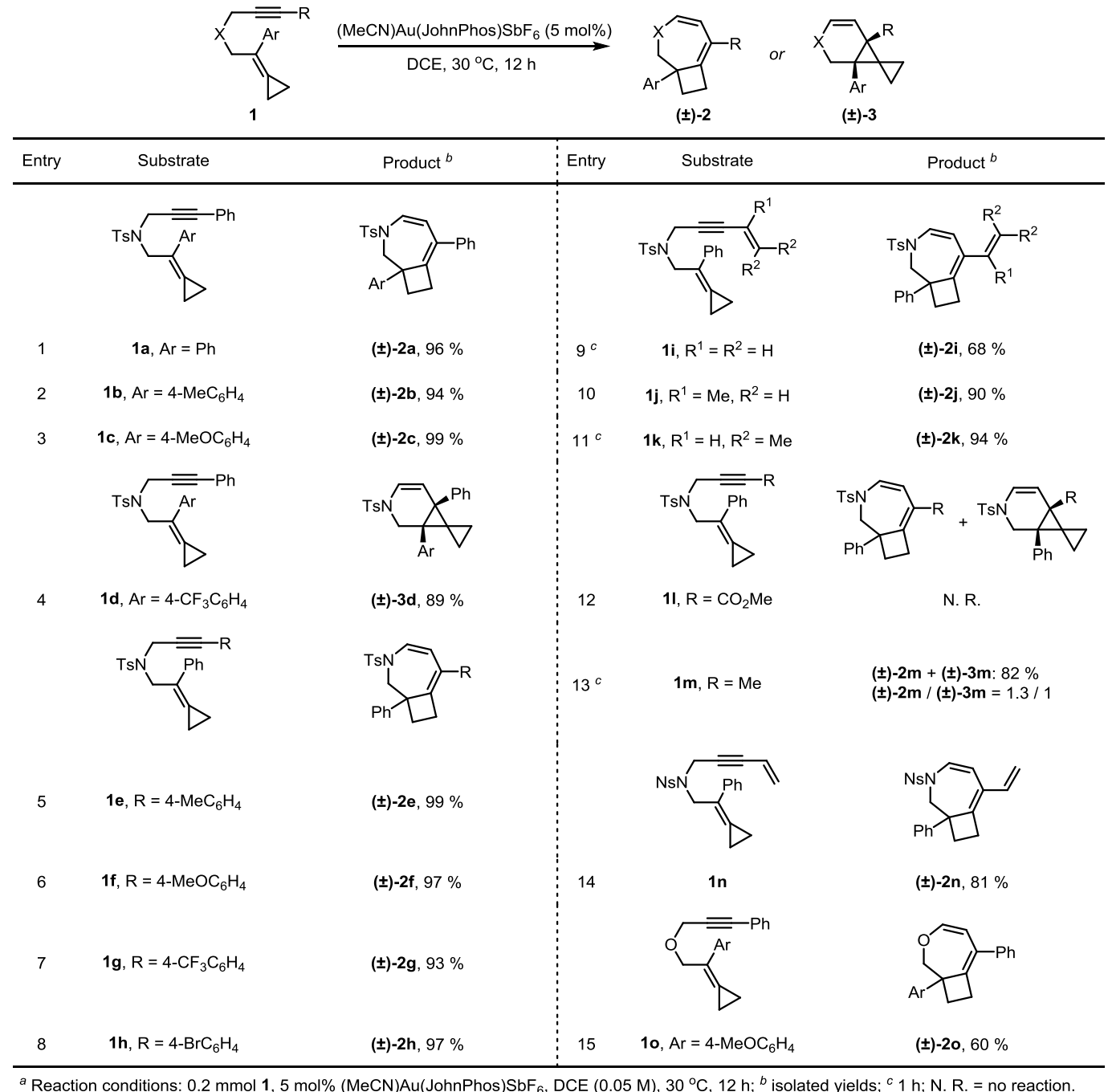

Table S1 ${ }^{a}$ Racemic product synthesis 
III. X-ray Crystal Analysis

Crystal compound ( \pm )-2a was obtained by adding $n$-hexane to its dichloromethane solution and then stilling for several days.

\section{Crystallographic Data of Compound ( \pm )-2a}

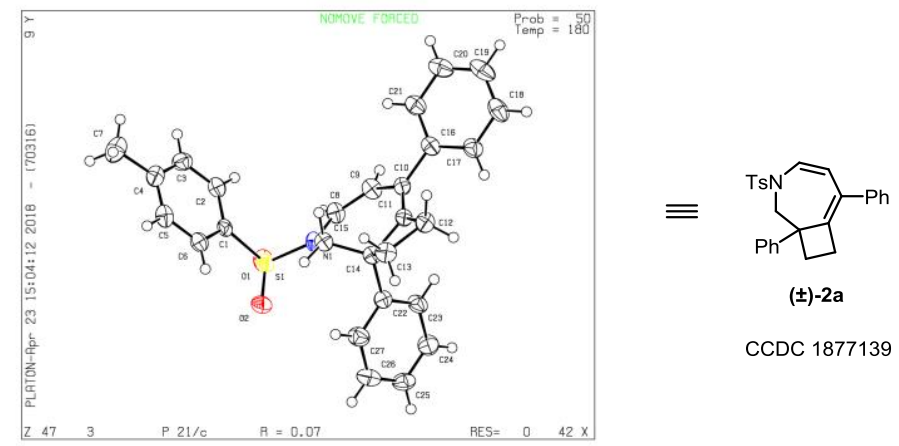

Table S2 Crystal data and structure refinement for Compound ( \pm )-2a.

\begin{tabular}{|c|c|}
\hline Identification code & 3 \\
\hline Empirical formula & $\mathrm{C}_{27} \mathrm{H}_{25} \mathrm{NO}_{2} \mathrm{~S}$ \\
\hline Formula weight & 427.54 \\
\hline Temperature/K & $179.99(10)$ \\
\hline Crystal system & monoclinic \\
\hline Space group & $\mathrm{P} 2{ }_{1} / \mathrm{c}$ \\
\hline $\mathrm{a} / \AA$ & $12.7314(8)$ \\
\hline $\mathrm{b} / \AA$ & 23.7964(15) \\
\hline $\mathrm{c} / \AA$ & $7.2381(4)$ \\
\hline$\alpha /^{\circ}$ & 90 \\
\hline$\beta /{ }^{\circ}$ & $90.855(5)$ \\
\hline$\gamma /{ }^{\circ}$ & 90 \\
\hline Volume $/ \AA^{3}$ & $2192.6(2)$ \\
\hline $\mathrm{Z}$ & 4 \\
\hline$\rho_{\text {calc }} \mathrm{g} / \mathrm{cm}^{3}$ & 1.295 \\
\hline$\mu / \mathrm{mm}^{-1}$ & 0.172 \\
\hline $\mathrm{F}(000)$ & 904.0 \\
\hline Crystal size $/ \mathrm{mm}^{3}$ & $0.2 \times 0.1 \times 0.05$ \\
\hline Radiation & $\operatorname{MoK} \alpha(\lambda=0.71073)$ \\
\hline $2 \Theta$ range for data collection $/{ }^{\circ}$ & 5.884 to 54.968 \\
\hline Index ranges & $-16 \leq \mathrm{h} \leq 16,-30 \leq \mathrm{k} \leq 29,-9 \leq 1 \leq 9$ \\
\hline Reflections collected & 21099 \\
\hline Independent reflections & $4899\left[\mathrm{R}_{\mathrm{int}}=0.0776, \mathrm{R}_{\text {sigma }}=0.0626\right.$ \\
\hline Data/restraints/parameters & $4899 / 0 / 281$ \\
\hline Goodness-of-fit on $\mathrm{F}^{2}$ & 1.007 \\
\hline
\end{tabular}


Final $R$ indexes $[I>=2 \sigma(I)] \quad R_{1}=0.0718, w_{2}=0.1452$

Final $\mathrm{R}$ indexes [all data] $\quad \mathrm{R}_{1}=0.0993, \mathrm{wR}_{2}=0.1552$

Largest diff. peak/hole / e $\AA^{-3} \quad 0.50 /-0.39$

Crystal compound $\mathbf{2 h}$ was obtained by adding $n$-hexane to its ether solution and then stilling for several days.

\section{Crystallographic Data of Compound 2h}

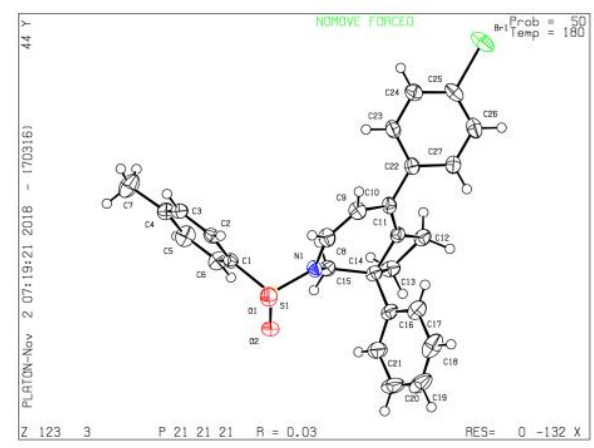

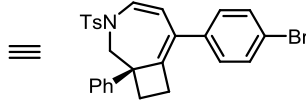

$2 \mathrm{~h}$

CCDC 1877140

Table S3 Crystal data and structure refinement for Compound $\mathbf{2 h}$.

\begin{tabular}{|c|c|}
\hline Identification code & 3 \\
\hline Empirical formula & $\mathrm{C}_{27} \mathrm{H}_{24} \mathrm{BrNO}_{2} \mathrm{~S}$ \\
\hline Formula weight & 506.44 \\
\hline Temperature/K & $180.00(10)$ \\
\hline Crystal system & orthorhombic \\
\hline Space group & $\mathrm{P} 2{ }_{1} 2_{1} 2_{1}$ \\
\hline $\mathrm{a} / \AA$ & $7.05990(10)$ \\
\hline $\mathrm{b} / \AA$ & $15.7538(2)$ \\
\hline$c / \AA$ & $20.8421(3)$ \\
\hline$\alpha /{ }^{\circ}$ & 90 \\
\hline$\beta /{ }^{\circ}$ & 90 \\
\hline$\gamma /{ }^{\circ}$ & 90 \\
\hline Volume $/ \AA^{3}$ & $2318.06(6)$ \\
\hline $\mathrm{Z}$ & 4 \\
\hline$\rho_{\text {calc }} \mathrm{g} / \mathrm{cm}^{3}$ & 1.451 \\
\hline$\mu / \mathrm{mm}^{-1}$ & 1.888 \\
\hline $\mathrm{F}(000)$ & 1040.0 \\
\hline Crystal size $/ \mathrm{mm}^{3}$ & $0.25 \times 0.12 \times 0.1$ \\
\hline Radiation & $\operatorname{Mo~} \mathrm{K} \alpha(\lambda=0.71073)$ \\
\hline \multicolumn{2}{|c|}{$2 \Theta$ range for data collection ${ }^{\circ} 4.686$ to 54.966} \\
\hline Index ranges & $-9 \leq \mathrm{h} \leq 9,-20 \leq \mathrm{k} \leq 20,-27 \leq 1 \leq 27$ \\
\hline Reflections collected & 53482 \\
\hline Independent reflections & $5320\left[\mathrm{R}_{\mathrm{int}}=0.0287, \mathrm{R}_{\text {sigma }}=0.0136\right]$ \\
\hline
\end{tabular}


Data/restraints/parameters $\quad 5320 / 0 / 290$

Goodness-of-fit on $\mathrm{F}^{2} \quad 1.032$

Final $R$ indexes $[I>=2 \sigma(I)] \quad R_{1}=0.0253, \mathrm{wR}_{2}=0.0659$

Final $\mathrm{R}$ indexes [all data] $\quad \mathrm{R}_{1}=0.0273, \mathrm{wR}_{2}=0.0669$

Largest diff. peak/hole / e $\AA^{-3} 0.77 /-0.61$

Flack parameter $\quad-0.0053(16)$ 
IV. DFT Calculations

1. The computed energy surface for the reaction of $\mathbf{1 a}$

2. Study of the rearrangement of the homoallylic cation

3. The computed energy surface for the reaction of the substrate used in Shi's work

4. The chemoselectivity of $\mathbf{1 m}$

5. Discussion of DFT functionals

6. The chemoselectivity of $\mathbf{1 d}$

7. Energy data

8. Cartesian coordinates of all stationary points

1. The computed energy surface for the reaction of $\mathbf{1 a}$

Here we give all data from different calculation methods for reaction of $\mathbf{1 a}$ in order to better understand factors affecting the reaction outcomes. As shown in Figure S1, the ring expansion (through TS3-Ph) has a lower activation energy than the direct [1,2]-hydride shift of INT2-Ph (through TS2-Ph) in $\Delta G_{\text {sol-DCE }}, \Delta G_{\text {gas }}$ and $\Delta H_{\text {gas. }}$ These data are consistent with the experiment observation that only 3-azabicyclo[5.2.0]nonadiene product (Pro2-Ph) is found.

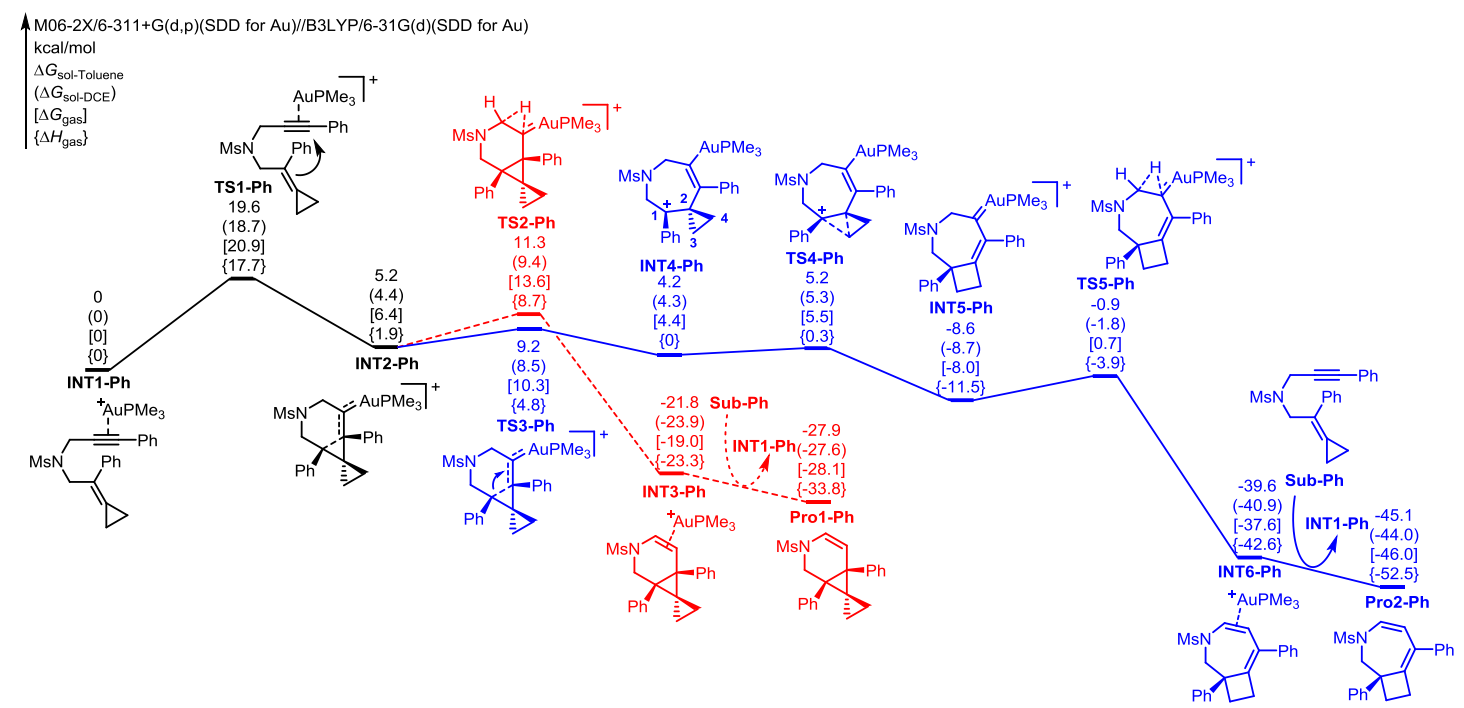

Figure S1 Energy surface for the reaction of 1a

2. Study of the rearrangement of the homoallylic cation

Relaxed scans of potential energy surfaces at B3LYP/6-31G(d) (SDD for Au) level were then performed to locate another homoallylic cation at C4 (Figure S2). During the scan of the potential energy surface, only the single-point energy of each structure could be obtained. Due to this, we give single-point energies in the gas phase ( $\left.\Delta E_{\mathrm{gas}}\right)$ to discuss processes.

As shown in Figure S2, with the length of C2-C3 bond in INT4-Ph decrease (from $1.68 \AA$ to 1.53 $\AA$ ), the $\Delta E_{\text {gas }}$ has an increase of $2.5 \mathrm{kcal} / \mathrm{mol}$. Although C2-C3 bond becomes shorter, the C2-C4 bond is the same (from $1.54 \AA$ to $1.53 \AA$ ). This can be attributed to the steric repulsion between the methylene group at $\mathrm{C} 4$ and phenyl group adjacent to $\mathrm{C} 2$. 


\section{Length of $\mathrm{C} 2-\mathrm{C} 4$ bond}
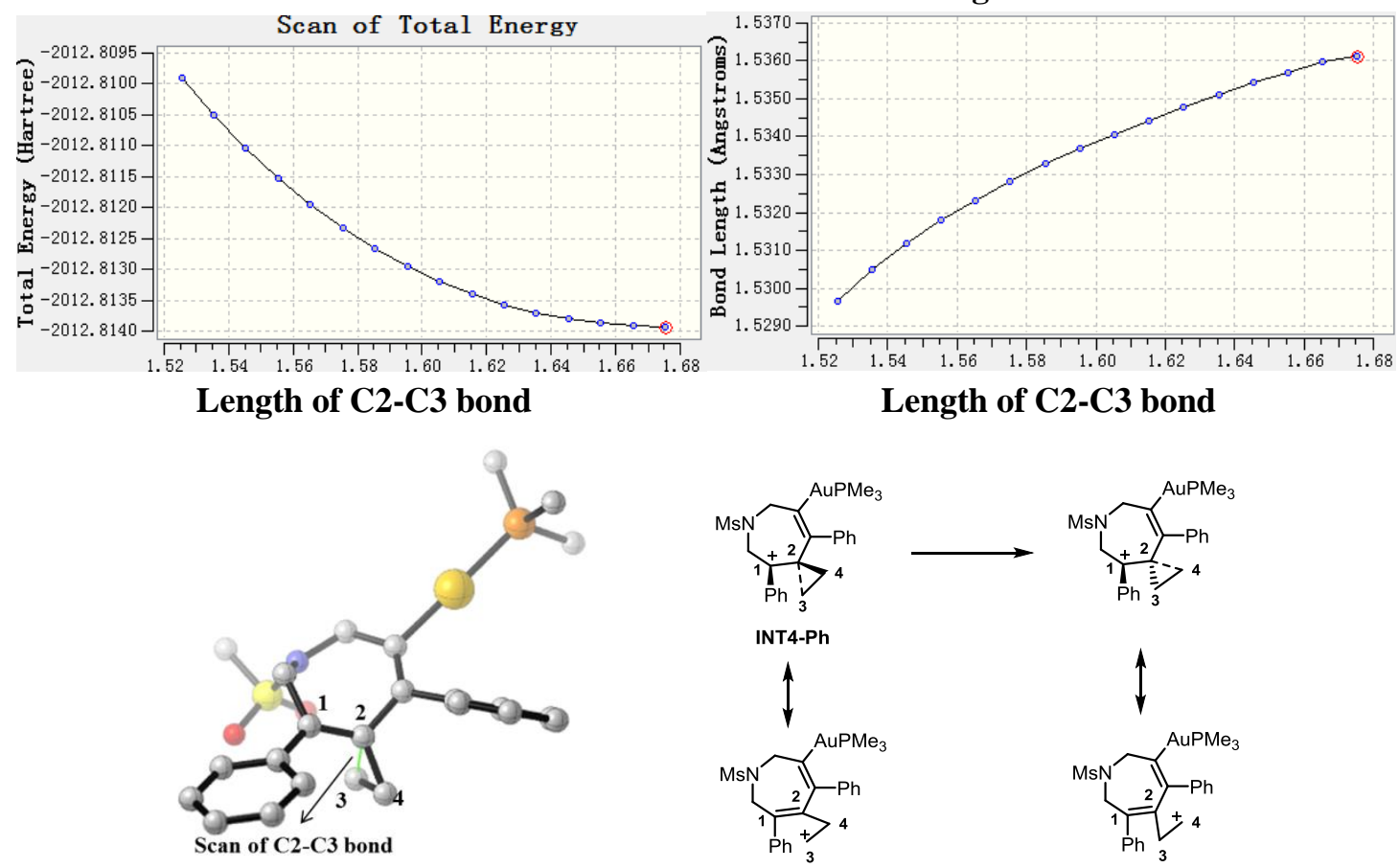

Figure S2 The rearrangement of the homoallylic cation and scan of $\mathrm{C} 2-\mathrm{C} 3$ bond

Based on the final structure in Figure S2, we sequentially scanned the length of C2-C4 bond. With its increase to $2.13 \AA$, the $\Delta E_{\text {gas }}$ is an increase of $15.7 \mathrm{kcal} / \mathrm{mol}$ (Figure S3). During the scan, the C2-C3 bond will change from $1.67 \AA$ to $1.63 \AA$, which is just a slight decrease. If we freeze the C2-C3 bond to $1.53 \AA$ at this scan, a similar result of $14.4 \mathrm{kcal} / \mathrm{mol}$ in $\Delta E_{\text {gas }}$ can be found (Figure S4).

From these two calculations, we can conclude that the formation of another homoallylic cation at $\mathrm{C} 4$ with the $\mathrm{C} 2-\mathrm{C} 3$ bond formation and the $\mathrm{C} 2-\mathrm{C} 4$ bond breaking in INT4-Ph has a rearrangement energy of $18.2 \mathrm{kcal} / \mathrm{mol}(2.5 \mathrm{kcal} / \mathrm{mol}+15.7 \mathrm{kcal} / \mathrm{mol})$. This process can be excluded because the Wagner-Meerwein rearrangement of INT4-Ph is faster, which has an activation energy of 1.0 $\mathrm{kcal} / \mathrm{mol}$. Therefore, there is no rearrangement of the homoallylic cation here.

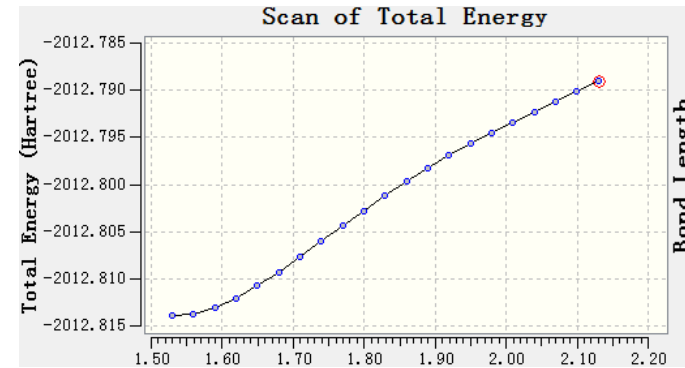

Length of C2-C4 bond
Length of C2-C3 bond

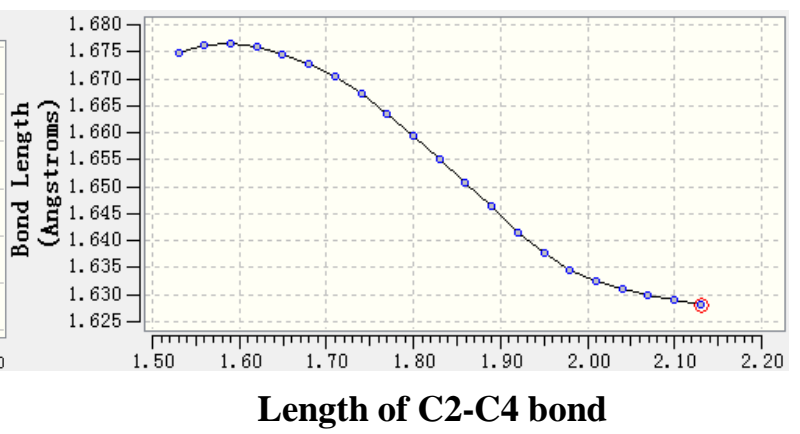




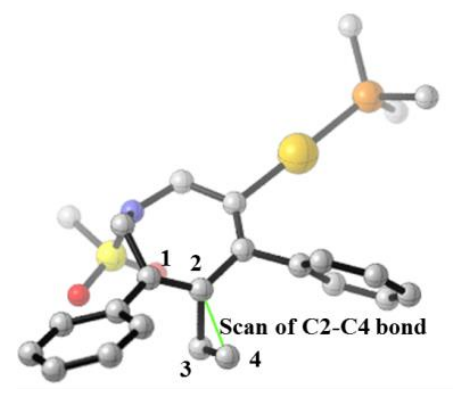

Figure S3 Scan of C2-C4 bond
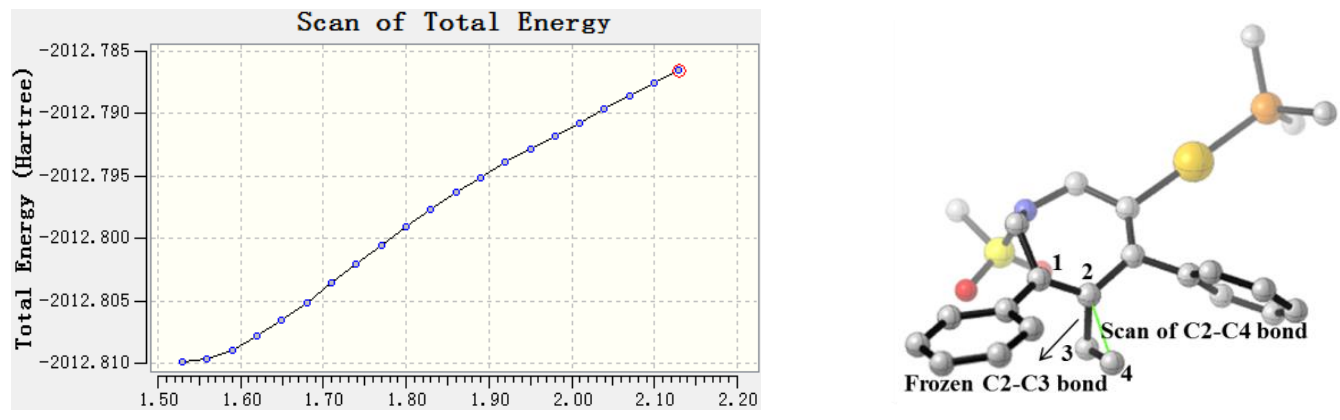

Length of C2-C4 bond

Figure S4 Scan of C2-C4 bond with a fixed C2-C3 bond

We also scanned the energy surface given in Figure S5. The energy rises to $17.6 \mathrm{kcal} / \mathrm{mol}$ with the $\mathrm{C} 2-\mathrm{C} 3$ bond decreasing and the $\mathrm{C} 2-\mathrm{C} 4$ bond increasing, which is also consistent with previous results.

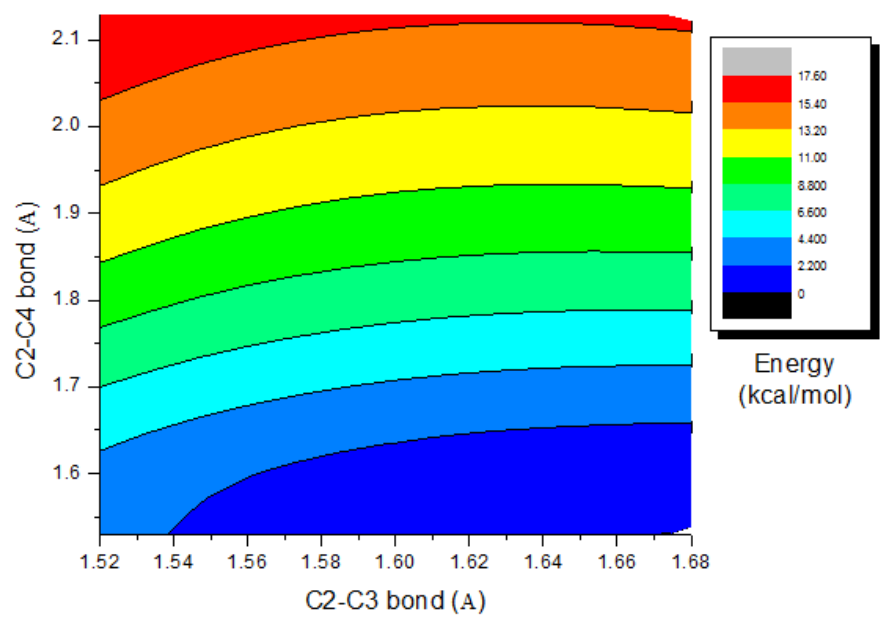

Figure S5 The scanned energy surface

3. The computed energy surface for the reaction of the substrate used in Shi's work

The full energy surface for MCP substrate without the aromatic group is given in Figure S6. Here we give all data from different calculation methods in order to better understand factors affecting the reaction outcomes. The ring expansion via TS3-H is disfavored compared to the direct [1,2]-hydride shift via TS2-H. The calculation results show that only cyclopropanation product (Pro1-H) will be observed in Shi's work, which is consistent with experiments. 


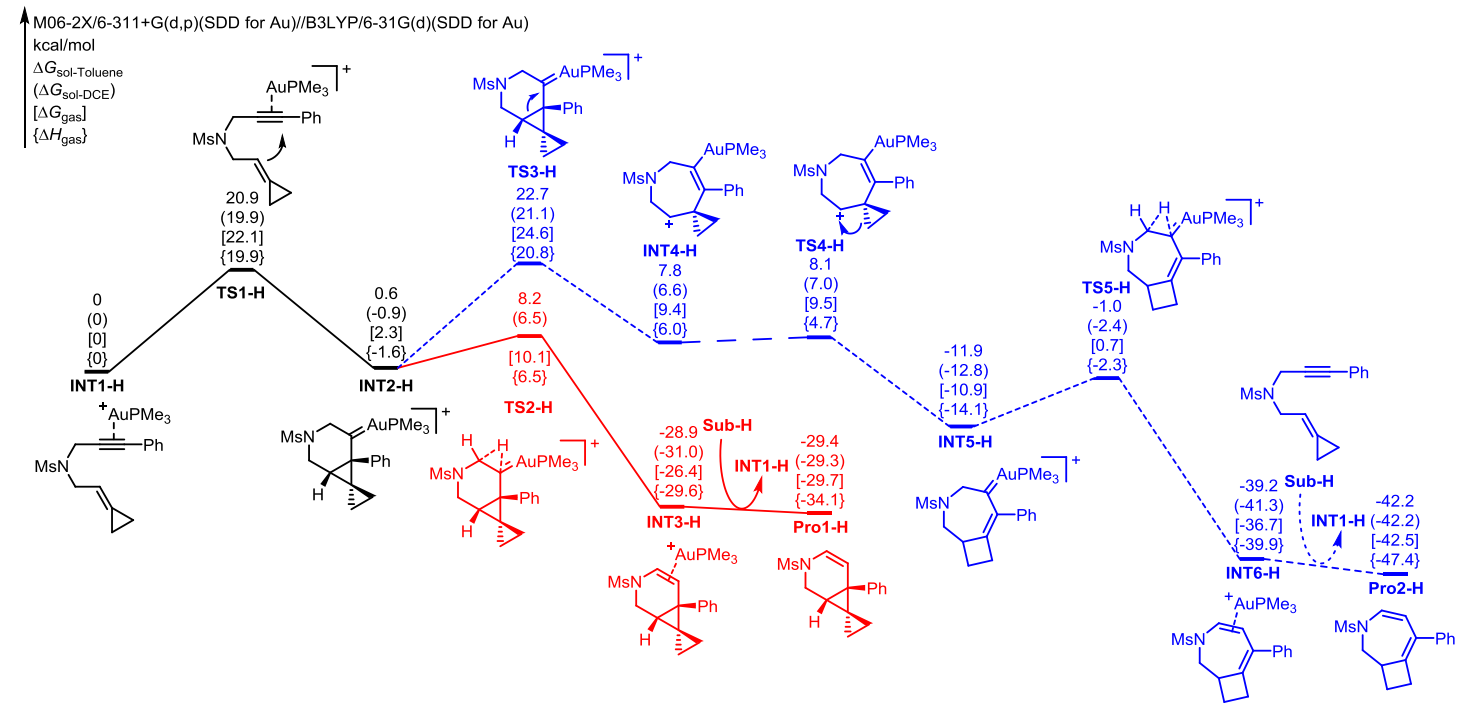

Figure S6 Energy surface for the reaction of the substrate used in Shi's work

4. The chemoselectivity of $\mathbf{1 m}$

We also studied the reaction of substrate $\mathbf{1 m}$, which gave a mixture of two products $\mathbf{2 m}$ and $\mathbf{3 m}$ with a ratio of 1.3/1. Because both two chemoselectivity determination steps (through TS2-Me and TS3-Me) come from a same INT2-Me, we just discuss these two transition states here. The computed energy differences of TS2-Me and TS3-Me in $\Delta G_{\text {gas }}$ and $\Delta H_{\text {gas }}$ are only $0.7 \mathrm{kcal} / \mathrm{mol}$ and $-0.2 \mathrm{kcal} / \mathrm{mol}$ respectively, which is consistent with the observed ratio of $1.3 / 1$ for two products. However, the energy differences of them in $\Delta G_{\text {sol-Toulene }}$ and $\Delta G_{\text {sol-DCE }}$ are a little bigger, $1.6 \mathrm{kcal} / \mathrm{mol}$ and $2.3 \mathrm{kcal} / \mathrm{mol}$ respectively. It could be attributed to some errors in the solvation calculations.

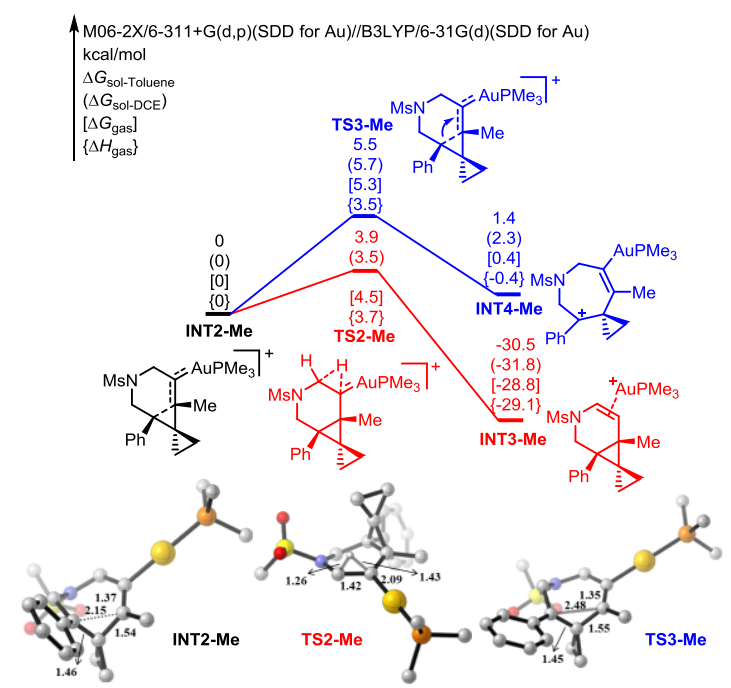

Figure S7 Chemoselectivity of $\mathbf{1 m}$

\section{Discussion of DFT functionals}

Although it had been proved that the M06-2X functional was reliable in the study of late-transition-metal-catalyzed reactions $(\mathrm{Au}, \mathrm{Pt} \text {, and } \mathrm{Ir})^{1}$. We still test several widely used functionals to validate this conclusion. The computed energy differences of TS2-Me and TS3-Me of substrate $1 \mathbf{m}$ (Figure S7) in $\Delta G_{\text {gas }}$ and $\Delta H_{\text {gas }}$ are used to evaluate different functionals. The 
smaller the difference is, the more accurate the functional is. The results of single-point energy calculations in the gas phase are given in Table S4, in which the M06-2X functional performs best.

\begin{tabular}{|c|c|c|}
\hline functional & $\Delta \Delta H_{\mathrm{gas}}{ }^{\ddagger}(\mathbf{T S 3}-\mathbf{M e}-\mathbf{T S 2}-\mathbf{M e})$ & $\Delta \Delta G_{\text {gas }}{ }^{\ddagger}($ TS3-Me - TS2-Me $)$ \\
\hline M06-2X/6-311+G(d,p) & -0.2 & 0.7 \\
\hline M06-2X/6-311G(d,p) ${ }^{2}$ & 0.1 & 1.1 \\
\hline $\mathrm{M} 06^{3} / 6-311+\mathrm{G}(\mathrm{d}, \mathrm{p})$ & -3.1 & -2.1 \\
\hline M06-L ${ }^{4} / 6-311+G(d, p)$ & -3.8 & -2.9 \\
\hline B3LYP/6-311+G(d,p) & -8.0 & -6.6 \\
\hline B3LYP-D3 $5 / 6-311+G(d, p)$ & -9.8 & -8.5 \\
\hline $\mathrm{BMK}^{6} / 6-311+\mathrm{G}(\mathrm{d}, \mathrm{p})$ & -5.0 & -3.7 \\
\hline
\end{tabular}

Table S4 Results of single-point energy calculations in the gas phase

\section{The chemoselectivity of $\mathbf{1 d}$}

As shown in Figure S8, substrate $\mathbf{1 d}$ also gives a non-classical cation intermediate $\mathbf{I N T 2}-\mathbf{C F}_{\mathbf{3}} \mathbf{P h}$. The electron-withdrawing $\mathrm{CF}_{3}$ group in the aryl ring has an influence on this non-classical cation intermediate, in which a shorter elongated C-C bond of $2.14 \AA$ is found in INT2-CF $\mathbf{I} \mathbf{P h}$ comparing to INT2-Ph. Comparing to a phenyl group in INT4-Ph, this electron-withdrawing $\mathrm{CF}_{3}$ group also has less stabilization on the cation intermediate $\mathbf{I N T 4}-\mathbf{C F}_{3} \mathbf{P h}(-0.7 \mathrm{kcal} / \mathrm{mol}$ of INT4-CF3Ph from INT2-CF3Ph vs. $-1.0 \mathrm{kcal} / \mathrm{mol}$ of INT4-Ph from INT2-Ph in $\Delta G_{\text {sol-Toulene). }}$ ). These make the expansion step become more difficult than the direct [1,2]-hydride shift $(6.2$ $\mathrm{kcal} / \mathrm{mol}$ of $\mathbf{T S 3}-\mathbf{C F}_{3} \mathbf{P h}$ vs. $3.5 \mathrm{kcal} / \mathrm{mol}$ of $\mathbf{T S 2}-\mathbf{C F}_{3} \mathbf{P h}$ in $\Delta G_{\text {sol-Toulene }}{ }^{*}$ ), which can explain that only product $\mathbf{3 d}$ was obtained experimentally.

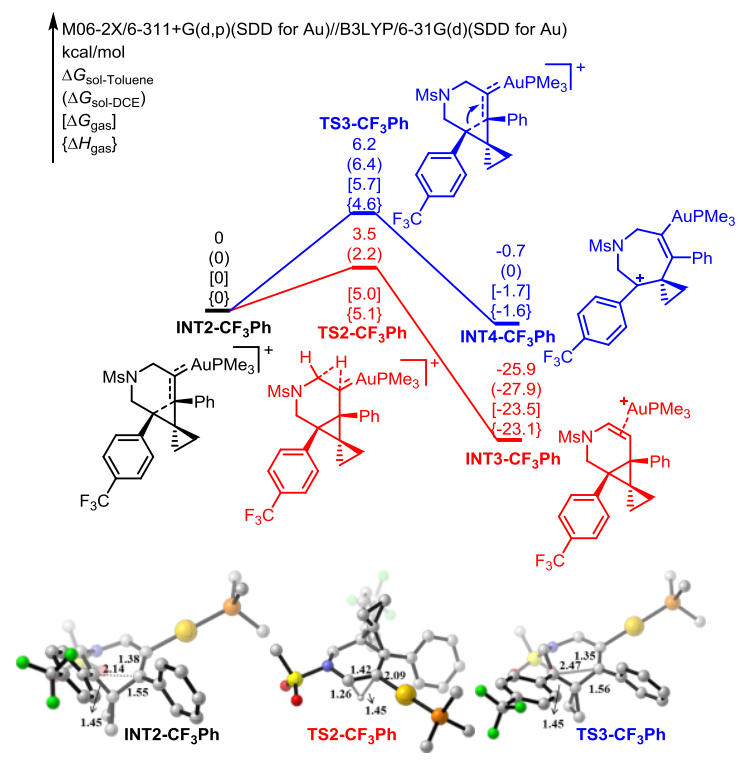

Figure S8 Chemoselectivity of $1 \mathrm{~d}$

Because of small energy differences of TS2-CF $\mathbf{P} \mathbf{P h}$ and $\mathbf{T S 3}-\mathbf{C F}_{3} \mathbf{P h}$ in $\Delta G_{\text {gas }}$ and $\Delta H_{\text {gas }}$ in Figure S8, we performed calculations using a real model of 1d. In Figure S9, it gives results consistent with the experimental observation that only product $\mathbf{3 d}$ was found. 


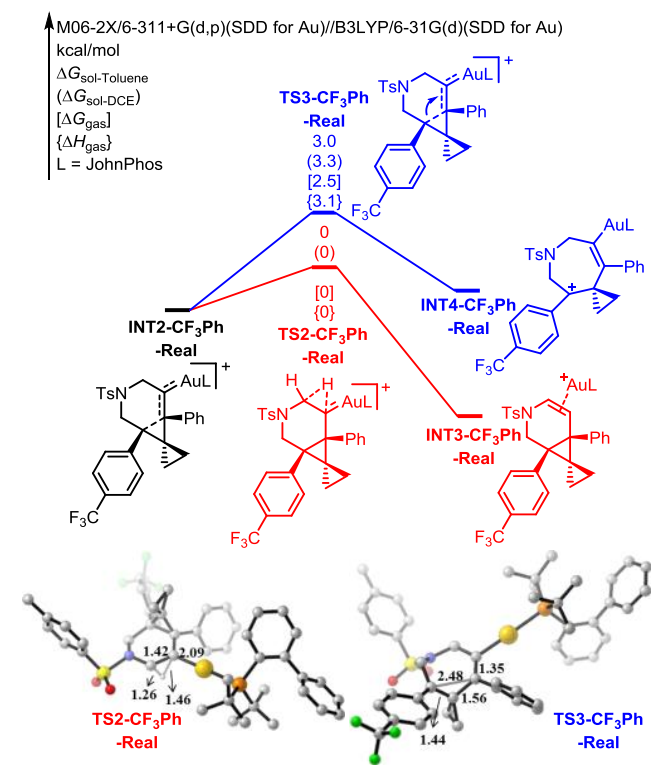

Figure S9 Chemoselectivity of $1 \mathrm{~d}$ with a real model

7. Energy data ${ }^{a}$

\begin{tabular}{|c|c|c|c|c|c|c|}
\hline & $\mathrm{E}^{b}$ & $\mathrm{H}^{c}$ & $\mathrm{G}^{d}$ & $\mathrm{E}^{e}$ & $\Delta E_{\text {sol-Toluene }}$ & $\Delta E_{\text {sol-DCE }}$ \\
\hline INT1-Ph & -2012.811279 & -2012.288116 & -2012.395264 & -2012.460546 & -0.032500 & -0.052238 \\
\hline TS1-Ph & -2012.785894 & -2012.263684 & -2012.365861 & -2012.431314 & -0.034456 & -0.055750 \\
\hline INT2-Ph & -2012.813621 & -2012.289670 & -2012.389798 & -2012.458282 & -0.034265 & -0.055397 \\
\hline TS2-Ph & -2012.785877 & -2012.266080 & -2012.365643 & -2012.443264 & -0.035964 & -0.058787 \\
\hline INT3-Ph & -2012.838817 & -2012.314256 & -2012.414706 & -2012.499084 & -0.036755 & -0.059906 \\
\hline Sub-Ph & -1416.052425 & -1415.656317 & -1415.740067 & -1415.911617 & -0.006933 & -0.013240 \\
\hline Pro1-Ph & -1416.090738 & -1415.693730 & -1415.768485 & -1415.966383 & -0.006441 & -0.012357 \\
\hline TS3-Ph & -2012.808021 & -2012.285257 & -2012.383643 & -2012.452545 & -0.034118 & -0.055211 \\
\hline INT4-Ph & -2012.813928 & -2012.289615 & -2012.389676 & -2012.461812 & -0.032631 & -0.052470 \\
\hline TS4-Ph & -2012.810217 & -2012.287076 & -2012.385808 & -2012.460152 & -0.032912 & -0.052692 \\
\hline INT5-Ph & -2012.836267 & -2012.311852 & -2012.413396 & -2012.480205 & -0.033345 & -0.053468 \\
\hline TS5-Ph & -2012.816469 & -2012.295795 & -2012.395559 & -2012.464343 & -0.035012 & -0.056448 \\
\hline INT6-Ph & -2012.878479 & -2012.352572 & -2012.451669 & -2012.531313 & -0.035661 & -0.057618 \\
\hline Pro2-Ph & -1416.132478 & -1415.734084 & -1415.807460 & -1415.997669 & -0.005474 & -0.010149 \\
\hline INT1-H & -1781.762040 & -1781.324642 & -1781.419910 & -1781.441852 & -0.033051 & -0.052646 \\
\hline TS1-H & -1781.733027 & -1781.297178 & -1781.388876 & -1781.408623 & -0.034972 & -0.056108 \\
\hline INT2-H & -1781.761588 & -1781.324071 & -1781.413119 & -1781.444546 & -0.035717 & -0.057599 \\
\hline TS2-H & -1781.741307 & -1781.306813 & -1781.396337 & -1781.428611 & -0.036084 & -0.058174 \\
\hline INT3-H & -1781.804347 & -1781.365444 & -1781.455586 & -1781.490629 & -0.037039 & -0.059729 \\
\hline Sub-H & -1185.004887 & -1184.694606 & -1184.766560 & -1184.896016 & -0.006145 & -0.011614 \\
\hline Pro1-H & -1185.049798 & -1184.738359 & -1184.803336 & -1184.951556 & -0.005679 & -0.010693 \\
\hline TS3-H & -1781.729284 & -1781.294092 & -1781.383329 & -1781.406561 & -0.035939 & -0.058024 \\
\hline INT4-H & -1781.754136 & -1781.315955 & -1781.405899 & -1781.433082 & -0.035405 & -0.056921 \\
\hline TS4-H & -1781.753607 & -1781.316348 & -1781.404018 & -1781.434237 & -0.035194 & -0.056405 \\
\hline INT5-H & -1781.793214 & -1781.354370 & -1781.444580 & -1781.465757 & -0.034567 & -0.055457 \\
\hline
\end{tabular}




\begin{tabular}{|c|c|c|c|c|c|c|}
\hline TS5-H & -1781.768954 & -1781.333682 & -1781.424221 & -1781.443402 & -0.035695 & -0.057323 \\
\hline INT6-H & -1781.832427 & -1781.392408 & -1781.482745 & -1781.508000 & -0.036984 & -0.059603 \\
\hline Pro2-H & -1185.084920 & -1184.772043 & -1184.836365 & -1184.974026 & -0.005714 & -0.010789 \\
\hline INT2-Me & -1821.080334 & -1820.612533 & -1820.705470 & -1820.746679 & -0.034664 & -0.055955 \\
\hline TS2-Me & -1821.055927 & -1820.591910 & -1820.683513 & -1820.737012 & -0.035624 & -0.057670 \\
\hline TS3-Me & -1821.073098 & -1820.606395 & -1820.696499 & -1820.740038 & -0.034283 & -0.055196 \\
\hline INT3-Me & -1821.114056 & -1820.645762 & -1820.738237 & -1820.793553 & -0.037264 & -0.060833 \\
\hline INT4-Me & -1821.077443 & -1820.609171 & -1820.700941 & -1820.747776 & -0.032967 & -0.052839 \\
\hline INT2-CF 3 Ph & -2349.840753 & -2349.308513 & -2349.418660 & -2349.502938 & -0.035549 & -0.057781 \\
\hline $\mathrm{TS2}_{2}-\mathrm{CF}_{3} \mathrm{Ph}$ & -2349.816170 & -2349.287974 & -2349.398311 & -2349.490694 & -0.037904 & -0.062215 \\
\hline $\mathrm{TS3}^{-\mathrm{CF}_{3} \mathrm{Ph}}$ & -2349.833534 & -2349.302717 & -2349.411085 & -2349.494236 & -0.034767 & -0.056653 \\
\hline INT3-CF ${ }_{3} P h$ & -2349.866249 & -2349.333552 & -2349.444364 & -2349.540098 & -0.039307 & -0.064806 \\
\hline INT4-CF 3 Ph & -2349.840764 & -2349.308300 & -2349.418711 & -2349.505698 & -0.033945 & -0.054958 \\
\hline TS2-CF 3 Ph-Real & -3239.488987 & -3238.553705 & -3238.710447 & -3239.036451 & -0.031570 & -0.052392 \\
\hline TS3-CF 3 Ph-Real & -3239.500293 & -3238.562770 & -3238.720578 & -3239.033691 & -0.030801 & -0.051038 \\
\hline
\end{tabular}

$a$ in a.u.

${ }^{b}$ single-point energies at the level of B3LYP/6-31G(d) (SDD for Au)

${ }^{c}$ enthalpies at the level of B3LYP/6-31G(d) (SDD for Au)

${ }^{d}$ Gibbs free energies at the level of B3LYP/6-31G(d) (SDD for Au)

${ }^{e}$ single-point energies at the level of M06-2X/6-311+G(d,p) (SDD for Au)

The single-point energies for Figure S5

\begin{tabular}{|c|c|c|c|c|c|c|c|c|c|}
\hline 2.13 & -2012.788649 & -2012.788881 & -2012.788997 & -2012.788975 & -2012.788793 & -2012.788425 & -2012.787843 & -2012.787012 & -2012.785895 \\
\hline 2.07 & -2012.790841 & -2012.791065 & -2012.7911701 & -2012.791136 & -2012.790941 & -2012.790558 & -2012.789958 & -2012.789110 & -2012.787977 \\
\hline 2.01 & -2012.793106 & -2012.793313 & -2012.793400 & -2012.793346 & -2012.793131 & -2012.792727 & -2012.792107 & -2012.791239 & -2012.790095 \\
\hline 1.95 & -2012.795486 & -2012.795662 & -2012.795716 & -2012.795629 & -2012.795379 & -2012.794940 & -2012.794281 & -2012.793390 & -2012.792220 \\
\hline 1.89 & -2012.798161 & -2012.798273 & -2012.798265 & -2012.798119 & -2012.797812 & -2012.797319 & -2012.796612 & -2012.795660 & -2012.794426 \\
\hline 1.83 & -2012.801152 & -2012.801211 & -2012.801149 & -2012.800946 & -2012.800582 & -2012.800033 & -2012.799271 & -2012.798265 & -2012.796982 \\
\hline 1.77 & -2012.804389 & -2012.804399 & -2012.804286 & -2012.804032 & -2012.803616 & -2012.803015 & -2012.802203 & -2012.801148 & -2012.799818 \\
\hline 1.71 & -2012.807704 & -2012.807675 & -2012.807524 & -2012.807233 & -2012.806782 & -2012.806149 & -2012.805307 & -2012.804227 & -2012.802876 \\
\hline 1.65 & -2012.810768 & -2012.810718 & -2012.810547 & -2012.810240 & -2012.809775 & -2012.809131 & -2012.808282 & -2012.807198 & -2012.805850 \\
\hline 1.59 & -2012.813105 & -2012.813044 & -2012.812864 & -2012.812549 & -2012.812080 & -2012.811436 & -2012.810595 & -2012.809531 & -2012.808216 \\
\hline 1.53 & -2012.813913 & -2012.813861 & -2012.813693 & -2012.813393 & -2012.812945 & -2012.812328 & -2012.811522 & -2012.810505 & -2012.809256 \\
\hline & 1.68 & 1.66 & 1.64 & 1.62 & 1.60 & 1.58 & 1.56 & 1.54 & 1.52 \\
\hline
\end{tabular}

Energy surface calculated at the level of B3LYP/6-31G(d) (SDD for Au) (a.u.)

The first column in red $\mathrm{C} 2-\mathrm{C} 3$ bond distances

The last row in blue is $\mathrm{C} 2-\mathrm{C} 4$ bond distance

Others in black are single-point energies

8. Cartesian coordinates of all stationary points

\section{INT1-Ph}

C $\quad-0.87851800 \quad-1.01572200 \quad-1.50517400$

$\begin{array}{lrrr}\text { C } & -0.28302300 & 0.27618700 & -1.03007000 \\ \text { C } & -0.21267200 & 1.51307200 & -0.93636800 \\ \text { C } & -1.79681300 & -1.81615700 & 0.68981700\end{array}$




\begin{tabular}{|c|c|c|c|}
\hline $\mathrm{C}$ & -3.23055700 & -1.43277500 & 0.33036400 \\
\hline $\mathrm{C}$ & -3.99607100 & -2.31689000 & -0.31275400 \\
\hline $\mathrm{C}$ & -5.30594700 & -2.68719600 & -0.86036300 \\
\hline $\mathrm{C}$ & -4.12449400 & -3.66933400 & -0.87361000 \\
\hline $\mathrm{C}$ & -0.23903600 & 2. 93530300 & -0.82471400 \\
\hline $\mathrm{C}$ & 0.56011100 & 3. 73277300 & -1.67167400 \\
\hline $\mathrm{C}$ & 0.49420100 & 5. 11848100 & -1.57732800 \\
\hline $\mathrm{C}$ & -0.36254900 & 5. 71708200 & -0.64716700 \\
\hline $\mathrm{C}$ & -1.15875100 & 4. 93049000 & 0.19120500 \\
\hline $\mathrm{C}$ & -1.10276100 & 3. 54236700 & 0.11140600 \\
\hline $\mathrm{N}$ & -0.98324400 & -2.09504100 & -0.52389600 \\
\hline S & 0.42291000 & -3.01517500 & -0.25870100 \\
\hline 0 & 0.93978100 & -2.78649500 & 1. 10204900 \\
\hline 0 & 1. 31144000 & -2.78971000 & -1.40976400 \\
\hline $\mathrm{C}$ & -3.74135000 & -0.09547200 & 0.73933800 \\
\hline $\mathrm{C}$ & -4.65179300 & 0.60670800 & -0.07148200 \\
\hline $\mathrm{C}$ & -5.16260300 & 1. 84164000 & 0.32398200 \\
\hline $\mathrm{C}$ & -4.77782600 & 2. 40636300 & 1. 54276400 \\
\hline $\mathrm{C}$ & -3.87476900 & 1. 72340200 & 2. 36044700 \\
\hline $\mathrm{C}$ & -3.35808700 & 0.48908800 & 1. 96084800 \\
\hline $\mathrm{H}$ & -1.89752300 & -0.79751000 & -1.84011400 \\
\hline $\mathrm{H}$ & -0.30439300 & -1.37708200 & -2.36002100 \\
\hline H & -1.78821900 & -2.72245900 & 1. 30135500 \\
\hline H & -1.31841600 & -1.02745800 & 1. 27992400 \\
\hline $\mathrm{H}$ & -6.12566800 & -2.87479300 & -0.16799400 \\
\hline $\mathrm{H}$ & -5.62508900 & -2.26873800 & -1.81385300 \\
\hline $\mathrm{H}$ & -3.66223300 & -3.89498300 & -1.83405600 \\
\hline $\mathrm{H}$ & -4.16370900 & -4.51999500 & -0.19338600 \\
\hline $\mathrm{H}$ & 1. 21584500 & 3. 25679400 & -2.39439000 \\
\hline $\mathrm{H}$ & 1. 10610800 & 5. 73379800 & -2.22960900 \\
\hline $\mathrm{H}$ & -0.41152200 & 6. 79985200 & -0.57833400 \\
\hline $\mathrm{H}$ & -1.82651100 & 5. 40057700 & 0.90651600 \\
\hline $\mathrm{H}$ & -1.72477700 & 2. 92395200 & 0.75093100 \\
\hline $\mathrm{H}$ & -4.95383400 & 0.17946700 & -1.02247100 \\
\hline $\mathrm{H}$ & -5.86326800 & 2. 36428000 & -0.32126100 \\
\hline $\mathrm{H}$ & -5.18487200 & 3. 36374100 & 1. 85546700 \\
\hline $\mathrm{H}$ & -3.58365300 & 2. 14102600 & 3. 32062600 \\
\hline $\mathrm{H}$ & -2.68394300 & -0.03833200 & 2. 63023600 \\
\hline $\mathrm{C}$ & -0.19949600 & -4.70157500 & -0.33896700 \\
\hline $\mathrm{H}$ & -0.58513700 & -4.87803300 & -1.34373700 \\
\hline $\mathrm{H}$ & 0.64606500 & -5.35933100 & -0.12421500 \\
\hline $\mathrm{H}$ & -0.98082200 & -4.83694000 & 0.41084300 \\
\hline $\mathrm{Au}$ & 1. 74740200 & 0.32674700 & -0.12857900 \\
\hline & 3.81476000 & -0.28875700 & 0.74465000 \\
\hline
\end{tabular}

$\begin{array}{lrrr}\text { C } & 3.66303500 & -1.11192600 & 2.37604900 \\ \text { H } & 2.96886600 & -1.95195900 & 2.28471800 \\ \text { H } & 4.64194100 & -1.46867400 & 2.71419300 \\ \text { H } & 3.26374900 & -0.40634300 & 3.11070200 \\ \text { C } & 4.64301500 & -1.49897600 & -0.35781900 \\ \text { H } & 4.90203500 & -1.01680900 & -1.30505400 \\ \text { H } & 5.55383700 & -1.88542600 & 0.11186500 \\ \text { H } & 3.95375000 & -2.32131500 & -0.56817200 \\ \text { C } & 5.01395900 & 1.08360600 & 0.98019100 \\ \text { H } & 5.95685100 & 0.69670800 & 1.38169900 \\ \text { H } & 5.20728700 & 1.57783600 & 0.02377000 \\ \text { H } & 4.60422500 & 1.82101300 & 1.67650100\end{array}$

\section{TS1-Ph}

\begin{tabular}{|c|c|c|c|}
\hline $\mathrm{C}$ & 2. 64189700 & 1. 34222500 & -0.90142000 \\
\hline $\mathrm{C}$ & -0.17342200 & 0.59027100 & -0.00711400 \\
\hline $\mathrm{C}$ & 2. 55795900 & 0.00965900 & 1. 20522600 \\
\hline $\mathrm{C}$ & 2. 44050500 & 0.82008100 & 2. 43314300 \\
\hline $\mathrm{C}$ & 2.94403100 & -0.62206500 & 2. 48016700 \\
\hline $\mathrm{N}$ & 1. 70262800 & 2. 23766800 & -0.24926700 \\
\hline S & 2. 22911800 & 3. 67030900 & 0.46234900 \\
\hline 0 & 1. 23276300 & 4. 02441000 & 1. 47396100 \\
\hline 0 & 3. 64101300 & 3. 46586000 & 0.79067100 \\
\hline $\mathrm{H}$ & 2. 25381400 & 1. 09915900 & -1.89938400 \\
\hline $\mathrm{H}$ & 3. 59751700 & 1. 85918600 & -1.04107400 \\
\hline $\mathrm{H}$ & 3. 14822200 & 1. 63533800 & 2. 56173100 \\
\hline $\mathrm{H}$ & 1. 45606000 & 1. 01955400 & 2. 84878300 \\
\hline $\mathrm{H}$ & 2. 29061200 & -1.36795100 & 2. 92857000 \\
\hline $\mathrm{H}$ & 4. 00439600 & -0.80696900 & 2. 64798800 \\
\hline $\mathrm{C}$ & 2. 15296700 & 4. 92058200 & -0.83826400 \\
\hline $\mathrm{H}$ & 2. 48931800 & 5. 86200300 & -0.39698600 \\
\hline $\mathrm{H}$ & 2. 81842800 & 4. 63000500 & -1.65364300 \\
\hline $\mathrm{H}$ & 1. 12190300 & 5. 01740100 & -1.18497600 \\
\hline $\mathrm{C}$ & 2. 91037200 & 0.05981600 & -0.11534600 \\
\hline $\mathrm{C}$ & 0.41873500 & -0.46349700 & 0.44639600 \\
\hline $\mathrm{C}$ & 0.32826800 & -1.84815600 & 0.81858700 \\
\hline $\mathrm{C}$ & -0.06038100 & -2.20647100 & 2. 12965500 \\
\hline $\mathrm{C}$ & 0.58174500 & -2.86439000 & -0.12805800 \\
\hline $\mathrm{C}$ & -0.20700800 & -3.54668400 & 2. 47415400 \\
\hline $\mathrm{H}$ & -0.26759100 & -1.42509600 & 2. 85479900 \\
\hline $\mathrm{C}$ & 0.43537100 & -4.20284200 & 0.22876500 \\
\hline $\mathrm{H}$ & 0.87624100 & -2.59215400 & -1.13564400 \\
\hline $\mathrm{C}$ & 0.04359900 & -4.54556100 & 1. 52620100 \\
\hline H & -0.51760200 & -3.81513500 & 3. 47945900 \\
\hline
\end{tabular}




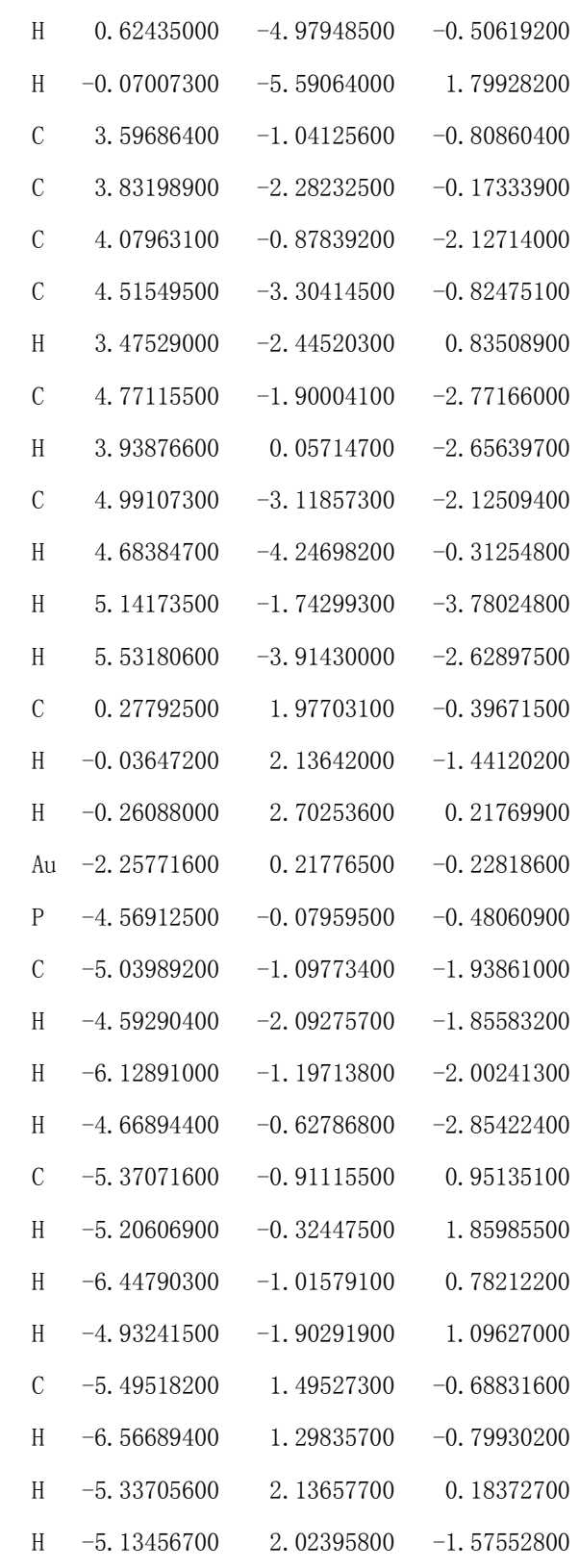

\section{INT2-Ph}

$\begin{array}{lrrr}\text { C } & -2.81941200 & -0.97011400 & -0.89346200 \\ \text { C } & -0.01632000 & -0.61850000 & 0.14472000 \\ \text { C } & -2.17409000 & 0.26902500 & 1.21909200 \\ \text { C } & -2.64823200 & -0.62596100 & 2.33424000 \\ \text { C } & -2.76867700 & 0.86978500 & 2.46752200 \\ \text { N } & -2.07444700 & -2.03790700 & -0.26975400 \\ \text { S } & -2.85440000 & -3.41855200 & 0.29499400 \\ \text { O } & -2.04057100 & -3.94116500 & 1.39169800 \\ \text { O } & -4.25930700 & -3.03781700 & 0.47419100 \\ \text { H } & -2.51160600 & -0.84242100 & -1.94060300 \\ \text { H } & -3.88217900 & -1.24804000 & -0.89119700 \\ \text { H } & -3.55365400 & -1.20378100 & 2.17684800\end{array}$

\begin{tabular}{|c|c|c|c|}
\hline & -1.88354900 & -1.13856900 & 2. 90995700 \\
\hline & -2.11546700 & 1. 39100400 & 3. 15836200 \\
\hline & -3.76152400 & 1. 30432700 & 2. 38528900 \\
\hline & -2.78097300 & -4.61435500 & -1.05412900 \\
\hline & -3.26630800 & -5.52689400 & -0.69929600 \\
\hline & -3.31669100 & -4.21414500 & -1.91720600 \\
\hline & -1.73546200 & -4.81728400 & -1.29587700 \\
\hline & -2.71142200 & 0.34263100 & -0.13364400 \\
\hline & -0.70414900 & 0.40732100 & 0.74469800 \\
\hline & -0.05954300 & 1. 74452100 & 0.92908600 \\
\hline & 0.27034800 & 2. 21214900 & 2. 21390900 \\
\hline & 0.26743500 & 2. 54660700 & -0.17891200 \\
\hline & 0.91201500 & 3. 43980900 & 2. 38323000 \\
\hline & 0.06594500 & 1. 59350500 & 3. 08271700 \\
\hline & 0.89648300 & 3. 78099100 & -0.00818900 \\
\hline & 0.02464200 & 2. 19583800 & -1.17789600 \\
\hline & 1. 22007100 & 4. 23130300 & 1. 27363100 \\
\hline & 1. 17377400 & 3. 77606500 & 3. 38245000 \\
\hline & 1. 13334200 & 4. 38952800 & -0.87664400 \\
\hline & 1. 71053700 & 5. 19113900 & 1. 40816300 \\
\hline$C$ & -3.15927000 & 1. 56636500 & -0.75506300 \\
\hline $\mathrm{C}$ & -3.08806500 & 2. 80971700 & -0.07094800 \\
\hline & -3.73451000 & 1. 55513100 & -2.05564200 \\
\hline C & -3.56635700 & 3. 97317100 & -0.65842700 \\
\hline H & -2.64756700 & 2. 85448000 & 0.91523800 \\
\hline $\mathrm{C}$ & -4.22695600 & 2. 71852100 & -2.62688300 \\
\hline H & -3.81671000 & 0.63025600 & -2.61400600 \\
\hline 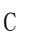 & -4.14158900 & 3. 93196000 & -1.93217200 \\
\hline 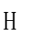 & -3.49877000 & 4. 91394800 & -0.12134500 \\
\hline I & -4.67712500 & 2. 68665400 & -3.61403300 \\
\hline t & -4.52561300 & 4. 84189600 & -2.38409400 \\
\hline C & -0.61401400 & -1.94248400 & -0.26532700 \\
\hline & -0.21771200 & -2.18366500 & -1.26612800 \\
\hline 4 & -0.23092900 & -2.71335200 & 0.41426700 \\
\hline $\mathrm{Au}$ & 2. 03441700 & -0.48036300 & -0.17670600 \\
\hline & 4. 37586200 & -0.37808200 & -0.48946800 \\
\hline C & 4. 94341800 & 1. 04501400 & -1.51094100 \\
\hline 1 & 4. 62801300 & 1.98254800 & -1.04356100 \\
\hline H & 6. 03418700 & 1.04239900 & -1.61018200 \\
\hline & 4. 49326800 & 0.98859000 & -2.50654400 \\
\hline 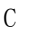 & 5. 33003800 & -0.22342200 & 1. 07741500 \\
\hline 1 & 5. 12443700 & -1.08306700 & 1. 72215700 \\
\hline & 6. 40536500 & -0.17635200 & 0.87453500 \\
\hline & 5. 02428500 & 0.68358500 & 1. 60717600 \\
\hline
\end{tabular}




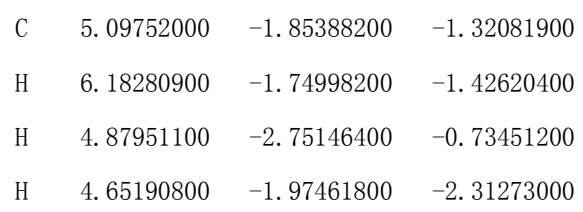

\section{TS2-Ph}

\begin{tabular}{|c|c|c|c|}
\hline C & 2. 83550500 & 1. 18819500 & -0.22322300 \\
\hline $\mathrm{C}$ & -0.05714800 & 0.57556500 & -0.06991700 \\
\hline $\mathrm{C}$ & 1. 56630700 & -0.20089300 & 1. 57602800 \\
\hline $\mathrm{C}$ & 1. 25665600 & 0.70916600 & 2. 71954800 \\
\hline $\mathrm{C}$ & 1. 53754600 & -0.76312800 & 2. 95603400 \\
\hline $\mathrm{N}$ & 1. 79736700 & 2. 23006100 & -0.10878100 \\
\hline S & 2. 24878100 & 3. 82951200 & -0.66240200 \\
\hline 0 & 3.23776700 & 3. 66411400 & -1.72223700 \\
\hline 0 & 0.98696600 & 4. 53589100 & -0.87736900 \\
\hline $\mathrm{H}$ & 3. 10631100 & 1. 04801600 & -1.27621000 \\
\hline $\mathrm{H}$ & 3.73437300 & 1.53696500 & 0.29584900 \\
\hline $\mathrm{H}$ & 2. 02000900 & 1. 42723000 & 3. 00928300 \\
\hline $\mathrm{H}$ & 0.23685700 & 1. 05936300 & 2. 85948200 \\
\hline $\mathrm{H}$ & 0.69505800 & -1.39113000 & 3. 23227800 \\
\hline $\mathrm{H}$ & 2. 47673800 & -1.04812700 & 3. 42208000 \\
\hline $\mathrm{C}$ & 3. 05665000 & 4. 50174900 & 0.79717000 \\
\hline H & 3. 40026700 & 5.50167200 & 0.51995900 \\
\hline $\mathrm{H}$ & 2. 32947800 & 4. 55589100 & 1. 60839000 \\
\hline H & 3. 91108600 & 3. 87646700 & 1. 06243900 \\
\hline $\mathrm{C}$ & 2. 40330500 & -0.15941900 & 0.36369400 \\
\hline $\mathrm{C}$ & 0.87690500 & -0.52419900 & 0.24513100 \\
\hline C & 0.45809800 & -1.91884600 & -0.14361900 \\
\hline $\mathrm{C}$ & 0.71225800 & -3.04160900 & 0.65645600 \\
\hline C & -0.17458000 & -2.11686700 & -1.38364200 \\
\hline $\mathrm{C}$ & 0.32222800 & -4.31643000 & 0.24169600 \\
\hline H & 1. 23366600 & -2.93060900 & 1. 59890800 \\
\hline $\mathrm{C}$ & -0.56186000 & -3.39150100 & -1.80055900 \\
\hline $\mathrm{H}$ & -0.35205000 & -1.27086800 & -2.04292500 \\
\hline C & -0.32053500 & -4.49748800 & -0.98386800 \\
\hline H & 0.52987400 & -5.17133200 & 0.87886800 \\
\hline H & -1.03953900 & -3.51865400 & -2.76816700 \\
\hline H & -0.61726100 & -5.49147100 & -1.30556300 \\
\hline $\mathrm{C}$ & 3. 45071200 & -1.21006000 & 0.09022300 \\
\hline $\mathrm{C}$ & 4. 40581300 & -1.51922600 & 1. 06804200 \\
\hline $\mathrm{C}$ & 3.55571700 & -1.80999200 & -1.17329000 \\
\hline $\mathrm{C}$ & 5. 43385600 & -2.42392100 & 0.79641500 \\
\hline H & 4. 35588500 & -1.04383900 & 2. 04347100 \\
\hline$C$ & 4. 58071800 & -2.71534400 & -1.44464000 \\
\hline
\end{tabular}

$$
\begin{array}{lrrr}
\mathrm{H} & 2.82536300 & -1.57949400 & -1.94384100 \\
\mathrm{C} & 5.52132400 & -3.02572500 & -0.45959600 \\
\mathrm{H} & 6.16620500 & -2.65450100 & 1.56473000 \\
\mathrm{H} & 4.64513900 & -3.17770900 & -2.42528500 \\
\mathrm{H} & 6.32021700 & -3.73017600 & -0.67188300 \\
\mathrm{C} & 0.46492200 & 1.87993800 & -0.28313500 \\
\mathrm{H} & 0.16825000 & 1.24816200 & -1.33642300 \\
\mathrm{H} & -0.21093000 & 2.73018500 & -0.32721900 \\
\mathrm{Au} & -2.12815500 & 0.27755600 & -0.03167200 \\
\mathrm{P} & -4.45382600 & -0.05869600 & 0.09988300 \\
\mathrm{C} & -5.16257500 & -0.89247800 & -1.37886500 \\
\mathrm{H} & -4.69165700 & -1.87079200 & -1.51227600 \\
\mathrm{H} & -6.24343600 & -1.02713800 & -1.26373400 \\
\mathrm{H} & -4.97055200 & -0.29115900 & -2.27240000 \\
\mathrm{C} & -4.96377800 & -1.11003700 & 1.52060500 \\
\mathrm{H} & -4.65494400 & -0.64117100 & 2.45957600 \\
\mathrm{H} & -6.05077600 & -1.24410100 & 1.52859900 \\
\mathrm{H} & -4.48178000 & -2.08946900 & 1.44844500 \\
\mathrm{C} & -5.43049100 & 1.48851400 & 0.28252900 \\
\mathrm{H} & -6.50066200 & 1.26272000 & 0.33945400 \\
\mathrm{H} & -5.12633800 & 2.01437100 & 1.19228900 \\
\mathrm{H} & -5.24848700 & 2.14576800 & -0.57291100 \\
\hline
\end{array}
$$

\begin{tabular}{|c|c|c|c|}
\hline $\mathrm{C}$ & 0.12570500 & -0.42264300 & -0.94041800 \\
\hline $\mathrm{C}$ & 1. 13951600 & -0.91213900 & 1. 46681300 \\
\hline C & 0.05022300 & -1.10937700 & 2. 46587000 \\
\hline $\mathrm{C}$ & 1. 35630700 & -1.88221100 & 2. 57488500 \\
\hline N & 0.45500100 & 1. 91976000 & -0.43488100 \\
\hline S & -0.24412000 & 3. 53050700 & -0.61991100 \\
\hline 0 & -1.07243100 & 3. 46094700 & -1.82066300 \\
\hline 0 & -0.79417600 & 3. 88290200 & 0.68474100 \\
\hline H & -0.14441800 & -0.33140600 & 3. 20188500 \\
\hline $\mathrm{H}$ & -0.83543200 & -1.66235400 & 2. 15658300 \\
\hline $\mathrm{H}$ & 1. 34737000 & -2.94644500 & 2. 35753400 \\
\hline $\mathrm{H}$ & 2. 02965900 & -1.59909300 & 3. 37938500 \\
\hline $\mathrm{C}$ & 1. 20359700 & 4. 54147800 & -0.95579900 \\
\hline H & 0.83660900 & 5. 56617200 & -1.05908100 \\
\hline $\mathrm{H}$ & 1. 89792800 & 4. 47634800 & -0.11635200 \\
\hline $\mathrm{H}$ & 1. 66048900 & 4. 20171600 & -1.88651800 \\
\hline $\mathrm{C}$ & 1. 83453700 & 0.28224300 & 0.88833600 \\
\hline $\mathrm{C}$ & 1. 25284400 & -0.86578300 & -0.01965200 \\
\hline $\mathrm{C}$ & 2. 16996100 & -1.83125600 & -0.73512700 \\
\hline $\mathrm{C}$ & 2. 00480900 & -3.21219300 & -0.55796500 \\
\hline
\end{tabular}

\section{INT3-Ph}




\begin{tabular}{|c|c|c|c|}
\hline C & 3. 11967600 & -1.37905900 & -1.66277900 \\
\hline $\mathrm{C}$ & 2. 78263100 & -4.12151000 & -1.27743700 \\
\hline $\mathrm{H}$ & 1. 25945900 & -3.58141800 & 0.14103500 \\
\hline $\mathrm{C}$ & 3. 90018800 & -2.28639600 & -2.37916300 \\
\hline H & 3. 26224400 & -0.31288100 & -1.81241000 \\
\hline $\mathrm{C}$ & 3. 73468900 & -3.66003100 & -2.18771800 \\
\hline $\mathrm{H}$ & 2. 64374100 & -5.18830000 & -1.12636400 \\
\hline $\mathrm{H}$ & 4. 63915800 & -1.91997400 & -3.08604700 \\
\hline $\mathrm{H}$ & 4. 34277500 & -4.36583500 & -2.74608000 \\
\hline $\mathrm{C}$ & 3. 32562100 & 0.50143600 & 0.97505900 \\
\hline C & 4. 18418600 & -0.41972500 & 1. 59282500 \\
\hline $\mathrm{C}$ & 3.90093100 & 1. 64556700 & 0.39598400 \\
\hline $\mathrm{C}$ & 5. 55968100 & -0.19157600 & 1. 65197100 \\
\hline $\mathrm{H}$ & 3.78494600 & -1.32946800 & 2. 02210100 \\
\hline $\mathrm{C}$ & 5.27555700 & 1. 87515600 & 0.45201000 \\
\hline $\mathrm{H}$ & 3. 27496300 & 2. 36220400 & -0.12912000 \\
\hline $\mathrm{C}$ & 6. 11286100 & 0.95814100 & 1. 08832400 \\
\hline $\mathrm{H}$ & 6. 19959700 & -0.91969800 & 2. 14224900 \\
\hline H & 5. 69110000 & 2. 76895400 & -0.00511100 \\
\hline $\mathrm{H}$ & 7. 18320300 & 1. 13457400 & 1. 13759200 \\
\hline $\mathrm{C}$ & -0.04558800 & 0.94099400 & -1.21906600 \\
\hline $\mathrm{H}$ & -0.02571300 & -1.06766700 & -1.80714300 \\
\hline $\mathrm{H}$ & -0.56629900 & 1. 27640600 & -2.11119000 \\
\hline $\mathrm{C}$ & 0.97452400 & 1. 55336400 & 0.90803700 \\
\hline $\mathrm{H}$ & 0.10629000 & 1. 41869100 & 1. 56184200 \\
\hline $\mathrm{H}$ & 1. 54867400 & 2. 3953 & 1. 29401300 \\
\hline $\mathrm{Au}$ & -2.01185000 & -0.47894200 & -0.23037100 \\
\hline P & -4.26260200 & -0.69976100 & 0.38779200 \\
\hline $\mathrm{C}$ & -5.06707500 & -2.19479300 & -0.31643800 \\
\hline $\mathrm{H}$ & -4.55059700 & -3.09502000 & 0.02938200 \\
\hline $\mathrm{H}$ & -6.11733000 & -2.24415300 & -0.00956900 \\
\hline $\mathrm{H}$ & -5.01467800 & -2.16402800 & -1.40868800 \\
\hline $\mathrm{C}$ & -4.53850500 & -0.81162500 & 2. 20164900 \\
\hline $\mathrm{H}$ & -4.14378500 & 0.08221100 & 2. 69352900 \\
\hline $\mathrm{H}$ & -5.60883500 & -0.89590700 & 2. 41846600 \\
\hline $\mathrm{H}$ & -4.02261900 & -1.68762600 & 2. 60564600 \\
\hline $\mathrm{C}$ & -5.29666300 & 0.71399300 & -0.16879900 \\
\hline $\mathrm{H}$ & -6.33727200 & 0.56885600 & 0.14121600 \\
\hline $\mathrm{H}$ & -4.91882700 & 1. 64481800 & 0.26393300 \\
\hline $\mathrm{H}$ & -5.25477400 & 0.79826200 & -1.25857200 \\
\hline
\end{tabular}

\section{Sub-Ph}

C $\quad 0.16804600 \quad-1.41184000 \quad-1.37964400$

C $\quad 1.43429000 \quad-0.72607400 \quad-1.09720300$

\begin{tabular}{|c|c|c|c|}
\hline C & 2. 47731300 & -0.15740500 & -0.85237200 \\
\hline $\mathrm{C}$ & -1.10124900 & -0.43178900 & 0.52512000 \\
\hline $\mathrm{C}$ & -2.22378100 & 0.34320000 & -0.16064300 \\
\hline C & -3.01539100 & -0.22086100 & -1.07492600 \\
\hline $\mathrm{C}$ & -4.18926400 & -0.10097300 & -1.95413000 \\
\hline C & -3.40663500 & -1.41524100 & -1.83289300 \\
\hline $\mathrm{C}$ & 3. 71527900 & 0.49148600 & -0.55309400 \\
\hline $\mathrm{C}$ & 4. 07691500 & 1. 68556700 & -1.20610700 \\
\hline $\mathrm{C}$ & 5.28595500 & 2. 31058700 & -0.90969900 \\
\hline $\mathrm{C}$ & 6. 14871200 & 1. 75954100 & 0.04127800 \\
\hline C & 5. 79703700 & 0.57611700 & 0.69591600 \\
\hline $\mathrm{C}$ & 4. 59168200 & -0.05759100 & 0.40327700 \\
\hline $\mathrm{N}$ & -0.69365400 & -1.64492800 & -0.19875000 \\
\hline S & -0.26023300 & -2.97582700 & 0.77347500 \\
\hline 0 & 0.26377900 & -2.53652000 & 2. 07228300 \\
\hline 0 & 0.51022100 & -3.88722300 & -0.07779500 \\
\hline $\mathrm{C}$ & -2.41404500 & 1. 75074300 & 0.28476500 \\
\hline $\mathrm{C}$ & -3.03169900 & 2. 70372300 & -0.54688900 \\
\hline $\mathrm{C}$ & -3.22174200 & 4. 01589800 & -0.12156200 \\
\hline $\mathrm{C}$ & -2.79757100 & 4. 41585100 & 1. 14839600 \\
\hline $\mathrm{C}$ & -2.17712500 & 3. 48738300 & 1. 98382600 \\
\hline $\mathrm{C}$ & -1.98398600 & 2. 17300500 & 1. 55657500 \\
\hline $\mathrm{H}$ & -0.43211900 & -0.81715300 & -2.07751100 \\
\hline $\mathrm{H}$ & 0.35658700 & -2.38181200 & -1.84600700 \\
\hline $\mathrm{H}$ & -1.41365900 & -0.72304800 & 1.53358100 \\
\hline H & -0.23087700 & 0.22396300 & 0.66347800 \\
\hline H & -5.17972600 & -0.03990600 & -1.50317000 \\
\hline $\mathrm{H}$ & -4.10217000 & 0.45058700 & -2.89008200 \\
\hline $\mathrm{H}$ & -2.80591800 & -1.73184900 & -2.68535000 \\
\hline H & -3.87541600 & -2.24354900 & -1.30239200 \\
\hline $\mathrm{H}$ & 3. 40238700 & 2. 11183900 & -1.94243900 \\
\hline $\mathrm{H}$ & 5.55505700 & 3. 23100700 & -1.42090200 \\
\hline H & 7. 09060600 & 2. 25016400 & 0.27130400 \\
\hline $\mathrm{H}$ & 6. 46472700 & 0.14414000 & 1. 43642400 \\
\hline $\mathrm{H}$ & 4. 31297700 & -0.97744200 & 0.90783300 \\
\hline H & -3.34962100 & 2. 41235200 & -1.54167700 \\
\hline $\mathrm{H}$ & -3.69603000 & 4. 73191900 & -0.78788300 \\
\hline $\mathrm{H}$ & -2.94441400 & 5. 44041600 & 1. 47908800 \\
\hline H & -1.84000900 & 3. 78282600 & 2. 97387400 \\
\hline $\mathrm{H}$ & -1.50364400 & 1. 47238100 & 2. 23253400 \\
\hline $\mathrm{C}$ & -1.87462900 & -3.72397200 & 1. 07417400 \\
\hline $\mathrm{H}$ & -2.29028700 & -4.04927600 & 0.11990100 \\
\hline $\mathrm{H}$ & -1.70883900 & -4.57657500 & 1. 73702200 \\
\hline $\mathrm{H}$ & -2.53098700 & -2.99743800 & 1. 55712200 \\
\hline
\end{tabular}




\section{Pro1-Ph}

C -1.36632200

C $\quad-0.51098900$

0. 74377000

$-0.30709000$

C $\quad 0.12955200$

$-1.94991700$

$-0.54432400$

C $\quad-0.76417600$

$-0.47938700$

1. 42616100

C $\quad 0.65588000$

$-0.88382900$

2. 54053700

N $\quad-2.28675800$

$-0.41319100$

2. 81911500

S $\quad-3.94510700$

$-0.39561100$

$-0.18918900$

$0 \quad-4.60156700$

$-0.09144800$

$-0.17419200$

0 -4. 15574200

$-1.37447000$

0. 07237300

H -1.25424800

1. 07015500

0. 68937200

H -1.82032800

1. 00538500

$-1.37088600$

H $\quad-1.58679000$

1. 58851500

0. 21562000

H $\quad-0.98706100$

$-0.22401200$

2. 80533600

H $\quad 1.38942700$

$-1.94260900$

2. 65260600

H

0. 79117400

-1. 14883200

3. 14061100

C $\quad-4.35469300$

0. 57033800

3. 26422700

H $\quad-5.42682200$

0. 41064700

$-1.86358900$

H $\quad-3.79322900$

0. 61971400

$-1.88519300$

H $\quad-4.11591700$

1. 31160100

$-2.11990400$

C $\quad 0.02510400$

$-0.40733700$

-2. 54655500

C $\quad 0.50032200$

0. 45207700

0. 26829400

$-1.03997700$

0. 08005600

C $\quad 1.92021300$

$-1.37882200$

-0. 29170200

C $\quad 2.69480700$

$-2.21626000$

0.52239900

C 2.45953900

$-0.94590700$

$-1.51161500$

C $\quad 3.98182000$

$-2.59869400$

0. 13666200

H 2.28393100

$-2.57924300$

1. 46024700

C $\quad 3.74426100$

$-1.32531000$

$-1.89964400$

H 1.86803300

$-0.29962600$

$-2.15423100$

C 4.51115400

$-2.15203000$

$-1.07491300$

H $\quad 4.56771500$

$-3.24826600$

0.78189000

H 4. 14780700

$-0.97416100$

$-2.84581600$

H $\quad 5.51277300$

$-2.44720200$

$-1.37607400$

C $\quad 0.99358700$

1. 60321500

0. 14435200

C 2.25390000

1. 57307900

0. 77112900

C $\quad 0.66385700$

2. 76331200

$-0.57964700$

C $\quad 3.13980700$

2. 64366300

0. 67076600

H 2.55195200

0. 69968700

1. 33710900

C $\quad 1.55058100$

3. 83800600

$-0.67843100$

H $\quad-0.29717900$

2. 85337500

$-1.07337900$

C 2.79531600

3. 78549100

$-0.05491100$

H 4. 10540700

2. 58258000

1. 16602100

H 1.25817100
H

C $\quad-1.81586500$

H $\quad-0.17119700$

H $\quad-2.56792600$

\section{TS3-Ph}

$$
\text { C }
$$

C $\quad-2.03636000$

C -2.48090000

C $\quad-2.53388400$

N -1.83756600

S $\quad-2.65393300$

$-1.90472500$

0 -4.05998000

H $\quad-2.07214300$

H -3.55502000

H $\quad-3.41222100$

H -1.67164600

H $\quad-1.79312600$

H $\quad-3.51753900$

C $\quad-2.54090500$

H $\quad-3.04928900$

H -3.03417400

H -1.48832500

C $\quad-2.86255100$

C $\quad-0.52560700$

C $\quad 0.08967300$

C $\quad 0.78808200$

C $\quad-0.02854200$

C 1.36272900

H 0.89315600

C $\quad 0.54394500$

H $\quad-0.54955300$

C 1.23987200

H $\quad 1.90305400$

H $\quad 0.45237200$

H 1.68238000

C -3.84466300

C $\quad-3.72291400$

C -4.93072400

C $\quad-4.62672100$

H $\quad-2.87978100$

C $\quad-5.85041400$
4. 62104600

$-1.65581300$

$-2.92030800$

$-2.35716600$
$-0.12991300$

$-0.61005800$

$-0.89026100$

$-0.95092900$ 


\begin{tabular}{lrrr} 
H & -5.08613000 & -0.33741100 & -1.72443500 \\
C & -5.69723000 & 2.99553400 & -1.68651700 \\
H & -4.50625600 & 4.49083500 & -0.68130500 \\
H & -6.69168700 & 1.30037600 & -2.58299400 \\
H & -6.41637700 & 3.71454000 & -2.06821800 \\
C & -0.36805100 & -1.95011000 & -0.21459100 \\
H & 0.04887700 & -2.26873100 & -1.18171300 \\
H & -0.01163200 & -2.66687900 & 0.53272400 \\
Au & 2.24151000 & -0.39712300 & -0.22431600 \\
P & 4.56593300 & -0.20125400 & -0.59821300 \\
C & 5.04972500 & 1.25270400 & -1.62101100 \\
H & 4.71036900 & 2.17287000 & -1.13597000 \\
H & 6.13650800 & 1.29500900 & -1.75168400 \\
H & 4.57281500 & 1.18789200 & -2.60356400 \\
C & 5.56739300 & -0.02202000 & 0.93724600 \\
H & 5.41520100 & -0.89330100 & 1.58124700 \\
H & 6.63315800 & 0.06753500 & 0.70068100 \\
H & 5.24483200 & 0.86877500 & 1.48438000 \\
C & 5.32852900 & -1.63575700 & -1.46656900 \\
H & 6.40386300 & -1.48146100 & -1.60714100 \\
\hline & 5.17132000 & -2.54679900 & -0.88147500 \\
\hline
\end{tabular}

\section{INT4-Ph}

\begin{tabular}{|c|c|c|c|}
\hline C & -2.50872000 & -0.98583500 & -1.13752600 \\
\hline $\mathrm{C}$ & 0.25133700 & -0.47410100 & 0.02271000 \\
\hline $\mathrm{C}$ & -1.95087500 & 0.81070800 & 0.53498200 \\
\hline C & -2.62726900 & 0.35555700 & 1.99862100 \\
\hline C & -2.43813400 & 1. 76265000 & 1. 63770600 \\
\hline N & -1.78805100 & -1.97548600 & -0.35624200 \\
\hline S & -2.64372000 & -2.87513700 & 0.78002900 \\
\hline 0 & -1.92148400 & -2.84766200 & 2. 05462000 \\
\hline 0 & -4.03454300 & -2.40062400 & 0.69435000 \\
\hline $\mathrm{H}$ & -1.83729100 & -0.65856800 & -1.94679000 \\
\hline H & -3.38524300 & -1.42329400 & -1.61747700 \\
\hline H & -3.61750700 & -0.08646800 & 1.97533900 \\
\hline $\mathrm{H}$ & -1.88582500 & -0.19850800 & 2.56388600 \\
\hline $\mathrm{H}$ & -1.63191500 & 2. 30243400 & 2. 12110200 \\
\hline $\mathrm{H}$ & -3.32862200 & 2. 35386500 & 1. 46533400 \\
\hline $\mathrm{C}$ & -2.58652600 & -4.56242800 & 0.15489200 \\
\hline $\mathrm{H}$ & -3.12103000 & -5.18792400 & 0.87409200 \\
\hline $\mathrm{H}$ & -3.07422800 & -4.59533600 & -0.82073400 \\
\hline H & -1.54383300 & -4.87856200 & 0.08404500 \\
\hline $\mathrm{C}$ & -2.90156900 & 0.26294600 & -0.37568500 \\
\hline
\end{tabular}

$$
\begin{aligned}
& \text { C } \quad-0.44270000 \quad 0.63420500 \quad 0.38152600 \\
& \text { C } \quad 0.29979500 \quad 1.92037000 \quad 0.63112700 \\
& \begin{array}{llll}
\text { C } & 1.18071800 & 2.05076800 & 1.71780100
\end{array} \\
& \text { C } \quad 0.10956200 \quad 3.02944900 \quad-0.20994100 \\
& \text { C } \quad 1.86231000 \quad 3.24912900 \quad 1.94614100 \\
& \text { H } \quad 1.32516000 \quad 1.20652500 \quad 2.38649200 \\
& \begin{array}{llll}
\text { C } & 0.79672500 & 4.22397500 & 0.01107200
\end{array} \\
& \mathrm{H} \quad-0.56476600 \quad 2.94779200 \quad-1.05993200 \\
& \text { C } \quad 1.67486600 \quad 4.33812300 \quad 1.09201400 \\
& \text { H } \quad 2.53266000 \quad 3.33338600 \quad 2.79736600 \\
& \text { H } \quad 0.64754300 \quad 5.06519100 \quad-0.66034400 \\
& \text { H } \quad 2.20241300 \quad 5.27066500 \quad 1.27073200 \\
& \text { C } \quad-4.19154400 \quad 0.86084200 \quad-0.67166200 \\
& \begin{array}{llll}
\text { C } & -4.27833500 & 2.26168300 & -0.87300500
\end{array} \\
& \text { C } \quad-5.34780200 \quad 0.06728700 \quad-0.87468000 \\
& \begin{array}{llll}
\text { C } & -5.47401300 & 2.84051600 & -1.27793800
\end{array} \\
& \text { H } \quad-3.38947300 \quad 2.87470700 \quad-0.76624400 \\
& \text { C } \quad-6.54890800 \quad 0.66407600 \quad-1.23874100 \\
& \text { H } \quad-5.30631400 \quad-0.99730600 \quad-0.67329400 \\
& \text { C } \quad-6.61335500 \quad 2.04565600 \quad-1.44951100 \\
& \text { H } \quad-5.52184200 \quad 3.90932500 \quad-1.46161200 \\
& \text { H } \quad-7.43800600 \quad 0.05321700 \quad-1.35961200 \\
& \text { H } \quad-7.55116200 \quad 2.50403600 \quad-1.74945000 \\
& \text { C } \quad-0.31960900 \quad-1.84527100 \quad-0.25296300 \\
& \text { H } \quad 0.11143700 \quad-2.22264100 \quad-1.18996900 \\
& \text { H } \quad 0.01803300 \quad-2.52429000 \quad 0.53667400 \\
& \mathrm{Au} \quad 2.32433000 \quad-0.41514800 \quad-0.23413500 \\
& \text { P } \quad 4.66263000 \quad-0.37881200 \quad-0.56563300 \\
& \begin{array}{llll}
\text { C } & 5.25794700 & 1.02117400 & -1.60468200
\end{array} \\
& \text { H } \quad 4.97172100 \quad 1.97060600 \quad-1.14241800 \\
& \text { H } \quad 6.34707300 \quad 0.98990100 \quad-1.71721800 \\
& \text { H } \quad 4.79373700 \quad 0.96927300 \quad-2.59406300 \\
& \text { C } \quad 5.65168700 \quad-0.23935000 \quad 0.98239800 \\
& \text { H } \quad 5.43353000-1.08781800 \quad 1.63786000 \\
& \text { H } \quad 6.72450400 \quad-0.22317700 \quad 0.76180600 \\
& \text { H } \quad 5.38011800 \quad 0.67984400 \quad 1.50986700 \\
& \text { C } \quad 5.34277900-1.87517400 \quad-1.39766200 \\
& \text { H } \quad 6.42836100-1.79598900 \quad-1.52057900 \\
& \text { H } \quad 5.11399400 \quad-2.76426500 \quad-0.80247400 \\
& \text { H } \quad 4.87912000 \quad-1.99263300 \quad-2.38168600
\end{aligned}
$$

\section{TS4-Ph}

$\begin{array}{lrrr}\text { C } & -2.71154400 & -1.08299900 & -1.03883400 \\ \text { C } & 0.20891500 & -0.42620300 & -0.13200600\end{array}$ 


\begin{tabular}{|c|c|c|c|}
\hline C & -1.93769900 & 0.91796900 & 0.35449500 \\
\hline & -2.96571500 & 0.32480600 & 1. 70350200 \\
\hline & -2.49379800 & 1. 73418200 & 1. 49111600 \\
\hline & -1.80829100 & -2.00748200 & -0.38540600 \\
\hline & -2.40825300 & -2.93705400 & 0.88890500 \\
\hline & -1.53996100 & -2.75593500 & 2. 05828600 \\
\hline & -3.84281500 & -2.62812500 & 0.96539200 \\
\hline I & -2.26942300 & -0.81168900 & -2.00903800 \\
\hline & -3.65997800 & -1.57475200 & -1.25514000 \\
\hline H & -4.02878100 & 0.11485800 & 1. 73724400 \\
\hline $\mathrm{H}$ & -2.33125700 & -0.38834300 & 2. 22050400 \\
\hline H & -1.73986200 & 2. 11685700 & 2. 17156100 \\
\hline I & -3.28333000 & 2. 44710500 & 1. 27536900 \\
\hline 0 & -2.22133500 & -4.63545700 & 0.32358800 \\
\hline H & -2.57671700 & -5.27997900 & 1. 13130700 \\
\hline I & -2.82586500 & -4.77437700 & -0.57402700 \\
\hline H & -1.16655600 & -4.83352000 & 0.12267100 \\
\hline C & -2.98734500 & 0.21792300 & -0.29875200 \\
\hline 0 & -0.47521300 & 0.67735300 & 0.29534500 \\
\hline & 0.30879700 & 1. 89216400 & 0.72968400 \\
\hline 0 & 1. 10501300 & 1. 85823600 & 1. 88679300 \\
\hline & 0.24047000 & 3. 08760000 & -0.00278400 \\
\hline & 1. 82250400 & 2. 98661600 & 2. 29163700 \\
\hline & 1. 15534900 & 0.94273600 & 2. 47026000 \\
\hline & 0.96332700 & 4. 21355200 & 0.39688800 \\
\hline 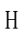 & -0.36640400 & 3. 13114200 & -0.90459500 \\
\hline & 1. 75554000 & 4. 16630900 & 1. 54647900 \\
\hline & 2. 42639800 & 2.94533600 & 3. 19413000 \\
\hline & 0.90702900 & 5. 12678700 & -0.18897400 \\
\hline & 2. 31101500 & 5. 04452000 & 1. 86278400 \\
\hline 0 & -4.27736400 & 0.86930700 & -0.67126900 \\
\hline 0 & -4.27576200 & 2. 16186700 & -1.22351100 \\
\hline 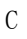 & -5.49166400 & 0.16642400 & -0.56588000 \\
\hline ( & -5.46746000 & 2. 74120800 & -1.65831000 \\
\hline & -3.33967600 & 2. 70280700 & -1.33102600 \\
\hline & -6.68038800 & 0.75729000 & -0.98792000 \\
\hline H & -5.50422300 & -0.83008800 & -0.13124400 \\
\hline 0 & -6.67051200 & 2. 04292500 & -1.53702100 \\
\hline & -5.45456400 & 3. 73600400 & -2.09337400 \\
\hline & -7.61511400 & 0.21375700 & -0.88933700 \\
\hline & -7.59813600 & 2. 49762300 & -1.87159000 \\
\hline $\mathrm{C}$ & -0.36244300 & -1.77151800 & -0.50471900 \\
\hline & -0.06849800 & -1.99002400 & -1.54203800 \\
\hline & 0.14564500 & -2.52175400 & 0.10895200 \\
\hline
\end{tabular}

$\begin{array}{lrrr}\mathrm{Au} & 2.28620800 & -0.36877000 & -0.32926700 \\ \mathrm{P} & 4.63307600 & -0.30752700 & -0.59877900 \\ \mathrm{C} & 5.25118500 & 1.23459100 & -1.39300600 \\ \mathrm{H} & 4.94996200 & 2.10143200 & -0.79737200 \\ \mathrm{H} & 6.34292500 & 1.21918800 & -1.48053400 \\ \mathrm{H} & 4.81348800 & 1.33594100 & -2.39063100 \\ \mathrm{C} & 5.57811000 & -0.41704200 & 0.97827500 \\ \mathrm{H} & 5.33604700 & -1.35330300 & 1.48992600 \\ \mathrm{H} & 6.65649500 & -0.37950000 & 0.79024100 \\ \mathrm{H} & 5.29917100 & 0.41394400 & 1.63298200 \\ \mathrm{C} & 5.32597700 & -1.66146900 & -1.63687400 \\ \mathrm{H} & 6.41395300 & -1.56884000 & -1.72315800 \\ \mathrm{H} & 5.08363300 & -2.63075200 & -1.19096300 \\ \mathrm{H} & 4.88339300 & -1.62349200 & -2.63672000\end{array}$

\section{INT5-Ph}

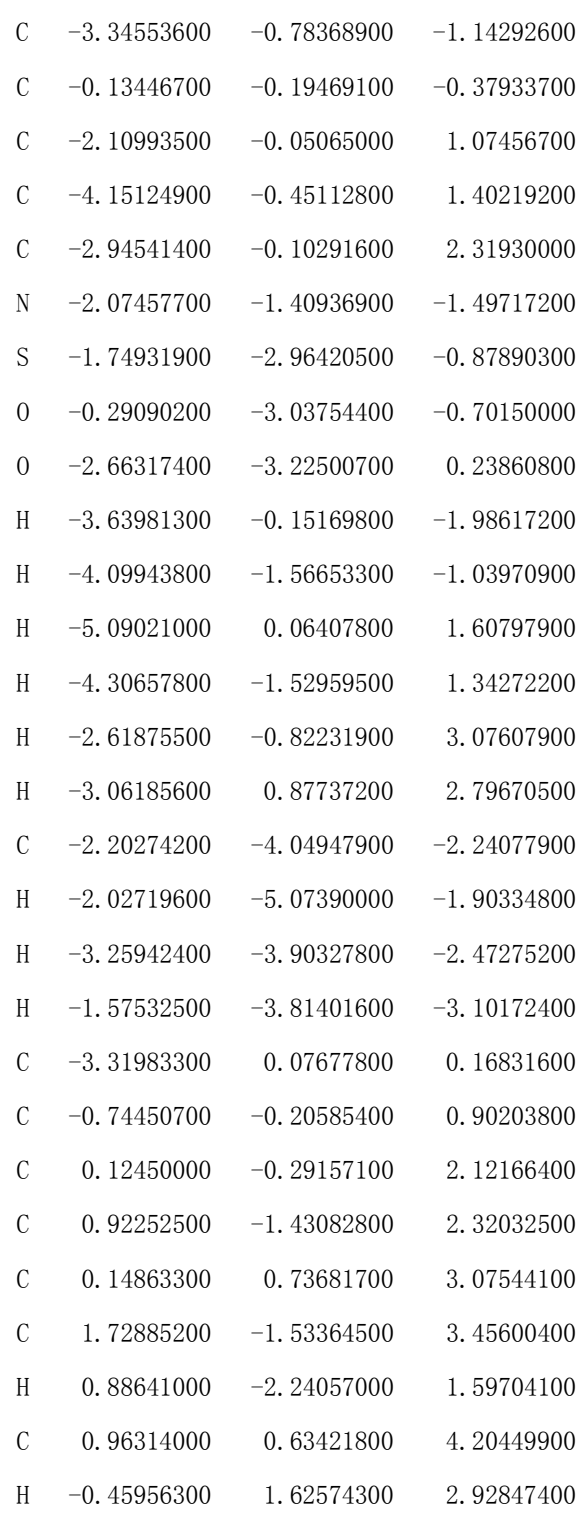




\begin{tabular}{|c|c|c|c|}
\hline C & 1. 75304000 & -0.50198300 & 4. 39762100 \\
\hline $\mathrm{H}$ & 2. 32970200 & -2.42575900 & 3. 60912400 \\
\hline $\mathrm{H}$ & 0.97612900 & 1. 43840700 & 4. 93465800 \\
\hline $\mathrm{H}$ & 2. 37807100 & -0.58573300 & 5. 28205900 \\
\hline $\mathrm{C}$ & -3.66548400 & 1. 54328300 & -0.15040600 \\
\hline $\mathrm{C}$ & -2.70622000 & 2.56238700 & -0.18278600 \\
\hline $\mathrm{C}$ & -4.99425800 & 1. 85783400 & -0.47716800 \\
\hline $\mathrm{C}$ & -3.06626300 & 3. 86794700 & -0.53175700 \\
\hline $\mathrm{H}$ & -1.67213900 & 2. 35171700 & 0.07425400 \\
\hline $\mathrm{C}$ & -5.35084800 & 3. 15779400 & -0.83243000 \\
\hline $\mathrm{H}$ & -5.75703200 & 1. 08340900 & -0.45607800 \\
\hline $\mathrm{C}$ & -4.38710600 & 4. 16945800 & -0.85856800 \\
\hline $\mathrm{H}$ & -2.30961200 & 4. 64748500 & -0.54250300 \\
\hline $\mathrm{H}$ & -6.38351800 & 3. 38104100 & -1.08452600 \\
\hline $\mathrm{H}$ & -4.66690500 & 5. 18339500 & -1.12834200 \\
\hline $\mathrm{C}$ & -0.90875000 & -0.52985300 & -1.63042700 \\
\hline H & -1.25107300 & 0.41119400 & -2.08861500 \\
\hline $\mathrm{H}$ & -0.21399800 & -0.96844800 & -2.35125000 \\
\hline $\mathrm{Au}$ & 1. 85969600 & 0.25779900 & -0.58497000 \\
\hline P & 4. 15778100 & 0.79584100 & -0.82860700 \\
\hline $\mathrm{C}$ & 4. 66993700 & 2. 29034400 & 0.11462600 \\
\hline $\mathrm{H}$ & 4. 45370400 & 2. 14743000 & 1. 17743500 \\
\hline $\mathrm{H}$ & 5. 74114300 & 2. 48030900 & -0.01181100 \\
\hline $\mathrm{H}$ & 4. 10825500 & 3. 16081600 & -0.23721100 \\
\hline $\mathrm{C}$ & 5. 28314700 & -0.53664100 & -0.24226600 \\
\hline $\mathrm{H}$ & 5. 10057600 & -1.45302300 & -0.81137000 \\
\hline H & 6.33018400 & -0.24008700 & -0.36627700 \\
\hline $\mathrm{H}$ & 5. 09042000 & -0.74366200 & 0.81449700 \\
\hline $\mathrm{C}$ & 4. 68731600 & 1. 12805100 & -2.55896600 \\
\hline H & 5. 75631600 & 1. 36300700 & -2.59854100 \\
\hline $\mathrm{H}$ & 4. 49130100 & 0.24967500 & -3.18107800 \\
\hline H & 4. 12042200 & 1. 97114000 & -2.96490800 \\
\hline
\end{tabular}

\section{TS5-Ph}

$\begin{array}{llll}\text { C } & 0.97222500 & -0.96222200 & -0.85012400 \\ \mathrm{~N} & 2.33216300 & -0.86787600 & -1.09807500 \\ \mathrm{C} & 0.00300900 & 0.03943300 & -0.51453200 \\ \mathrm{C} & 0.22817600 & 1.49072600 & -0.48825200 \\ \mathrm{C} & 2.99313400 & 0.38582100 & -1.47856500 \\ \mathrm{C} & 2.85946900 & 1.45854000 & -0.37889000 \\ \mathrm{C} & 1.45544000 & 2.03521100 & -0.28862100 \\ \mathrm{H} & 0.66500100 & -1.99175100 & -0.68685600 \\ \mathrm{H} & 0.17607900 & -0.62831300 & -1.78162900 \\ \mathrm{H} & 2.56426000 & 0.75652100 & -2.41776200\end{array}$

$$
\begin{aligned}
& \text { H } \quad 4.04362900 \\
& \text { C } \quad 1.95332300 \\
& \text { 0. } 16516100 \\
& \text { 3. } 45967800-0.31036800 \\
& \text { H } 1.40270800 \\
& \text { H } \quad 2.04662500 \\
& \text { 4. } 16347500 \\
& \text { 3. } 89196400 \\
& \text { 2. } 90092000 \\
& \text { C } \quad 3.31511900 \\
& \text { H } \quad 3.41933600 \\
& \text { H } \quad 4.21411600 \\
& \text { 2. } 98903800 \\
& \text { 3. } 28639300 \\
& \mathrm{Au} \quad-1.90101100 \\
& \text { P }-4.00430600 \\
& -0.66899500 \\
& -1.45213500 \\
& \text { C } \quad-4.25911700 \quad-3.25579300 \\
& \text { H } \quad-3.50096900 \\
& \text { H } \quad-5.25328000 \\
& \text { H } \quad-4.16264000 \\
& -3.81999200 \\
& -3.55743900 \\
& -3.49668200 \\
& \text { C } \quad-4.33437400 \\
& \text { H } \quad-4.27751800 \\
& \text { H } \quad-5.32867900 \\
& -1.17731200 \\
& -0.10845500 \\
& -1.54874300 \\
& -3.58242500 \\
& \text { C } \quad-5.41607200 \\
& \text { H } \quad-6.36895900 \\
& \text { H } \quad-5.37968100 \\
& \text { H } \quad-5.34950700 \\
& \text { S } \quad 3.17334100 \\
& 0 \quad 4.09077300 \\
& \text { 0 2. } 13248000 \\
& \text { C } 4.12006100 \\
& \text { H } \quad 4.71464600 \\
& \text { H } \quad 4.76617100 \\
& \text { 3. } 42706400 \\
& \text { C } \quad-0.97355000 \\
& -1.69776500 \\
& -0.65121700 \\
& -1.03860500 \\
& \text { 0. } 43083400 \\
& -0.84650200 \\
& -2.37443900 \\
& -2.09565400 \\
& -3.39729000 \\
& -2.63406700 \\
& -3.53626400 \\
& -1.77245500 \\
& -2.77466200 \\
& \text { 2. } 37937500 \\
& \text { C }-1.22897500 \\
& \text { C }-1.85766800 \\
& \text { 3. } 34680700 \\
& \text { 2. } 27663200 \\
& \text { 4. } 18831100 \\
& \text { C }-2.33870700 \\
& \text { H } \quad-0.56297300 \\
& \text { 3. } 43155500 \\
& \text { C }-2.96398100 \\
& \text { 3. } 12178600 \\
& \text { 1. } 54636100 \\
& \text { H } \quad-1.66847900 \\
& \text { C } \quad-3.20856100 \\
& \text { 4. } 07884800 \\
& \text { 4. } 92962800 \\
& \text { 3. } 04043200 \\
& -3.62689000 \\
& \text { H } \quad-4.06732200 \\
& \text { 4. } 73896200 \\
& \text { 0. } 99524500 \\
& \text { C } \quad 3.49134400 \\
& 0.96023500 \\
& \text { C } \quad 4.89127100 \\
& \text { 0. } 56891100 \\
& \text { 0. } 50157600 \\
& \text { C } \quad 5.50356000 \\
& \text { H } \quad 5.51027900 \\
& \text { 1. } 30107600 \\
& 0.69275000 \\
& -0.81937400 \\
& -1.90452000 \\
& -0.33647900 \\
& \text { 0. } 03928800 \\
& 0.73969200 \\
& \text { 0. } 48456800 \\
& \text { 1. } 03559200 \\
& \text { 0. } 83120700 \\
& \text {-0. } 57821900 \\
& \text { 2. } 52837400 \\
& \text { 2. } 75431900 \\
& \text { 2. } 79847800 \\
& \text { 3. } 12875700 \\
& \text {-0. } 12556400 \\
& \text { 0. } 25069800 \\
& \text { 0. } 03084400 \\
& \text {-1. } 19998200 \\
& -1.43354400 \\
& -2.53198600 \\
& -1.52148600 \\
& \text { 0. } 07378200 \\
& -0.09188600 \\
& \text { 0. } 24850300 \\
& \text { 0. } 90439700 \\
& -0.57631600 \\
& \text { 0. } 40815000 \\
& -1.66394000 \\
& \text { 0. } 30988600 \\
& \text { 1. } 26244900 \\
& -1.76539300 \\
& \text {-2. } 44703600 \\
& -0.77711200 \\
& \text { 1. } 08250500 \\
& \text {-2. } 62229500 \\
& -0.85757900 \\
& \text { 0. } 93049500 \\
& \text { 1. } 04165800 \\
& \text { 2. } 01945100 \\
& \text { 2. } 20847400
\end{aligned}
$$




$\begin{array}{llll}\text { C } & 3.33418300 & 0.11290000 & 3.19111800 \\ \text { H } & 1.63712800 & 0.60838200 & 1.96529200 \\ \text { C } & 4.72490600 & 0.07471800 & 3.28841800 \\ \text { H } & 6.58757100 & 0.48790000 & 2.27806800 \\ \text { H } & 2.72035700 & -0.20398200 & 4.02974700 \\ \text { H } & 5.20076000 & -0.27409600 & 4.20016000\end{array}$

\section{INT6-Ph}

$\begin{array}{lrrr}\text { C } & -0.35546800 & 0.13394300 & 2.67981100 \\ \text { C } & -2.01925200 & -1.53215700 & 2.17657700 \\ \text { C } & -1.31761200 & 0.90043600 & 3.34162800 \\ \text { H } & 0.66893400 & 0.49227900 & 2.63930500 \\ \text { C } & -2.98594500 & -0.75692800 & 2.82233000 \\ \text { H } & -2.32792100 & -2.47510300 & 1.74102200 \\ \text { C } & -2.64065500 & 0.45864100 & 3.41563500 \\ \text { H } & -1.02420000 & 1.83158300 & 3.82042600 \\ \text { H } & -4.00899900 & -1.11867400 & 2.87377300 \\ \text { H } & -3.38805700 & 1.04375400 & 3.94437000 \\ \text { Au } & -0.29466000 & 1.18126800 & -0.68640200 \\ \text { P } & -1.60698800 & 3.07664900 & -0.37165000 \\ \text { C } & -1.67853100 & 4.15551300 & -1.85741800 \\ \text { H } & -2.08826800 & 3.59508100 & -2.70275100 \\ \text { H } & -2.31171500 & 5.02850600 & -1.66510000 \\ \text { H } & -0.67191400 & 4.49353300 & -2.12042900 \\ \text { C } & -3.35326600 & 2.67357100 & 0.02857900 \\ \text { H } & -3.38964000 & 2.07667000 & 0.94451000 \\ \text { H } & -3.93424500 & 3.59215700 & 0.16608100 \\ \text { H } & -3.79157000 & 2.08764500 & -0.78453800 \\ \text { C } & -1.03048800 & 4.16670000 & 0.98938200 \\ \text { H } & -1.68924400 & 5.03639500 & 1.08752800 \\ \text { H } & -1.03108700 & 3.60501200 & 1.92732800 \\ \text { H } & -0.01157000 & 4.50930500 & 0.78678300\end{array}$

\section{Pro2-Ph}

$\begin{array}{rrrr}\text { N } & -1.20390600 & -1.43288300 & 0.29874900 \\ \text { C } & -0.19519800 & -1.87266200 & -0.56188200 \\ \text { C } & 1.06892800 & -1.40626400 & -0.70137500 \\ \text { C } & -0.92211500 & -0.59026700 & 1.46167300 \\ \text { C } & -0.30619700 & 0.78094900 & 1.08666800 \\ \text { C } & 1.14007800 & 0.68664300 & 0.63017700 \\ \text { C } & 1.77172400 & -0.28120900 & -0.07204900 \\ \text { H } & -0.49838300 & -2.72288200 & -1.16281500 \\ \text { H } & 1.66397100 & -1.97242800 & -1.41263800 \\ \text { H } & -1.85593300 & -0.46396100 & 2.01109100 \\ \text { H } & -0.21978600 & -1.12616300 & 2.11046500 \\ \text { C } & 0.22929700 & 1.59860700 & 2.32071400 \\ \text { H } & -0.31346700 & 2.51549800 & 2.56442700 \\ \text { H } & 0.30605300 & 0.97810400 & 3.21946500 \\ \text { C } & 1.59944800 & 1.76009600 & 1.59716500 \\ \text { H } & 1.70279000 & 2.75328900 & 1.14154700 \\ \text { H } & 2.50751500 & 1.55398900 & 2.17412400 \\ \text { C } & 3.25597100 & -0.25789700 & -0.22145200\end{array}$




\begin{tabular}{|c|c|c|c|}
\hline C & 4. 00637400 & -1.44277400 & -0.12069700 \\
\hline $\mathrm{C}$ & 3. 94583400 & 0.94239100 & -0.46324800 \\
\hline $\mathrm{C}$ & 5. 39542300 & -1.42598300 & -0.24481400 \\
\hline & 3. 49534200 & -2.38145300 & 0.07513200 \\
\hline & 5. 33485300 & 0.96026200 & -0.58887800 \\
\hline & 3. 38328200 & 1. 86410200 & -0.57775500 \\
\hline $\mathrm{C}$ & 6.06644200 & -0.22397500 & -0.47919300 \\
\hline $\mathrm{H}$ & 5. 95452900 & -2.35378600 & -0.15483000 \\
\hline & 5. 84508400 & 1. 90024700 & -0.78304100 \\
\hline $\mathrm{H}$ & 7. 14840200 & -0.21098200 & -0.57965500 \\
\hline S & -2.78684000 & -2.02651200 & 0.03543200 \\
\hline 0 & -3.54011400 & -1.76146100 & 1. 26175100 \\
\hline & -2.66318700 & -3.38074800 & -0.50703500 \\
\hline $\mathrm{C}$ & -3.45104400 & -0.98283200 & -1.27642900 \\
\hline H & -2.78688900 & -1.03979000 & -2.14054200 \\
\hline & -4.43480200 & -1.38826300 & -1.52596700 \\
\hline H & -3.53209100 & 0.04231800 & -0.91394700 \\
\hline $\mathrm{C}$ & -1.24513300 & 1.58292700 & 0.19266900 \\
\hline $\mathrm{C}$ & -2.43768900 & 2. 10536900 & 0.72005000 \\
\hline $\mathrm{C}$ & -0.96303800 & 1. 80484800 & -1.16101200 \\
\hline $\mathrm{C}$ & -3.32337700 & 2. 82459500 & -0.08331300 \\
\hline $\mathrm{H}$ & -2.67740900 & 1. 94893900 & 1. 76934200 \\
\hline $\mathrm{C}$ & -1.84692400 & 2.52706300 & -1.96788000 \\
\hline $\mathrm{H}$ & -0.04338100 & 1. 40953000 & -1.58133100 \\
\hline $\mathrm{C}$ & -3.03011500 & 3. 03862300 & -1.43356800 \\
\hline $\mathrm{H}$ & -4.23959300 & 3. 22159100 & 0.34602200 \\
\hline $\mathrm{H}$ & -1.60681400 & 2. 69058200 & -3.01540300 \\
\hline & -3.71647900 & 3. 60250800 & -2.0595290 \\
\hline
\end{tabular}

\section{INT1-H}

$\begin{array}{lrrr}\text { C } & -0.95561300 & 1.58176900 & 1.45762600 \\ \text { C } & -0.96895600 & 0.17937900 & 0.92414300 \\ \text { C } & -1.45385700 & -0.93372100 & 0.66540300 \\ \text { C } & -1.46370800 & 2.82855700 & -0.65816700 \\ \text { C } & -2.82009900 & 3.30766600 & -0.21577300 \\ \text { C } & -3.94977800 & 2.67802500 & -0.50966100 \\ \text { C } & -5.41498200 & 2.65016000 & -0.45274000 \\ \text { C } & -4.62182300 & 1.55361100 & -1.18338000 \\ \text { C } & -2.09777200 & -2.17878100 & 0.38973000 \\ \text { C } & -2.11043300 & -3.19836900 & 1.36452800 \\ \text { C } & -2.77503500 & -4.39255800 & 1.10431100 \\ \text { C } & -3.42390800 & -4.58241600 & -0.12012000 \\ \text { C } & -3.41216200 & -3.57575800 & -1.09114700 \\ \text { C } & -2.75280300 & -2.37576400 & -0.84434600\end{array}$

$$
\begin{aligned}
& \text { N } \quad-0.56723100 \quad 2.62727300 \quad 0.51259600 \\
& \text { S } \quad 1.09567400 \quad 2.83463600 \quad 0.24133800 \\
& \begin{array}{llll}
0 & 1.44257100 & 2.47546800 & -1.14297600
\end{array} \\
& \begin{array}{llll}
0 & 1.79369900 & 2.16637500 & 1.35240100
\end{array} \\
& \text { H } \quad-1.97589900 \quad 1.81441400 \quad 1.78045600 \\
& \text { H } \quad-0.29761200 \quad 1.62836400 \quad 2.32647400 \\
& \text { H } \quad-0.98885400 \quad 3.57439300 \quad-1.30414100 \\
& \text { H } \quad-1.54824600 \quad 1.90541300 \quad-1.24535300 \\
& \text { H } \quad-5.90036100 \quad 2.38573000 \quad 0.48534600 \\
& \text { H } \quad-5.97566500 \quad 3.36333000 \quad-1.05453200 \\
& \mathrm{H} \quad-4.65739000 \quad 1.53757800 \quad-2.27162400 \\
& \text { H } \quad-4.58930500 \quad 0.56501900 \quad-0.72660800 \\
& \text { H } \quad-1.60691700 \quad-3.03992900 \quad 2.31313200 \\
& \text { H } \quad-2.78923300 \quad-5.17585000 \quad 1.85576300 \\
& \text { H } \quad-3.94087900 \quad-5.51674100 \quad-0.31774600 \\
& \text { H } \quad-3.91847700 \quad-3.72757000 \quad-2.03937500 \\
& \text { H } \quad-2.73917200 \quad-1.58735400 \quad-1.59042200 \\
& \begin{array}{llll}
\text { C } & 1.30046900 & 4.61334200 & 0.42258900
\end{array} \\
& \text { H } \quad 1.02879800 \quad 4.89162500 \quad 1.44161400 \\
& \text { H } \quad 2.35310000 \quad 4.83072400 \quad 0.22627300 \\
& \text { H } \quad 0.67097200 \quad 5.12647200 \quad-0.30691300 \\
& \mathrm{Au} \quad 0.91126800 \quad-0.74543500 \quad 0.15498600 \\
& \text { P } \quad 3.10371000 \quad-1.08699800 \quad-0.54525900 \\
& \begin{array}{llll}
\text { C } & 4.29422900 & -0.40013800 & 0.67036700
\end{array} \\
& \text { H } \quad 4.22730400 \quad-0.95900600 \quad 1.60845900 \\
& \text { H } \quad 5.31641900 \quad-0.46718400 \quad 0.28254600 \\
& \text { H } \quad 4.04042800 \quad 0.64406000 \quad 0.87228200 \\
& \begin{array}{llll}
\text { C } & 3.59236100 & -2.84089500 & -0.79172500
\end{array} \\
& \text { H } \quad 2.95778600 \quad-3.30071500 \quad-1.55480500 \\
& \text { H } \quad 4.63822100 \quad-2.90013200 \quad-1.11184200 \\
& \text { H } \quad 3.47181300 \quad-3.39656000 \quad 0.14269900 \\
& \begin{array}{llll}
\text { C } & 3.45430100 & -0.23554800 & -2.13165400
\end{array} \\
& \text { H } \quad 4.51851400 \quad-0.31426000 \quad-2.37894300 \\
& \text { H } \quad 2.86445900 \quad-0.69071200 \quad-2.93288900 \\
& \text { H } \quad 3.16656100 \quad 0.81595500 \quad-2.04426100 \\
& \text { H } \quad-2.84511100 \quad 4.23609800 \quad 0.35447900
\end{aligned}
$$

\section{TS1-H}

$\begin{array}{lrrr}\text { C } & -3.10401300 & -0.13348200 & -1.66833400 \\ \text { C } & -0.47938700 & -0.23468000 & -0.33162400 \\ \text { C } & -3.15226700 & 1.64838800 & 0.13751200 \\ \text { C } & -3.69132700 & 1.34407700 & 1.46494000 \\ \text { C } & -3.33665500 & 2.77656100 & 1.05323400 \\ \text { N } & -2.75886500 & -1.20772900 & -0.74746400\end{array}$




\begin{tabular}{|c|c|c|c|}
\hline S & -3.92448700 & -1.87098900 & 0.28314100 \\
\hline 0 & -3.32543400 & -2.00070600 & 1. 61493500 \\
\hline c & -5.15481200 & -1.10933900 & 0.05867300 \\
\hline H & -2.53650800 & -0.27882000 & -2.59580500 \\
\hline $\mathrm{H}$ & -4.16600600 & -0.21072500 & -1.91802800 \\
\hline $\mathrm{H}$ & -4.73330100 & 1. 03643100 & 1. 51909100 \\
\hline $\mathrm{H}$ & -3.05110600 & 0.84297000 & 2. 18806100 \\
\hline $\mathrm{H}$ & -2.45669200 & 3.23426800 & 1. 50300100 \\
\hline $\mathrm{H}$ & -4.13697100 & 3. 48172900 & 0.83220200 \\
\hline $\mathrm{C}$ & -4.18044100 & -3.53414600 & -0.36247800 \\
\hline $\mathrm{H}$ & -4.92030000 & -4.01541300 & 0.28186700 \\
\hline $\mathrm{H}$ & -4.55567100 & -3.46104700 & -1.38462500 \\
\hline H & -3.23811000 & -4.08473500 & -0.32671600 \\
\hline $\mathrm{C}$ & -2.82551000 & 1. 25613300 & -1.11508900 \\
\hline $\mathrm{C}$ & -0.71102500 & 1. 03499300 & -0.36155400 \\
\hline C & -0.19138300 & 2. 37381200 & -0.22140000 \\
\hline C & 0.17431000 & 2. 85216200 & 1. 05541400 \\
\hline $\mathrm{C}$ & -0.00650200 & 3. 20202600 & -1.34671500 \\
\hline C & 0.72596900 & 4. 12420200 & 1. 19459100 \\
\hline $\mathrm{H}$ & 0.03595300 & 2. 21217500 & 1. 92191200 \\
\hline $\mathrm{C}$ & 0.53853500 & 4. 47375000 & -1.19757200 \\
\hline $\mathrm{H}$ & -0.28078100 & 2. 83693100 & -2.33189500 \\
\hline c & 0.90440000 & 4. 93664600 & 0.07113000 \\
\hline $\mathrm{H}$ & 1. 01151300 & 4. 48365100 & 2. 17869700 \\
\hline $\mathrm{H}$ & 0.68222800 & 5. 10484700 & -2.06931100 \\
\hline $\mathrm{H}$ & 1. 32775300 & 5.93044700 & 0.18317100 \\
\hline $\mathrm{C}$ & -1.34641700 & -1.46172700 & -0.50587100 \\
\hline $\mathrm{H}$ & -0.92585000 & -2.03625500 & -1.34443300 \\
\hline H & -1.25117200 & -2.08119700 & 0.38968300 \\
\hline $\mathrm{Au}$ & 1. 60362600 & -0.60344500 & -0.09522600 \\
\hline P & 3. 87058900 & -1.12537100 & 0.18687900 \\
\hline C & 4. 99220900 & -0.20225600 & -0.94107200 \\
\hline $\mathrm{H}$ & 4. 87273300 & 0.87367500 & -0.78386700 \\
\hline $\mathrm{H}$ & 6. 03583300 & -0.47836000 & -0.75561600 \\
\hline $\mathrm{H}$ & 4. 74068500 & -0.42907600 & -1.98125000 \\
\hline $\mathrm{C}$ & 4. 50288100 & -0.77239200 & 1. 87742100 \\
\hline $\mathrm{H}$ & 3. 93647800 & -1.35100300 & 2. 61300600 \\
\hline $\mathrm{H}$ & 5. 56357100 & -1.03480500 & 1. 95372000 \\
\hline $\mathrm{H}$ & 4. 37952900 & 0.29052400 & 2. 10491500 \\
\hline $\mathrm{C}$ & 4. 26177200 & -2.89838200 & -0.10330800 \\
\hline H & 5. 33090100 & -3.08394700 & 0.04556500 \\
\hline H & 3. 69054100 & -3.52324900 & 0.58947600 \\
\hline H & 3.98908600 & -3.17664100 & -1.12548500 \\
\hline $\mathrm{H}$ & 2. 59470500 & 2. 02653400 & -1.84987700 \\
\hline
\end{tabular}

\section{INT2-H}

C -3.12921200

C $\quad-0.60234700$

C $\quad-2.24238200$

0. 58917200

$-1.47550700$

$-0.05639200$

$-0.21503600$

C -2.86202400

1. 70299800

0. 66882400

C -2.34841200

1. 22016600

1. 92757700

N -2.99358100

2. 65113300

1. 80242600

S $\quad-4.31266900$

$-0.68145400$

$-0.77344400$

0 -3.75623300

$-1.32159000$

0. 08745100

$0 \quad-5.31623900$

$-1.98320400$

1. 27160000

H -2.75055900

$-0.26019700$

0. 19714500

H $\quad-4.18935600$

0. 49180300

$-2.50202700$

H $\quad-3.93206400$

0. 84931400

$-1.52625100$

H $\quad-2.30117900$

1. 03516600

1. 90845400

$-1.44934100$

0.54611500

2. 56872600

2. 35295600

H $\quad-3.06225100$

2. 91172300

3. 46518000

1. 69731400

C $\quad-4.97452900$

$-2.59224600$

$-1.00260100$

H $\quad-5.83282600$

$-3.03425800$

$-0.49052900$

H $\quad-5.28993400$

-2. 12673200

$-1.93814400$

H $\quad-4.20597900$

$-3.34773200$

$-1.17630100$

C $\quad-2.38206200$

1. 70108700

$-0.75532900$

C $\quad-0.92564200$

1. 31576300

$-0.19869800$

C 0.15900000

2. 35897000

$-0.23229300$

C $\quad 0.97140700$

2. 57652400

0. 89195000

C $\quad 0.38974200$

3. 11305300

$-1.38970400$

C 1.98821900

3. 53210100

0. 85921300

H $\quad 0.80453100$

1. 99753400

1. 79717300

C 1.40937700

4. 06744400

$-1.42406500$

H $\quad-0.22227700$

2. 95287000

-2. 27369300

C 2.20853000

4. 28103700

$-0.29976000$

H 2. 59910500

3. 70004900

1. 74180800

H 1.57559400

4. 64414600

$-2.32924300$

H 2.99406600

5. 03057100

$-0.32359400$

C $\quad-1.64294900$

$-1.10726700$

$-0.42214800$

H -1.23440000

$-1.78057700$

$-1.19633700$

H $\quad-1.69133700$

$-1.71989900$

0. 49046500

$\mathrm{Au} \quad 1.34770000$

$-0.72067400$

$-0.05561600$

P $\quad 3.58095300$

$-1.49587000$

0. 12054100

C $\quad 4.79275400$

$-0.44138600$

$-0.77548400$

H 4. 74788200

0. 58164400

$-0.39052800$

H $\quad 5.80913600$

$-0.82958400$

$-0.65017600$

H $\quad 4.54762900$

$-0.41841200$

$-1.84147100$

C 4.20156800

$-1.56183000$

1. 85152200 


$\begin{array}{lrrr}\text { H } & 3.58088200 & -2.24106000 & 2.44334500 \\ \text { H } & 5.23901100 & -1.91192200 & 1.87615400 \\ \text { H } & 4.14985200 & -0.56605200 & 2.30152100 \\ \text { C } & 3.84609500 & -3.19104300 & -0.54166900 \\ \text { H } & 4.89552000 & -3.48685500 & -0.43860900 \\ \text { H } & 3.21837200 & -3.90376500 & 0.00140700 \\ \text { H } & 3.56868500 & -3.22289200 & -1.59949200 \\ \text { H } & -2.35657400 & 2.66198800 & -1.26776900\end{array}$

\section{TS2-H}

\begin{tabular}{|c|c|c|c|}
\hline & -3.24107200 & 0.85571700 & -1.18224900 \\
\hline & -0.64608900 & 0.00608600 & -0.11459800 \\
\hline & -2.05387800 & 1. 89003000 & 0.88653700 \\
\hline & -2.66083500 & 1. 36795500 & 2. 14191100 \\
\hline & -2.17516200 & 2.81312600 & 2. 03834200 \\
\hline & -3.02136200 & -0.48623900 & -0.61568300 \\
\hline S & -4.36120400 & -1.41788900 & -0.04318000 \\
\hline 0 & -3.75456600 & -2.48346400 & 0.75096400 \\
\hline & -5.32660500 & -0.47508500 & 0.51510500 \\
\hline & -3.06177400 & 0.82233300 & -2.26467800 \\
\hline $\mathrm{H}$ & -4.28810600 & 1. 12076700 & -1.01189500 \\
\hline & -3.72939600 & 1. 16854800 & 2. 14923000 \\
\hline & -2.07682000 & 0.70115500 & 2. 77532900 \\
\hline H & -1.27805300 & 3. 07931400 & 2. 59037700 \\
\hline H & -2.91635500 & 3. 60629400 & 1. 97739200 \\
\hline 0 & -5.05458100 & -2.11662900 & -1.54916800 \\
\hline H & -5.93254800 & -2.69373900 & -1.24778400 \\
\hline H & -5.35194800 & -1.30577600 & -2.21716200 \\
\hline & -4.31140900 & -2.76581700 & -2.01504700 \\
\hline c & -2.33982700 & 1. 90165900 & -0.54793400 \\
\hline C & -0.92964100 & 1. 45928000 & -0.06916200 \\
\hline & 0.24420600 & 2. 38395400 & -0.26724200 \\
\hline 0 & 1. 13289100 & 2. 64343100 & 0.78770400 \\
\hline & 0.47643700 & 2. 98553400 & -1.51032800 \\
\hline & 2.22740200 & 3. 48984700 & 0.60220300 \\
\hline & 0.96314400 & 2. 18780100 & 1. 76019200 \\
\hline 0 & 1. 57222500 & 3. 83159600 & -1.69829300 \\
\hline & -0.20436400 & 2. 79604900 & -2.33648700 \\
\hline $\mathrm{C}$ & 2. 44890600 & 4. 08646000 & -0.64227500 \\
\hline & 2. 89704600 & 3. 69630900 & 1. 43263000 \\
\hline$H$ & 1. 73568800 & 4. 29478700 & -2.66708200 \\
\hline 1 & 3. 29452900 & 4. 75310900 & -0.78437900 \\
\hline 0 & -1.74194700 & -0.89194200 & -0.24403400 \\
\hline$H$ & -1.54828600 & -1.96041600 & -0.29513800 \\
\hline
\end{tabular}

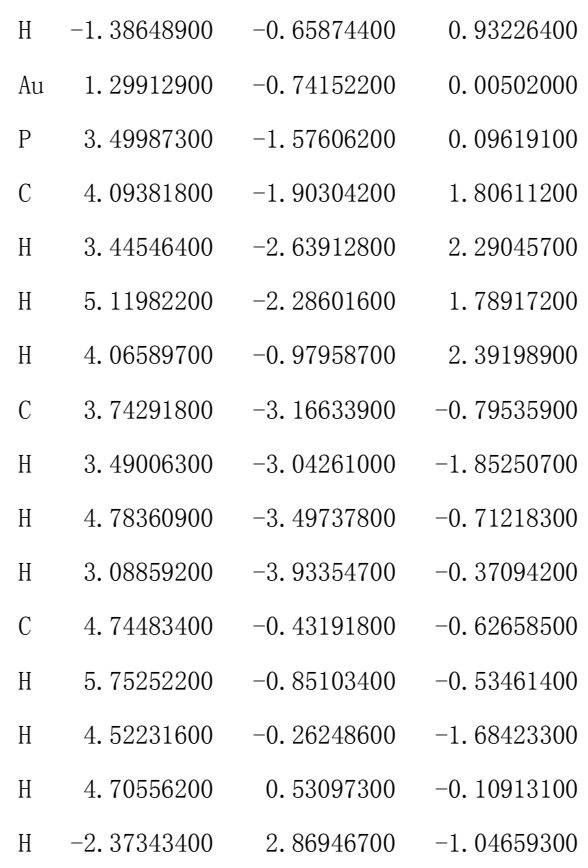

\section{INT3-H}

\begin{tabular}{|c|c|c|c|}
\hline $\mathrm{C}$ & -2.32782500 & -0.34294000 & 1. 36834700 \\
\hline $\mathrm{C}$ & -0.66515900 & -0.77988900 & -0.98286900 \\
\hline $\mathrm{C}$ & -2.09521400 & -2.62038300 & 0.04460800 \\
\hline $\mathrm{C}$ & -3.25269900 & -2.85077200 & -0.86149400 \\
\hline $\mathrm{C}$ & -2.60938100 & -4.00453600 & -0.09737700 \\
\hline $\mathrm{N}$ & -2.47262400 & 0.41994200 & 0.10776300 \\
\hline $\mathrm{S}$ & -3.31576000 & 1.96634600 & 0.21223900 \\
\hline 0 & -3.04462500 & 2. 48466500 & 1. 54814100 \\
\hline 0 & -2.94653900 & 2. 69342400 & -0.99869600 \\
\hline H & -1.70121300 & 0.24097100 & 2. 05311700 \\
\hline $\mathrm{H}$ & -3.31874900 & -0.43022900 & 1. 82609900 \\
\hline $\mathrm{H}$ & -4.23931000 & -2.51964000 & -0.54369000 \\
\hline $\mathrm{H}$ & -3.09255000 & -2.80338400 & -1.93672200 \\
\hline $\mathrm{H}$ & -2.01494400 & -4.71804600 & -0.66258900 \\
\hline $\mathrm{H}$ & -3.16222100 & -4.43960800 & 0.73191000 \\
\hline $\mathrm{C}$ & -5.03761100 & 1. 45956000 & 0.10114900 \\
\hline $\mathrm{H}$ & -5.62899600 & 2. 37391300 & 0.19768100 \\
\hline $\mathrm{H}$ & -5.20478600 & 0.99485200 & -0.87169200 \\
\hline $\mathrm{H}$ & -5.27017400 & 0.77693800 & 0.92047100 \\
\hline $\mathrm{C}$ & -1.70937900 & -1.71463900 & 1. 15196800 \\
\hline $\mathrm{C}$ & -0.74937500 & -1.93479000 & -0.01859700 \\
\hline $\mathrm{C}$ & 0.51640400 & -2.73223400 & 0.16007600 \\
\hline $\mathrm{C}$ & 0.96460400 & -3.56485100 & -0.87660400 \\
\hline $\mathrm{C}$ & 1. 28199000 & -2.64294100 & 1. 33033600 \\
\hline $\mathrm{C}$ & 2. 15095200 & -4.28809900 & -0.74706700 \\
\hline & 0.37774800 & -3.65504800 & -1.78813500 \\
\hline
\end{tabular}




\begin{tabular}{|c|c|c|c|}
\hline C & 2. 46785100 & -3.37014300 & 1. 46423800 \\
\hline $\mathrm{H}$ & 0.94480100 & -2.00964700 & 2. 14740700 \\
\hline $\mathrm{C}$ & 2. 90599300 & -4.19253300 & 0.42483100 \\
\hline $\mathrm{H}$ & 2. 48029000 & -4.93376800 & -1.55618600 \\
\hline H & 3. 04267000 & -3.30044400 & 2. 38371700 \\
\hline $\mathrm{H}$ & 3. 82416800 & -4.76309800 & 0.52969000 \\
\hline $\mathrm{C}$ & -1.60863200 & 0.25065600 & -0.91929200 \\
\hline $\mathrm{H}$ & -0.30152900 & -1.00435200 & -1.98731300 \\
\hline $\mathrm{H}$ & -1.72478400 & 0.95686200 & -1.73598200 \\
\hline $\mathrm{H}$ & -1.43421000 & -2.18424500 & 2. 09511100 \\
\hline $\mathrm{Al}$ & 0.92555700 & 0.70006200 & -0.40466300 \\
\hline P & 2. 71746300 & 2. 09098100 & 0.17137800 \\
\hline C & 4. 34448500 & 1. 23985400 & 0. 10013900 \\
\hline $\mathrm{H}$ & 4. 52898400 & 0.87567400 & -0.91477000 \\
\hline $\mathrm{H}$ & 5. 14809100 & 1. 92672600 & 0.38680900 \\
\hline $\mathrm{H}$ & 4. 34359600 & 0.38229000 & 0.77913900 \\
\hline $\mathrm{C}$ & 2. 88391400 & 3.55844400 & -0.92207600 \\
\hline $\mathrm{H}$ & 1. 97103500 & 4. 15943700 & -0.87625400 \\
\hline $\mathrm{H}$ & 3. 73489200 & 4. 17235000 & -0.60779300 \\
\hline $\mathrm{H}$ & 3. 03615000 & 3. 23740800 & -1.95662700 \\
\hline $\mathrm{C}$ & 2.59868800 & 2. 77110900 & 1. 87476300 \\
\hline $\mathrm{H}$ & 3. 45737500 & 3. 41693600 & 2. 08797200 \\
\hline $\mathrm{H}$ & 1. 67802200 & 3. 35281200 & 1. 97872100 \\
\hline $\mathrm{H}$ & 2. 57684300 & 1. 95365400 & 2. 60140200 \\
\hline
\end{tabular}

\section{Sub-H}

$\begin{array}{rrrr}\text { C } & 1.13418200 & -0.03733100 & 1.52938000 \\ \text { C } & -0.27593100 & -0.13816200 & 1.13467300 \\ \text { C } & -1.43131900 & -0.23204200 & 0.77761800 \\ \text { C } & 1.94669900 & 0.79008500 & -0.68312000 \\ \text { C } & 2.29638600 & 2.17583200 & -0.20853100 \\ \text { C } & 1.46469000 & 3.20497100 & -0.28466100 \\ \text { C } & 1.20860400 & 4.63290000 & -0.05588800 \\ \text { C } & 0.13920000 & 3.71157400 & -0.66921300 \\ \text { C } & -2.78366900 & -0.37550600 & 0.33784600 \\ \text { C } & -3.82326100 & 0.35358900 & 0.94625900 \\ \text { C } & -5.13696200 & 0.20354400 & 0.50762600 \\ \text { C } & -5.43366800 & -0.66939900 & -0.54216600 \\ \text { C } & -4.40772200 & -1.39601200 & -1.15249400 \\ \text { C } & -3.09172400 & -1.25504000 & -0.71869500 \\ \text { N } & 2.10330300 & -0.19129400 & 0.41907700 \\ \text { S } & 2.34592800 & -1.80452200 & -0.09345600 \\ \text { 0 } & 1.71235200 & -2.04734100 & -1.39533100 \\ \text { O } & 2.05235200 & -2.66619100 & 1.05589300\end{array}$

\begin{tabular}{rrrr} 
H & 1.33169900 & 0.94668400 & 1.96893800 \\
H & 1.37484300 & -0.79266000 & 2.28151600 \\
H & 2.62550200 & 0.48944300 & -1.48907300 \\
H & 0.92875800 & 0.76394200 & -1.08963200 \\
H & 1.05650600 & 4.98368600 & 0.96408900 \\
H & 1.68501400 & 5.36521500 & -0.70649200 \\
H & -0.09251600 & 3.83421500 & -1.72628800 \\
H & -0.72110000 & 3.44348400 & -0.05803200 \\
H & -3.58919900 & 1.03068500 & 1.76226100 \\
H & -5.93153200 & 0.76962000 & 0.98639300 \\
H & -6.45933600 & -0.78361900 & -0.88231900 \\
H & -4.63364800 & -2.07724000 & -1.96839200 \\
H & -2.28990600 & -1.81840400 & -1.18598000 \\
C & 4.13261800 & -1.81281000 & -0.34917100 \\
H & 4.62187700 & -1.60752800 & 0.60354700 \\
H & 4.39122900 & -2.81061800 & -0.71147800 \\
H & 4.40266500 & -1.06479300 & -1.09693000 \\
H & 3.29480800 & 2.31038600 & 0.20835600 \\
& & & \\
\hline
\end{tabular}

\section{Pro1-H}

\begin{tabular}{|c|c|c|c|}
\hline & 1. 13317700 & -0.39700900 & 1. 29906300 \\
\hline & -0.26695200 & -0.55938900 & -1.22157700 \\
\hline & -0.56815400 & 1. 40900300 & 0.39261700 \\
\hline 0 & 0.12844300 & 2.58911500 & -0.17440200 \\
\hline c & -1.17265900 & 2. 74693700 & 0.61038200 \\
\hline 1 & 1. 80771700 & -0.42267400 & -0.01386700 \\
\hline S & 3. 49256500 & -0.53012100 & -0.06125100 \\
\hline 0 & 3. 94379700 & -0.90148300 & 1. 28089800 \\
\hline 0 & 3. 86503100 & -1.30991400 & -1.24333300 \\
\hline $\mathrm{H}$ & 1. 04754000 & -1.42062100 & 1. 68869900 \\
\hline $\mathrm{H}$ & 1. 75890800 & 0.16329300 & 1. 99856100 \\
\hline & 1. 04848200 & 2. 92923600 & 0.29769900 \\
\hline $\mathrm{H}$ & 0.10006400 & 2. 73726500 & -1.25192900 \\
\hline $\mathrm{H}$ & -2.07480800 & 3. 00086900 & 0.05795800 \\
\hline $\mathrm{H}$ & -1.12069600 & 3. 18815700 & 1. 60409700 \\
\hline C & 4. 00545400 & 1. 17478300 & -0.35953800 \\
\hline $\mathrm{H}$ & 5. 09689500 & 1. 18081000 & -0.41340200 \\
\hline $\mathrm{H}$ & 3.57384100 & 1. 50927300 & -1.30460200 \\
\hline $\mathrm{H}$ & 3. 66292100 & 1. 79829400 & 0.46838700 \\
\hline C & -0.25653500 & 0.21312000 & 1. 20612400 \\
\hline C & -1.04134100 & 0.05667300 & -0.10291600 \\
\hline C & -2.51490000 & -0.25206800 & -0.07074000 \\
\hline C & -3.36881900 & 0.33708000 & -1.01594800 \\
\hline $\mathrm{C}$ & -3.06392000 & -1.14282600 & 0.85971500 \\
\hline
\end{tabular}




$\begin{array}{lrrr}\text { C } & -4.73401900 & 0.05006400 & -1.02529500 \\ \text { H } & -2.95690400 & 1.02598300 & -1.75002100 \\ \text { C } & -4.43057900 & -1.43056800 & 0.85548100 \\ \text { H } & -2.41585500 & -1.62128800 & 1.58943100 \\ \text { C } & -5.27031300 & -0.83413800 & -0.08618400 \\ \text { H } & -5.37957200 & 0.51900800 & -1.76346600 \\ \text { H } & -4.83727600 & -2.12492400 & 1.58620900 \\ \text { H } & -6.33384500 & -1.05724300 & -0.09014200 \\ \text { C } & 1.05800000 & -0.75224400 & -1.16256300 \\ \text { H } & -0.80169100 & -0.85476600 & -2.11811700 \\ \text { H } & 1.63123000 & -1.18118900 & -1.97511000 \\ \text { H } & -0.82620600 & 0.08846300 & 2.12699700\end{array}$

\section{TS3-H}

\begin{tabular}{|c|c|c|c|}
\hline r & 3. 16353700 & -0.06435800 & 1. 73756500 \\
\hline & 0.58124900 & -0.16161300 & 0.17008800 \\
\hline & 2. 43907200 & 1. 68138400 & 0.00818600 \\
\hline & 3. 23451100 & 1. 73894100 & -1.36102900 \\
\hline & 2. 78434400 & 2. 98260000 & -0.69244100 \\
\hline & 2. 89737900 & -1.09864600 & 0.76362300 \\
\hline & 4. 14373500 & -1.46088400 & -0.33441500 \\
\hline 0 & 3. 56478300 & -1.52895800 & -1.67709600 \\
\hline 0 & 5. 23432800 & -0.52781200 & -0.01481300 \\
\hline & 2. 49673100 & -0.18233500 & 2. 60838800 \\
\hline & 4. 17920500 & -0.16851400 & 2. 14339000 \\
\hline H & 4. 27596500 & 1. 44043800 & -1.31984500 \\
\hline & 2. 66305400 & 1. 32621900 & -2.18641000 \\
\hline 4 & 1. 95631300 & 3.53066000 & -1.12938300 \\
\hline$H$ & 3.54206800 & 3. 60178800 & -0.21556300 \\
\hline C & 4. 66953600 & -3.11810700 & 0.13091000 \\
\hline 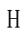 & 5. 46961900 & -3.40094800 & -0.55770900 \\
\hline$H$ & 5. 03760300 & -3.09871800 & 1. 15795600 \\
\hline $\mathrm{H}$ & 3. 82339200 & -3.80005400 & 0.02726100 \\
\hline C & 3. 06701600 & 1. 32707300 & 1. 21787600 \\
\hline $\mathrm{C}$ & 0.96761200 & 1. 12817800 & 0.05823700 \\
\hline $\mathrm{C}$ & -0.01289200 & 2. 26585900 & 0.03161700 \\
\hline $\mathrm{C}$ & -0.71591700 & 2.55625800 & -1.14997900 \\
\hline $\mathrm{C}$ & -0.23031300 & 3. 06557200 & 1. 16284800 \\
\hline $\mathrm{C}$ & -1.64041100 & 3. 60335700 & -1.18348500 \\
\hline $\mathrm{H}$ & -0.54506700 & 1. 95113000 & -2.03616300 \\
\hline$C$ & -1.15232100 & 4. 11166700 & 1. 12801300 \\
\hline$H$ & 0.31758500 & 2. 86082600 & 2. 08033000 \\
\hline $\mathrm{C}$ & -1.86096500 & 4. 38281400 & -0.04610900 \\
\hline & -2.17959500 & 3. 81449900 & -2.1028380 \\
\hline
\end{tabular}

$$
\begin{aligned}
& \text { H } \quad-1.31657000 \quad 4.71568400 \quad 2.01577900 \\
& \text { H } \quad-2.57235100 \quad 5.20290200 \quad-0.07574800 \\
& \begin{array}{llll}
\text { C } & 1.48821700 & -1.34879400 & 0.39131300
\end{array} \\
& \text { H } \quad 1.04851200 \quad-1.96747900 \quad 1.18603900 \\
& \text { H } \quad 1.48635800 \quad-1.96531600 \quad-0.51332000 \\
& \mathrm{Au} \quad-1.44182400 \quad-0.67124000 \quad 0.05961400 \\
& \text { P } \quad-3.71553100 \quad-1.29811200 \quad-0.06119900 \\
& \begin{array}{llll}
\text { C } & -4.33435600 & -2.19701800 & 1.42155200
\end{array} \\
& \text { H } \quad-4.22227000 \quad-1.56720100 \quad 2.30910300 \\
& \text { H } \quad-5.39012700 \quad-2.46293100 \quad 1.30195500 \\
& \text { H } \quad-3.75028100 \quad-3.10982900 \quad 1.57166800 \\
& \text { C } \quad-4.88208200 \quad 0.11327100 \quad-0.25512400 \\
& \text { H } \quad-4.65147100 \quad 0.65944800 \quad-1.17446600 \\
& \text { H } \quad-5.91767100 \quad-0.24087700 \quad-0.29855500 \\
& \text { H } \quad-4.77268000 \quad 0.80101000 \quad 0.58862600 \\
& \text { C } \quad-4.11213000 \quad-2.41545300 \quad-1.47014100 \\
& \text { H } \quad-5.17637100 \quad-2.67462000 \quad-1.47472500 \\
& \text { H } \quad-3.85922400 \quad-1.92336800 \quad-2.41400100 \\
& \text { H } \quad-3.52069900 \quad-3.33259900 \quad-1.39263600 \\
& \text { H } \quad 3.51849700 \quad 2.09888000 \quad 1.85052200
\end{aligned}
$$

\begin{tabular}{|c|c|c|c|}
\hline & -3.41832400 & -0.37629000 & -1.73775400 \\
\hline & -0.63949700 & -0.19485900 & -0.39085200 \\
\hline 0 & -2.47087500 & 1. 60819100 & -0.45951900 \\
\hline 0 & -3.04048500 & 2. 57196000 & 0.74630900 \\
\hline 0 & -2.74888000 & 3. 23025900 & -0.48853800 \\
\hline 1 & -2.92811900 & -1.36542200 & -0.77011500 \\
\hline$S$ & -3.88430900 & -1.47656700 & 0.63917400 \\
\hline 0 & -3.00913300 & -1.66599300 & 1. 79377500 \\
\hline 0 & -4.82624800 & -0.33493900 & 0.57225400 \\
\hline $\mathrm{H}$ & -2.68171600 & -0.33661000 & -2.55150400 \\
\hline & -4.37609000 & -0.68523600 & -2.16197200 \\
\hline $\mathrm{H}$ & -4.05637400 & 2. 34126900 & 1. 04658400 \\
\hline H & -2.30182000 & 2. 59613200 & 1. 54093200 \\
\hline $\mathrm{H}$ & -1.81124300 & 3. 76088700 & -0.60392800 \\
\hline H & -3.54912500 & 3.53600800 & -1.15315500 \\
\hline C & -4.83751200 & -2.98000900 & 0.38136900 \\
\hline $\mathrm{H}$ & -5.50335800 & -3.08552000 & 1. 24134400 \\
\hline $\mathrm{H}$ & -5.41501800 & -2.88304900 & -0.53951100 \\
\hline $\mathrm{H}$ & -4.14525000 & -3.82171200 & 0.32625800 \\
\hline C & -3.52793700 & 0.96663100 & -1.09463300 \\
\hline C & -1.06106800 & 1. 09755100 & -0.30822200 \\
\hline & -0.03550500 & 2. 15727400 & 0.01344900 \\
\hline
\end{tabular}

\section{INT4-H}




\begin{tabular}{|c|c|c|c|}
\hline C & 0.38600000 & 2. 35997000 & 1. 33817900 \\
\hline $\mathrm{C}$ & 0.52634200 & 2. 95542500 & -0.99794600 \\
\hline $\mathrm{C}$ & 1. 33875800 & 3. 33545500 & 1. 64443800 \\
\hline $\mathrm{H}$ & -0.02088600 & 1. 73216100 & 2. 12740500 \\
\hline $\mathrm{C}$ & 1. 48378100 & 3.92548200 & -0.69372200 \\
\hline $\mathrm{H}$ & 0.22236700 & 2. 80141700 & -2.03095800 \\
\hline $\mathrm{C}$ & 1. 88862500 & 4. 12137000 & 0.62968200 \\
\hline $\mathrm{H}$ & 1. 65002400 & 3. 47957100 & 2. 67542100 \\
\hline H & 1. 91296000 & 4. 52775200 & -1.48967700 \\
\hline $\mathrm{H}$ & 2. 62713300 & 4. 88167000 & 0.86708000 \\
\hline $\mathrm{C}$ & -1.45678700 & -1.44574100 & -0.61983500 \\
\hline $\mathrm{H}$ & -1.08074100 & -1.95443500 & -1.51659100 \\
\hline $\mathrm{H}$ & -1.26877500 & -2.12844800 & 0.21338600 \\
\hline $\mathrm{Au}$ & 1. 38941200 & -0.65483700 & -0.17026800 \\
\hline $\mathrm{P}$ & 3. 67451100 & -1.20014800 & 0.06673500 \\
\hline $\mathrm{C}$ & 4. 34992400 & -0.90921600 & 1. 75586300 \\
\hline H & 3. 79375800 & -1.50724000 & 2. 48393200 \\
\hline H & 5. 41009800 & -1.18078300 & 1. 80346600 \\
\hline $\mathrm{H}$ & 4. 23741700 & 0.14612300 & 2. 02127800 \\
\hline $\mathrm{C}$ & 4. 08946400 & -2.95998500 & -0.28301100 \\
\hline H & 3. 80934100 & -3.20961600 & -1.31070800 \\
\hline H & 5. 16149400 & -3.14083800 & -0.14958200 \\
\hline $\mathrm{H}$ & 3. 52977800 & -3.61276300 & 0.39350300 \\
\hline $\mathrm{C}$ & 4. 79650900 & -0.24278900 & -1.03651900 \\
\hline $\mathrm{H}$ & 5.84250700 & -0.52045000 & -0.86747600 \\
\hline H & 4. 53908800 & -0.43585000 & -2.08225800 \\
\hline $\mathrm{H}$ & 4. 67363200 & 0.82745800 & -0.84548400 \\
\hline H & -4.48681500 & 1. 47288200 & -1.14428500 \\
\hline
\end{tabular}

\section{TS4-H}

$\begin{array}{lrrr}\text { C } & -3.32738600 & -0.30569000 & -1.80947700 \\ \text { C } & -0.61745800 & -0.15960800 & -0.34095800 \\ \text { C } & -2.41069000 & 1.66753800 & -0.49069300 \\ \text { C } & -3.36665900 & 2.06107700 & 0.95317300 \\ \text { C } & -2.79361000 & 3.06804800 & 0.09280400 \\ \text { N } & -2.91680700 & -1.29035900 & -0.80091400 \\ \text { S } & -3.96729900 & -1.43594100 & 0.53315700 \\ \text { O } & -3.21211100 & -1.22282800 & 1.77237600 \\ \text { O } & -5.12910800 & -0.58597500 & 0.21755600 \\ \text { H } & -2.55095000 & -0.30797600 & -2.58660100 \\ \mathrm{H} & -4.27311600 & -0.59575300 & -2.26836000 \\ \mathrm{H} & -4.41934500 & 1.80304700 & 0.93244200 \\ \mathrm{H} & -2.79422700 & 1.62189500 & 1.76413800 \\ \mathrm{H} & -1.92139100 & 3.60556000 & 0.44691500\end{array}$

$$
\begin{aligned}
& \text { H } \quad-3.47083700 \quad 3.65593900 \quad-0.52134300 \\
& \text { C } \quad-4.48403900-3.15621000 \quad 0.46631900 \\
& \text { H } \quad-5.16044000 \quad-3.31697700 \quad 1.30939900 \\
& \text { H } \quad-4.99698400 \quad-3.33157800 \quad-0.48042800 \\
& \text { H } \quad-3.60215500 \quad-3.79267300 \quad 0.56064000 \\
& \text { C } \quad-3.42984700 \quad 1.06045900 \quad-1.20679900 \\
& \text { C } \quad-1.02016800 \quad 1.14044200 \quad-0.28401300 \\
& \begin{array}{llll}
\text { C } & 0.00475400 & 2.21279700 & -0.01971100
\end{array} \\
& \begin{array}{llll}
\text { C } & 0.61234700 & 2.33227900 & 1.24131300
\end{array} \\
& \text { C } \quad 0.36151100 \quad 3.12644400 \quad-1.02543300 \\
& \begin{array}{llll}
\text { C } & 1.55820700 & 3.33159800 & 1.48620800
\end{array} \\
& \text { H } \quad 0.34132500 \quad 1.63541000 \quad 2.03018900 \\
& \text { C } \quad 1.31206300 \quad 4.11987700 \quad-0.78394100 \\
& \text { H } \quad-0.09935800 \quad 3.04934500 \quad-2.00765400 \\
& \begin{array}{llll}
\text { C } & 1.91203700 & 4.22610300 & 0.47387000
\end{array} \\
& \text { H } \quad 2.01192700 \quad 3.41433400 \quad 2.47013300 \\
& \text { H } \quad 1.58357500 \quad 4.81086100 \quad-1.57713400 \\
& \text { H } \quad 2.64494400 \quad 5.00478700 \quad 0.66442600 \\
& \text { C } \quad-1.45969800 \quad-1.39783000 \quad-0.55940900 \\
& \text { H } \quad-1.04380000-1.94863500 \quad-1.41279300 \\
& \text { H } \quad-1.32506200 \quad-2.04986300 \quad 0.30899200 \\
& \mathrm{Au} \quad 1.40609400 \quad-0.64801200 \quad-0.13364400 \\
& \text { P } \quad 3.68597000 \quad-1.23323400 \quad 0.05894400 \\
& \begin{array}{llll}
\text { C } & 4.32355300 & -1.24887500 & 1.78695800
\end{array} \\
& \text { H } \quad 3.75514400 \quad-1.96834700 \quad 2.38387900 \\
& \text { H } \quad 5.38377600 \quad-1.52284800 \quad 1.80928900 \\
& \text { H } \quad 4.19992200 \quad-0.25829700 \quad 2.23456400 \\
& \text { C } \quad 4.10263500 \quad-2.90576800 \quad-0.58991600 \\
& \text { H } \quad 3.84002600 \quad-2.96628600 \quad-1.65029700 \\
& \text { H } \quad 5.17148000-3.11502700 \quad-0.47335300 \\
& \text { H } \quad 3.52872500 \quad-3.66489900 \quad-0.05008500 \\
& \text { C } \quad 4.83104800 \quad-0.10226000 \quad-0.83608000 \\
& \text { H } \quad 5.87191500 \quad-0.41879400 \quad-0.70896800 \\
& \text { H } \quad 4.58616600 \quad-0.09733100 \quad-1.90240600 \\
& \text { H } \quad 4.71585800 \quad 0.91648600 \quad-0.45419600 \\
& \text { H } \quad-4.37548900 \quad 1.58456900 \quad-1.31654500
\end{aligned}
$$

\section{INT5-H}

$\begin{array}{lrrr}\text { C } & -3.74375400 & -1.13417300 & -1.41256000 \\ \text { C } & -0.58275500 & -0.35200800 & -0.58998200 \\ \text { C } & -2.45795500 & 1.15999500 & -1.04178600 \\ \text { C } & -4.52446700 & 1.47188300 & -1.40888700 \\ \text { C } & -3.32272200 & 2.39152900 & -1.04344300 \\ \text { N } & -2.76044400 & -1.69647700 & -0.48573700\end{array}$




\begin{tabular}{|c|c|c|c|}
\hline$S$ & -3.05248700 & -1.47811600 & 1. 18367000 \\
\hline 0 & -1.73067300 & -1.39083900 & 1.82215400 \\
\hline 0 & -4.04516700 & -0.41049200 & 1. 35020200 \\
\hline H & -3.72845400 & -1.75597200 & -2.31359400 \\
\hline $\mathrm{H}$ & -4.73687700 & -1.23397900 & -0.96968500 \\
\hline $\mathrm{H}$ & -5.18614100 & 1. 80929100 & -2.20825800 \\
\hline $\mathrm{H}$ & -5.10430300 & 1. 20199700 & -0.52431400 \\
\hline $\mathrm{H}$ & -3.34546500 & 2. 94554100 & -0.09936800 \\
\hline $\mathrm{H}$ & -3.06482300 & 3. 09573500 & -1.84402800 \\
\hline $\mathrm{C}$ & -3.81168900 & -3.03567600 & 1. 66700300 \\
\hline $\mathrm{H}$ & -4.04199000 & -2.95959600 & 2. 73250600 \\
\hline $\mathrm{H}$ & -4.72835700 & -3.17526800 & 1.09089800 \\
\hline H & -3.10351000 & -3.84445600 & 1. 48120200 \\
\hline $\mathrm{C}$ & -3.49651600 & 0.35069000 & -1.77731900 \\
\hline $\mathrm{C}$ & -1.21918300 & 0.91147300 & -0.47841800 \\
\hline C & -0.49364600 & 2. 04277100 & 0.18681900 \\
\hline $\mathrm{C}$ & -0.16520900 & 1. 93912900 & 1. 54889700 \\
\hline $\mathrm{C}$ & -0.14850500 & 3. 20699800 & -0.51518500 \\
\hline $\mathrm{C}$ & 0.49762800 & 2.98603100 & 2. 19291400 \\
\hline $\mathrm{H}$ & -0.45299100 & 1. 04896300 & 2. 10131800 \\
\hline $\mathrm{C}$ & 0.52165700 & 4. 24786100 & 0.13037100 \\
\hline $\mathrm{H}$ & -0.38839400 & 3. 29402700 & -1.57196000 \\
\hline C & 0.84401300 & 4. 13985200 & 1. 48553700 \\
\hline $\mathrm{H}$ & 0.73310500 & 2. 90327600 & 3. 25025700 \\
\hline $\mathrm{H}$ & 0.78766200 & 5. 14338000 & -0.42394500 \\
\hline $\mathrm{H}$ & 1. 35593700 & 4. 95488400 & 1. 98905700 \\
\hline $\mathrm{C}$ & -1.34881100 & -1.61959700 & -0.88191100 \\
\hline $\mathrm{H}$ & -1.29540300 & -1.81269700 & -1.96526700 \\
\hline H & -0.81086400 & -2.44985400 & -0.41739800 \\
\hline $\mathrm{Au}$ & 1. 45236600 & -0.51467700 & -0.34298700 \\
\hline P & 3. 79984100 & -0.70774600 & -0.05874000 \\
\hline $\mathrm{C}$ & 4. 34168000 & -0.40922400 & 1. 67420200 \\
\hline $\mathrm{H}$ & 3.87394700 & -1.14028700 & 2. 34034700 \\
\hline $\mathrm{H}$ & 5. 43049800 & -0.49118600 & 1. 75900300 \\
\hline $\mathrm{H}$ & 4. 03094900 & 0.59134100 & 1. 98896300 \\
\hline $\mathrm{C}$ & 4. 48838100 & -2.35790300 & -0.49175900 \\
\hline $\mathrm{H}$ & 4. 27096200 & -2.58642500 & -1.53938900 \\
\hline H & 5. 57267800 & -2.37819600 & -0.33896400 \\
\hline H & 4. 02496200 & -3.12778800 & 0.13233900 \\
\hline $\mathrm{C}$ & 4. 77355900 & 0.48610300 & -1.06473900 \\
\hline H & 5. 84568100 & 0.36384500 & -0.87705200 \\
\hline H & 4. 57571600 & 0.32395300 & -2.12842900 \\
\hline H & 4. 47933300 & 1. 50881500 & -0.81130000 \\
\hline $\mathrm{H}$ & -3.26906200 & 0.42779400 & -2.85179600 \\
\hline
\end{tabular}

\section{TS5-H}

C -1.72042000

N $\quad-3.07285900 \quad-0.61982800$

0. 12339400

0. 39481600

C $\quad-0.63982600$

0. 13979000

0. 05031700

C -0.76402800

1. 60263800

$-0.05004300$

C $\quad-3.66409100$

0.67001300

0. 77117900

C $\quad-3.37026400$

1. 76612300

C -1.93934500

2. 24208400

$-0.25622300$

$-1.27931800$

$-0.52192000$

H $\quad-1.42826800$

$-1.84726100$

H $\quad-4.74293300$

0. 52972700

-1. 02876900

H $\quad-3.27020100$

0. 95688300

0. 16561300

0. 86519800

H $\quad-3.73637000$

1. 44015700

1. 75188900

C $\quad-2.34587200$

3. 69797900

$-1.23782500$

H -1.82105200

4. 36818000

-0. 29617400

H $\quad-2.29378500$

4. 13250000

0. 39158200

C $\quad-3.79237900$

3. 23414600

H $\quad-4.01872300$

3. 37774200

0. 06871100

H $\quad-4.60801600$

3. 64401100

1. 12942000

$\mathrm{Au} \quad 1.28931500$

$-0.70612300$

$-0.52945200$

P 3.43824800

$-1.65753100$

0. 00113000

C $\quad 4.42937000$

-1. 37687400

$-0.07459600$

H $\quad 3.92182600$

$-1.82266800$

1. 44962300

2. 31019300

H $\quad 5.42445600$

$-1.82349900$

1. 35009300

H 4.53485200

-0. 30291000

1. 62867400

C 4.48145500

$-0.99968100$

$-1.43925600$

H 5.47420000

$-1.46205200$

-1. 42229300

H 4.00604500

$-1.20803100$

$-2.40230200$

H 4.58702700

0. 08410000

$-1.33445100$

C $\quad 3.44828000$

$-3.48138900$

$-0.31293300$

H $\quad 4.47540600$

$-3.86020600$

$-0.34486600$

H 2.91306100

$-3.96615300$

0. 50888800

H 2. 94405500

$-3.73559200$

$-1.24993900$

S $\quad-4.14989600$

$-1.85586500$

$-0.21489900$

$0 \quad-3.28330400$

$-2.84596700$

$-0.84874700$

$0 \quad-5.20572700 \quad-1.15955000$

$-0.94329400$

C $\quad-4.84299300$

$-2.55605700$

1. 29141300

H $\quad-4.03864200$

$-3.00899800$

1. 87310000

H $\quad-5.35197100$

$-1.77323300$

1. 85673000

H $\quad-5.56209400$

$-3.31571000$

0. 97396300

C $\quad 0.49822000$

2. 40721300

0. 03476000

C 0.93052600

3. 17544000

$-1.05658900$

C 1.25295000

2. 43049000

1. 21999700 


$\begin{array}{llll}\text { C } & 2.09519200 & 3.94141700 & -0.97029000 \\ \text { H } & 0.35676100 & 3.16535800 & -1.97951000 \\ \text { C } & 2.41370000 & 3.20165200 & 1.30812300 \\ \text { H } & 0.91648400 & 1.86176400 & 2.08359100 \\ \text { C } & 2.83884700 & 3.95647600 & 0.21167500 \\ \text { H } & 2.41720400 & 4.52998000 & -1.82463400 \\ \text { H } & 2.97637400 & 3.22488400 & 2.23736800 \\ \text { H } & 3.73871800 & 4.56073900 & 0.28184300\end{array}$

\section{INT6-H}

\begin{tabular}{|c|c|c|c|}
\hline N & 2. 28551000 & -1.14914900 & -0.25575700 \\
\hline $\mathrm{C}$ & 1. 30306200 & -0.77854000 & -1.12160500 \\
\hline $\mathrm{C}$ & -0.07553200 & -1.04544400 & -1.10346300 \\
\hline & 2. 03169200 & -1.80947200 & 1. 04270700 \\
\hline & 1. 20693100 & -3.08273300 & 0.87023700 \\
\hline C & -0.25546100 & -2.90090800 & 0.51894100 \\
\hline $\mathrm{C}$ & -0.85746800 & -1.99780500 & -0.27225900 \\
\hline & 1. 68231300 & -0.21807900 & -1.97104400 \\
\hline H & -0.54748300 & -0.79529200 & -2.05514200 \\
\hline H & 2. 99897200 & -2.02891900 & 1.50084700 \\
\hline$\dot{\theta}$ & 1.51511500 & -1.10416500 & 1. 70525200 \\
\hline H & 1. 72119700 & -3.75991200 & 0.17575100 \\
\hline $\mathrm{C}$ & 0.73799100 & -3.84055000 & 2. 15320300 \\
\hline H & 1. 24731200 & -4.78073700 & 2. 37572700 \\
\hline$\theta$ & 0.76481000 & -3.20304600 & 3. 04276700 \\
\hline$C$ & -0.69576600 & -3.93295500 & 1. 53985400 \\
\hline H & -0.89024400 & -4.91260000 & 1. 08579100 \\
\hline 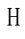 & -1.54070600 & -3.68697600 & 2. 18772200 \\
\hline $\mathrm{C}$ & -2.34585100 & -1.90638600 & -0.39585900 \\
\hline C & -3.14380900 & -1.63426000 & 0.72687800 \\
\hline $\mathrm{C}$ & -2.97300100 & -2.08405400 & -1.63975100 \\
\hline $\mathrm{C}$ & -4.53249900 & -1.54739800 & 0.61021500 \\
\hline H & -2.67227600 & -1.48662600 & 1. 69503700 \\
\hline $\mathrm{C}$ & -4.36076100 & -1.99701700 & -1.75631200 \\
\hline H & -2.37599200 & -2.31754800 & -2.51844300 \\
\hline C & -5.14437000 & -1.72771900 & -0.63178800 \\
\hline $\mathrm{H}$ & -5.13557000 & -1.34084500 & 1. 49009400 \\
\hline H & -4.83026700 & -2.14900400 & -2.72401600 \\
\hline H & -6.22473400 & -1.66355300 & -0.72268200 \\
\hline S & 3.82592700 & -0.29811000 & -0.41310500 \\
\hline 0 & 4. 14374100 & 0.21763900 & 0.91406200 \\
\hline 0 & 3. 68187700 & 0.57916900 & -1.57217000 \\
\hline C & 4. 95357600 & -1.63911700 & -0.82242800 \\
\hline & 4. 65792900 & -2.06503900 & -1.7824270 \\
\hline
\end{tabular}

$$
\begin{array}{lrrr}
\mathrm{H} & 5.94807900 & -1.19032400 & -0.89033200 \\
\mathrm{H} & 4.93563200 & -2.38741900 & -0.02804500 \\
\mathrm{Au} & -0.27844500 & 1.06196800 & -0.24955200 \\
\mathrm{P} & -0.72012500 & 3.19210700 & 0.59764400 \\
\mathrm{C} & 0.69687600 & 3.91512900 & 1.51710100 \\
\mathrm{H} & 1.57407200 & 3.96888400 & 0.86582100 \\
\mathrm{H} & 0.44754100 & 4.92167600 & 1.87013700 \\
\mathrm{H} & 0.94351700 & 3.28432500 & 2.37602100 \\
\mathrm{C} & -1.12584400 & 4.41054400 & -0.71565000 \\
\mathrm{H} & -2.01248500 & 4.08237200 & -1.26583800 \\
\mathrm{H} & -1.32052400 & 5.39375900 & -0.27385100 \\
\mathrm{H} & -0.29124800 & 4.49149600 & -1.41805200 \\
\mathrm{C} & -2.14031300 & 3.24269500 & 1.76249500 \\
\mathrm{H} & -2.31134700 & 4.26722800 & 2.11015200 \\
\mathrm{H} & -3.04258800 & 2.87735600 & 1.26352100 \\
\mathrm{H} & -1.93707600 & 2.60119000 & 2.62488700
\end{array}
$$

\begin{tabular}{|c|c|c|c|}
\hline & -1.95996700 & -0.15109400 & -0.10746000 \\
\hline $\mathrm{C}$ & -1.04389800 & -1.13474700 & -0.50732000 \\
\hline & 0.30754100 & -1.08791200 & -0.55462900 \\
\hline & -1.55756000 & 1. 07296600 & 0.59527100 \\
\hline & -0.52184200 & 1. 87597900 & -0.20143100 \\
\hline & 0.87244900 & 1. 29080200 & -0.23642800 \\
\hline C & 1. 26315700 & 0.00038200 & -0.30624300 \\
\hline H & -1.53376800 & -2.04128500 & -0.84234900 \\
\hline & 0.75781700 & -2.01791600 & -0.89161600 \\
\hline H & -2.45560400 & 1. 67426100 & 0.74951700 \\
\hline H & -1.13316100 & 0.81366700 & 1. 57456300 \\
\hline H & -0.93592900 & 2. 10927100 & -1.190 \\
\hline C & 2. 69160500 & -0.38579300 & -0.13513300 \\
\hline C & 3. 03702100 & -1.55511900 & 0.56589500 \\
\hline C & 3.73265400 & 0.40085700 & -0.65797200 \\
\hline C & 4. 37127400 & -1.91658100 & 0.74969200 \\
\hline $\mathrm{H}$ & 2. 24925500 & -2.17523100 & 0.98506500 \\
\hline $\mathrm{C}$ & 5. 06740600 & 0.04020000 & -0.47530000 \\
\hline $\mathrm{H}$ & 3. 49021200 & 1. 28732900 & -1.23555500 \\
\hline $\mathrm{C}$ & 5. 39366200 & -1.11946600 & 0.23092000 \\
\hline $\mathrm{H}$ & 4. 61239600 & -2.82145700 & 1. 30174400 \\
\hline $\mathrm{H}$ & 5.85414200 & 0.66130400 & -0.89593000 \\
\hline $\mathrm{H}$ & 6.43366900 & -1.40195200 & 0.37054400 \\
\hline $\mathrm{C}$ & 1. 51515900 & 2. 60271700 & 0.17719600 \\
\hline $\mathrm{H}$ & 2. 24285800 & 2.56467400 & 0.99642000 \\
\hline & 1. 97563100 & 3. 15274900 & -0.65390500 \\
\hline
\end{tabular}

\section{Pro2-H}




$\begin{array}{rrrr}\text { C } & 0.08333200 & 3.13052600 & 0.50601800 \\ \text { H } & -0.12155100 & 3.14178700 & 1.58185400 \\ \text { H } & -0.19241200 & 4.10194600 & 0.08678700 \\ \text { S } & -3.61790800 & -0.53675400 & -0.15986800 \\ \text { O } & -4.34851900 & 0.72800000 & -0.23602400 \\ \text { O } & -3.78992000 & -1.58715500 & -1.16199900 \\ \text { C } & -3.95318600 & -1.26633300 & 1.45960600 \\ \text { H } & -5.01315000 & -1.53091000 & 1.48091600 \\ \text { H } & -3.33630200 & -2.15920000 & 1.57940400 \\ \text { H } & -3.73293900 & -0.53095400 & 2.23618100\end{array}$

\section{INT2-Me}

\begin{tabular}{|c|c|c|c|}
\hline & 2. 66655500 & 0.62649400 & -0.93422200 \\
\hline & -0.06763000 & 0.23750000 & 0.25317900 \\
\hline & 2. 18082800 & -0.22263600 & 1. 39257000 \\
\hline & 2. 56375400 & 0.91783300 & 2. 29415500 \\
\hline & 2. 77839300 & -0.50291600 & 2. 74946300 \\
\hline & 1. 83955700 & 1. 72851000 & -0.50197400 \\
\hline & 2.50416600 & 3. 24686800 & -0.20460900 \\
\hline & 1. 66004900 & 3. 88359300 & 0.80603200 \\
\hline & 3. 93857600 & 3. 02324400 & 0.00186400 \\
\hline & 2. 36584800 & 0.29204900 & -1.93522200 \\
\hline & 3. 70400600 & 0.98092100 & -1.00167300 \\
\hline & 3. 43700800 & 1. 50423600 & 2. 02568500 \\
\hline & 1. 75752800 & 1. 49249900 & 2. 73904100 \\
\hline & 2. 12604300 & -0.90167100 & 3.52122400 \\
\hline & 3. 80135700 & -0.86607400 & 2. 79771200 \\
\hline & 2. 31182200 & 4. 17559200 & -1.73912500 \\
\hline & 2. 72064100 & 5. 17348500 & -1.56230700 \\
\hline & 2. 87011100 & 3. 67480100 & -2.53253300 \\
\hline & 1. 24996400 & 4. 24490700 & -1.98465100 \\
\hline & 2. 67113900 & -0.52853800 & 0.05553600 \\
\hline 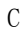 & 0.73576500 & -0.59217600 & 0.99685800 \\
\hline & 3. 17968700 & -1.82119400 & -0.35666000 \\
\hline & 3. 43626800 & -2.84588000 & 0.59310200 \\
\hline & 3. 48950200 & -2.09270500 & -1.71693000 \\
\hline$C$ & 3. 96951900 & -4.06789000 & 0.20306700 \\
\hline & 3. 23176700 & -2.66928500 & 1. 63965200 \\
\hline & 4. 02787500 & -3.31283500 & -2.09831000 \\
\hline & 3. 30957000 & -1.34974500 & -2.48398800 \\
\hline r & 4. 26825900 & -4.30613100 & -1.14135000 \\
\hline & 4. 16213500 & -4.83324700 & 0.94835500 \\
\hline & 4. 25911700 & -3.49626600 & -3.14285900 \\
\hline & 4. 68945300 & -5.26029600 & -1.44404500 \\
\hline
\end{tabular}

$$
\begin{aligned}
& \text { C } \quad 0.39577600 \quad 1.50768300 \quad-0.42123100 \\
& \text { H } \quad-0.04294800 \quad 1.52015200 \quad-1.43324900 \\
& \text { H } \quad-0.04220800 \quad 2.35720900 \quad 0.11704800 \\
& \mathrm{Au} \quad-2.11134500 \quad-0.09061300 \quad 0.01932100 \\
& \text { P } \quad-4.44885900 \quad-0.38086300 \quad-0.23420900 \\
& \text { C } \quad-4.93127300 \quad-1.84756600 \quad-1.23756400 \\
& \text { H } \quad-4.54618800 \quad-2.75929400 \quad-0.77116700 \\
& \text { H } \quad-6.02126600 \quad-1.92015200 \quad-1.31751900 \\
& \text { H } \quad-4.50328700 \quad-1.76723400 \quad-2.24121200 \\
& \text { C } \quad-5.35668300 \quad-0.59325600 \quad 1.35318400 \\
& \text { H } \quad-5.20081000 \quad 0.28331300 \quad 1.98902200 \\
& \text { H } \quad-6.42963300 \quad-0.71838500 \quad 1.17211800 \\
& \text { H } \quad-4.97843700 \quad-1.47296000 \quad 1.88237400 \\
& \text { C } \quad-5.29082700 \quad 1.03380500 \quad-1.05827900 \\
& \text { H } \quad-6.36750500 \quad 0.85006400 \quad-1.14020900 \\
& \text { H } \quad-5.12724600 \quad 1.94894200 \quad-0.48147200 \\
& \text { H } \quad-4.87561700 \quad 1.17935800 \quad-2.05996200 \\
& \begin{array}{llll}
\text { C } & 0.26967400 & -1.93869700 & 1.49888500
\end{array} \\
& \text { H } \quad 0.28549900 \quad-1.97942100 \quad 2.59461800 \\
& \text { H } \quad-0.74588200 \quad-2.15035500 \quad 1.15734400 \\
& \text { H } \quad 0.91714700 \quad-2.74925600 \quad 1.14106000
\end{aligned}
$$

\section{TS2-Me}

$\begin{array}{lrrr}\text { C } & 2.70414200 & 0.63264800 & -0.74293600 \\ \text { C } & -0.07183000 & 0.23004800 & 0.05151700 \\ \text { C } & 1.93819000 & -0.34214500 & 1.51352900 \\ \text { C } & 2.00472300 & 0.72428700 & 2.55128600 \\ \text { C } & 2.24516500 & -0.73883100 & 2.91075600 \\ \text { N } & 1.77529800 & 1.75477500 & -0.53930200 \\ \text { S } & 2.37555300 & 3.34886000 & -0.24315600 \\ \text { O } & 1.21046000 & 4.09895500 & 0.22074100 \\ \text { O } & 3.58745500 & 3.20629900 & 0.55879100 \\ \text { H } & 2.67375800 & 0.32656300 & -1.79562700 \\ \text { H } & 3.71276600 & 0.98886500 & -0.51756900 \\ \text { H } & 2.85078900 & 1.40636000 & 2.52958900 \\ \text { H } & 1.08018000 & 1.17521800 & 2.91166400 \\ \text { H } & 1.46989600 & -1.24607600 & 3.47990300 \\ \text { H } & 3.25493300 & -1.05319100 & 3.15861000 \\ \mathrm{C} & 2.84574300 & 3.92674400 & -1.88126100 \\ \mathrm{H} & 3.26816000 & 4.92601800 & -1.74805900 \\ \mathrm{H} & 3.60006900 & 3.25481900 & -2.29572300 \\ \mathrm{H} & 1.95726300 & 3.96854700 & -2.51347100 \\ \mathrm{C} & 2.40010200 & -0.58552600 & 0.13861600 \\ \mathrm{C} & 0.88472200 & -0.80113500 & 0.50284900\end{array}$




\begin{tabular}{|c|c|c|c|}
\hline C & 3. 25946000 & -1.76515000 & -0.23193000 \\
\hline & 4. 42565400 & -2.05470800 & 0.48969900 \\
\hline C & 2. 95335000 & -2.53774000 & -1.36304800 \\
\hline C & 5. 25758000 & -3.10628000 & 0.10096100 \\
\hline $\mathrm{H}$ & 4. 69183300 & -1.45092100 & 1. 35218100 \\
\hline $\mathrm{C}$ & 3. 78219200 & -3.59015800 & -1.75026900 \\
\hline H & 2. 05683100 & -2.31909000 & -1.93903200 \\
\hline $\mathrm{C}$ & 4. 93586900 & -3.87785500 & -1.01675600 \\
\hline $\mathrm{H}$ & 6. 15726500 & -3.31982700 & 0.67071400 \\
\hline $\mathrm{H}$ & 3.52867300 & -4.18467900 & -2.62329500 \\
\hline $\mathrm{H}$ & 5. 58210300 & -4.69729500 & -1.31741800 \\
\hline $\mathrm{C}$ & 0.42752100 & 1. 51199400 & -0.31045200 \\
\hline $\mathrm{H}$ & -0.26171500 & 2. 28396200 & -0.64301900 \\
\hline H & 0.05864200 & 1. 39012400 & 0.88421900 \\
\hline $\mathrm{Au}$ & -2.13530700 & -0.09604000 & -0.03414500 \\
\hline $\mathrm{P}$ & -4.46209000 & -0.46688900 & -0.15273000 \\
\hline $\mathrm{C}$ & -4.94620100 & -2.22193800 & 0.10863600 \\
\hline $\mathrm{H}$ & -4.60462000 & -2.55871400 & 1. 09188400 \\
\hline $\mathrm{H}$ & -6.03415400 & -2.33360200 & 0.05069600 \\
\hline $\mathrm{H}$ & -4.48095000 & -2.85374700 & -0.65377200 \\
\hline $\mathrm{C}$ & -5.42271300 & 0.47939900 & 1. 09823500 \\
\hline $\mathrm{H}$ & -5.25506600 & 1.55194100 & 0.96285000 \\
\hline $\mathrm{H}$ & -6.49291500 & 0.26878400 & 0.99933200 \\
\hline $\mathrm{H}$ & -5.09576000 & 0.20351600 & 2. 10508500 \\
\hline $\mathrm{C}$ & -5.20727200 & -0.00446500 & -1.76944600 \\
\hline $\mathrm{H}$ & -6.28637500 & -0.19151100 & -1.76403200 \\
\hline $\mathrm{H}$ & -5.02982600 & 1. 05634300 & -1.96967100 \\
\hline $\mathrm{H}$ & -4.74722600 & -0.58906200 & -2.57159900 \\
\hline $\mathrm{C}$ & 0.34123700 & -2.21232500 & 0.66995600 \\
\hline $\mathrm{H}$ & -0.47087300 & -2.22073200 & 1. 40677600 \\
\hline $\mathrm{H}$ & -0.05054600 & -2.61007700 & -0.27263400 \\
\hline $\mathrm{H}$ & 1. 12239000 & -2.89045000 & 1. 01955000 \\
\hline
\end{tabular}

\section{TS3-Me}

$\begin{array}{lrrr}\text { C } & 2.39322700 & 0.60762400 & -1.04039700 \\ \text { C } & -0.30678300 & 0.10736100 & 0.21258200 \\ \text { C } & 1.99231500 & -0.56625000 & 1.15359000 \\ \text { C } & 2.37045400 & 0.42400500 & 2.29469900 \\ \text { C } & 2.55219500 & -1.04367200 & 2.47992800 \\ \text { N } & 1.59191600 & 1.66815900 & -0.47990600 \\ \text { S } & 2.36833400 & 3.03929500 & 0.11209200 \\ \text { O } & 1.65354200 & 3.47972000 & 1.31024600 \\ 0 & 3.79728100 & 2.69681400 & 0.14619900 \\ \text { H } & 1.83622300 & 0.11278400 & -1.85066100\end{array}$

\begin{tabular}{|c|c|c|c|}
\hline $\mathrm{H}$ & 3. 28625000 & 1. 02731700 & -1.50622900 \\
\hline $\mathrm{H}$ & 3. 25228300 & 1. 04128000 & 2. 15644600 \\
\hline H & 1. 52109500 & 0.93425200 & 2. 73679600 \\
\hline $\mathrm{H}$ & 1. 86704100 & -1.56402100 & 3. 14142400 \\
\hline H & 3.57119000 & -1.41654900 & 2. 51683000 \\
\hline $\mathrm{C}$ & 2. 13115200 & 4. 30170600 & -1.15307700 \\
\hline H & 2. 61676400 & 5. 21187600 & -0.79260800 \\
\hline $\mathrm{H}$ & 2.59316100 & 3. 96785300 & -2.08399500 \\
\hline $\mathrm{H}$ & 1. 06102400 & 4. 47468600 & -1.28443200 \\
\hline $\mathrm{C}$ & 2. 80013100 & -0.46831300 & -0.04931400 \\
\hline $\mathrm{C}$ & 0.50069100 & -0.79918700 & 0.80674400 \\
\hline $\mathrm{C}$ & 3. 88384800 & -1.34725100 & -0.37575400 \\
\hline $\mathrm{C}$ & 3. 90957700 & -2.67165700 & 0.14797300 \\
\hline $\mathrm{C}$ & 4. 93435000 & -0.94870200 & -1.25182500 \\
\hline $\mathrm{C}$ & 4. 91224000 & -3.55635500 & -0.21566100 \\
\hline $\mathrm{H}$ & 3. 10728400 & -3.00149700 & 0.79616900 \\
\hline $\mathrm{C}$ & 5.95523900 & -1.82880200 & -1.57426000 \\
\hline $\mathrm{H}$ & 4. 98437100 & 0.06910400 & -1.61981400 \\
\hline $\mathrm{C}$ & 5.94146900 & -3.13496100 & -1.06760300 \\
\hline $\mathrm{H}$ & 4. 90292500 & -4.57243500 & 0.16559900 \\
\hline $\mathrm{H}$ & 6. 76760800 & -1.50267600 & -2.21562100 \\
\hline $\mathrm{H}$ & 6. 73830200 & -3.82389600 & -1.33248300 \\
\hline $\mathrm{C}$ & 0.13999000 & 1. 46587000 & -0.29137600 \\
\hline H & -0.35930900 & 1. 65585100 & -1.25266900 \\
\hline $\mathrm{H}$ & -0.21000600 & 2. 23773800 & 0.40275000 \\
\hline $\mathrm{Au}$ & -2.35643000 & -0.19488700 & -0.04451700 \\
\hline P & -4.69350100 & -0.45730600 & -0.31489000 \\
\hline $\mathrm{C}$ & -5.21026500 & -2.06098900 & -1.05992700 \\
\hline H & -4.87561300 & -2.88868800 & -0.42739600 \\
\hline $\mathrm{H}$ & -6.29943900 & -2.10979800 & -1.16521000 \\
\hline $\mathrm{H}$ & -4.75016100 & -2.17466800 & -2.04606500 \\
\hline $\mathrm{C}$ & -5.65097200 & -0.35860600 & 1. 25581500 \\
\hline H & -5.47787700 & 0.61105900 & 1. 73203600 \\
\hline $\mathrm{H}$ & -6.72335400 & -0.48004500 & 1. 06869600 \\
\hline H & -5.31627400 & -1.14160400 & 1.94278500 \\
\hline $\mathrm{C}$ & -5.47414000 & 0.81146000 & -1.39835700 \\
\hline $\mathrm{H}$ & -6.55339700 & 0.64372500 & -1.48082400 \\
\hline $\mathrm{H}$ & -5.29812400 & 1. 80926400 & -0.98546000 \\
\hline H & -5.02845800 & 0.76944200 & -2.39671000 \\
\hline $\mathrm{C}$ & 0.04580600 & -2.20413300 & 1. 15861100 \\
\hline H & 0.04177300 & -2.38163500 & 2. 24137100 \\
\hline H & -0.96502300 & -2.38207400 & 0.78523700 \\
\hline $\mathrm{H}$ & 0.70375600 & -2.96397700 & 0.71419400 \\
\hline
\end{tabular}




\section{INT3-Me}

\begin{tabular}{|c|c|c|c|}
\hline $\mathrm{C}$ & 1. 94883600 & 0.59504700 & -0.76701300 \\
\hline $\mathrm{C}$ & 0.10357000 & 0.45596800 & 1. 46329200 \\
\hline $\mathrm{C}$ & 2. 56857900 & -0.15011000 & 1. 64410600 \\
\hline $\mathrm{C}$ & 3. 21961000 & 0.99983400 & 2. 33088800 \\
\hline $\mathrm{C}$ & 3. 65016200 & -0.43242100 & 2. 62375900 \\
\hline N & 1. 23158800 & 1. 76560400 & -0.22635700 \\
\hline S & 1. 60803300 & 3. 37204000 & -0.86170800 \\
\hline 0 & 0.80074300 & 4. 28979000 & -0.06699000 \\
\hline 0 & 3. 06091800 & 3. 43360700 & -0.91849700 \\
\hline $\mathrm{H}$ & 1. 36030800 & 0.19254000 & -1.60218700 \\
\hline H & 2. 90832000 & 0.94521400 & -1.15682000 \\
\hline H & 3.89505600 & 1. 63174400 & 1. 75798900 \\
\hline H & 2. 66425000 & 1. 52906100 & 3. 10328700 \\
\hline $\mathrm{H}$ & 3. 36871200 & -0.85684600 & 3.58478000 \\
\hline H & 4. 62013300 & -0.76264200 & 2. 26222600 \\
\hline $\mathrm{C}$ & 0.95432000 & 3. 29647200 & -2.53673600 \\
\hline $\mathrm{H}$ & 1. 18337100 & 4. 26297500 & -2.99382400 \\
\hline $\mathrm{H}$ & 1. 45507500 & 2. 49675500 & -3.08568500 \\
\hline $\mathrm{H}$ & -0.12528300 & 3. 14349500 & -2.49073700 \\
\hline $\mathrm{C}$ & 2. 17430800 & -0.52681800 & 0.25882700 \\
\hline $\mathrm{C}$ & 1. 15762200 & -0.62768700 & 1. 41319700 \\
\hline $\mathrm{C}$ & 2. 72234800 & -1.75254200 & -0.42768700 \\
\hline $\mathrm{C}$ & 4. 10576000 & -1.96265300 & -0.51138400 \\
\hline $\mathrm{C}$ & 1. 86391300 & -2.65726800 & -1.07130500 \\
\hline $\mathrm{C}$ & 4. 61888300 & -3.06087800 & -1.20384100 \\
\hline $\mathrm{H}$ & 4. 78625600 & -1.26205300 & -0.03629600 \\
\hline $\mathrm{C}$ & 2. 37433300 & -3.75656700 & -1.76206500 \\
\hline $\mathrm{H}$ & 0.78797500 & -2.50377500 & -1.02205300 \\
\hline $\mathrm{C}$ & 3. 75436600 & -3.96162700 & -1.82813400 \\
\hline $\mathrm{H}$ & 5. 69332100 & -3.21157600 & -1.25515500 \\
\hline H & 1. 69677500 & -4.45376500 & -2.24722900 \\
\hline $\mathrm{H}$ & 4. 15306600 & -4.81784200 & -2.36434600 \\
\hline $\mathrm{C}$ & 0.34156800 & 1. 65645200 & 0.76715400 \\
\hline $\mathrm{H}$ & -0.15830000 & 2. 58061300 & 1. 04471600 \\
\hline $\mathrm{H}$ & -0.33032800 & 0.63135000 & 2. 45316700 \\
\hline $\mathrm{Au}$ & -1.79389300 & -0.02223900 & 0.42030500 \\
\hline $\mathrm{P}$ & -3.86632700 & -0.70084000 & -0.45502400 \\
\hline $\mathrm{C}$ & -4.19453700 & -2.49161600 & -0.19900000 \\
\hline $\mathrm{H}$ & -4.15775300 & -2.72621400 & 0.86884500 \\
\hline $\mathrm{H}$ & -5.18186100 & -2.75937800 & -0.59038000 \\
\hline $\mathrm{H}$ & -3.43330800 & -3.08778000 & -0.71073800 \\
\hline $\mathrm{C}$ & -5.29143500 & 0.16146300 & 0.32295500 \\
\hline $\mathrm{H}$ & -5.21629500 & 1. 23870500 & 0.14794000 \\
\hline
\end{tabular}

$$
\begin{array}{lrrr}
\text { H } & -6.23464400 & -0.20529500 & -0.09609700 \\
\text { H } & -5.28716700 & -0.01429100 & 1.40268600 \\
\text { C } & -4.07497900 & -0.43200600 & -2.26242900 \\
\text { H } & -5.05661200 & -0.79100200 & -2.59001200 \\
\text { H } & -3.99155100 & 0.63426900 & -2.49220500 \\
\text { H } & -3.29662000 & -0.96919700 & -2.81226500 \\
\text { C } & 0.74528500 & -1.97505700 & 1.97856500 \\
\text { H } & 0.39084900 & -1.85790200 & 3.01066300 \\
\text { H } & -0.06494600 & -2.43184700 & 1.39726200 \\
\text { H } & 1.58561900 & -2.67276600 & 1.99055700
\end{array}
$$

\begin{tabular}{|c|c|c|c|}
\hline $\mathrm{C}$ & 2. 31686100 & 0.48945100 & -1.16888400 \\
\hline $\mathrm{C}$ & -0.38489600 & -0.01592900 & 0.10018500 \\
\hline $\mathrm{C}$ & 1. 90106900 & -0.99364800 & 0.81635400 \\
\hline $\mathrm{C}$ & 2.51911400 & -0.19719900 & 2. 14051100 \\
\hline $\mathrm{C}$ & 2. 46286400 & -1.66352700 & 2. 07486800 \\
\hline $\mathrm{N}$ & 1. 53666400 & 1. 54633200 & -0.55356100 \\
\hline S & 2. 34441100 & 2. 73876100 & 0.31351200 \\
\hline 0 & 1. 63728500 & 2. 96400900 & 1. 57603800 \\
\hline 0 & 3. 76238600 & 2. 34294700 & 0.30306800 \\
\hline $\mathrm{H}$ & 1. 66344100 & -0.02921600 & -1.88768900 \\
\hline $\mathrm{H}$ & 3. 15292800 & 0.89283300 & -1.74176300 \\
\hline $\mathrm{H}$ & 3. 46870100 & 0.31445600 & 2. 02679300 \\
\hline $\mathrm{H}$ & 1. 72923800 & 0.38849100 & 2. 59833900 \\
\hline $\mathrm{H}$ & 1. 71629200 & -2.16369800 & 2. 68039500 \\
\hline $\mathrm{H}$ & 3. 40684800 & -2.19104100 & 2. 01561800 \\
\hline $\mathrm{C}$ & 2. 17433900 & 4. 22897900 & -0.68288100 \\
\hline $\mathrm{H}$ & 2. 68118900 & 5. 03387600 & -0.14487800 \\
\hline $\mathrm{H}$ & 2. 64327600 & 4. 06180200 & -1.65402400 \\
\hline $\mathrm{H}$ & 1. 11306400 & 4. 46059500 & -0.79338100 \\
\hline $\mathrm{C}$ & 2. 80869900 & -0.56515600 & -0.19960000 \\
\hline $\mathrm{C}$ & 0.38737800 & -0.99759200 & 0.62308900 \\
\hline $\mathrm{C}$ & 4. 13649300 & -1.11256600 & -0.39853000 \\
\hline $\mathrm{C}$ & 4. 32689700 & -2.51616600 & -0.32500800 \\
\hline $\mathrm{C}$ & 5. 22927600 & -0.29141700 & -0.77409200 \\
\hline $\mathrm{C}$ & 5.55972300 & -3.07461300 & -0.63492000 \\
\hline $\mathrm{H}$ & 3. 48661200 & -3.15924400 & -0.08521300 \\
\hline $\mathrm{C}$ & 6. 46959300 & -0.85914300 & -1.03826800 \\
\hline $\mathrm{H}$ & 5. 10875600 & 0.78610900 & -0.77975700 \\
\hline $\mathrm{C}$ & 6. 63527400 & -2.24731800 & -0.97932500 \\
\hline $\mathrm{H}$ & 5. 68667100 & -4.15235900 & -0.60994700 \\
\hline H & 7. 31038800 & -0.22115500 & -1.29168700 \\
\hline $\mathrm{H}$ & 7. 60307200 & -2.68627900 & -1.20354400 \\
\hline
\end{tabular}

\section{INT4-Me}




\begin{tabular}{lrrr} 
C & 0.08267200 & 1.34051100 & -0.38240900 \\
H & -0.39649800 & 1.55081600 & -1.34830700 \\
H & -0.27588800 & 2.10391700 & 0.31556800 \\
Au & -2.46337900 & -0.18413800 & -0.07361800 \\
P & -4.81847300 & -0.32045700 & -0.27258900 \\
C & -5.42876900 & -1.88473500 & -1.03131600 \\
H & -5.10642600 & -2.73875800 & -0.42802800 \\
H & -6.52207200 & -1.88716300 & -1.09887100 \\
H & -5.00802000 & -1.99594900 & -2.03519400 \\
C & -5.72793600 & -0.21065800 & 1.32562900 \\
H & -5.49779900 & 0.73979800 & 1.81619700 \\
H & -6.80931900 & -0.27922200 & 1.16559900 \\
H & -5.41172100 & -1.02252500 & 1.98741600 \\
$\mathrm{C}$ & -5.57227100 & 1.00197500 & -1.31022500 \\
$\mathrm{H}$ & -6.65967100 & 0.88281900 & -1.36626800 \\
$\mathrm{H}$ & -5.34158600 & 1.98305900 & -0.88436500 \\
$\mathrm{H}$ & -5.15558900 & 0.96019900 & -2.32104500 \\
$\mathrm{C}$ & -0.22682000 & -2.32474300 & 1.05951600 \\
$\mathrm{H}$ & -0.34418300 & -2.39977200 & 2.14920600 \\
$\mathrm{H}$ & -1.22097200 & -2.43714200 & 0.62223100 \\
\hline & 0.38135600 & -3.18065300 & 0.73898200 \\
\hline
\end{tabular}

\section{INT2- $\mathbf{C F}_{\mathbf{3}} \mathbf{P h}$}

\begin{tabular}{rrrr} 
C & 1.28628500 & 2.46978900 & -0.74892200 \\
C & -1.02210500 & 0.78265900 & 0.13217100 \\
C & 1.04158900 & 1.39985900 & 1.53594000 \\
C & 0.76302400 & 2.54783200 & 2.46763900 \\
C & 1.63719400 & 1.40660200 & 2.92008700 \\
N & -0.00313500 & 3.03202800 & -0.42633000 \\
S & -0.17568700 & 4.66980600 & -0.07059600 \\
O & -1.31594200 & 4.78159200 & 0.83836400 \\
O & 1.16426500 & 5.14324800 & 0.29095900 \\
H & 1.28355000 & 2.05699100 & -1.76679600 \\
H & 2.02955100 & 3.27805500 & -0.71866300 \\
H & 1.23230500 & 3.50324200 & 2.25557400 \\
H & -0.24225800 & 2.62130600 & 2.87125800 \\
H & 1.25424100 & 0.71214700 & 3.65926600 \\
H & 2.70568600 & 1.59030100 & 2.99796300 \\
$\mathrm{C}$ & -0.63662700 & 5.46296800 & -1.62359100 \\
H & -0.77143500 & 6.52646500 & -1.41138400 \\
H & 0.16616900 & 5.32216100 & -2.34993800 \\
H & -1.57419600 & 5.03120200 & -1.98005200 \\
$\mathrm{C}$ & 1.73906100 & 1.42604900 & 0.26022900 \\
$\mathrm{C}$ & -0.00163400 & 0.39949000 & 0.97461100 \\
\hline & & & \\
\hline
\end{tabular}

$$
\begin{aligned}
& \begin{array}{llll}
\text { C } & 0.14462700 & -1.03311300 & 1.37897500
\end{array} \\
& \begin{array}{llll}
\text { C } & -0.09199600 & -1.42872100 & 2.70783600
\end{array} \\
& \begin{array}{llll}
\text { C } & 0.47959900 & -2.02136800 & 0.43587700
\end{array} \\
& \begin{array}{llll}
\text { C } & 0.00247600 & -2.77038800 & 3.07990800
\end{array} \\
& \text { H } \quad-0.39467300 \quad-0.69181800 \quad 3.44567400 \\
& \begin{array}{llll}
\text { C } & 0.58850200 & -3.36112100 & 0.81173300
\end{array} \\
& \text { H } \quad 0.65757700 \quad-1.73351100 \quad-0.59621800 \\
& \begin{array}{llll}
\text { C } & 0.35218200 & -3.73895500 & 2.13543300
\end{array} \\
& \text { H } \quad-0.19911300 \quad-3.05840700 \quad 4.10765900 \\
& \text { H } \quad 0.85939600-4.10814300 \quad 0.07080100 \\
& \text { H } \quad 0.43698500 \quad-4.78113600 \quad 2.42949400 \\
& \text { C } \quad 2.85892800 \quad 0.56042400 \quad-0.06285800 \\
& \begin{array}{llll}
\text { C } & 3.34975300 & -0.38964200 & 0.86830400
\end{array} \\
& \text { C } \quad 3.53166300 \quad 0.67492100 \quad-1.30737100 \\
& \text { C } \quad 4.44501900 \quad-1.18727900 \quad 0.56523100 \\
& \text { H } \quad 2.86581000 \quad-0.50550400 \quad 1.82777500 \\
& \text { C } \quad 4.63477900 \quad-0.11213100 \quad-1.60010100 \\
& \text { H } \quad 3.20822900 \quad 1.39580900 \quad-2.04822400 \\
& \text { C } \quad 5.08980600 \quad-1.05138100 \quad-0.66698500 \\
& \text { H } \quad 4.80834500 \quad-1.90805700 \quad 1.28928600 \\
& \text { H } \quad 5.14899400 \quad 0.00128200 \quad-2.54811900 \\
& \begin{array}{llll}
\text { C } & -1.16498000 & 2.14728600 & -0.49237000
\end{array} \\
& \text { H } \quad-1.46643300 \quad 1.99512400 \quad-1.54246400 \\
& \text { H } \quad-2.00385000 \quad 2.65593300 \quad-0.00094100 \\
& \mathrm{Au} \quad-2.58759500 \quad-0.51545500 \quad-0.30264100 \\
& \text { P } \quad-4.40812600 \quad-1.94978100 \quad-0.77516600 \\
& \text { C } \quad-3.92829800 \quad-3.51587100 \quad-1.61550000 \\
& \text { H } \quad-3.21864800 \quad-4.06610000 \quad-0.99053300 \\
& \text { H } \quad-4.80592100 \quad-4.14432100 \quad-1.80111000 \\
& \text { H } \quad-3.44299300 \quad-3.28932900 \quad-2.56954200 \\
& \text { C } \quad-5.33910200 \quad-2.49336800 \quad 0.71683300 \\
& \text { H } \quad-5.73470100 \quad-1.62014200 \quad 1.24397800 \\
& \text { H } \quad-6.16987100 \quad-3.15028700 \quad 0.43775300 \\
& \text { H } \quad-4.66968700 \quad-3.02988000 \quad 1.39577600 \\
& \text { C } \quad-5.68810800 \quad-1.21106200 \quad-1.87207500 \\
& \text { H } \quad-6.50067100 \quad-1.92226800 \quad-2.05540500 \\
& \text { H } \quad-6.09925000-0.31078500 \quad-1.40579300 \\
& \text { H } \quad-5.23771900 \quad-0.92814300 \quad-2.82818100 \\
& \text { C } \quad 6.24934100-1.95793000 \quad-1.01928500 \\
& \text { F } \quad 5.80317100 \quad-3.06180600 \quad-1.65320700 \\
& \text { F } \quad 6.91279500 \quad-2.35749500 \quad 0.08029700 \\
& \text { F } \quad 7.12147500 \quad-1.34155100 \quad-1.83753100
\end{aligned}
$$

\section{TS2-CF ${ }_{3} \mathrm{Ph}$}




\begin{tabular}{|c|c|c|c|}
\hline C & 1. 50785200 & 2. 25041300 & -0.12255100 \\
\hline & -1.00336000 & 0.67972800 & -0.02908000 \\
\hline & 0.71783900 & 0.57201200 & 1. 70393200 \\
\hline & 0.06984800 & 1. 36954300 & 2. 78850000 \\
\hline & 0.82438900 & 0.09449700 & 3. 11285800 \\
\hline & 0.17066600 & 2. 87076000 & -0.08982700 \\
\hline & 0.07182100 & 4. 50696900 & -0.71334300 \\
\hline 0 & 1. 11843200 & 4. 65304800 & -1.71912000 \\
\hline & -1.34142100 & 4. 71893500 & -1.02093600 \\
\hline & 1. 85900400 & 2. 17945200 & -1.15871800 \\
\hline $\mathrm{H}$ & 2. 20627900 & 2. 90620000 & 0.40803000 \\
\hline & 0.53093000 & 2. 31702900 & 3. 05678500 \\
\hline H & -1.01304500 & 1. 35577200 & 2. 88407700 \\
\hline $\mathrm{H}$ & 0.23502000 & -0.77051200 & 3. 40371000 \\
\hline $\mathrm{H}$ & 1. 78282000 & 0.16900800 & 3. 61922900 \\
\hline c & 0.50889200 & 5. 47459900 & 0.73814500 \\
\hline & 0.50040400 & 6.52001400 & 0.41888200 \\
\hline $\mathrm{H}$ & -0.23902500 & 5. 30321700 & 1. 51344000 \\
\hline & 1. 50989400 & 5. 19816700 & 1. 07461800 \\
\hline c & 1. 53828900 & 0.85677500 & 0.51287900 \\
\hline 0 & 0.23598400 & -0.01670100 & 0.37382300 \\
\hline & 0.34359200 & -1.48211300 & 0.03629100 \\
\hline c & 0.89037600 & -2.42950900 & 0.91265700 \\
\hline c & -0.08220100 & -1.91770800 & -1.23114200 \\
\hline 0 & 0.98725800 & -3.77229100 & 0.54181600 \\
\hline H & 1. 25904400 & -2.12444400 & 1. 88392300 \\
\hline c & 0.01827400 & -3.25885200 & -1.60429300 \\
\hline & -0.48148500 & -1.20129000 & -1.94504800 \\
\hline 0 & 0.54885500 & -4.19421500 & -0.71409700 \\
\hline & 1. 41436500 & -4.48840800 & 1. 23800000 \\
\hline & -0.30794100 & -3.56757000 & -2.59358900 \\
\hline & 0.63196400 & -5.23829000 & -1.00116600 \\
\hline & 2. 88757300 & 0.21316700 & 0.30858400 \\
\hline 0 & 3.84446300 & 0.23382200 & 1. 33155400 \\
\hline & 3. 24034000 & -0.33162000 & -0.93481900 \\
\hline & 5.11838000 & -0.29434300 & 1. 12677900 \\
\hline & 3. 60049600 & 0.66910400 & 2. 29545500 \\
\hline 0 & 4. 51074600 & -0.86084800 & -1.14490300 \\
\hline & 2.51274900 & -0.36088400 & -1.74038500 \\
\hline & 5. 45171100 & -0.84459000 & -0.11197800 \\
\hline & 5.84903500 & -0.28377000 & 1. 92862400 \\
\hline $\mathrm{H}$ & 4. 76932300 & -1.29374600 & -2.10524300 \\
\hline & -0.95014700 & 2. 07550500 & -0.29174000 \\
\hline & -0.95808000 & 1. 34545500 & -1.32103200 \\
\hline
\end{tabular}

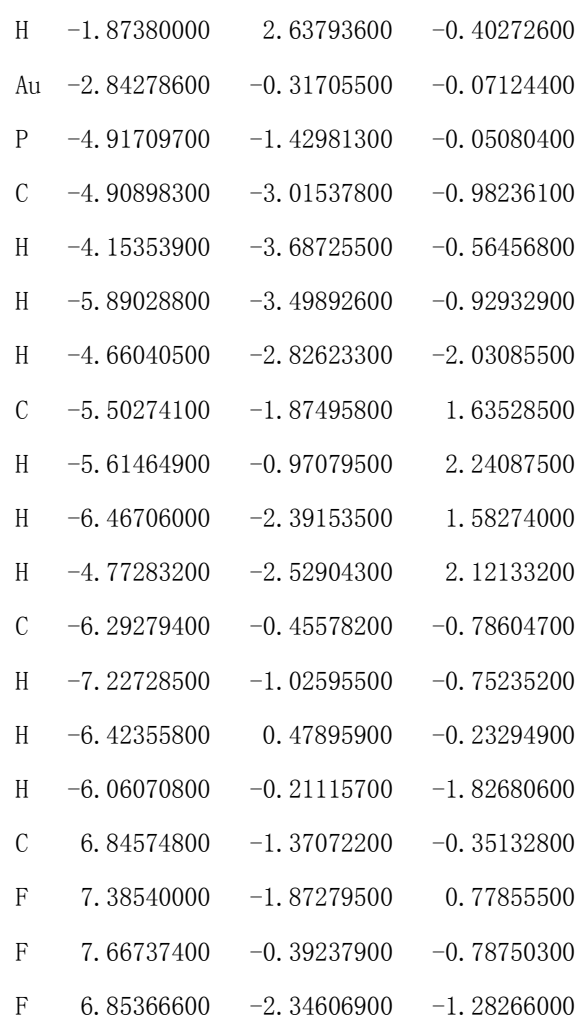

\section{TS3- $\mathrm{CF}_{3} \mathbf{P h}$}

C 1.11358500

2. 05412800

0. 01387600

C $\quad-0.81332200$

0. 05195600

$-0.99334400$

C $\quad 0.43042900$

$-0.10245000$

1. 25441500

C $\quad-0.53281600$

0. 40106800

2. 27666600

C 0.41652200

$-0.73843300$

2. 60297400

N $\quad-0.26212500$

2. 34887600

$-0.47403300$

S $\quad-0.64939300$

4. 05616600

$-0.74862800$

$0 \quad 0.59192700$

4. 69038700

$-1.16786400$

$0 \quad-1.85546800$

4. 04885200

$-1.57060400$

H 1.81093300

2. 39740500

$-0.75514600$

H 1.29235900

2. 66026500

0. 90912500

H $\quad-0.33962900$

1. 37138600

2. 73109000

H $\quad-1.59062900$

0. 16200100

2. 17009600

H $\quad-0.02102400$

$-1.72850600$

2. 69860900

H 1.23775900

$-0.55241900$

3. 28931800

C -1.07030900

4. 60681300

0. 91152000

H $\quad-1.30894500$

5. 67022100

0. 82361200

H $\quad-1.93883100$

4. 04648100

1. 26084400

H $\quad-0.20966500$

4. 47293000

1. 56918100

C 1.37385300

0. 57877600

0. 33292300

C $\quad 0.35390300$

$-0.49226800$

$-0.20227800$

C $\quad 0.87833000$

$-1.82071700$

$-0.70301300$

C $\quad 1.30106400$

$-2.83983400$

0. 16193300 


\begin{tabular}{|c|c|c|c|}
\hline C & 0.97549700 & -2.05131700 & -2.08753900 \\
\hline $\mathrm{C}$ & 1. 78484100 & -4.05121200 & -0.33673000 \\
\hline $\mathrm{H}$ & 1. 26878100 & -2.69053400 & 1. 23300500 \\
\hline 0 & 1. 45824400 & -3.26056300 & -2.58719000 \\
\hline $\mathrm{H}$ & 0.69402000 & -1.27316900 & -2.79282800 \\
\hline $\mathrm{C}$ & 1. 86131100 & -4.27025600 & -1.71170700 \\
\hline $\mathrm{H}$ & 2. 10485800 & -4.82369200 & 0.35684100 \\
\hline $\mathrm{H}$ & 1. 52532300 & -3.40869700 & -3.66127300 \\
\hline $\mathrm{H}$ & 2. 23825400 & -5.21267800 & -2.09779400 \\
\hline $\mathrm{C}$ & 2. 85619200 & 0.29754900 & 0.35164300 \\
\hline C & 3. 57279900 & 0.33785700 & 1. 55437900 \\
\hline C & 3. 55885400 & 0.08001300 & -0.84340600 \\
\hline $\mathrm{C}$ & 4. 95432400 & 0.14607000 & 1. 57068100 \\
\hline $\mathrm{H}$ & 3. 05431000 & 0.52500000 & 2. 48937900 \\
\hline $\mathrm{C}$ & 4. 93681200 & -0.11466600 & -0.83358200 \\
\hline $\mathrm{H}$ & 3. 02344900 & 0.04536300 & -1.78724900 \\
\hline $\mathrm{C}$ & 5.63710400 & -0.08405400 & 0.37605400 \\
\hline $\mathrm{H}$ & 5.49803400 & 0.16993400 & 2. 50896700 \\
\hline $\mathrm{H}$ & 5. 46773700 & -0.29912300 & -1.76159400 \\
\hline $\mathrm{C}$ & -1.00353800 & 1. 43321300 & -1.11758200 \\
\hline H & -1.08816300 & -0.50556500 & -1.89191500 \\
\hline $\mathrm{H}$ & -1.77516200 & 1. 83585100 & -1.76775900 \\
\hline $\mathrm{Au}$ & -2.84286200 & -0.40406000 & -0.13726400 \\
\hline P & -4.95320900 & -1.12439500 & 0.57456300 \\
\hline $\mathrm{C}$ & -5.94975400 & -1.85366500 & -0.78618900 \\
\hline H & -5.42739500 & -2.71817900 & -1.20616600 \\
\hline $\mathrm{H}$ & -6.92950000 & -2.17187400 & -0.41384700 \\
\hline $\mathrm{H}$ & -6.09078700 & -1.11468200 & -1.58028600 \\
\hline $\mathrm{C}$ & -4.89178800 & -2.42025800 & 1. 87651200 \\
\hline $\mathrm{H}$ & -4.38132800 & -2.03424000 & 2. 76376900 \\
\hline $\mathrm{H}$ & -5.90445900 & -2.73153400 & 2. 15432400 \\
\hline H & -4.33893800 & -3.28982300 & 1. 50905800 \\
\hline $\mathrm{C}$ & -6.00163600 & 0.21439100 & 1. 27383800 \\
\hline $\mathrm{H}$ & -6.98027400 & -0.18074200 & 1. 56714600 \\
\hline H & -5.51445300 & 0.64925900 & 2. 15147100 \\
\hline H & -6.14321100 & 1. 00227400 & 0.52825600 \\
\hline $\mathrm{C}$ & 7. 13700700 & -0.24486700 & 0.37554800 \\
\hline $\mathrm{F}$ & 7. 59023100 & -0.69907700 & 1. 56206900 \\
\hline $\mathrm{F}$ & 7. 75440500 & 0.93187200 & 0.13381600 \\
\hline $\mathrm{F}$ & 7. 53972800 & -1.10886500 & -0.57934800 \\
\hline
\end{tabular}

\section{INT3- CF F $_{3}$ Ph}

$\begin{array}{lrrr}\text { C } & 1.49066100 & 1.87781100 & -0.83327100 \\ \text { C } & -1.14342700 & 0.71967700 & 0.11556200\end{array}$

\begin{tabular}{|c|c|c|c|}
\hline C & 1. 16428400 & 0.53438700 & 1. 28201500 \\
\hline $\mathrm{C}$ & 1. 21274600 & 1. 51465400 & 2. 49516500 \\
\hline $\mathrm{C}$ & 1. 71197400 & 0.11757000 & 2. 63138300 \\
\hline $\mathrm{N}$ & 0.40344800 & 2. 69245800 & -0.35012800 \\
\hline S & 0.75969200 & 4. 16311300 & 0.39165100 \\
\hline 0 & -0.15281700 & 4. 33143300 & 1. 52268000 \\
\hline 0 & 2. 21886400 & 4. 16569400 & 0.56741500 \\
\hline $\mathrm{H}$ & 1. 17159200 & 1. 33334700 & -1.73565000 \\
\hline $\mathrm{H}$ & 2. 31646500 & 2. 51934700 & -1.14867800 \\
\hline $\mathrm{H}$ & 1. 93317900 & 2. 32533200 & 2. 45852500 \\
\hline $\mathrm{H}$ & 0.23462600 & 1. 79127000 & 2. 87445800 \\
\hline $\mathrm{H}$ & 1. 11651300 & -0.59382000 & 3. 19410400 \\
\hline $\mathrm{H}$ & 2. 78413400 & -0.01627000 & 2. 73801600 \\
\hline $\mathrm{C}$ & 0.34330600 & 5. 42049500 & -0.83084300 \\
\hline $\mathrm{H}$ & 0.54849300 & 6. 39133500 & -0.37308000 \\
\hline $\mathrm{H}$ & 0.96467000 & 5. 27898500 & -1.71697000 \\
\hline H & -0.71758500 & 5. 33881300 & -1.07602100 \\
\hline $\mathrm{C}$ & 2. 01608600 & 0.85673700 & 0.15759300 \\
\hline $\mathrm{C}$ & -0.21601800 & 0.00615800 & 0.79001800 \\
\hline $\mathrm{C}$ & -0.41508900 & -1.44959400 & 1. 10528000 \\
\hline $\mathrm{C}$ & -1.07456300 & -1.83946300 & 2. 28365000 \\
\hline $\mathrm{C}$ & 0.06088400 & -2.44993800 & 0.24184300 \\
\hline $\mathrm{C}$ & -1.26323900 & -3.19056300 & 2. 58233900 \\
\hline $\mathrm{H}$ & -1.45745100 & -1.07696600 & 2. 95717500 \\
\hline $\mathrm{C}$ & -0.12432500 & -3.80166100 & 0.54209900 \\
\hline $\mathrm{H}$ & 0.55653800 & -2.16710500 & -0.68387000 \\
\hline $\mathrm{C}$ & -0.78521100 & -4.17556800 & 1. 71459300 \\
\hline $\mathrm{H}$ & -1.78090100 & -3.47268700 & 3. 49503000 \\
\hline $\mathrm{H}$ & 0.24158500 & -4.56157700 & -0.14306500 \\
\hline H & -0.92658500 & -5.22641900 & 1.95017700 \\
\hline $\mathrm{C}$ & 3. 28102300 & 0.22122500 & -0.10142200 \\
\hline $\mathrm{C}$ & 3. 51387200 & -1.10349600 & 0.36018900 \\
\hline $\mathrm{C}$ & 4. 30494300 & 0.86529600 & -0.84808800 \\
\hline $\mathrm{C}$ & 4. 69906300 & -1.75700500 & 0.06216800 \\
\hline H & 2. 73153400 & -1.62759100 & 0.89607000 \\
\hline $\mathrm{C}$ & 5.50434200 & 0.21963100 & -1.10498300 \\
\hline $\mathrm{H}$ & 4. 18830800 & 1. 89224900 & -1.17324300 \\
\hline $\mathrm{C}$ & 5. 69965000 & -1.09303800 & -0.65846800 \\
\hline $\mathrm{H}$ & 4. 85388400 & -2.78081200 & 0.38458000 \\
\hline $\mathrm{H}$ & 6.28987500 & 0.72619000 & -1.65444400 \\
\hline $\mathrm{C}$ & -0.96870900 & 2. 14681400 & -0.35535100 \\
\hline H & -1.36566500 & 2. 22043700 & -1.37848200 \\
\hline $\mathrm{H}$ & -1.58640100 & 2. 80167400 & 0.26871900 \\
\hline $\mathrm{Au}$ & -2.99830500 & -0.11333800 & -0.34619700 \\
\hline
\end{tabular}




\begin{tabular}{lrrr} 
P & -5.10630800 & -1.04419500 & -0.86595300 \\
C & -5.03268400 & -2.50043100 & -1.99219300 \\
H & -4.42103600 & -3.28600800 & -1.53848300 \\
H & -6.03624700 & -2.89378700 & -2.18756900 \\
H & -4.57063200 & -2.21127400 & -2.94086300 \\
C & -6.04594700 & -1.65583900 & 0.59543000 \\
H & -6.22460000 & -0.83132800 & 1.29204800 \\
H & -7.00759000 & -2.08181200 & 0.28959400 \\
H & -5.46228800 & -2.42245200 & 1.11367100 \\
C & -6.27318500 & 0.11180400 & -1.69909400 \\
H & -7.22594500 & -0.38397500 & -1.91408700 \\
H & -6.45754000 & 0.97725400 & -1.05559100 \\
H & -5.83560300 & 0.46810200 & -2.63641700 \\
C & 7.02462500 & -1.78627000 & -0.90992500 \\
F & 6.85370100 & -3.11123200 & -1.06574600 \\
F & 7.85333000 & -1.59528100 & 0.13259400 \\
F & 7.62490300 & -1.30506800 & -2.01208800 \\
\hline & -50 &
\end{tabular}

\section{INT4-CF $\mathbf{3}$ Ph}

\begin{tabular}{|c|c|c|c|}
\hline $\mathrm{C}$ & 1. 59993800 & 1. 45265700 & -0.96518400 \\
\hline $\mathrm{C}$ & -1.14945700 & 0.55571400 & 0.01219600 \\
\hline $\mathrm{C}$ & 1. 17507100 & -0.31958000 & 0.77987100 \\
\hline $\mathrm{C}$ & 1. 68909000 & 0.33775600 & 2. 26240500 \\
\hline $\mathrm{C}$ & 1. 71853900 & -1.09931800 & 1.98730800 \\
\hline $\mathrm{N}$ & 0.64315800 & 2. 35455900 & -0.34790300 \\
\hline S & 1. 20339800 & 3. 43831300 & 0.81477800 \\
\hline 0 & 0.37985900 & 3. 31956300 & 2. 02153900 \\
\hline 0 & 2. 65882600 & 3. 22863700 & 0.87956900 \\
\hline $\mathrm{H}$ & 1. 08897800 & 0.96634100 & -1.81089600 \\
\hline $\mathrm{H}$ & 2. 44398800 & 2. 00280200 & -1.38331900 \\
\hline $\mathrm{H}$ & 2. 60633500 & 0.91629700 & 2. 26915900 \\
\hline $\mathrm{H}$ & 0.83135500 & 0.82282000 & 2. 71601600 \\
\hline $\mathrm{H}$ & 0.96473600 & -1.71599200 & 2. 46302100 \\
\hline $\mathrm{H}$ & 2. 69219500 & -1.56719200 & 1.91089100 \\
\hline $\mathrm{C}$ & 0.89742200 & 5. 05867100 & 0.09467600 \\
\hline $\mathrm{H}$ & 1. 23182700 & 5.80046100 & 0.82412500 \\
\hline $\mathrm{H}$ & 1. 46674300 & 5. 14511100 & -0.83213700 \\
\hline $\mathrm{H}$ & -0.17312200 & 5. 16869600 & -0.08942500 \\
\hline $\mathrm{C}$ & 2. 10367300 & 0.34225200 & -0.06561300 \\
\hline $\mathrm{C}$ & -0.32333200 & -0.39146100 & 0.52401600 \\
\hline $\mathrm{C}$ & -0.88391400 & -1.75019900 & 0.85764700 \\
\hline $\mathrm{C}$ & -1.79073900 & -1.92183400 & 1.91700800 \\
\hline $\mathrm{C}$ & -0.49195500 & -2.88205700 & 0.12414900 \\
\hline C & -2.29892500 & -3.18654400 & 2. 22502400 \\
\hline
\end{tabular}

$$
\begin{aligned}
& \text { H } \quad-2.09369500 \quad-1.05698100 \quad 2.50123800 \\
& \text { C } \quad-1.00638300 \quad-4.14452300 \quad 0.42416300 \\
& \text { H } \quad 0.20465900 \quad-2.76922400 \quad-0.70405100 \\
& \begin{array}{llll}
\text { C } & -1.91048500 & -4.30095500 & 1.47803500
\end{array} \\
& \text { H } \quad-2.99217800 \quad-3.30071100 \quad 3.05407400 \\
& \text { H } \quad-0.70187800 \quad-5.00566300 \quad-0.16433000 \\
& \text { H } \quad-2.30337200 \quad-5.28456500 \quad 1.71872900 \\
& \text { C } \quad 3.50997000 \quad-0.03717500 \quad-0.19105300 \\
& \text { C } \quad 3.84534400 \quad-1.40342400 \quad-0.33923200 \\
& \begin{array}{llll}
\text { C } & 4.53431400 & 0.93207400 & -0.28338800
\end{array} \\
& \text { C } \quad 5.15910200 \quad-1.78459000 \quad-0.57892600 \\
& \text { H } \quad 3.06414200 \quad-2.15632900 \quad-0.31793400 \\
& \begin{array}{llll}
\text { C } & 5.85249600 & 0.53981400 & -0.48519700
\end{array} \\
& \text { H } \quad 4.29635200 \quad 1.97943600 \quad-0.13481500 \\
& \text { C } \quad 6.16539400 \quad-0.81426400 \quad-0.63883700 \\
& \text { H } \quad 5.40497600 \quad-2.83051200 \quad-0.72710400 \\
& \text { H } \quad 6.63908500 \quad 1.28479400 \quad-0.53279200 \\
& \begin{array}{llll}
\text { C } & -0.78266800 & 1.96944300 & -0.36950800
\end{array} \\
& \mathrm{H} \quad-1.16027700 \quad 2.16670900 \quad-1.38179400 \\
& \text { H } \quad-1.32024600 \quad 2.65355400 \quad 0.29483600 \\
& \mathrm{Au} \quad-3.16440400 \quad 0.14354200 \quad-0.35947500 \\
& \text { P } \quad-5.43708400 \quad-0.29794800 \quad-0.82927300 \\
& \text { C } \quad-5.71879000-1.84144300 \quad-1.79478800 \\
& \text { H } \quad-5.32243700 \quad-2.69869300 \quad-1.24251000 \\
& \text { H } \quad-6.78665500-1.99728800 \quad-1.98256600 \\
& \text { H } \quad-5.19238400 \quad-1.77844200 \quad-2.75187500 \\
& \text { C } \quad-6.50335700 \quad-0.49826100 \quad 0.65932700 \\
& \text { H } \quad-6.46662500 \quad 0.41168600 \quad 1.26571600 \\
& \text { H } \quad-7.54170300 \quad-0.69625800 \quad 0.37238900 \\
& \text { H } \quad-6.13451500 \quad-1.33019600 \quad 1.26658500 \\
& \text { C } \quad-6.28321100 \quad 1.00896800 \quad-1.81372900 \\
& \text { H } \quad-7.32689200 \quad 0.73863300 \quad-2.00734400 \\
& \mathrm{H} \quad-6.25469400 \quad 1.95769100 \quad-1.26943000 \\
& \text { H } \quad-5.76588600 \quad 1.14530400 \quad-2.76809800 \\
& \text { C } \quad 7.60673900 \quad-1.24025700 \quad-0.82117200 \\
& \text { F } \quad 7.69478600 \quad-2.35342200 \quad-1.57323200 \\
& \text { F } \quad 8.33243600 \quad-0.27436800 \quad-1.41287300 \\
& \text { F } \quad 8.17234900 \quad-1.50689700 \quad 0.37233400
\end{aligned}
$$

\section{TS2-CF 3 Ph-Real}

$\begin{array}{lrrr}\text { C } & -3.65796000 & -0.72054300 & -0.65187100 \\ \text { C } & -0.70769600 & -0.48111500 & -0.44465100 \\ \text { C } & -2.24099000 & 0.16440500 & 1.34227900 \\ \text { C } & -2.05003100 & -0.97848400 & 2.28461100\end{array}$




\begin{tabular}{|c|c|c|c|c|c|c|c|}
\hline c & -2.16060300 & 0.44224600 & 2. 80533800 & C & 5. 67283700 & 1. 51326600 & 2. 90799300 \\
\hline$N$ & -2.74954200 & -1.87185800 & -0.74027200 & C & 4. 59038100 & 1. 66596100 & 3. 76835000 \\
\hline & -3.39527000 & -3.29092800 & -1.54720600 & $\mathrm{H}$ & 2. 48805200 & 1. 25178900 & 4. 03849400 \\
\hline & -4.16264300 & -2.82108600 & -2.69943100 & $\mathrm{H}$ & 6. 64426500 & 1. 90211000 & 3. 19790000 \\
\hline & -2.23829800 & -4.17544700 & -1.69257300 & $\mathrm{H}$ & 4. 71703500 & 2. 17669500 & 4. 71844600 \\
\hline & -3.90749100 & -0.36288500 & -1.65887800 & C & 6. 87315400 & 0.78305900 & 0.92451700 \\
\hline & -4.58862200 & -1.05892500 & -0.18333300 & C & 7. 40218900 & 1. 94489300 & 0.33793700 \\
\hline & -2.89277200 & -1.64864900 & 2. 43488900 & C & 7. 66515700 & -0.37397900 & 0.95178000 \\
\hline & -1.07792500 & -1.46295500 & 2. 33623000 & C & 8. 66427300 & 1. 93228700 & -0.25733700 \\
\hline & -1.25493000 & 0.90609700 & 3. 18617600 & $\mathrm{H}$ & 6. 81935300 & 2. 86202700 & 0.35049200 \\
\hline & -3.06451200 & 0.73644600 & 3. 33185200 & C & 8. 92892000 & -0.38769900 & 0.35597400 \\
\hline & -3.07163900 & 0.44078500 & 0.15678000 & $\mathrm{H}$ & 7. 30600100 & -1.25720600 & 1. 47169700 \\
\hline & -1.51223500 & 0.64276800 & 0.08258700 & C & 9. 42743200 & 0.76235500 & -0.25835700 \\
\hline & -0.94283000 & 2. 03118000 & -0.05601400 & $\mathrm{H}$ & 9. 05397800 & 2. 83815700 & -0.71302900 \\
\hline & -1.05238200 & 3. 00424500 & 0.94701100 & $\mathrm{H}$ & 9. 52954900 & -1.29238300 & 0.38871000 \\
\hline & -0.31742200 & 2. 38990800 & -1.26257800 & $\mathrm{H}$ & 10. 41158900 & 0.75273000 & -0.71760700 \\
\hline & -0.53164300 & 4. 28607700 & 0.75859600 & C & 4. 36414200 & 0.37191800 & -1.89948500 \\
\hline & -1.55971300 & 2. 77393100 & 1.87571800 & C & 4. 32307000 & -2.39947600 & -0.20269500 \\
\hline & 0.19937100 & 3. 67199400 & -1.45376700 & C & 3. 25453600 & -3.25103700 & -0.92509500 \\
\hline & -0.24162100 & 1. 66448600 & -2.06859800 & $\mathrm{H}$ & 2. 27277400 & -3.17015900 & -0.44709300 \\
\hline & 0.09964000 & 4. 62509700 & -0.43865300 & $\mathrm{H}$ & 3. 14370000 & -2.97933500 & -1.97963800 \\
\hline & -0.62771800 & 5. 02291900 & 1. 55100500 & $\mathrm{H}$ & 3. 56114100 & -4.30387700 & -0.88803400 \\
\hline & 0.67063200 & 3. 92637500 & -2.39910600 & C & 5. 69123300 & -2.72224200 & -0.83229000 \\
\hline & 0.49885800 & 5. 62456600 & -0.58408600 & $\mathrm{H}$ & 5.96952700 & -3.74603300 & -0.55191200 \\
\hline & -3.98351700 & 1. 64073000 & 0.09235400 & $\mathrm{H}$ & 5. 64997300 & -2.68980800 & -1.92433000 \\
\hline & -4.88454400 & 1. 89968100 & 1. 13336900 & $\mathrm{H}$ & 6. 48843400 & -2.05698300 & -0.49939800 \\
\hline & -4.02026400 & 2. 45473800 & -1.04926000 & C & 4. 32958200 & -2.78849400 & 1. 29029000 \\
\hline & -5.78947800 & 2. 95689200 & 1. 04767900 & $\mathrm{H}$ & 5. 11738600 & -2.27797200 & 1. 85104800 \\
\hline & -4.88738000 & 1. 26943100 & 2. 01708100 & $\mathrm{H}$ & 3. 37358100 & -2.57033000 & 1. 77930000 \\
\hline & -4.92094500 & 3. 51254000 & -1.14026700 & $\mathrm{H}$ & 4. 50380300 & -3.86840800 & 1. 36993000 \\
\hline & -3.32907400 & 2. 27353500 & -1.86683800 & C & 3. 48335100 & -0.13473800 & -3.06479200 \\
\hline & -5.80648600 & 3. 76626700 & -0.08946000 & $\mathrm{H}$ & 3. 76133800 & 0.41369200 & -3.97332100 \\
\hline & -6.47623700 & 3. 15523600 & 1. 86374700 & $\mathrm{H}$ & 3. 63607300 & -1.19943500 & -3.26949400 \\
\hline & -4.93140600 & 4. 14532100 & -2.02125700 & $\mathrm{H}$ & 2. 41594600 & 0.03274000 & -2.88562500 \\
\hline & -1.38645300 & -1.65314700 & -0.88171000 & C & 4. 05692700 & 1. 86457300 & -1.65057700 \\
\hline & -1.02047600 & -0.88911500 & -1.81317100 & $\mathrm{H}$ & 3. 01523500 & 2. 03137300 & -1.35374800 \\
\hline & -0.81846200 & -2.55811300 & -1.08316900 & $\mathrm{H}$ & 4. 70478500 & 2. 29245400 & -0.88105000 \\
\hline & 1. 38290800 & -0.48156200 & -0.39359100 & $\mathrm{H}$ & 4. 23195500 & 2. 41835000 & -2.58122000 \\
\hline & 3. 79244900 & -0.54569400 & -0.30637100 & C & 5. 83580100 & 0. 20730300 & -2.32586900 \\
\hline & 4. 30839000 & 0.34426300 & 1. 26546900 & $\mathrm{H}$ & 6.54437200 & 0.42983500 & -1.53161900 \\
\hline & 3. 22799800 & 0.51488900 & 2. 16016800 & $\mathrm{H}$ & 6. 04175500 & -0.79397100 & -2.70780600 \\
\hline & 5. 57444000 & 0.85376700 & 1. 66800300 & $\mathrm{H}$ & 6. 02803800 & 0.91035100 & -3.14621300 \\
\hline & 3. 35434700 & 1. 15258200 & 3. 39092300 & C & -4.51266400 & -3.93962000 & -0.32549400 \\
\hline & 2. 24743900 & 0.14231400 & 1. 88086000 & C & -3.99916600 & -4.67032400 & 0.75176200 \\
\hline
\end{tabular}




$\begin{array}{lrrr}\text { C } & -5.88674800 & -3.74422100 & -0.48510400 \\ \text { C } & -4.88500900 & -5.19684300 & 1.68591700 \\ \text { H } & -2.93101600 & -4.83785900 & 0.84276300 \\ \text { C } & -6.75498600 & -4.28189700 & 0.46404300 \\ \text { H } & -6.26396700 & -3.19957700 & -1.34401300 \\ \text { C } & -6.27317500 & -5.01420500 & 1.55864700 \\ \text { H } & -4.49639300 & -5.76995500 & 2.52347900 \\ \text { H } & -7.82528600 & -4.13746100 & 0.34546200 \\ \text { C } & -7.22281100 & -5.62475600 & 2.55927200 \\ \text { H } & -8.17164200 & -5.08138800 & 2.59695200 \\ \text { H } & -7.45065600 & -6.66422500 & 2.28967100 \\ \text { H } & -6.79239100 & -5.63774900 & 3.56570800 \\ \text { C } & -6.81991500 & 4.87718300 & -0.20761400 \\ \text { F } & -7.13293000 & 5.39319600 & 0.99941600 \\ \text { F } & -7.96897300 & 4.43321600 & -0.76203300 \\ \text { F } & -6.36566900 & 5.88365300 & -0.98225200\end{array}$

\section{TS3-CF 3 Ph-Real}

\begin{tabular}{|c|c|c|c|}
\hline $\mathrm{C}$ & 3. 57014900 & 0.84883600 & -0.24990000 \\
\hline $\mathrm{C}$ & 0.70415400 & 0.27560200 & 0.54015500 \\
\hline $\mathrm{C}$ & 2. 85625700 & -0.82470700 & 1. 48387900 \\
\hline $\mathrm{C}$ & 3. 16138800 & -0.19954700 & 2. 88100700 \\
\hline $\mathrm{C}$ & 3. 23326700 & -1.67504500 & 2. 67838900 \\
\hline $\mathrm{N}$ & 2.75740400 & 1. 75973400 & 0.51421200 \\
\hline S & 3.50755100 & 2. 97623400 & 1. 41215400 \\
\hline 0 & 2. 65503600 & 3. 20346700 & 2. 57993200 \\
\hline 0 & 4. 90356100 & 2.52935400 & 1. 53469700 \\
\hline $\mathrm{H}$ & 3. 10281100 & 0.64595400 & -1.22717600 \\
\hline $\mathrm{H}$ & 4. 53697300 & 1. 30544900 & -0.47171100 \\
\hline $\mathrm{H}$ & 4. 08469500 & 0.36101700 & 2. 99057100 \\
\hline $\mathrm{H}$ & 2.29605700 & 0.25084900 & 3. 35615900 \\
\hline $\mathrm{H}$ & 2. 44329200 & -2.29236500 & 3. 09401500 \\
\hline $\mathrm{H}$ & 4. 21741500 & -2.13315100 & 2. 70485200 \\
\hline $\mathrm{C}$ & 3. 79084200 & -0.48474700 & 0.43623300 \\
\hline $\mathrm{C}$ & 1. 38713300 & -0.81106600 & 0.95852000 \\
\hline C & 0.79711200 & -2.19213400 & 0.91640900 \\
\hline $\mathrm{C}$ & -0.00932600 & -2.65831400 & 1.96788000 \\
\hline $\mathrm{C}$ & 1. 05554400 & -3.05430400 & -0.16143100 \\
\hline $\mathrm{C}$ & -0.55197000 & -3.94477600 & 1.93513200 \\
\hline $\mathrm{H}$ & -0.22433900 & -1.99954000 & 2. 80512900 \\
\hline $\mathrm{C}$ & 0.51763000 & -4.34311400 & -0.19288300 \\
\hline $\mathrm{H}$ & 1. 66169900 & -2.70301900 & -0.99331700 \\
\hline $\mathrm{C}$ & -0.28643600 & -4.79262700 & 0.85712600 \\
\hline $\mathrm{H}$ & -1.18052600 & -4.28437300 & 2. 75372800 \\
\hline
\end{tabular}

\begin{tabular}{|c|c|c|c|}
\hline $\mathrm{H}$ & 0.71988900 & -4.99204000 & -1.04078500 \\
\hline $\mathrm{H}$ & -0.70396700 & -5.79509400 & 0.83475700 \\
\hline $\mathrm{C}$ & 4. 85486300 & -1.34624600 & -0.01009600 \\
\hline $\mathrm{C}$ & 4. 72604200 & -2.75671400 & 0.11420600 \\
\hline C & 6. 03663900 & -0.82549600 & -0.60108000 \\
\hline C & 5. 71994500 & -3.59948000 & -0.35595000 \\
\hline $\mathrm{H}$ & 3.81525300 & -3.17500200 & 0.52598000 \\
\hline $\mathrm{C}$ & 7. 04598500 & -1.67494800 & -1.02988300 \\
\hline $\mathrm{H}$ & 6.19423000 & 0.24447500 & -0.66622900 \\
\hline $\mathrm{C}$ & 6.88571700 & -3.06014100 & -0.91580300 \\
\hline H & 5.59881000 & -4.67551100 & -0.29140800 \\
\hline H & 7. 95680300 & -1.26992200 & -1.45581500 \\
\hline $\mathrm{C}$ & 1. 29168900 & 1. 66513100 & 0.41565700 \\
\hline $\mathrm{H}$ & 0.96824600 & 2. 09113900 & -0.54669100 \\
\hline $\mathrm{H}$ & 0.86677000 & 2. 29987600 & 1. 19990800 \\
\hline $\mathrm{Au}$ & -1.31324100 & 0.17748600 & 0.01991200 \\
\hline P & -3.65752200 & 0.11555800 & -0.60224400 \\
\hline $\mathrm{C}$ & -4.65180400 & -0.15904700 & 0.97376000 \\
\hline $\mathrm{C}$ & -3.87153900 & 0.08582600 & 2. 12678300 \\
\hline $\mathrm{C}$ & -6.00551100 & -0.54824900 & 1. 17856400 \\
\hline $\mathrm{C}$ & -4.36705900 & -0.03346400 & 3. 42227000 \\
\hline H & -2.83258300 & 0.37587300 & 2. 00590300 \\
\hline $\mathrm{C}$ & -6.47925300 & -0.68057100 & 2. 49801500 \\
\hline $\mathrm{C}$ & -5.68681100 & -0.42753000 & 3. 61304700 \\
\hline $\mathrm{H}$ & -3.71834100 & 0.17532000 & 4. 26819700 \\
\hline $\mathrm{H}$ & -7.51294100 & -0.98336300 & 2. 63590500 \\
\hline $\mathrm{H}$ & -6.09986800 & -0.53651400 & 4. 61173600 \\
\hline $\mathrm{C}$ & -7.04987100 & -0.85094600 & 0.14575000 \\
\hline $\mathrm{C}$ & -7.39909200 & -2.18290400 & -0.13068800 \\
\hline $\mathrm{C}$ & -7.82626200 & 0.17346400 & -0.41562000 \\
\hline $\mathrm{C}$ & -8.45352500 & -2.48022700 & -0.99536800 \\
\hline $\mathrm{H}$ & -6.84314400 & -2.98934800 & 0.33956600 \\
\hline $\mathrm{C}$ & -8.88082100 & -0.12227700 & -1.28305000 \\
\hline $\mathrm{H}$ & -7.62303600 & 1. 20611000 & -0.14683300 \\
\hline $\mathrm{C}$ & -9.19070800 & -1.44983700 & -1.58303600 \\
\hline $\mathrm{H}$ & -8.70406800 & -3.51688600 & -1.20257000 \\
\hline $\mathrm{H}$ & -9.46975200 & 0.68514400 & -1.70920100 \\
\hline $\mathrm{H}$ & -10.01325900 & -1.68040500 & -2.25386800 \\
\hline $\mathrm{C}$ & -3.84054700 & -1.33430000 & -1.84866300 \\
\hline $\mathrm{C}$ & -4.11319100 & 1. 84747900 & -1.32014800 \\
\hline $\mathrm{C}$ & -2.89568400 & 2. 38197000 & -2.10732900 \\
\hline $\mathrm{H}$ & -2.00274700 & 2. 46310100 & -1.47918900 \\
\hline $\mathrm{H}$ & -2.64830400 & 1. 75528400 & -2.96984500 \\
\hline $\mathrm{H}$ & -3.13680200 & 3. 38266200 & -2.48855800 \\
\hline
\end{tabular}




\begin{tabular}{lrrr} 
C & -5.33668700 & 1.88722800 & -2.25444300 \\
H & -5.61545700 & 2.93591700 & -2.41942900 \\
H & -5.11245400 & 1.45784600 & -3.23425100 \\
H & -6.20723900 & 1.37097200 & -1.84942300 \\
C & -4.35680000 & 2.77452600 & -0.11020700 \\
H & -5.25753500 & 2.50197700 & 0.44743800 \\
H & -3.51300100 & 2.77104700 & 0.58902500 \\
H & -4.48297800 & 3.80179600 & -0.47404200 \\
C & -2.63820000 & -1.26609600 & -2.81905000 \\
H & -2.68782500 & -2.13081700 & -3.49301000 \\
H & -2.65993600 & -0.36704600 & -3.44321700 \\
H & -1.67722100 & -1.29844200 & -2.29579500 \\
C & -3.71920600 & -2.62521400 & -1.00932700 \\
F & 8.83958400 & -3.39718800 & -2.20833400 \\
H & -2.80544900 & -2.64037900 & -0.40520200 \\
H & -4.57378100 & -2.76375800 & -0.34250500 \\
H & 3.957871200 & -5.08834700 & -1.95534400 \\
H & -3.68293700 & -3.48500100 & -1.68995600 \\
C & -5.12210300 & -1.37901200 & -2.70310100 \\
H & -6.03716400 & -1.29829400 & -2.12057100 \\
H & -5.12578700 & -0.59899100 & -3.46726600 \\
H & -5.14851600 & -2.34275500 & -3.22766800 \\
C & 3.48433900 & 4.46090500 & 0.41705400 \\
C & 2.38052100 & 5.31562700 & 0.48451200 \\
C & 4.56322900 & 4.74427500 & -0.42502300 \\
C & 2.35872000 & 6.45560200 & -0.31606800 \\
H & 1.56765200 & 5.10477100 & 1.17114000 \\
C & 4.52212900 & 5.89026200 & -1.21559400 \\
H & 5.43136500 & 4.09403300 & -0.43625500 \\
\hline
\end{tabular}




\section{References}

(1) Kang, R.; Lai, W.; Yao, J.; Shaik, S.; Chen, H. How Accurate Can a Local Coupled Cluster Approach Be in Computing the Activation Energies of Late-Transition-Metal-Catalyzed Reactions with Au, Pt, and Ir? J. Chem. Theory Comput. 2012, 8, 3119.

(2) Hehre, W. J.; Radom, L.; Schleyer, P. v. R.; Pople. J. A. Ab Initio Molecular Orbital Theory; Wiley: New York, 1986.

(3) Zhao, Y.; Truhlar, D. G. A New Local Density Functional for Main-Group Thermochemistry, Transition Metal Bonding, Thermochemical Kinetics, and Noncovalent Interactions. J. Chem. Phys. 2006, 125, 194101.

(4) Zhao, Y.; Truhlar, D. G. The M06 Suite of Density Functionals for Main Group Thermochemistry, Thermochemical Kinetics, Noncovalent Interactions, Excited States, and Transition Elements: Two New Functionals and Systematic Testing of Four M06-Class Functionals and 12 Other Functionals. Theor. Chem. Acc. 2008, 120, 215.

(5) Grimme, S.; Antony, J.; Ehrlich, S.; Krieg, H. A Consistent and Accurate Ab Initio Parametrization of Density Functional Dispersion Correction (DFT-D) for the 94 Elements H-Pu. J. Chem. Phys. 2010, 132, 154104.

(6) Boese, A. D.; Martin, J. M. L. Development of Density Functionals for Thermochemical Kinetics. J. Chem. Phys. 2004, 121, 3405. 
VI. NMR Spectra 

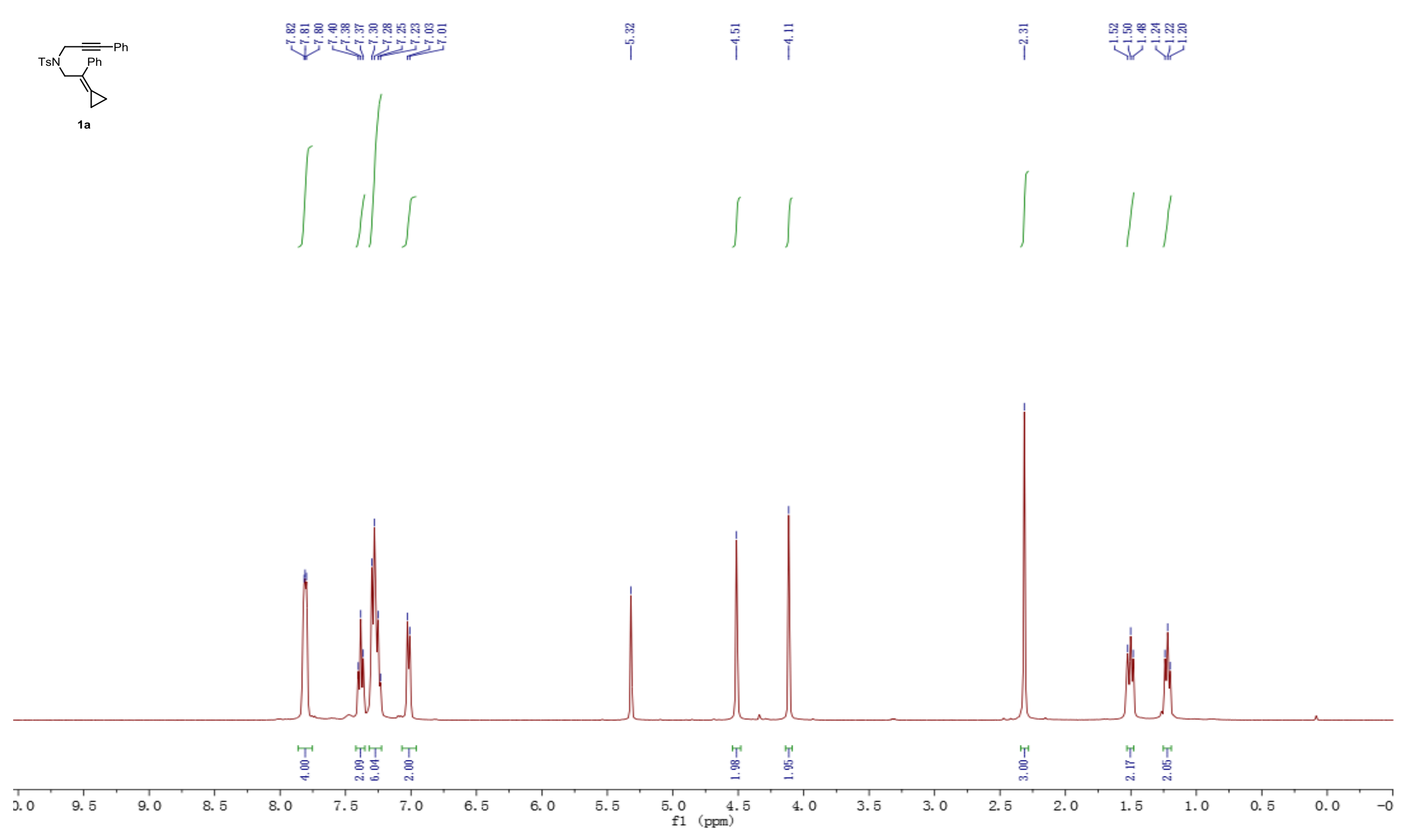

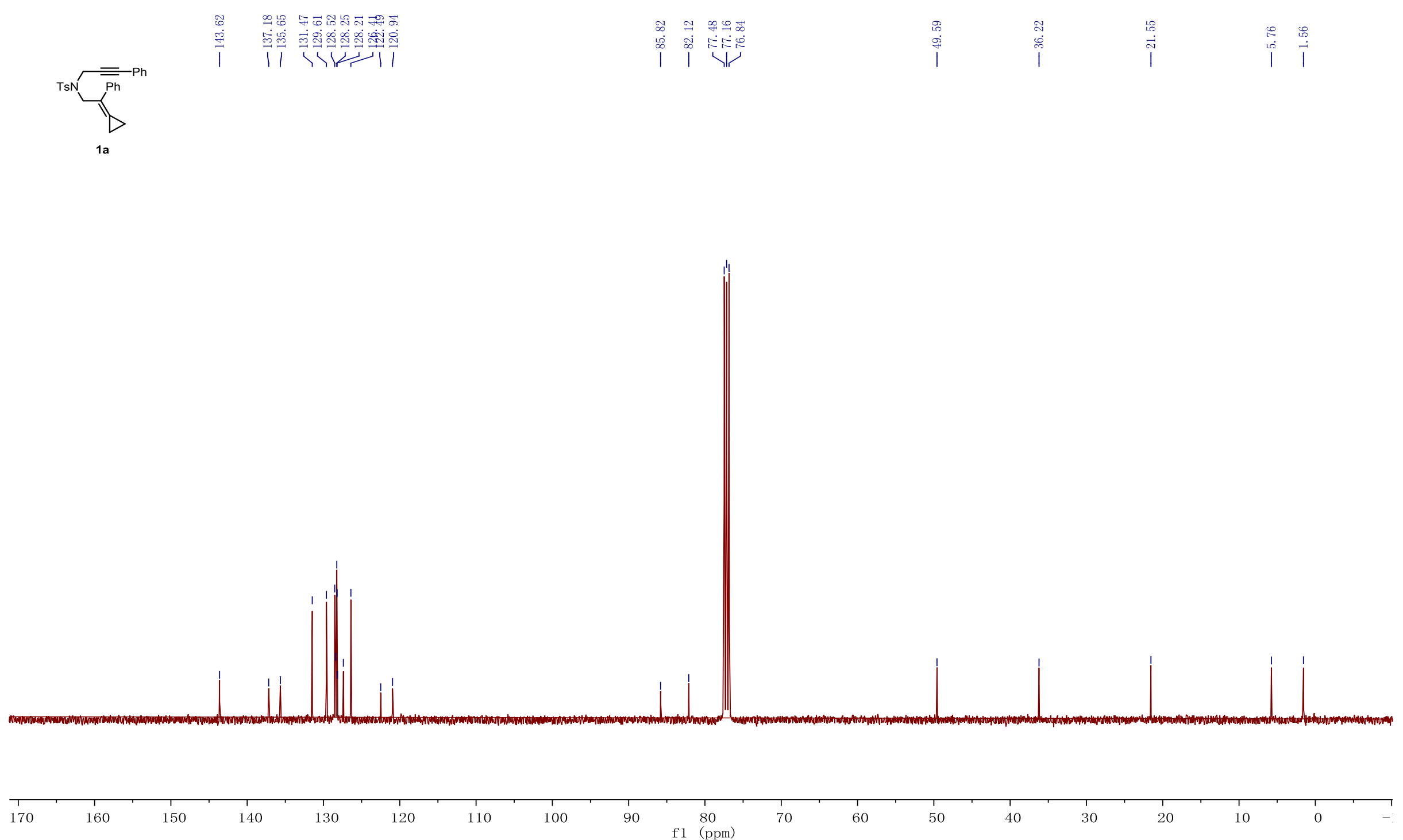


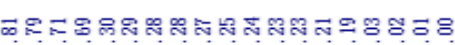
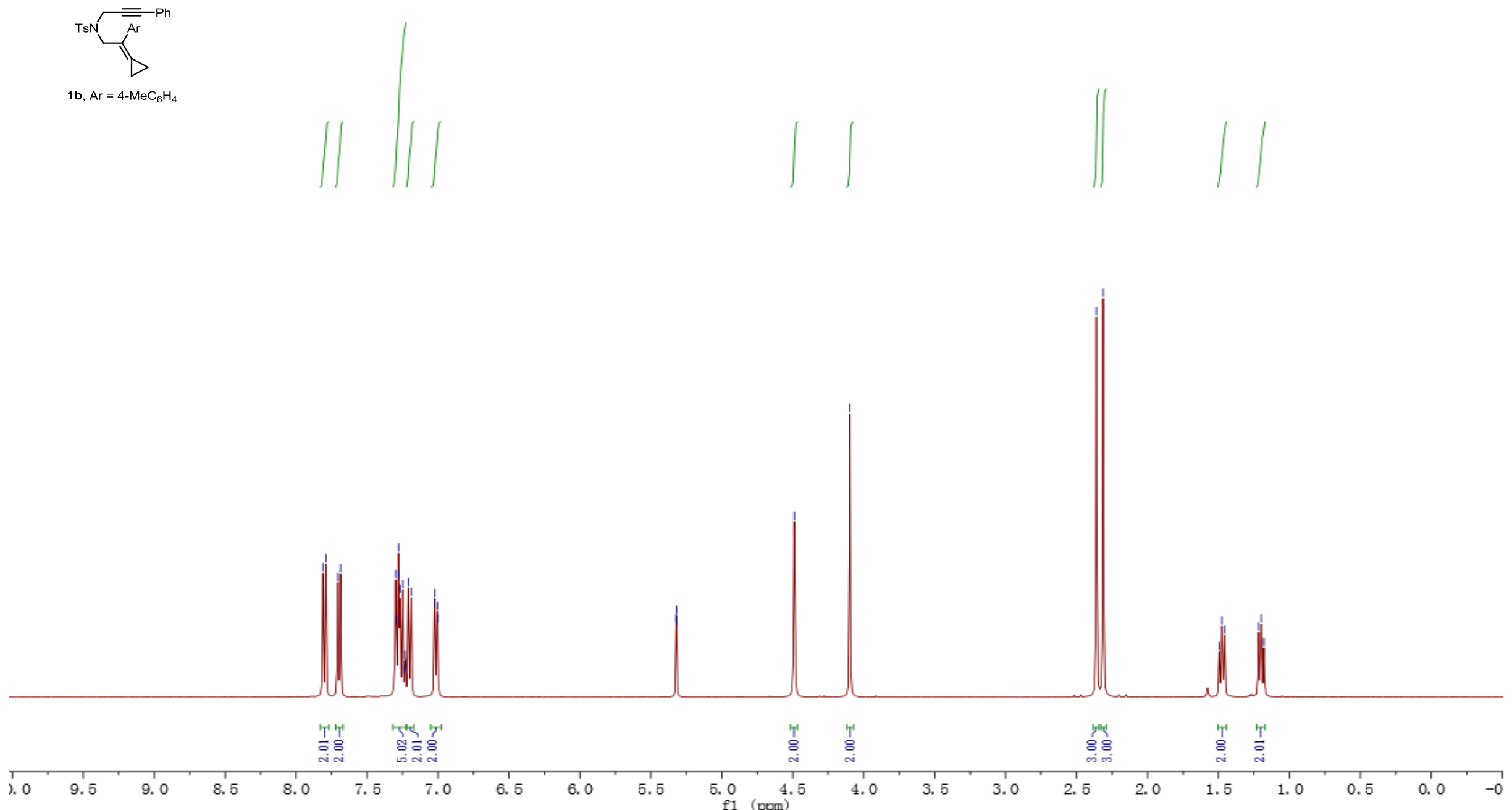


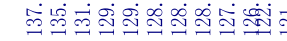

$\sqrt{\underbrace{\mathrm{Ar}}}$

$\begin{array}{ll}\infty & \overrightarrow{0} \\ \infty & \overrightarrow{0} \\ 1 & 0 \\ 1 & 0 \\ 1 & 1\end{array}$

$\infty=1500$

苗

$\infty$
$\infty$
$\infty$
$\infty$
$\infty$
1

1b, $\mathrm{Ar}=4-\mathrm{MeC}_{6} \mathrm{H}_{4}$

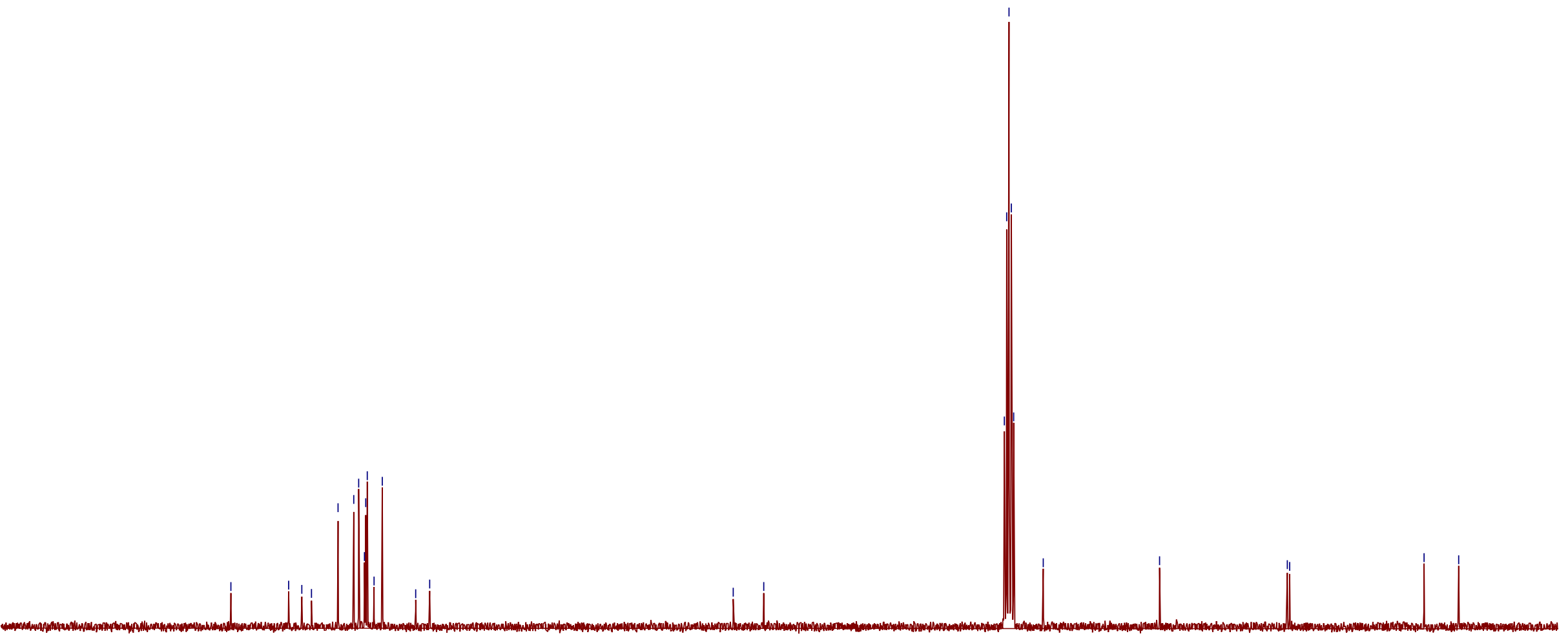




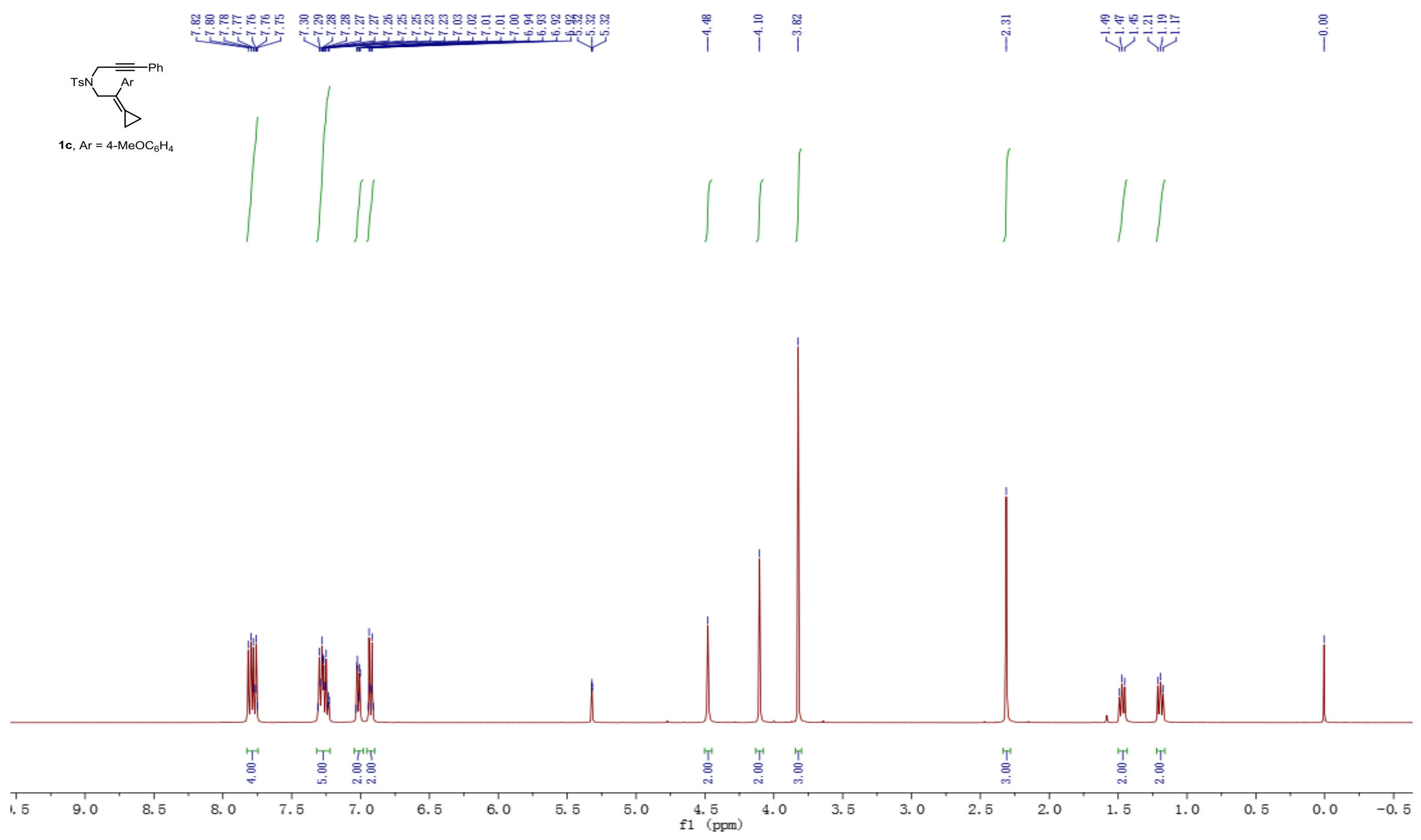




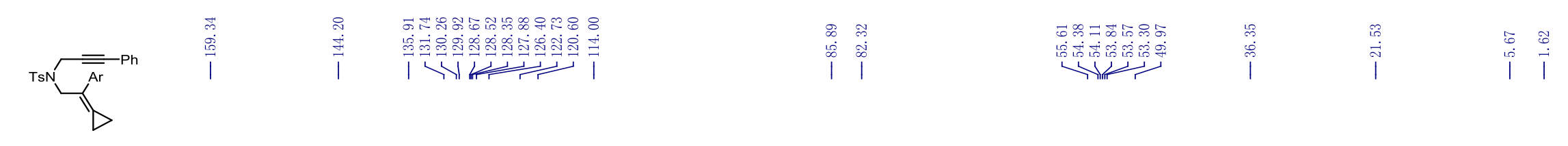

1c, $\mathrm{Ar}=4-\mathrm{MeOC}_{6} \mathrm{H}_{4}$

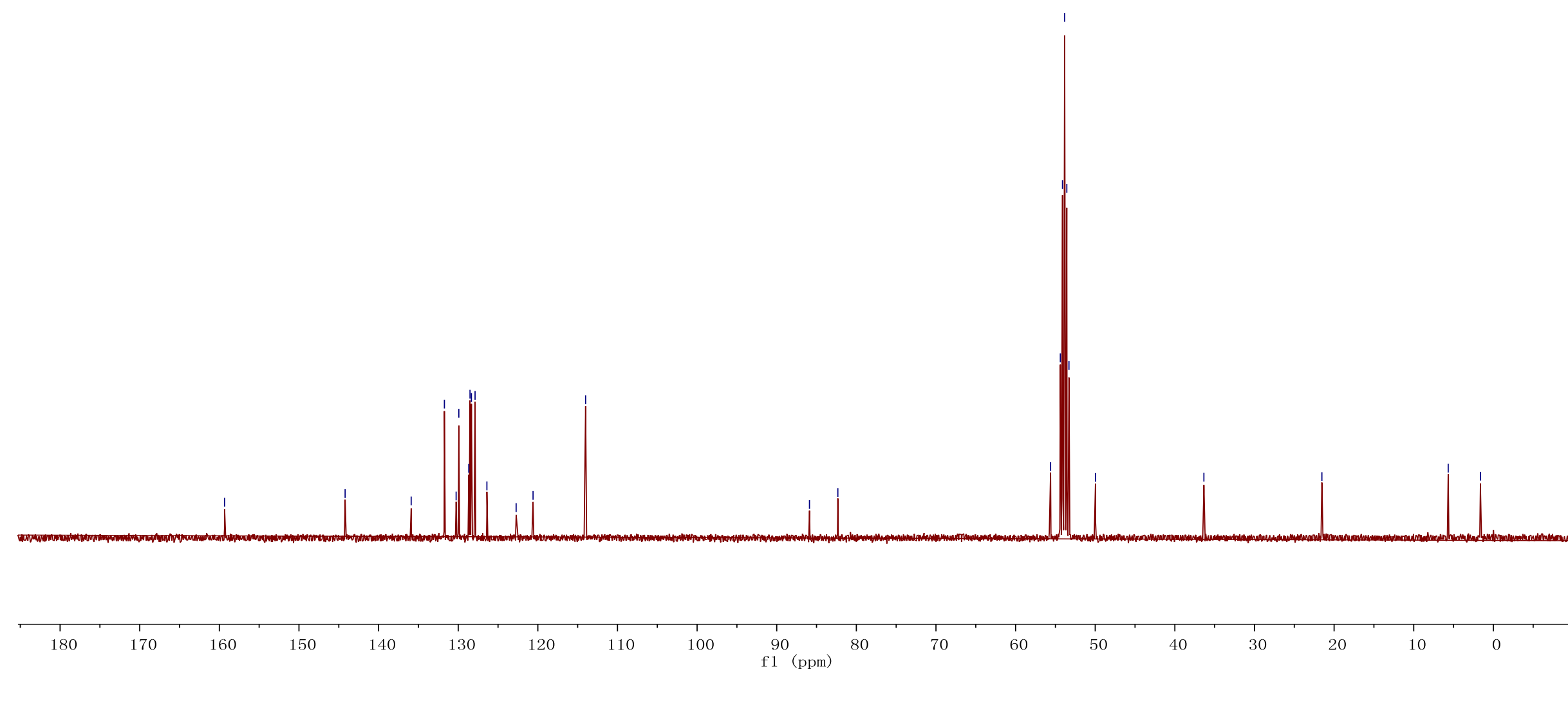




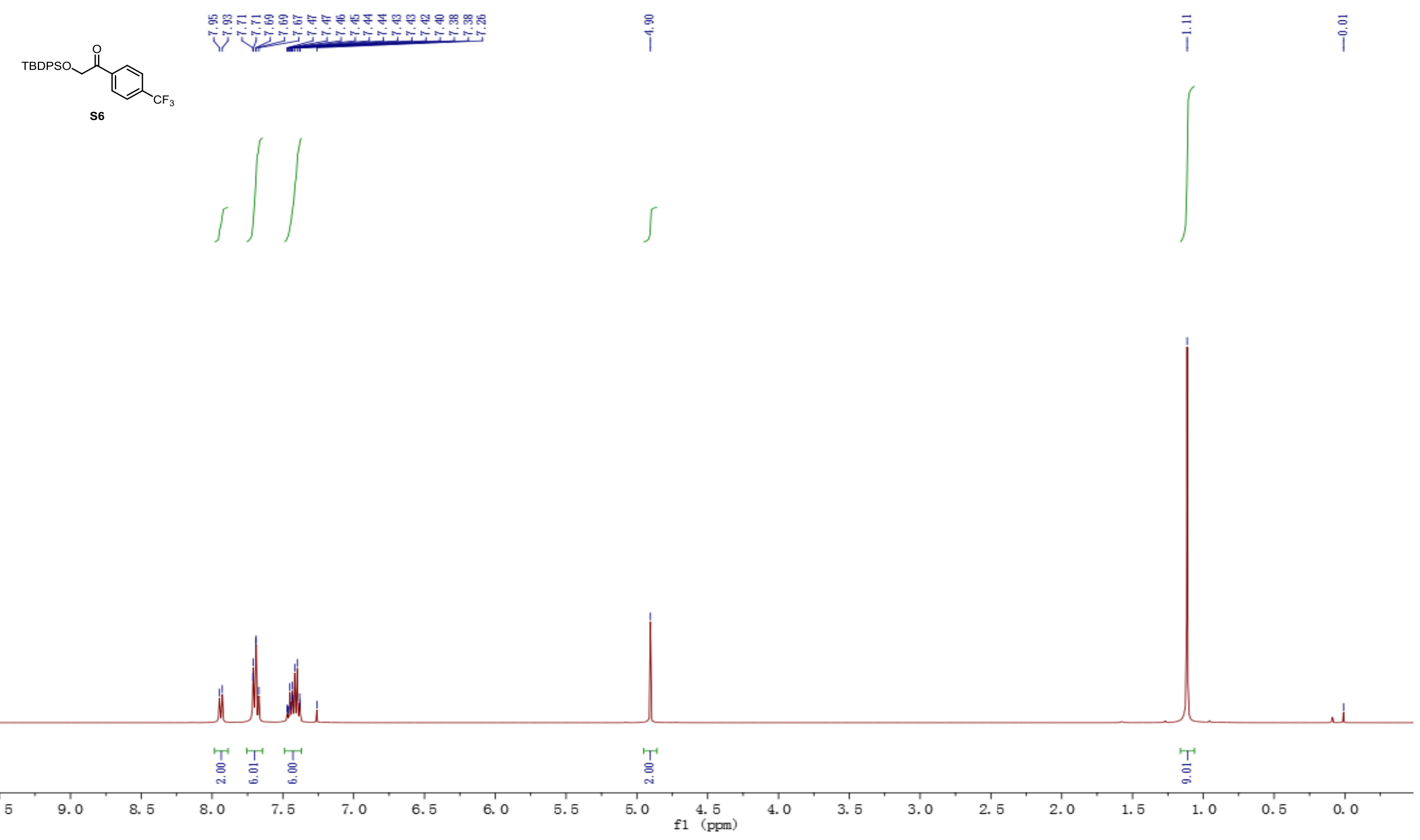



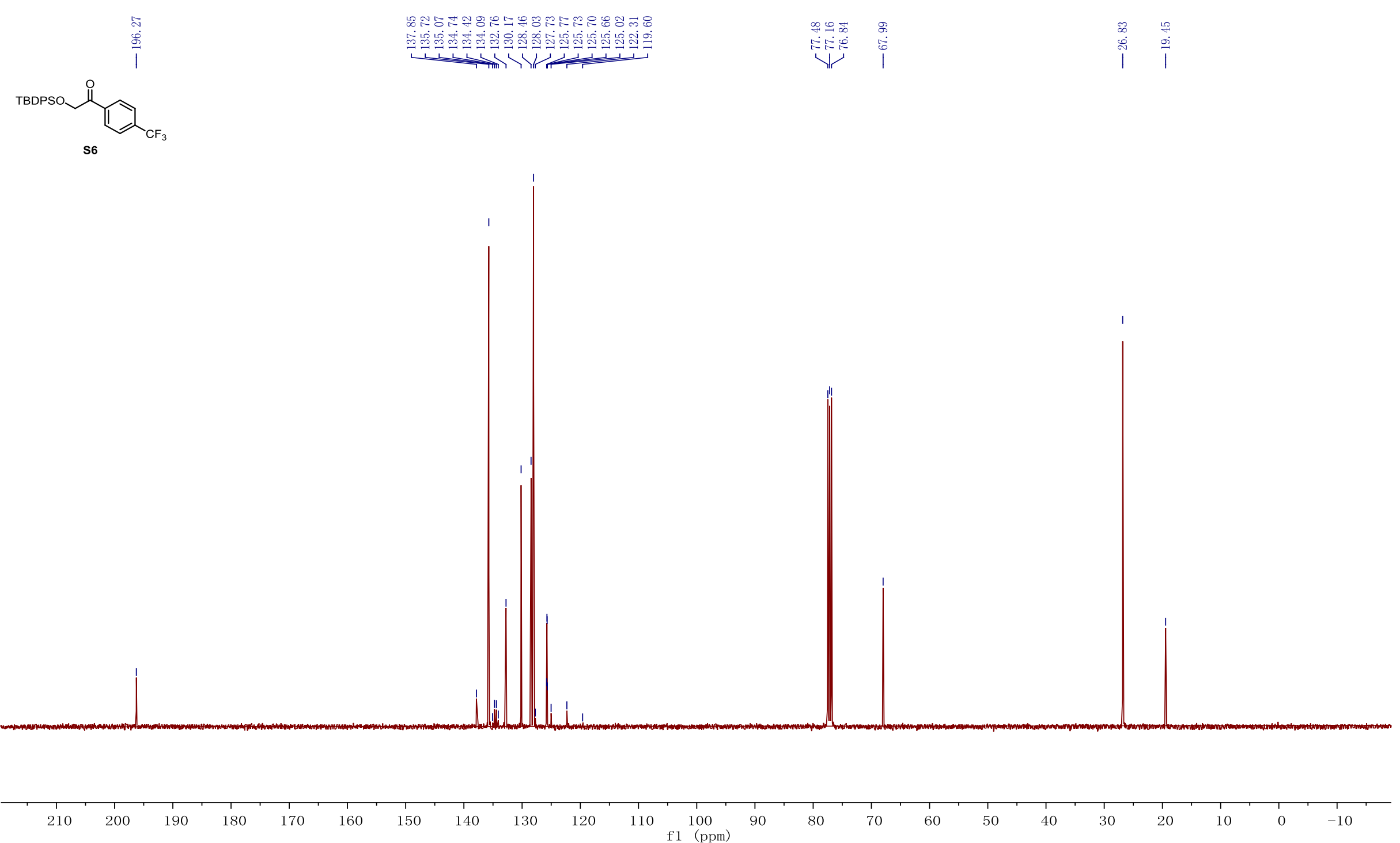

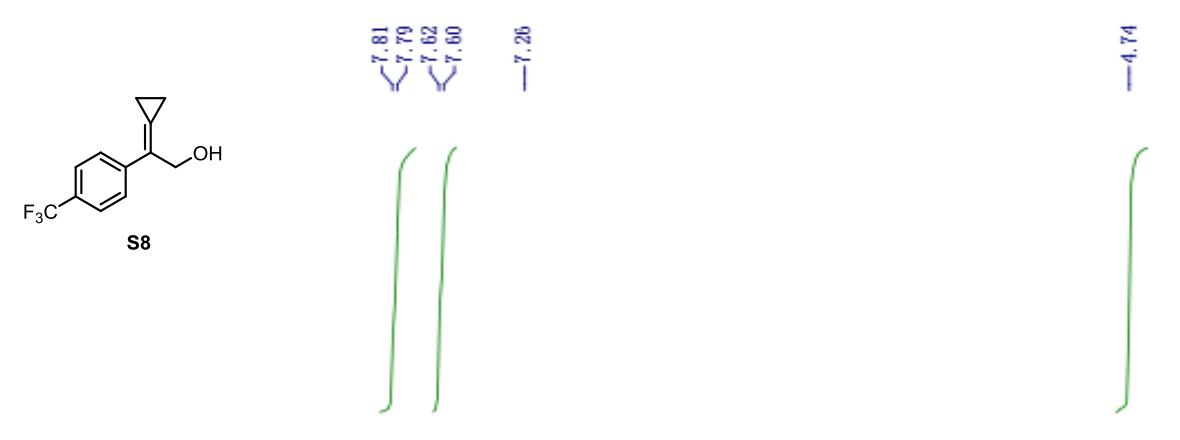

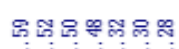

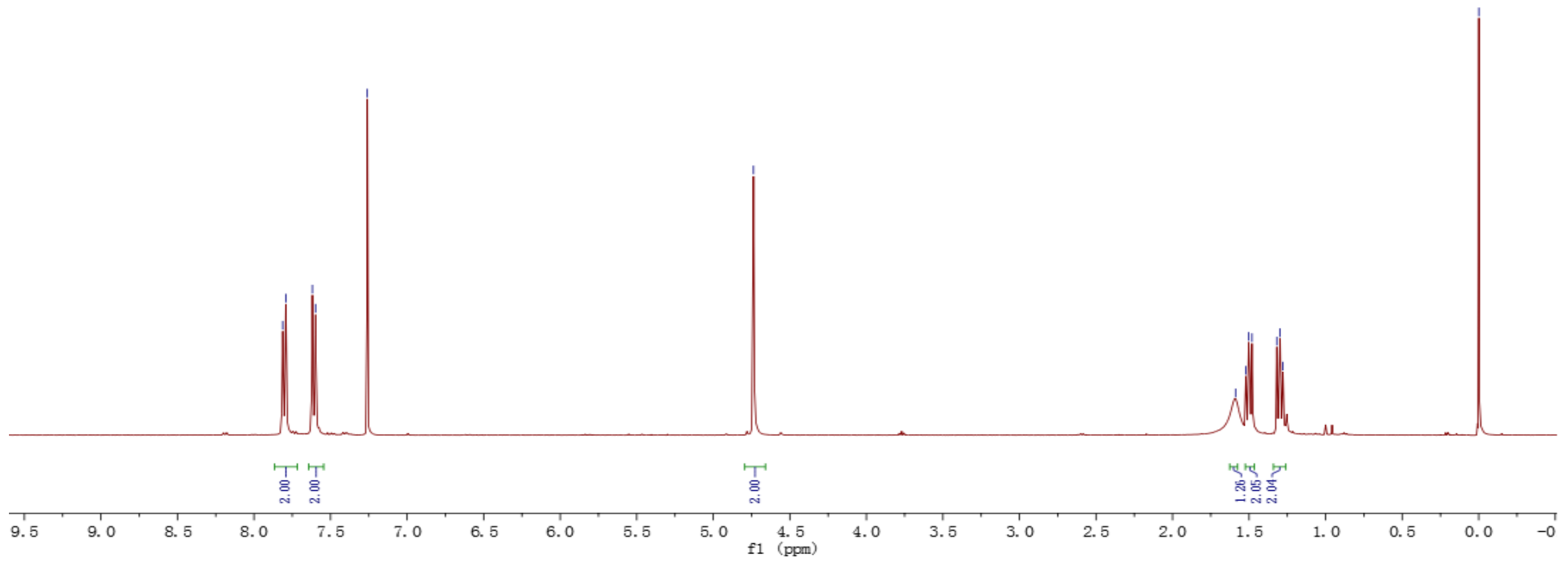




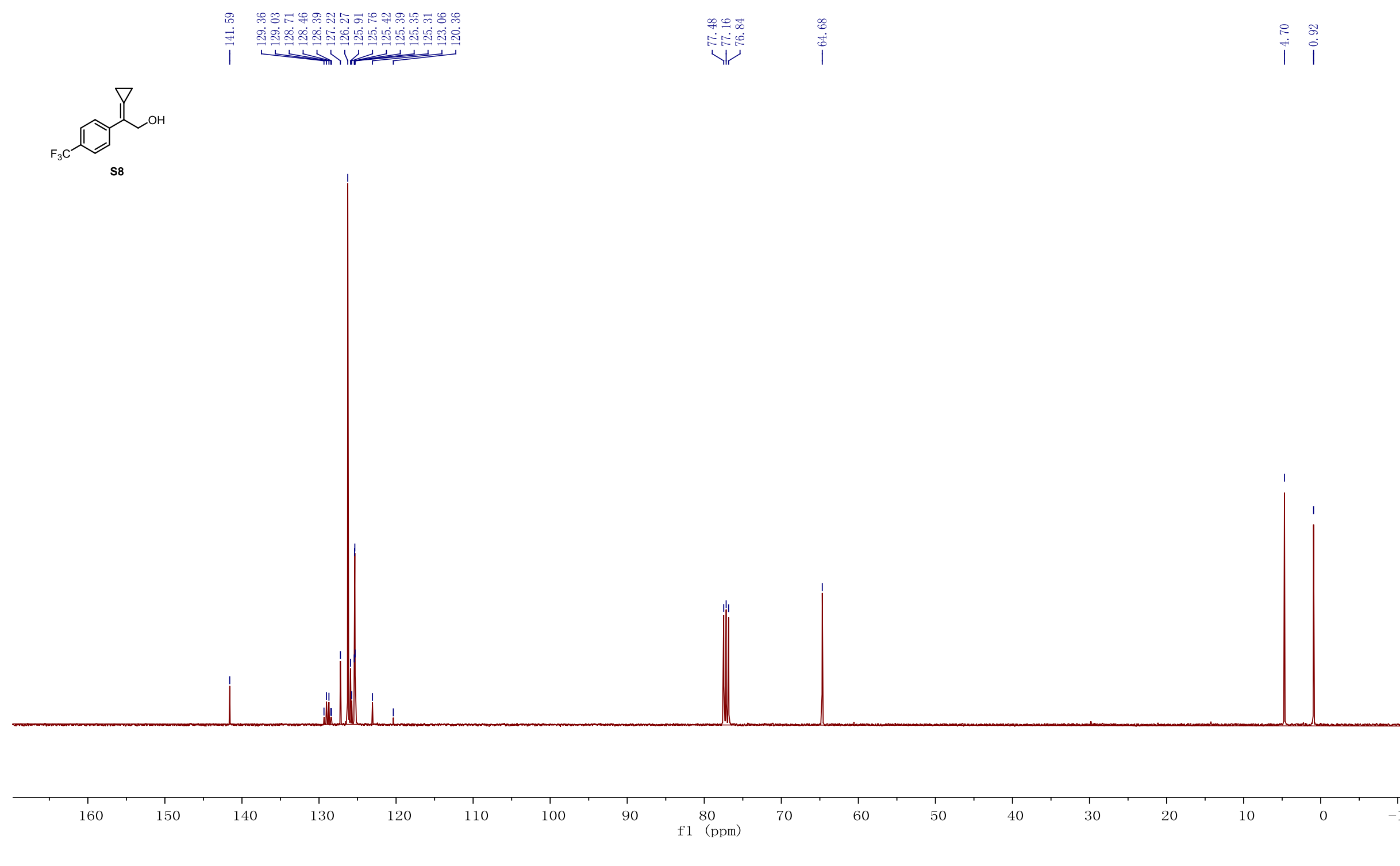




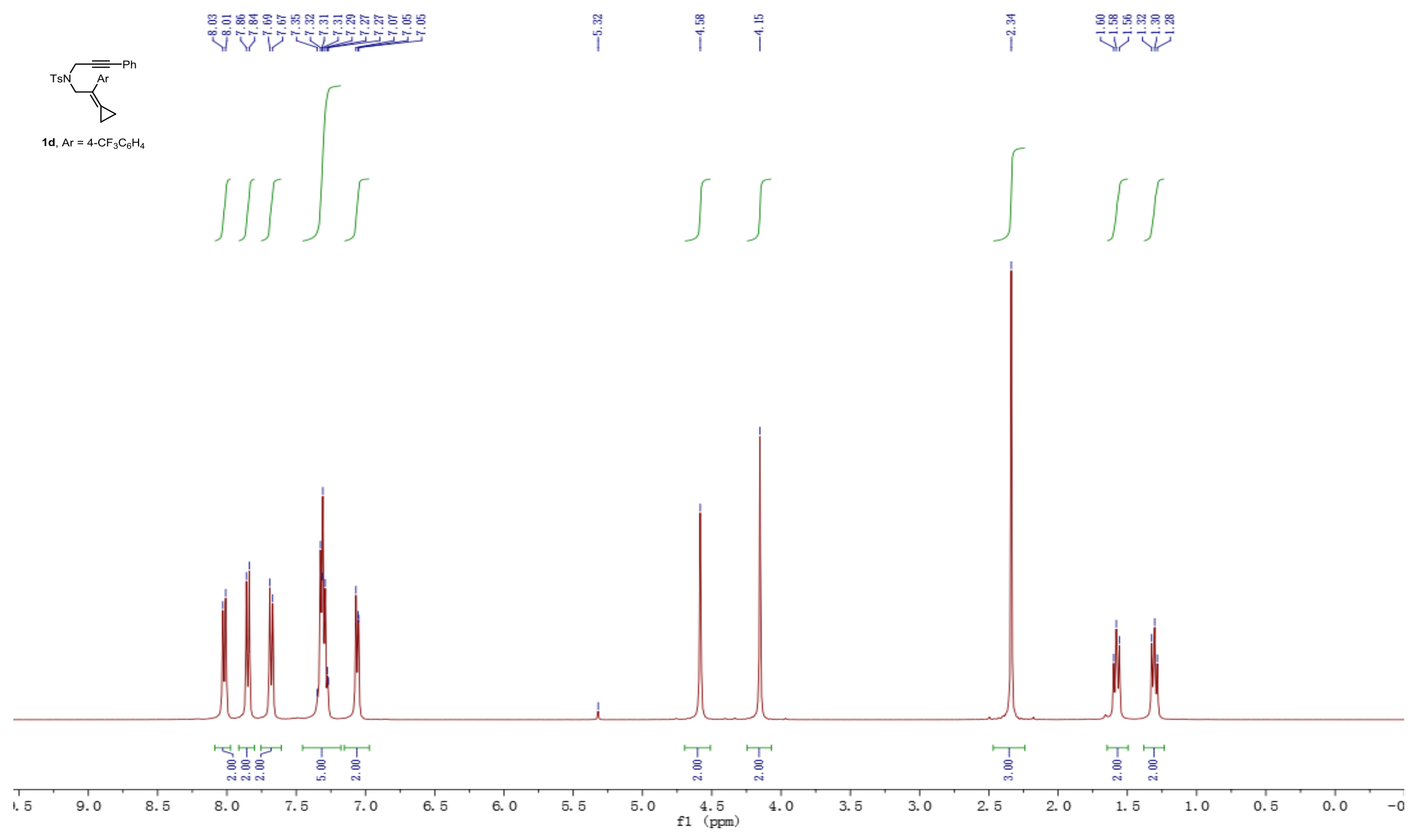




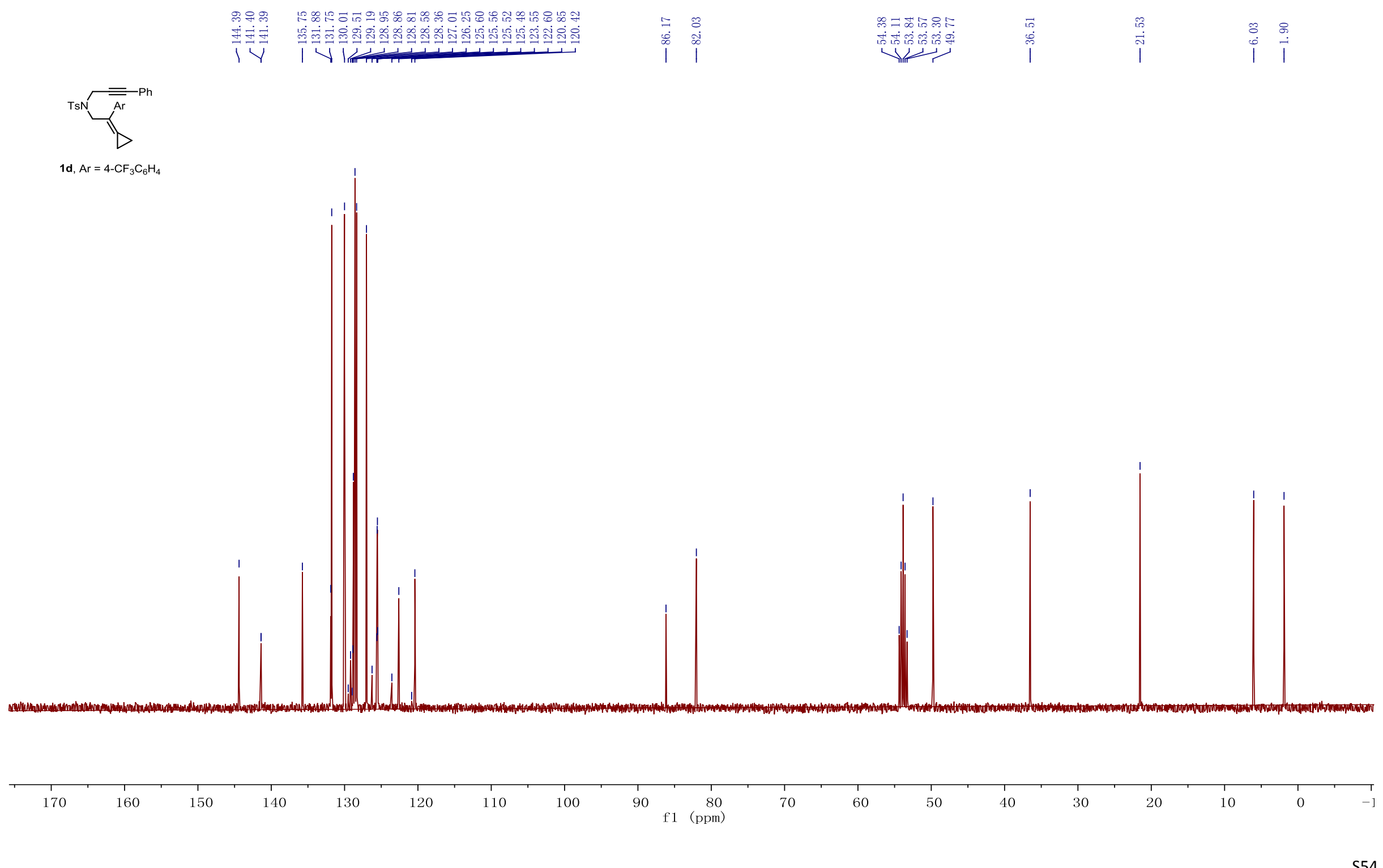




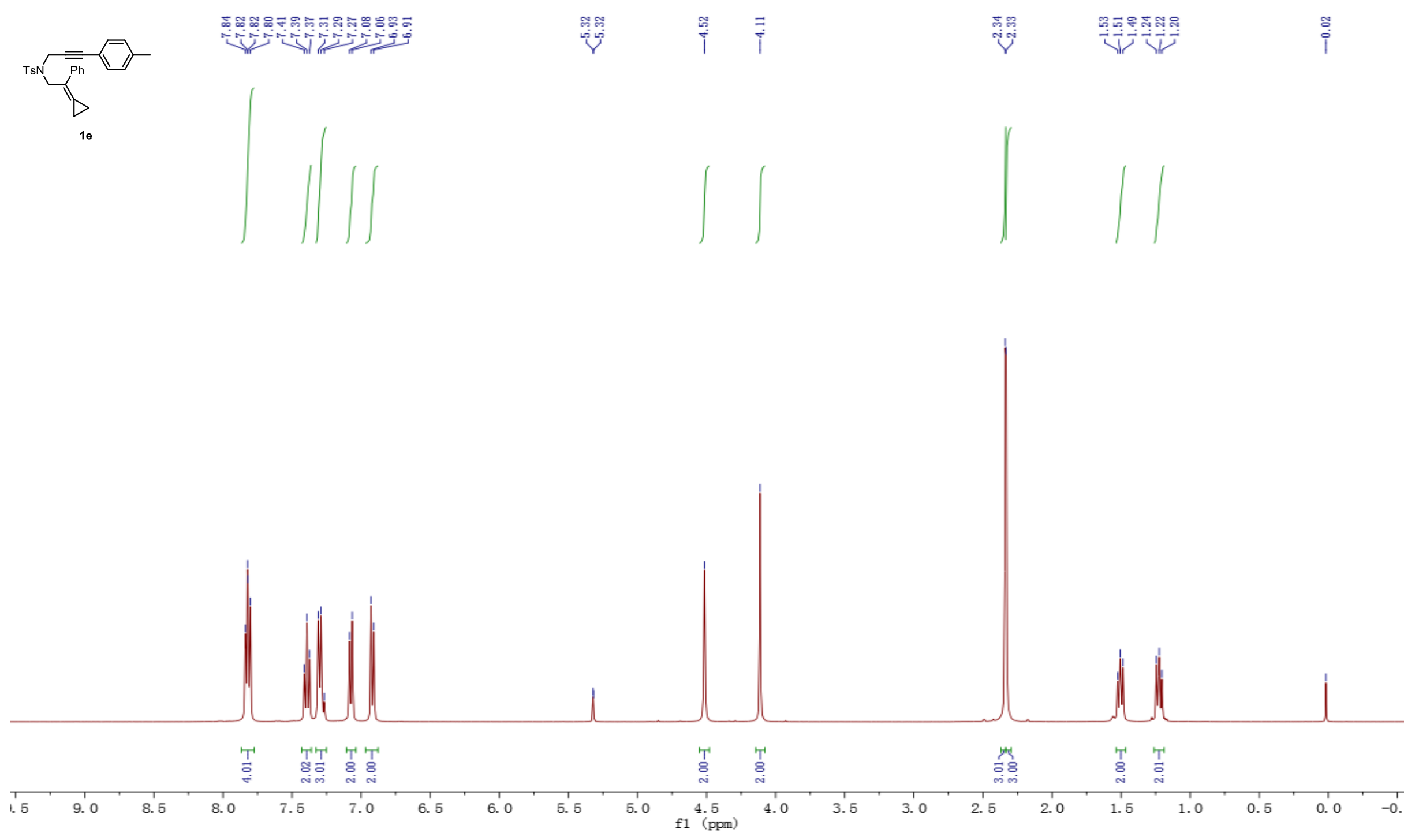



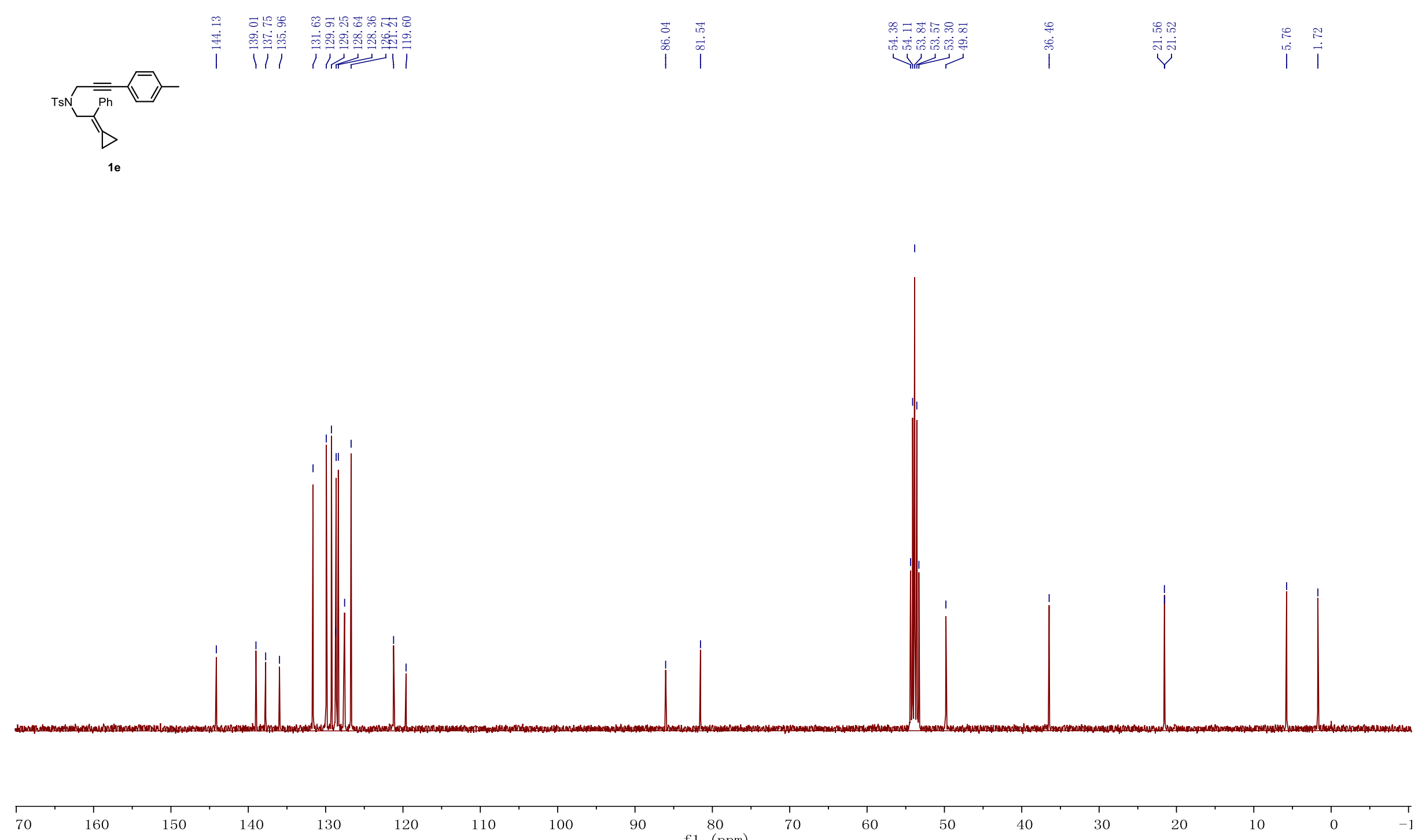

$\mathrm{f} 1 \stackrel{80}{(\mathrm{ppm})}$

70

60

50

40

30 


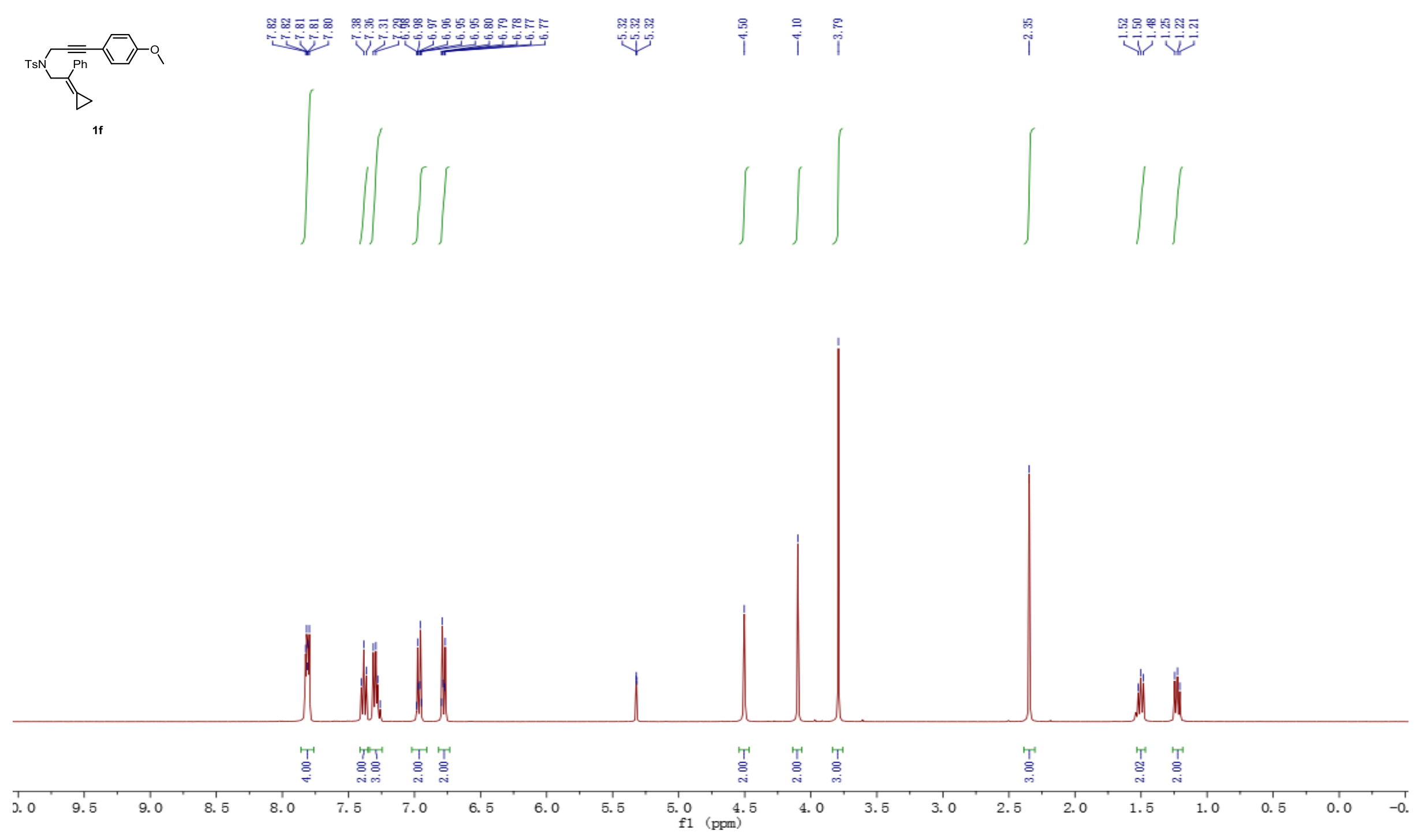




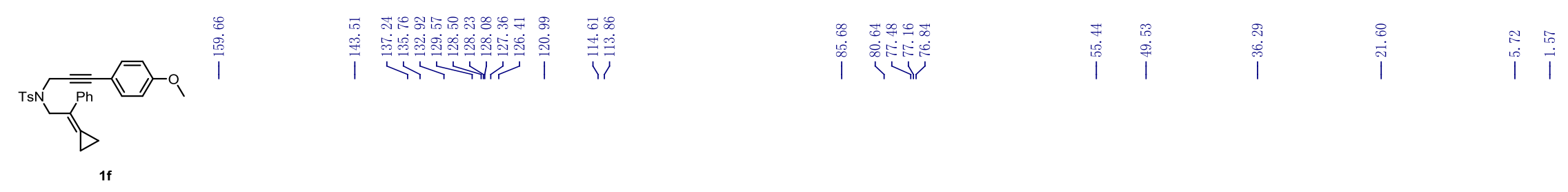
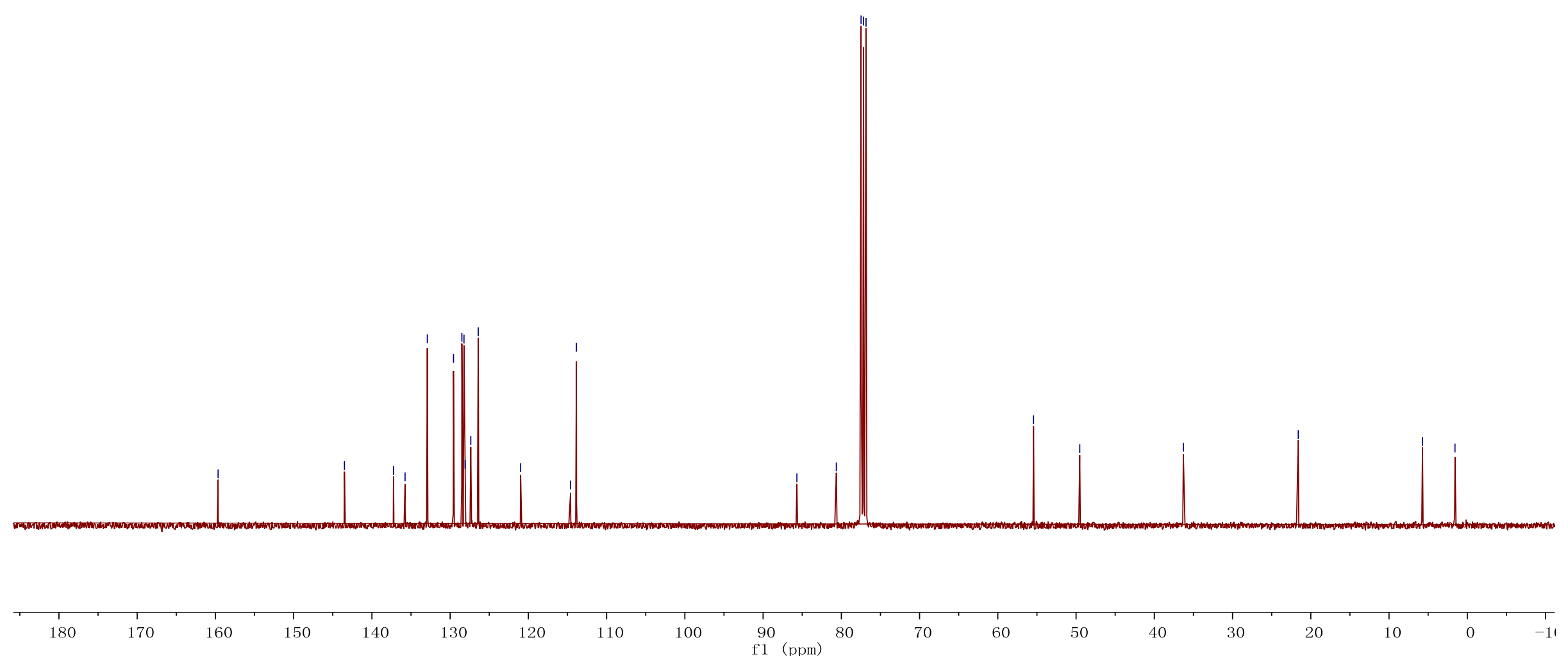

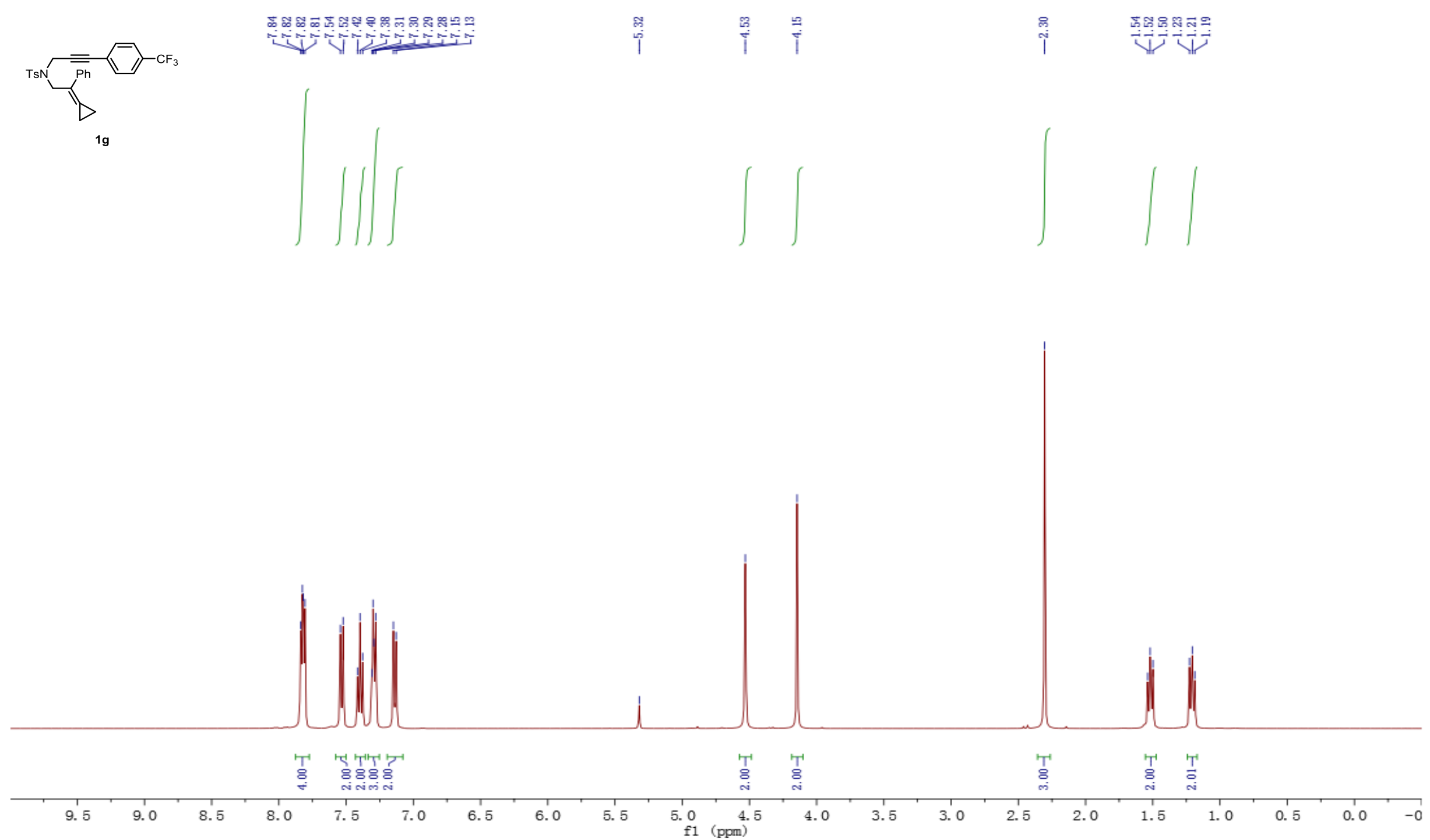

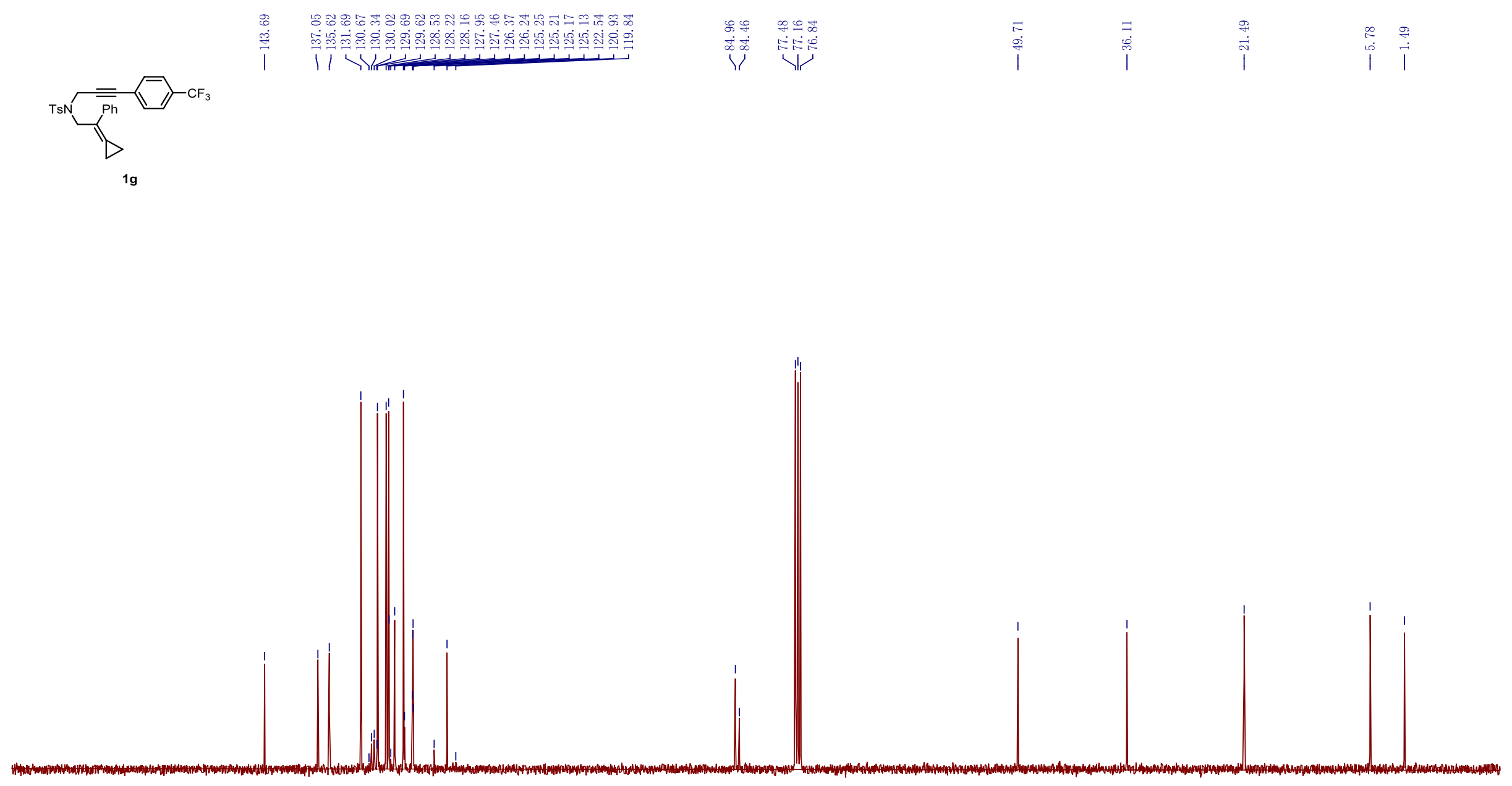

\begin{tabular}{|c|c|c|c|c|c|c|c|c|c|c|c|c|c|c|c|c|c|}
\hline 170 & 160 & 150 & 140 & 130 & 120 & 110 & 100 & 90 & $\begin{array}{c}80 \\
(\mathrm{ppm})\end{array}$ & 70 & 60 & 50 & 40 & 30 & 20 & 10 & 0 \\
\hline
\end{tabular}




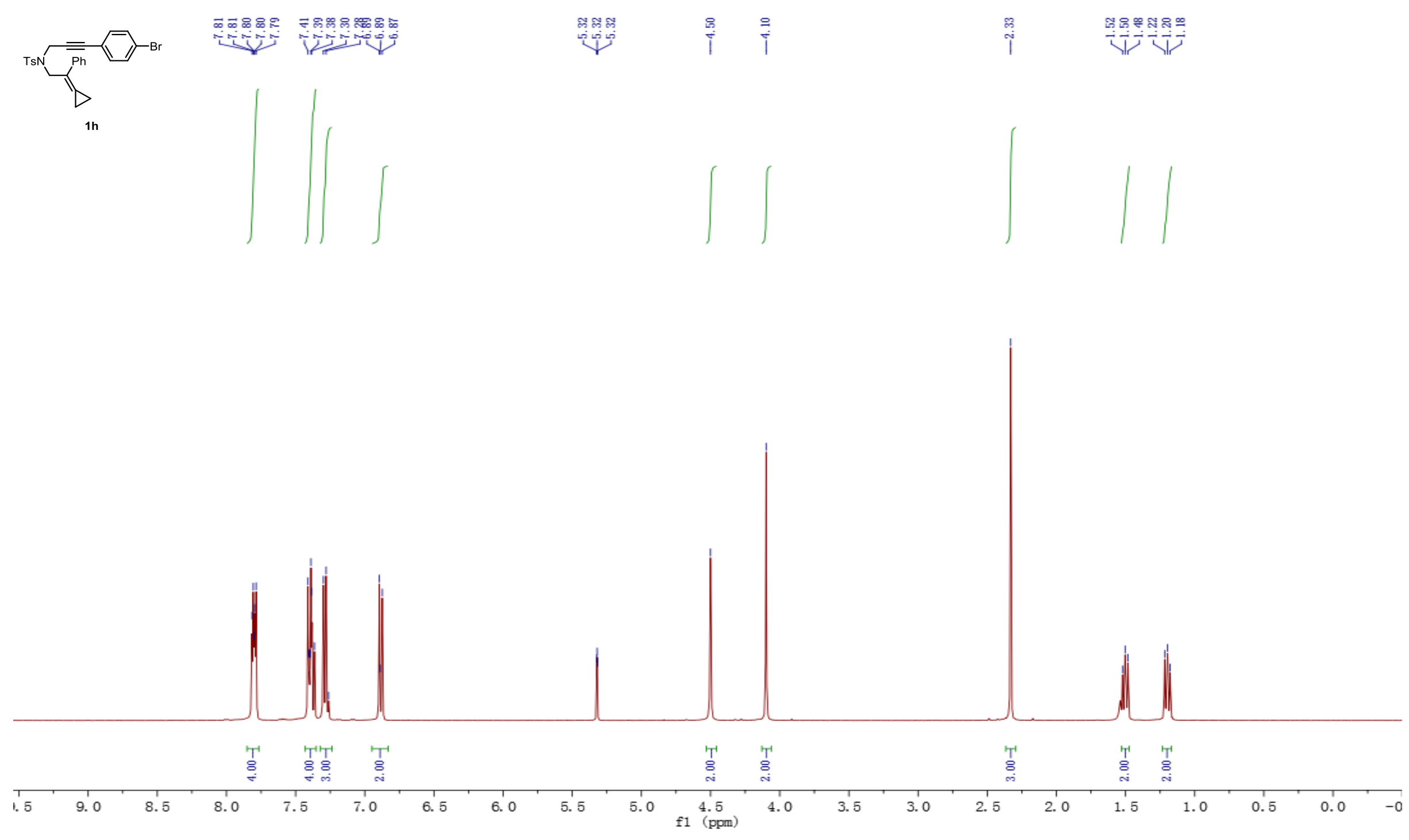



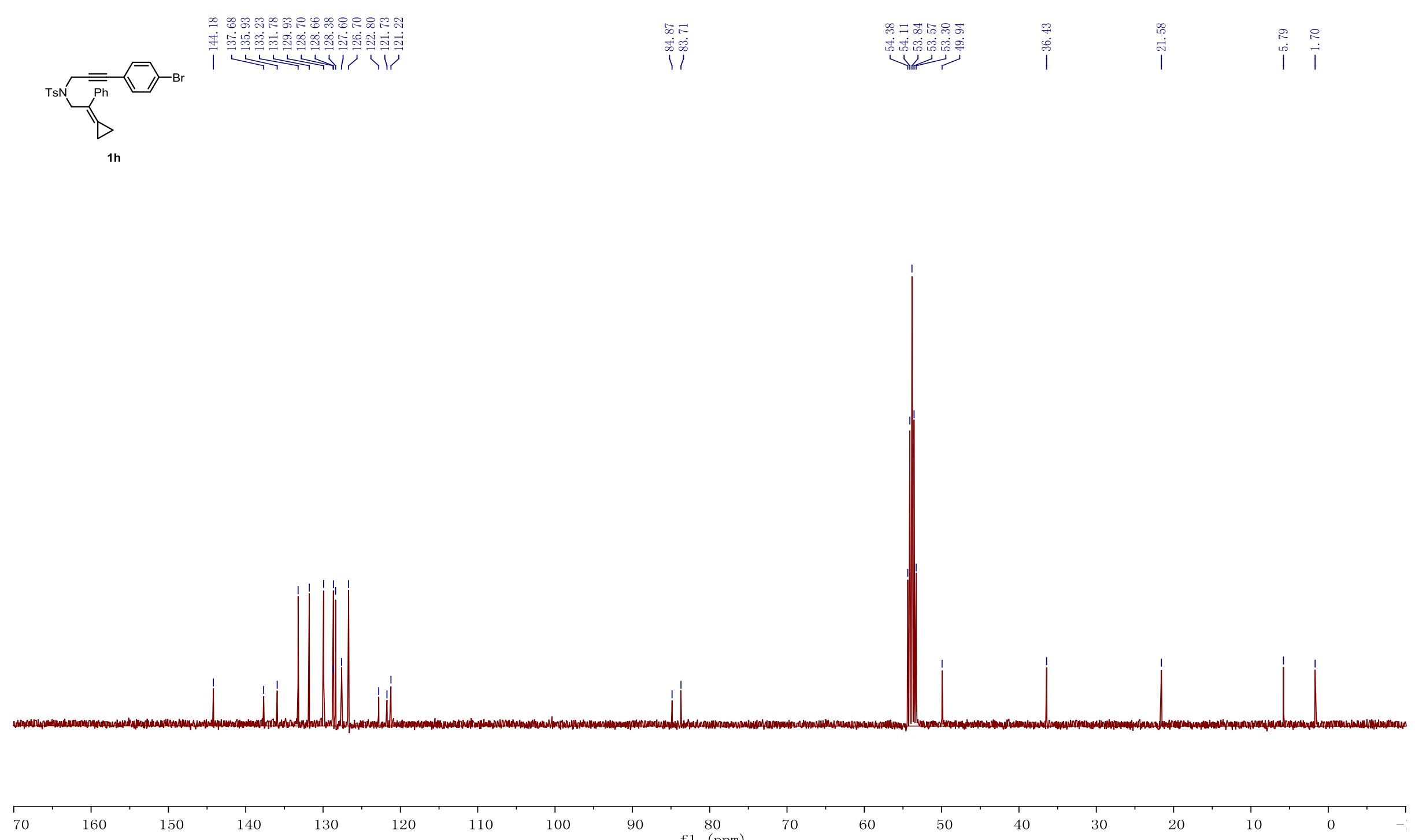

90 $\mathrm{f} 1 \stackrel{80}{(\mathrm{ppm})}$ 70

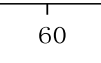

50

40 30 


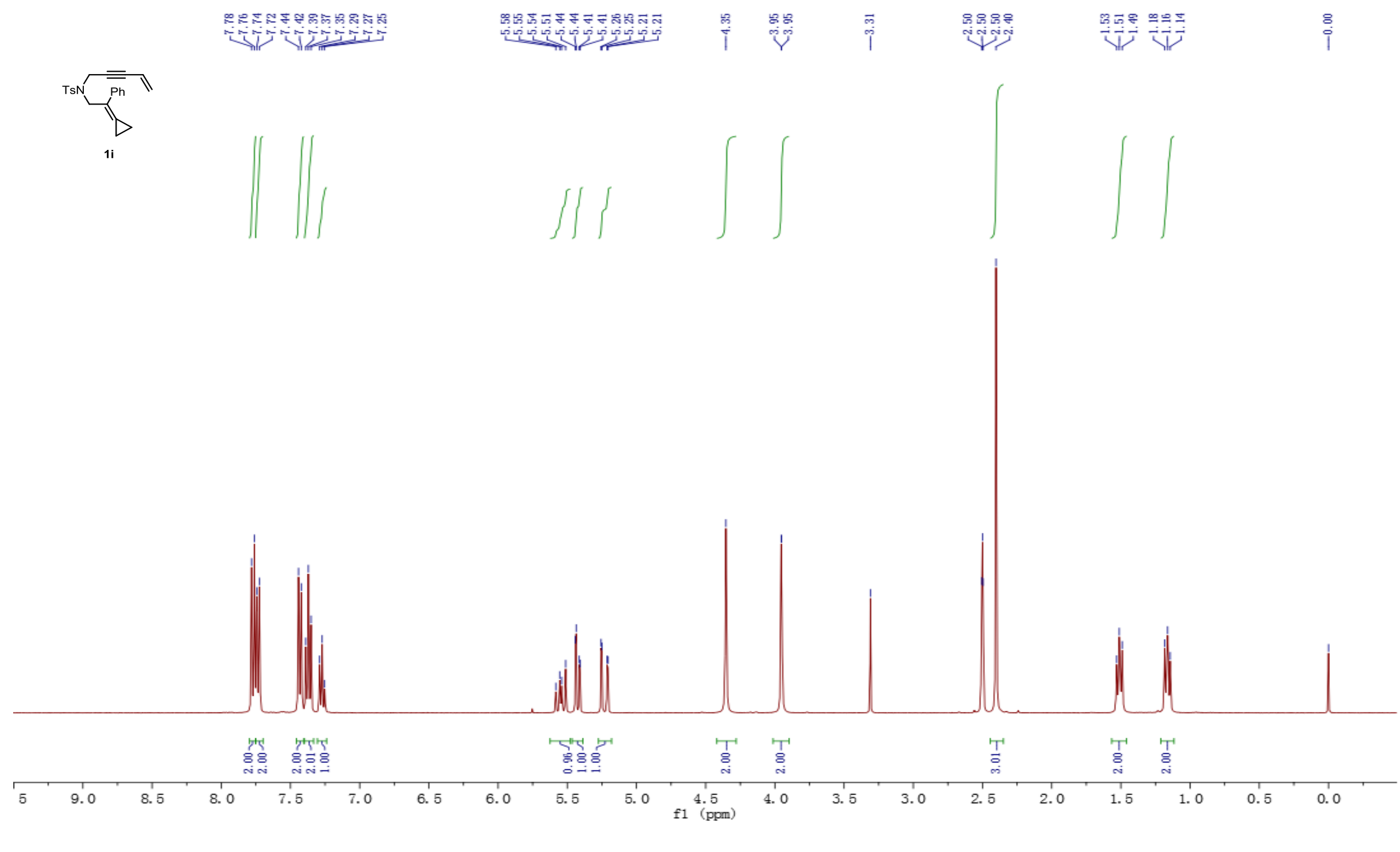




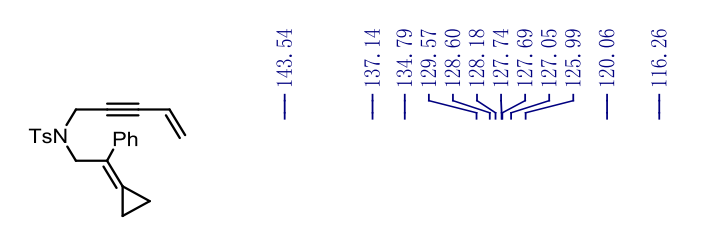

$1 \mathrm{i}$

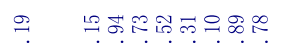

क

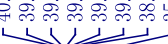

iิ

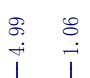
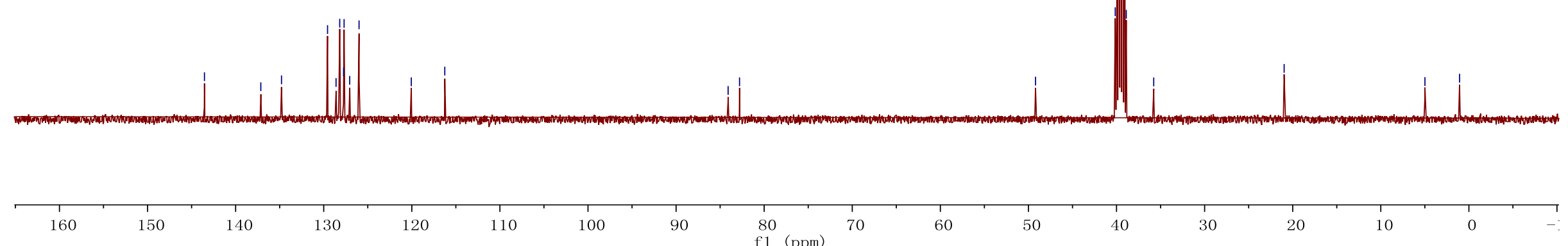

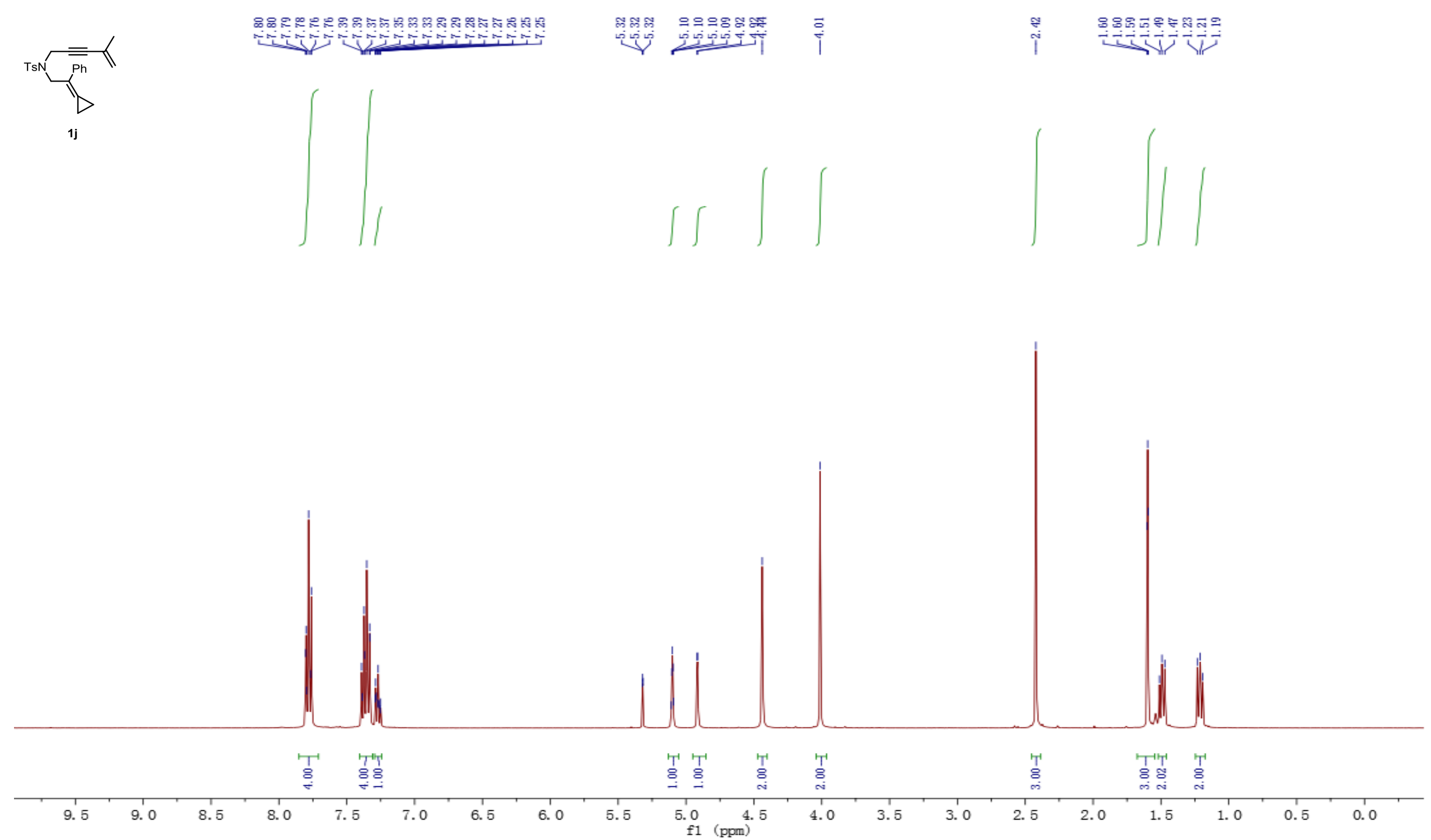

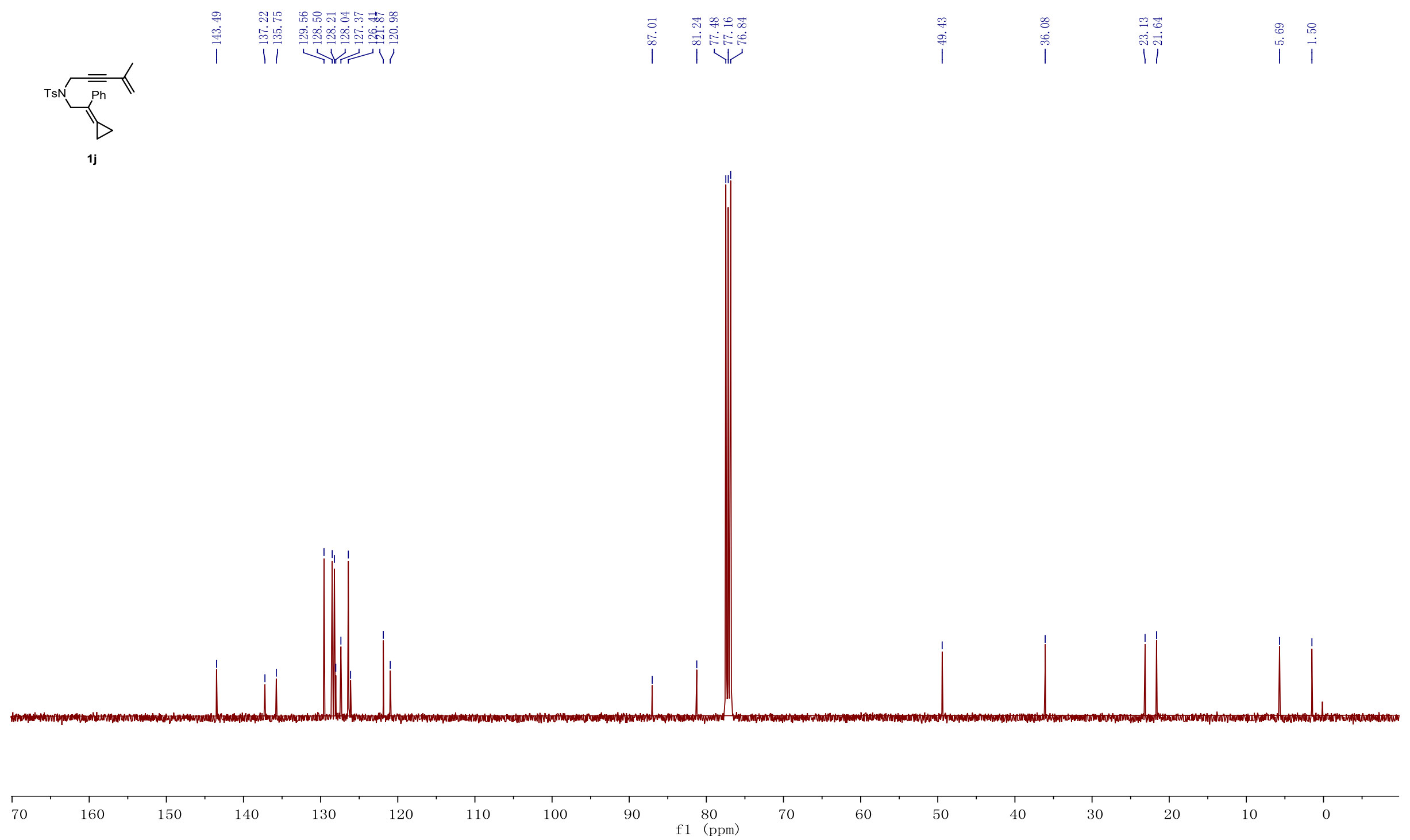


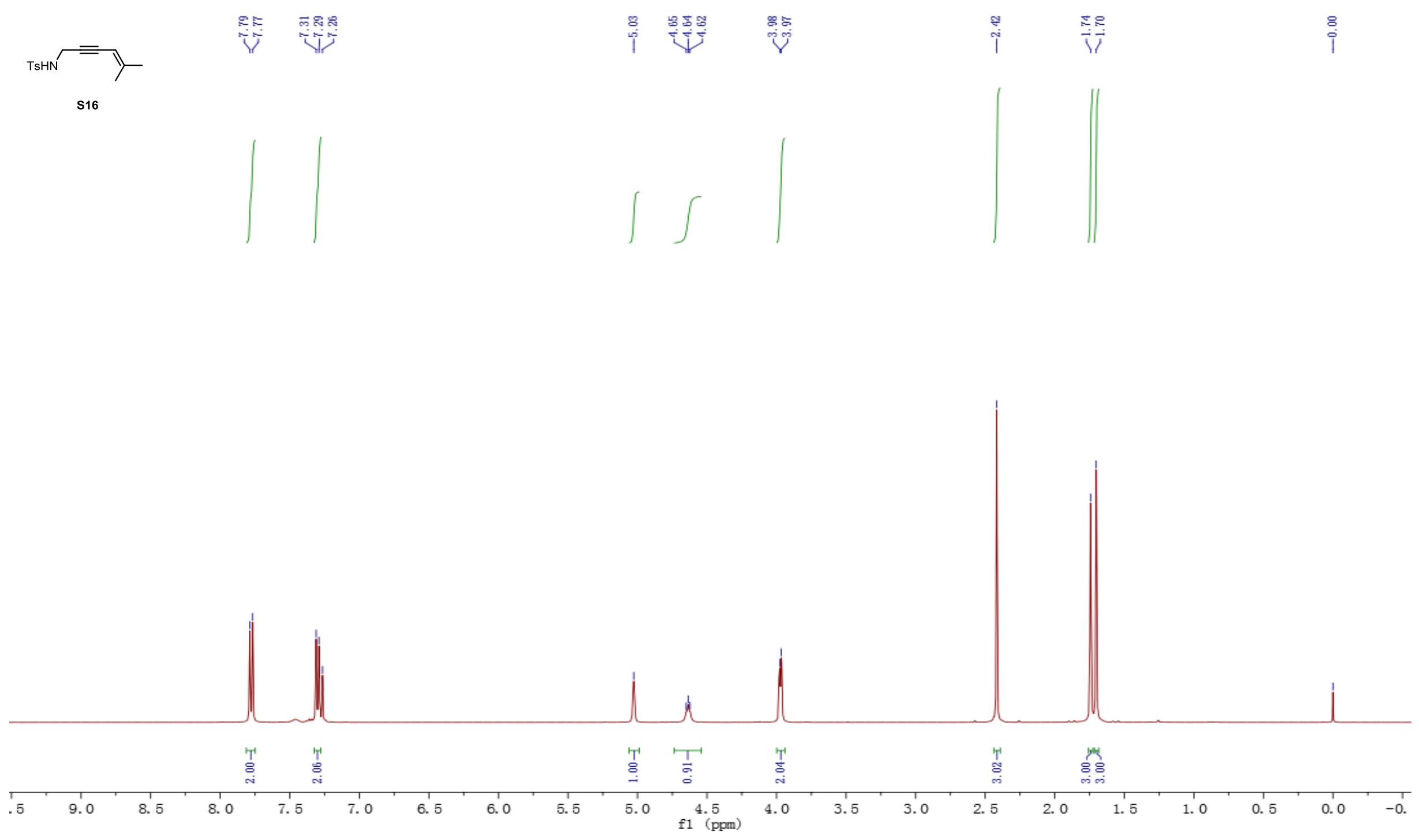



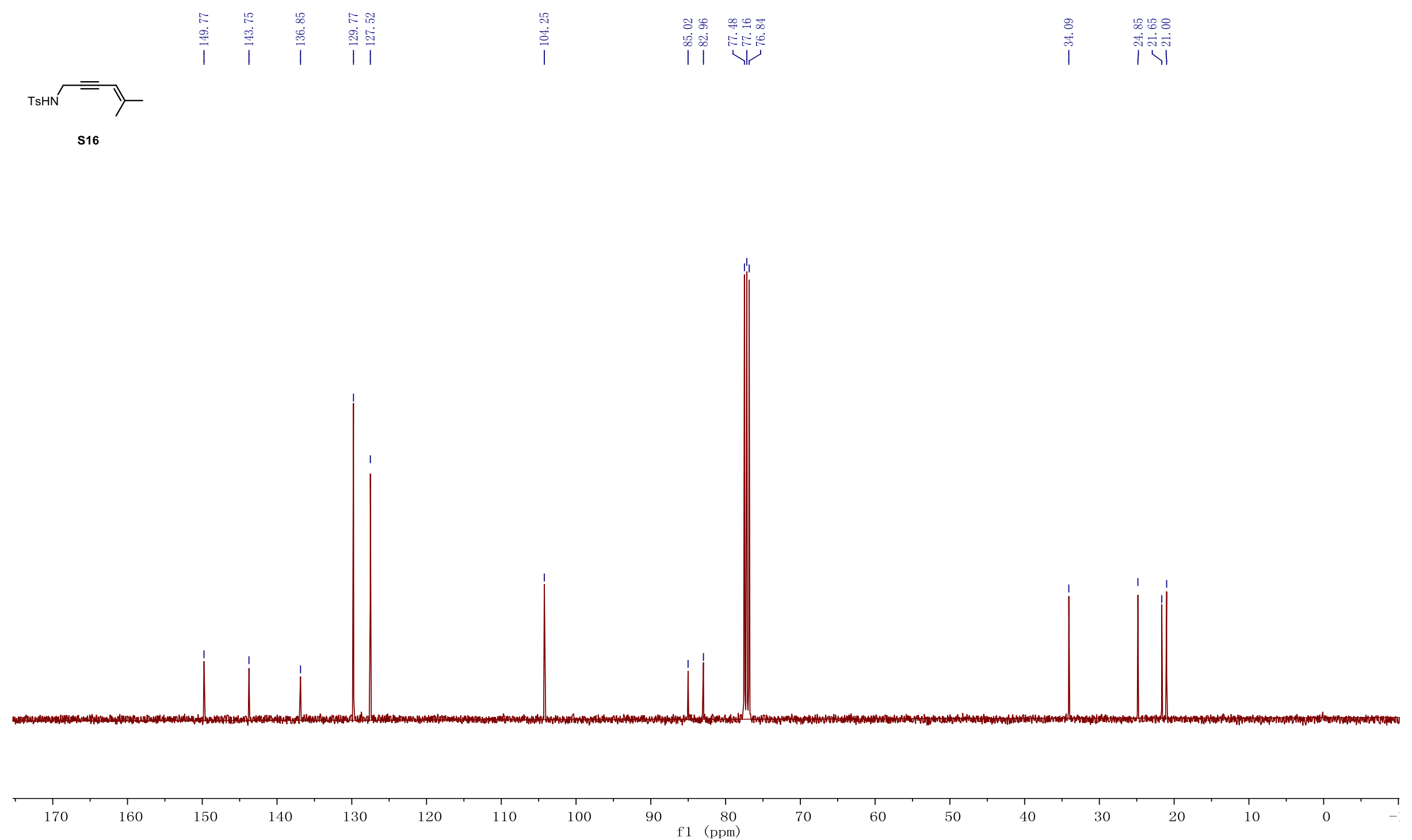


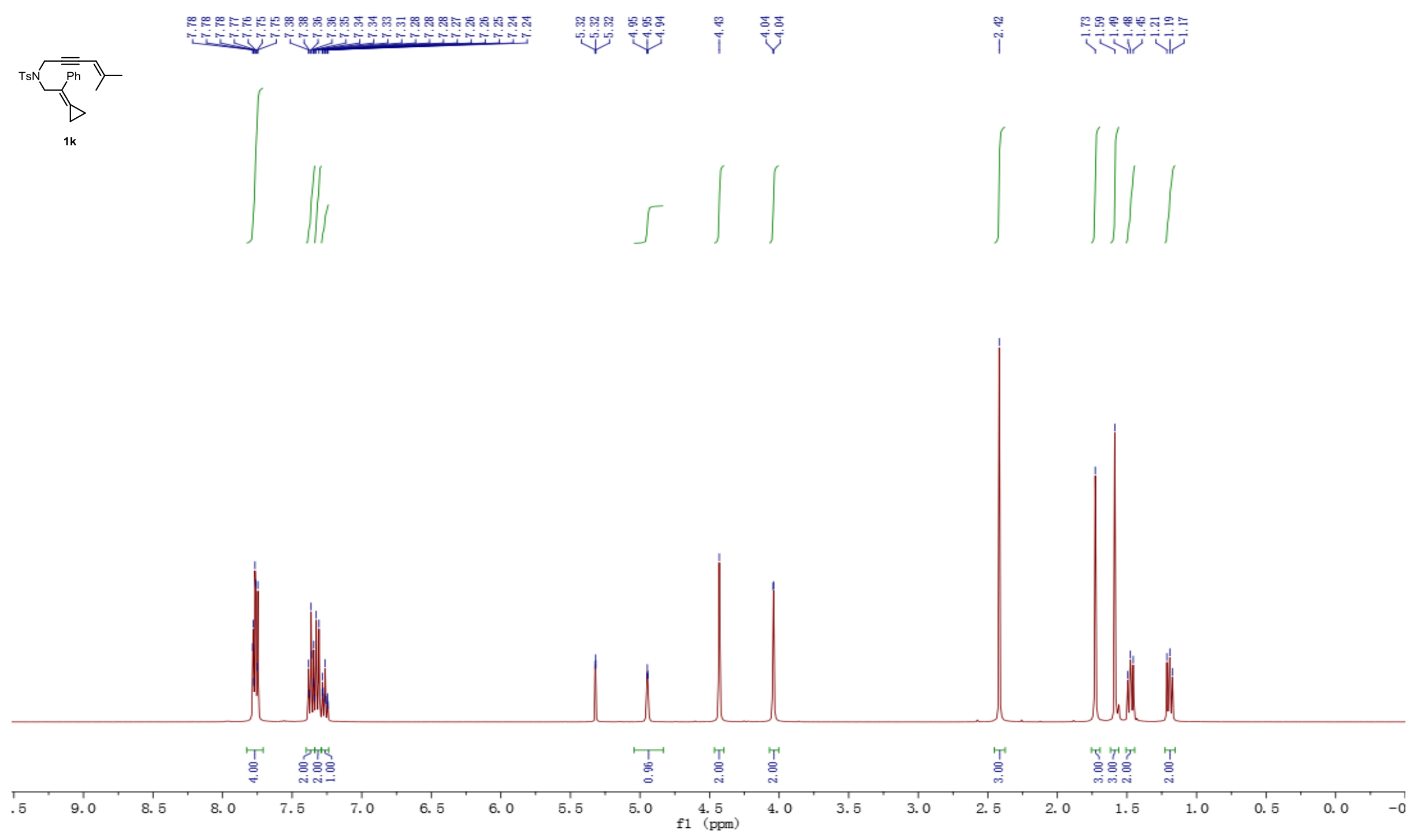



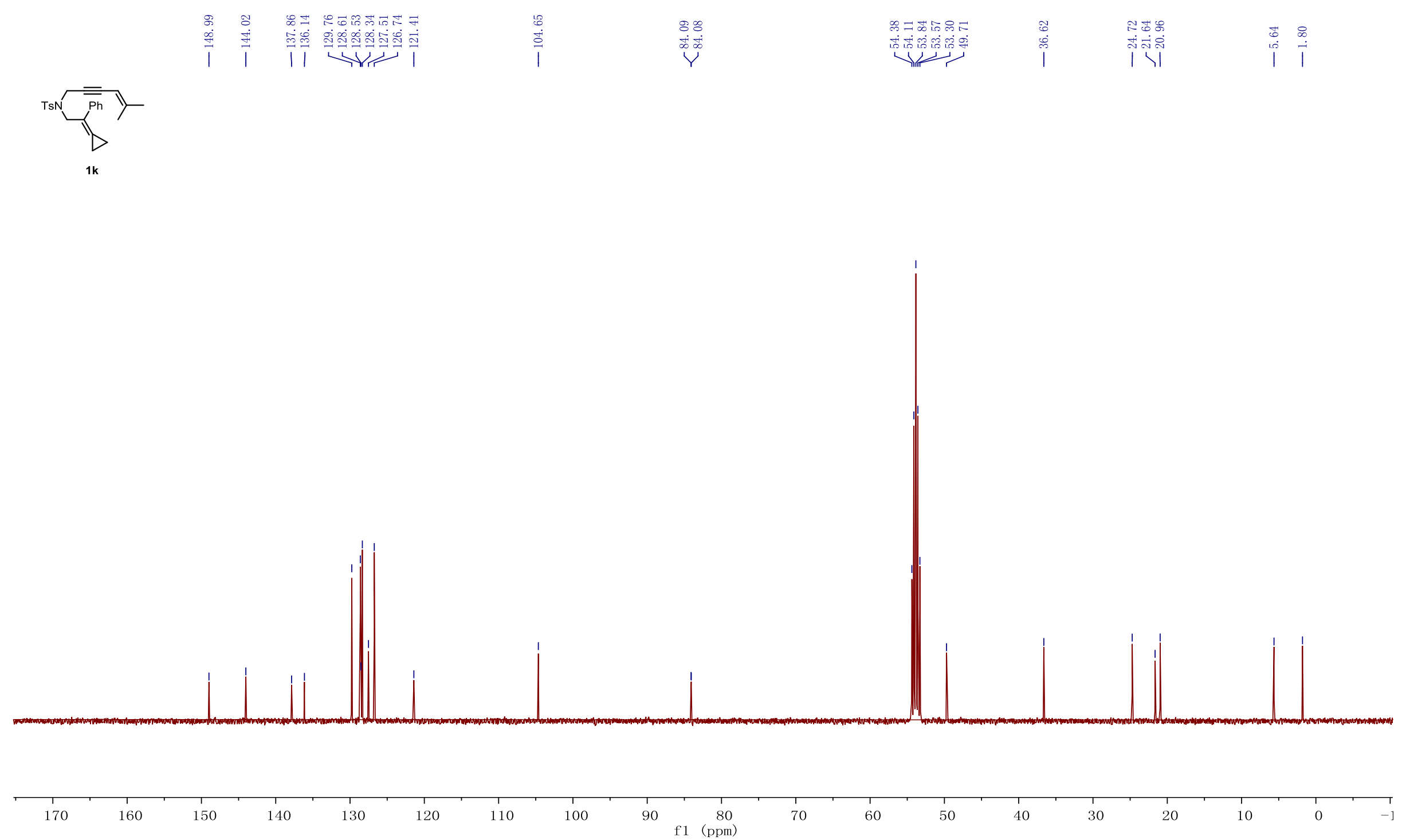

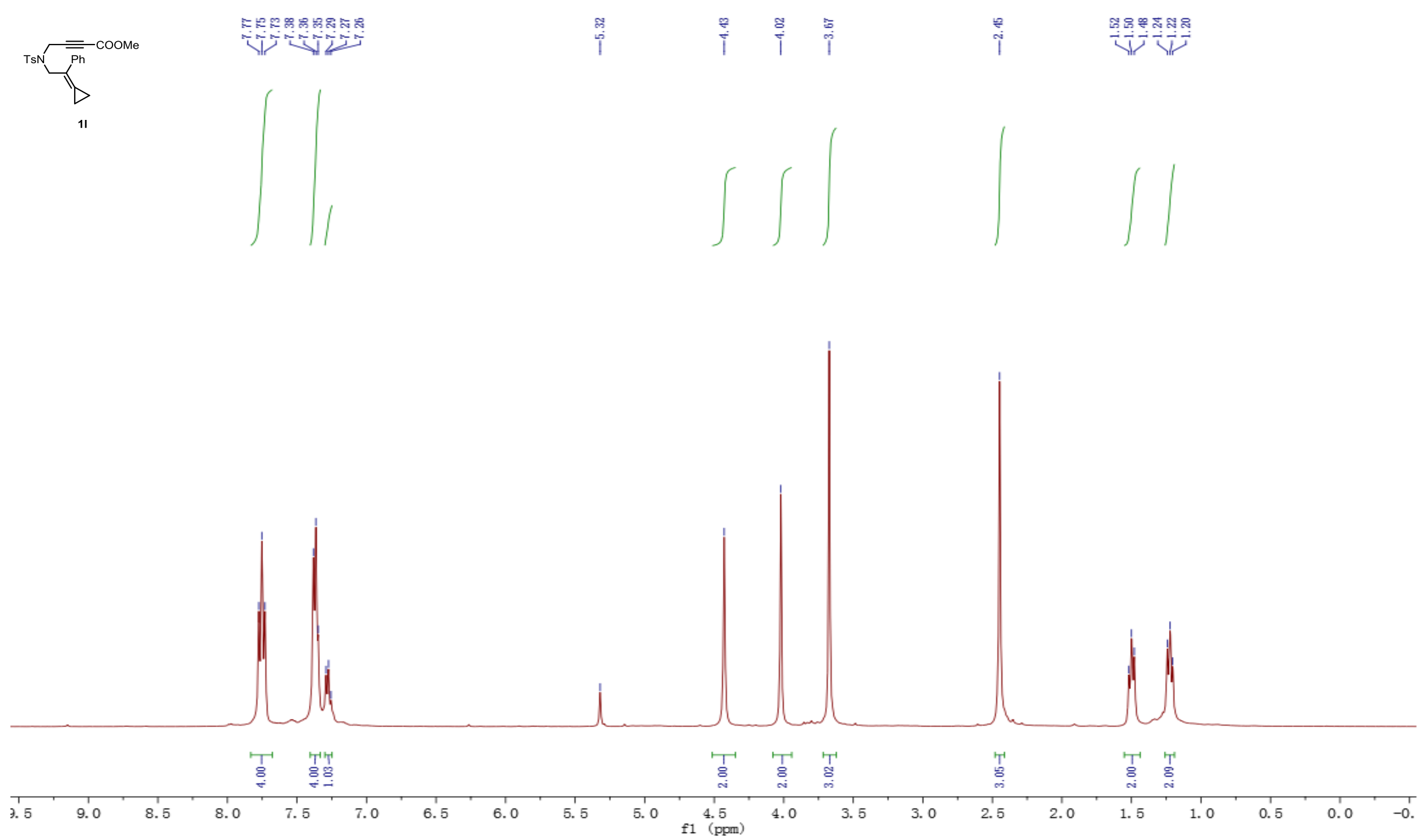


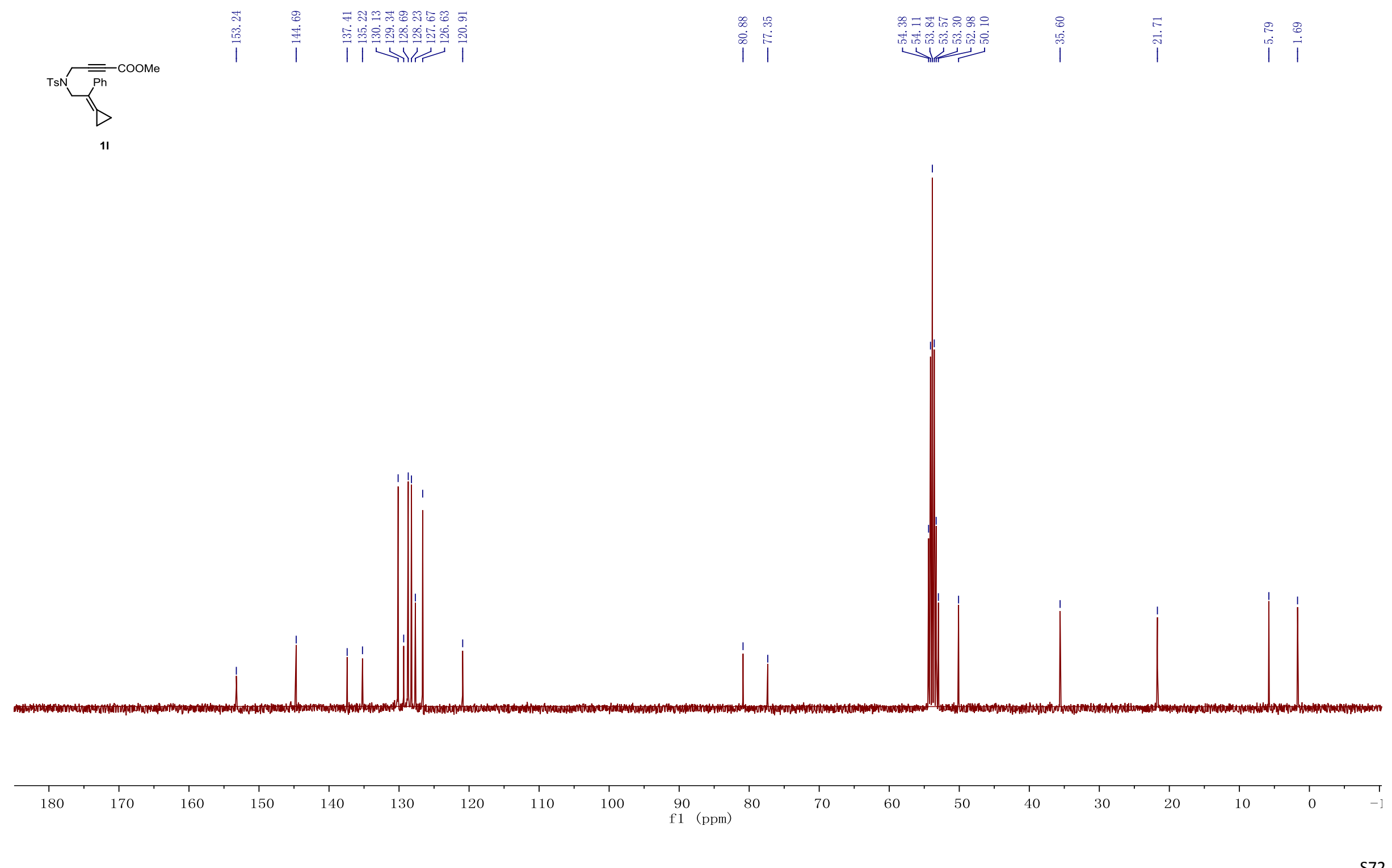




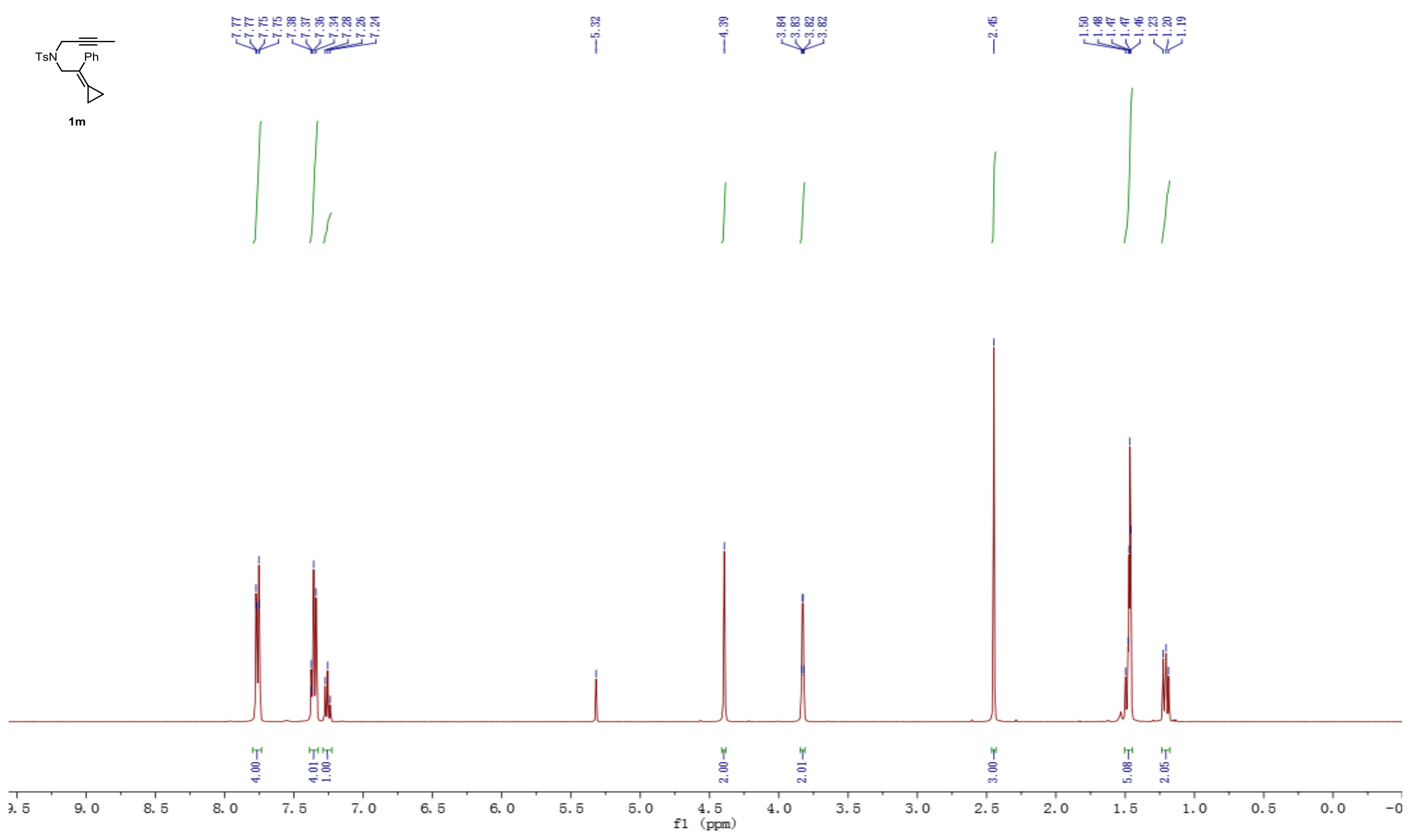



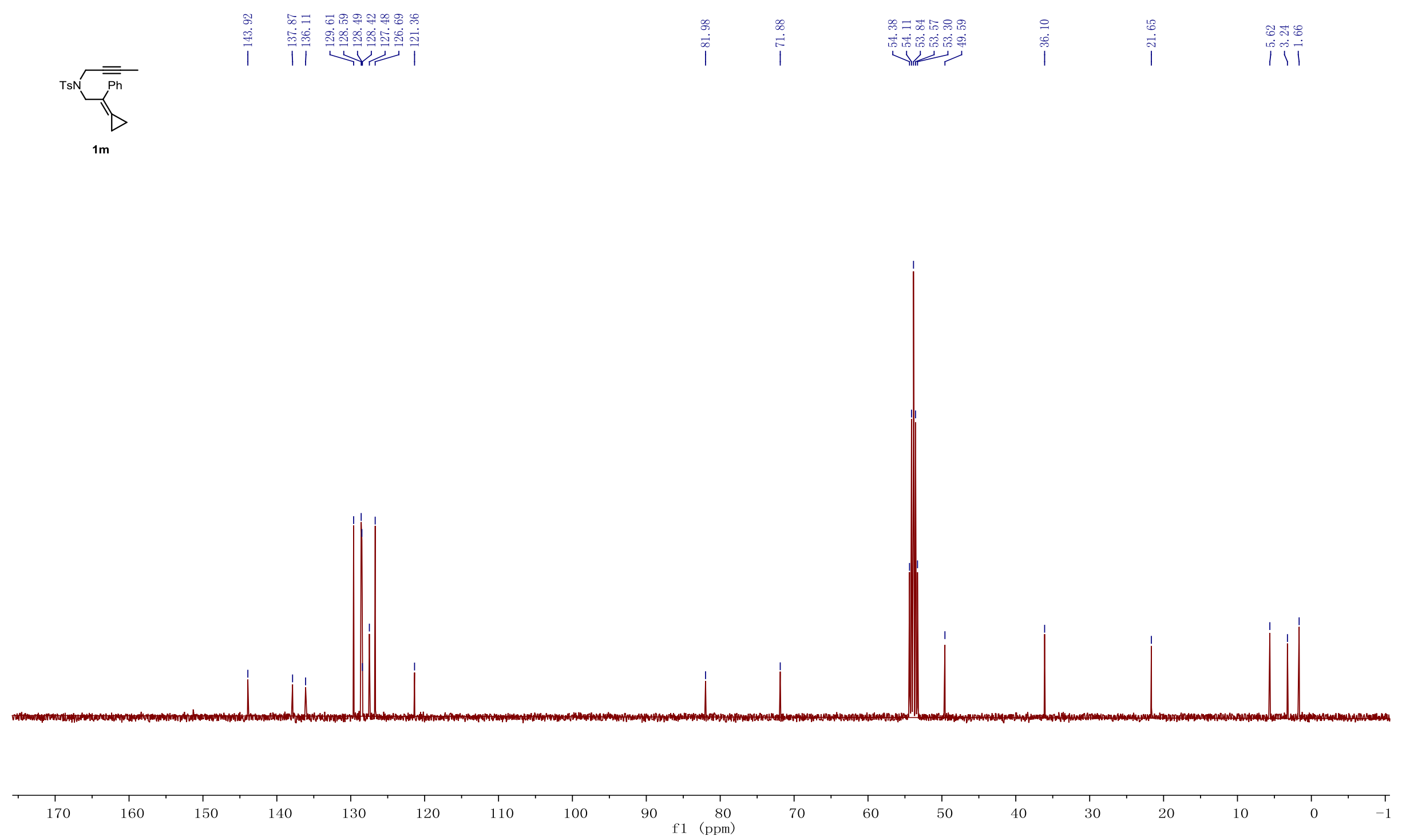


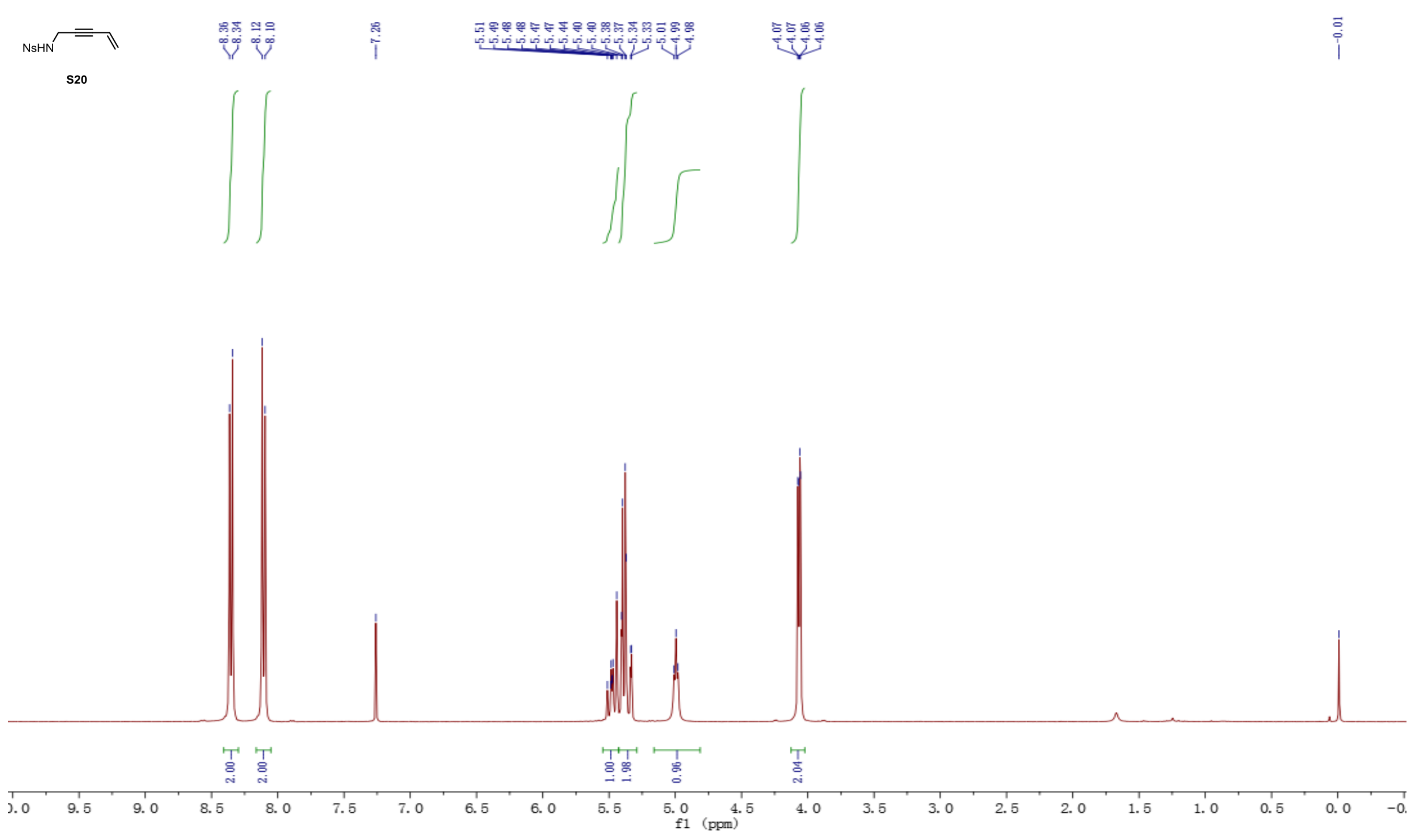




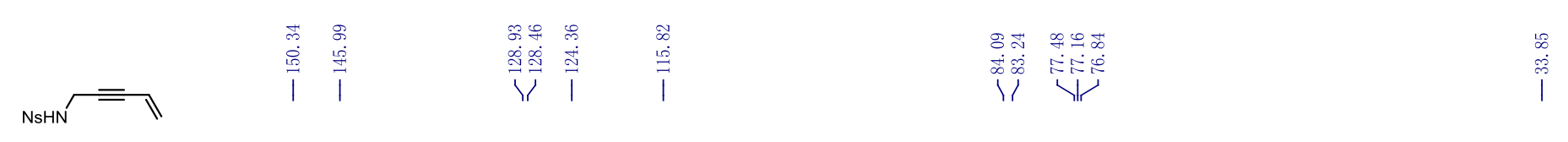

S20
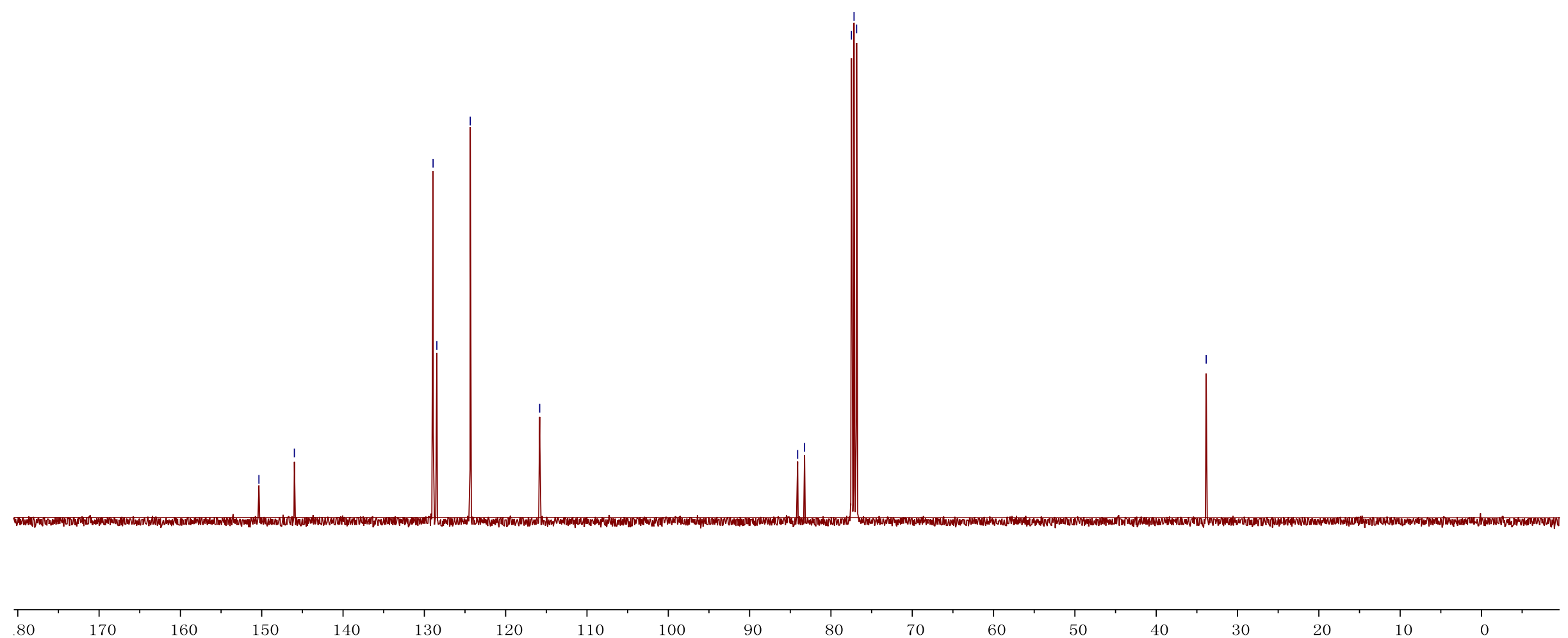

$50 \quad 140$
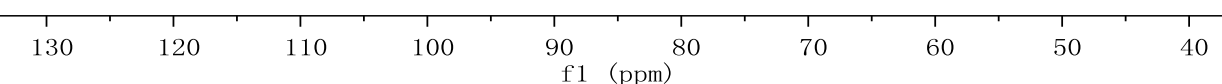

30 

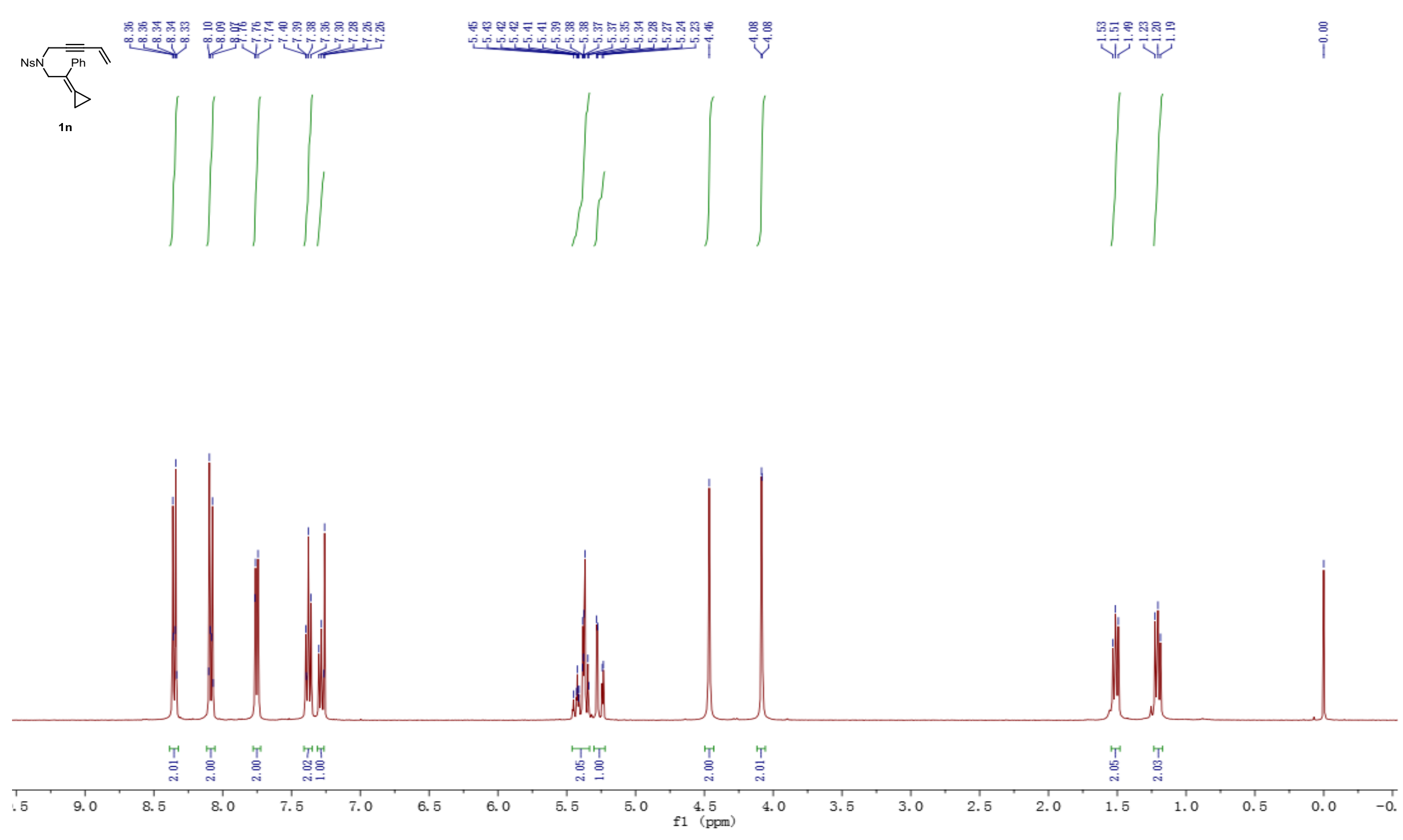

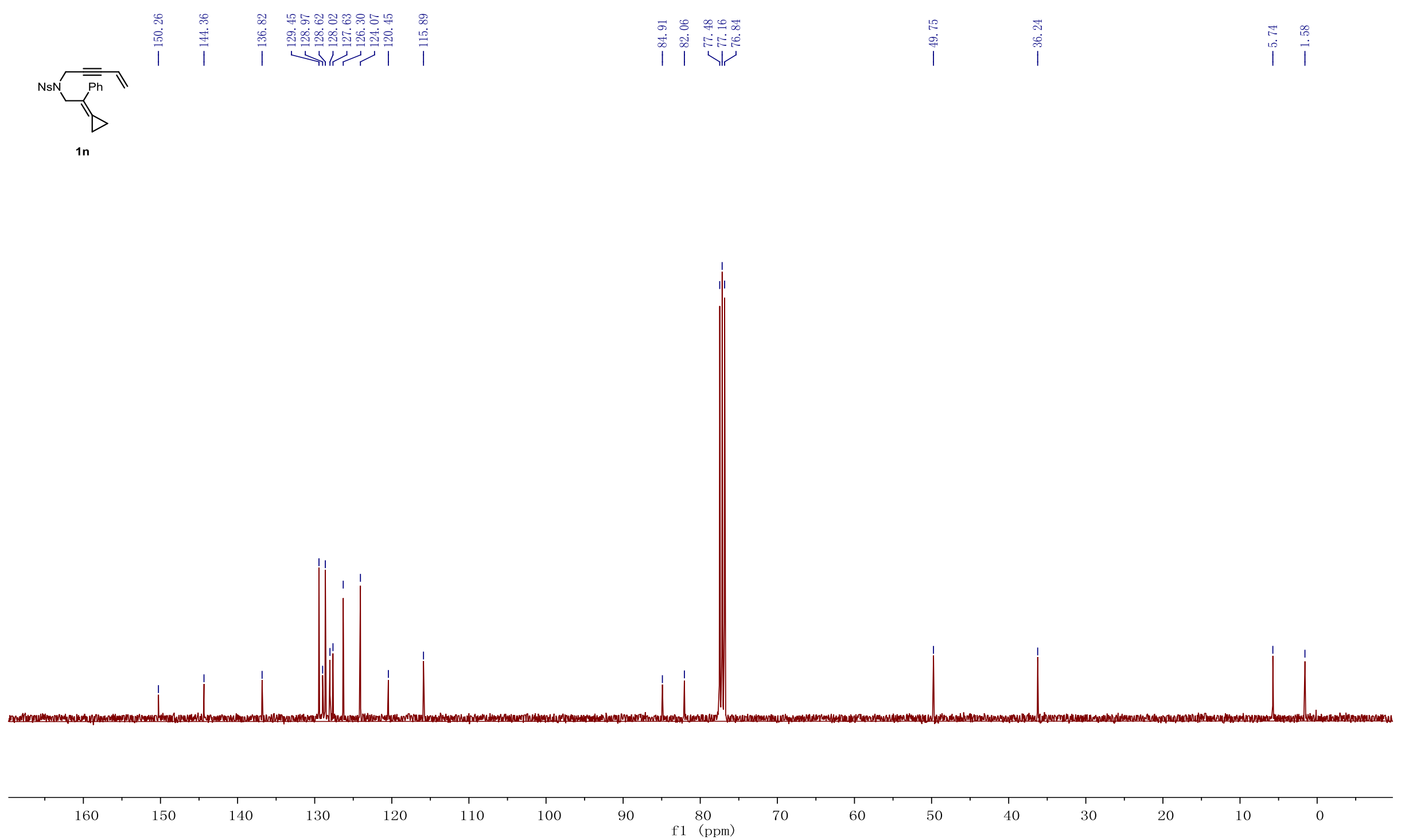


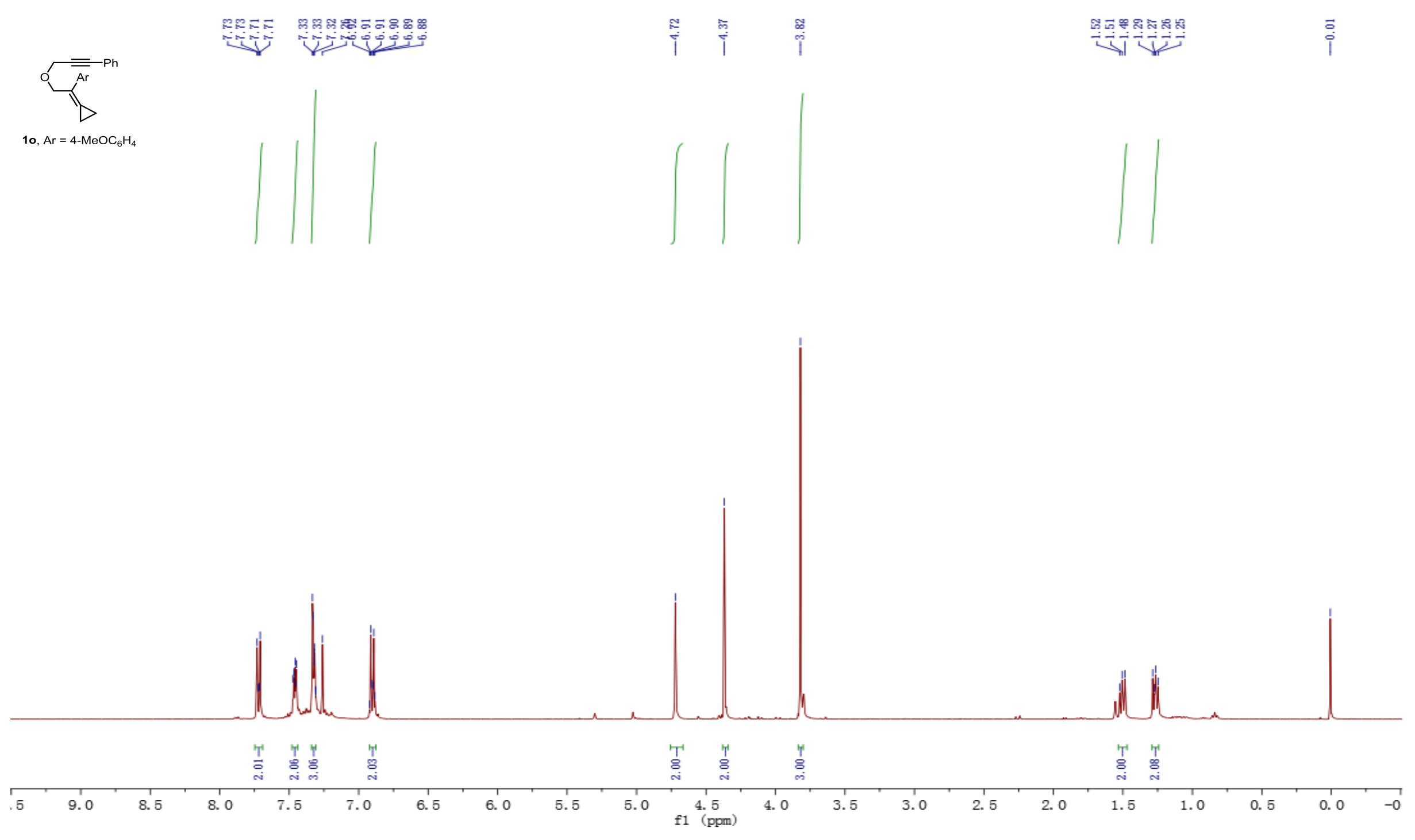




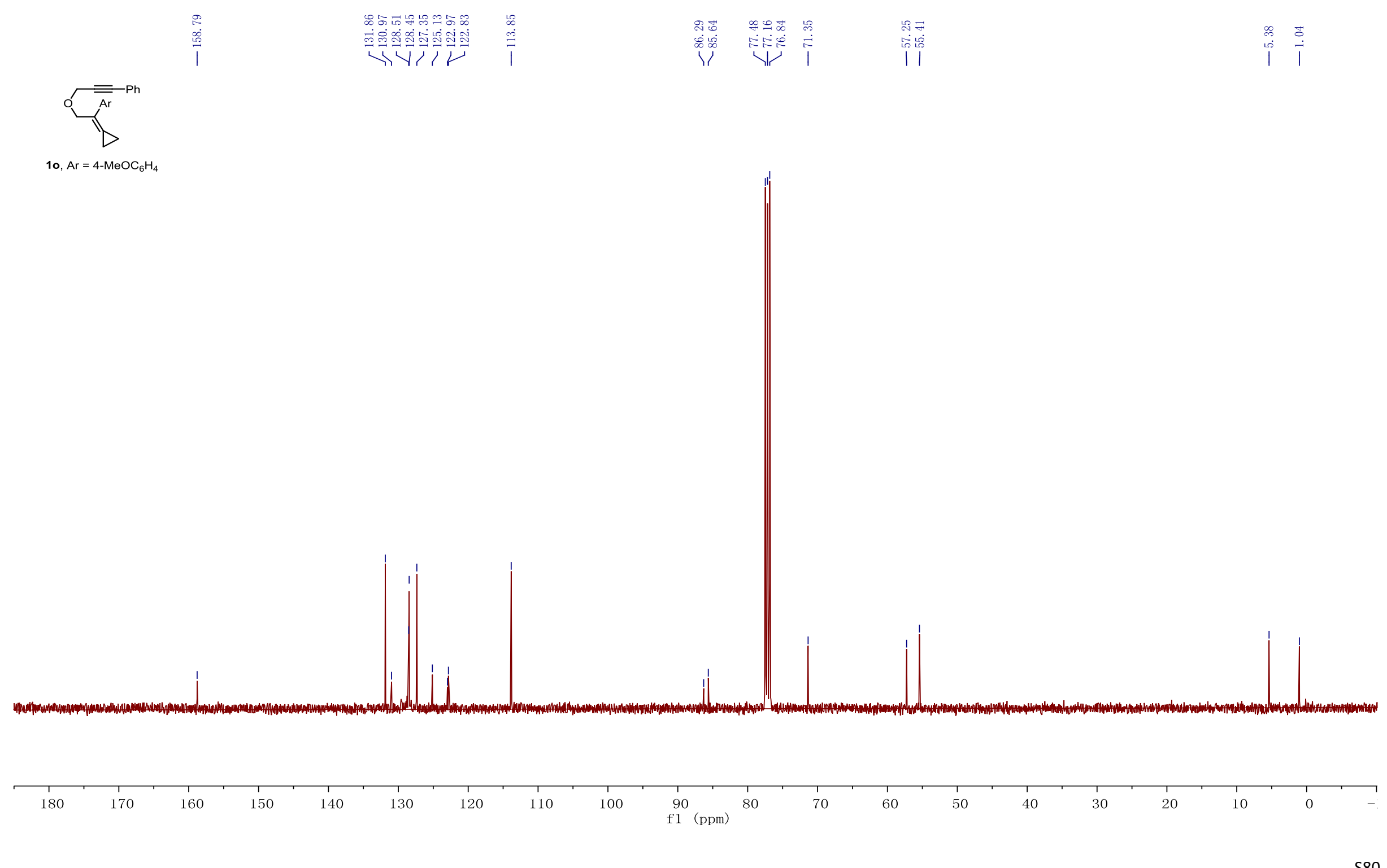




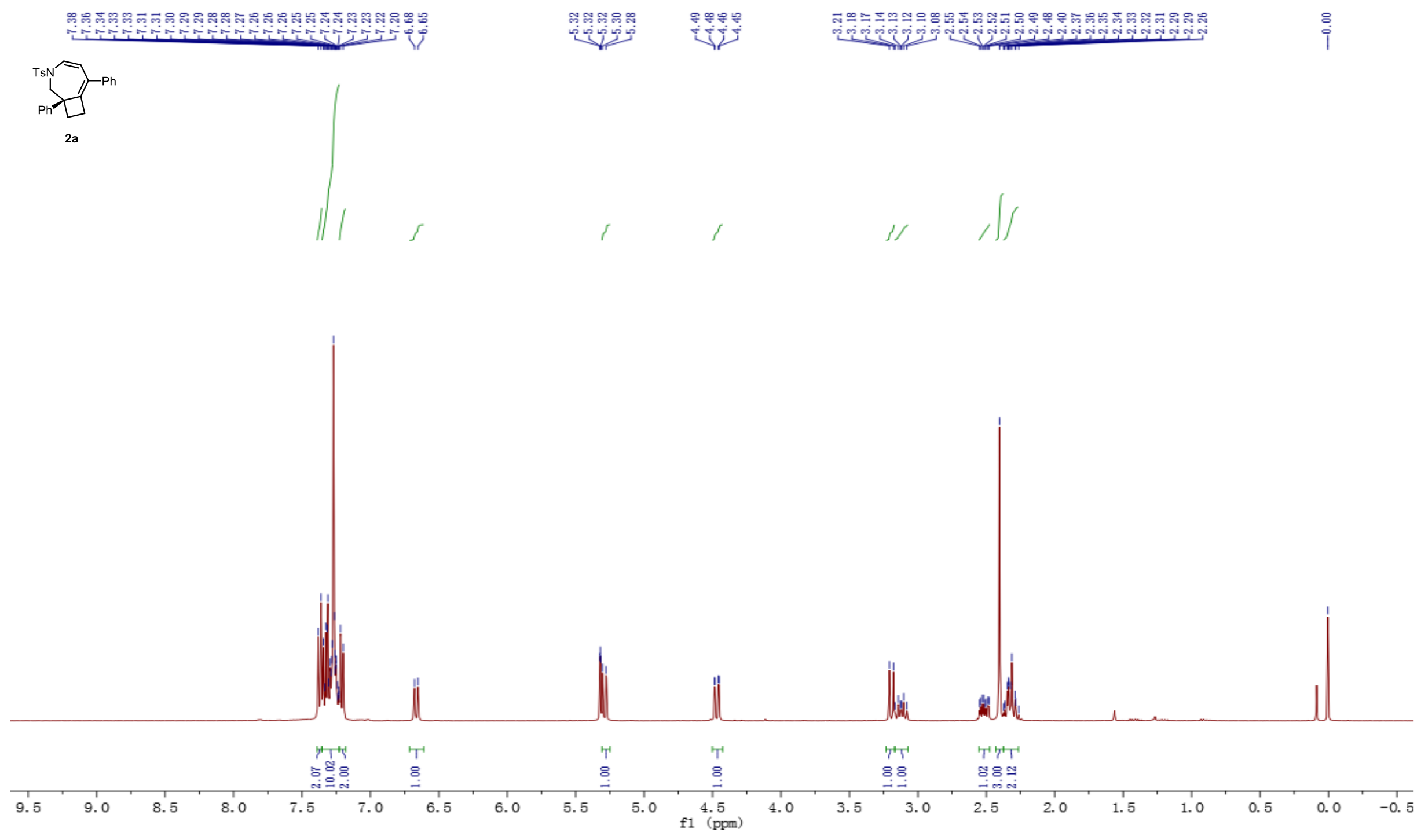




\begin{tabular}{|c|c|c|c|c|c|}
\hline 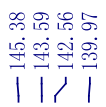 & 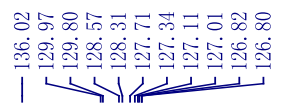 & $\begin{array}{l}\text { in } \\
\text { : } \\
0 \\
0\end{array}$ & 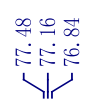 & 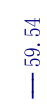 & \\
\hline
\end{tabular}

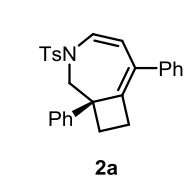

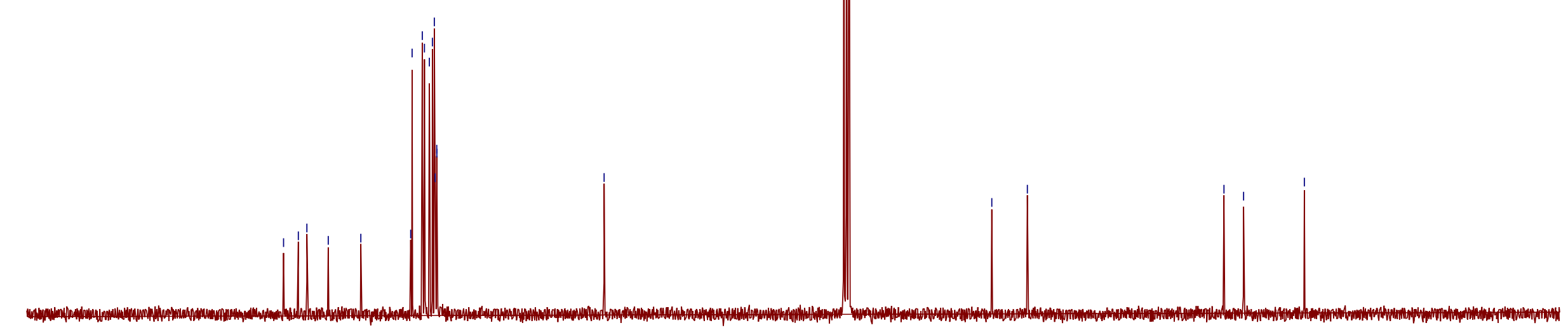

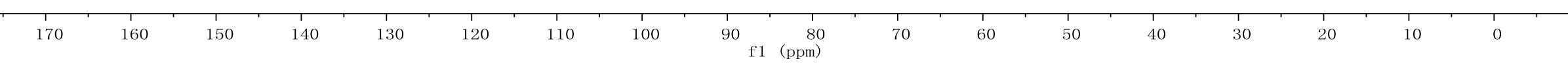




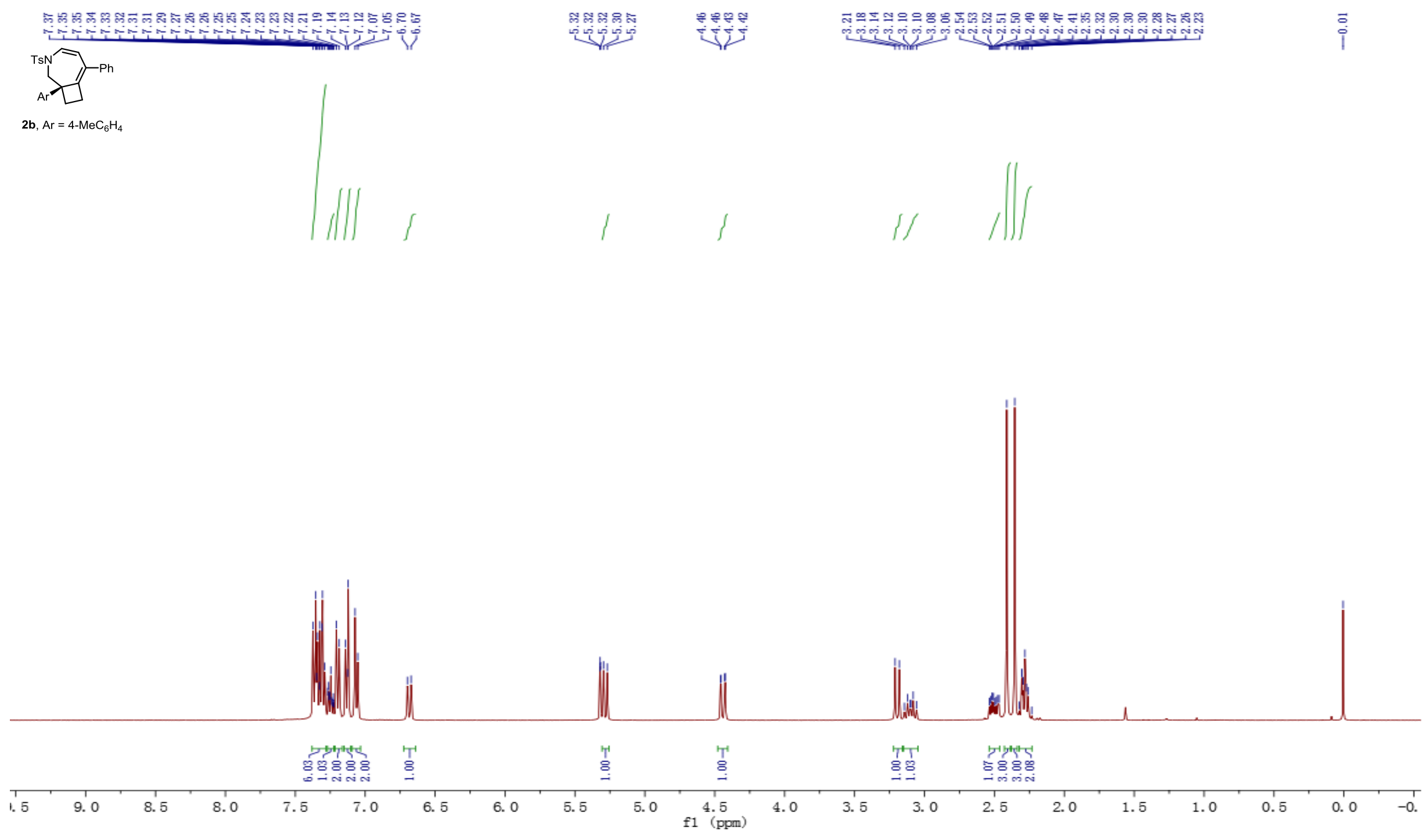




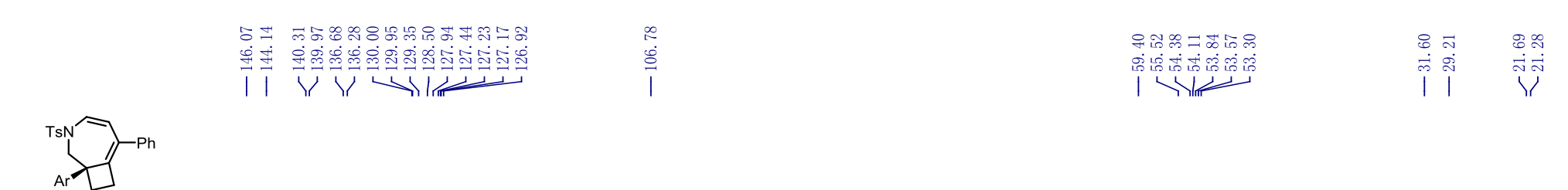

2b, $\mathrm{Ar}=4-\mathrm{MeC}_{6} \mathrm{H}_{4}$

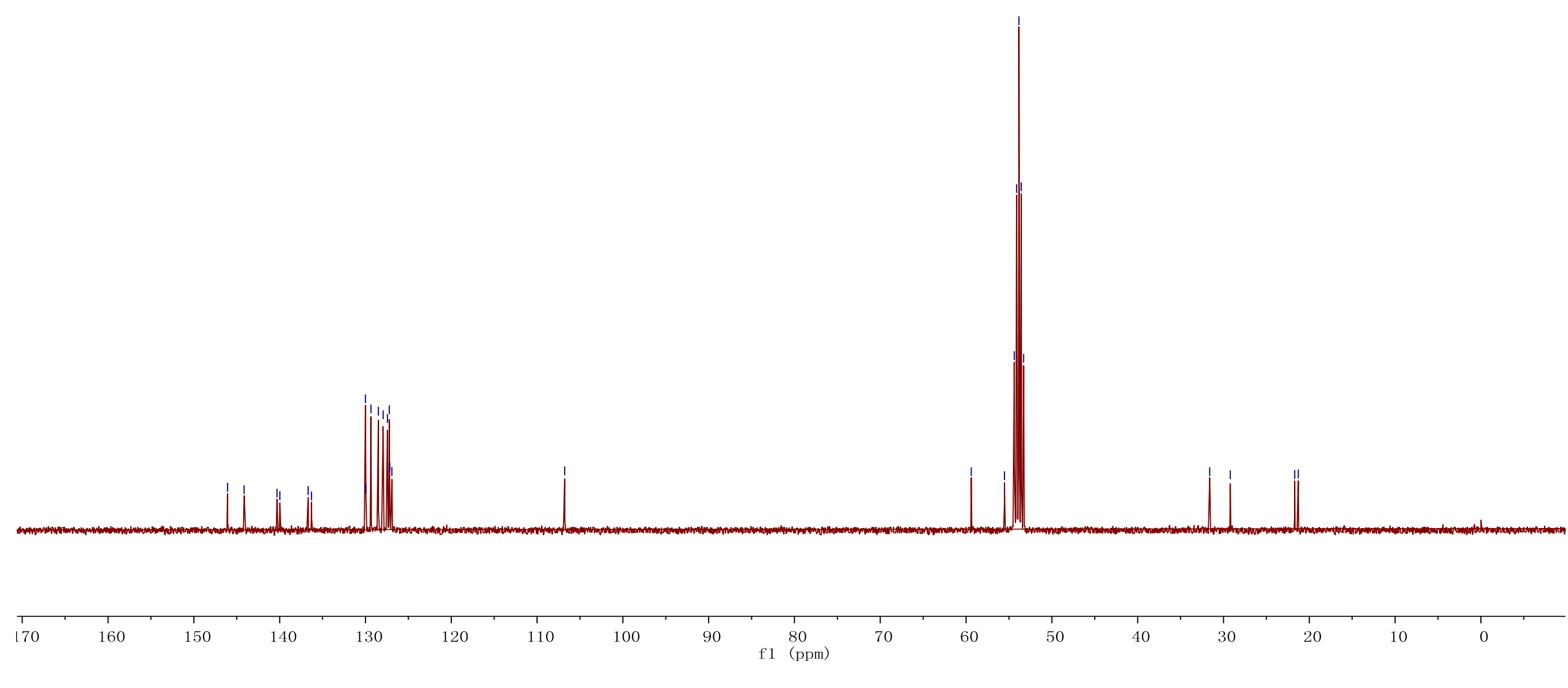




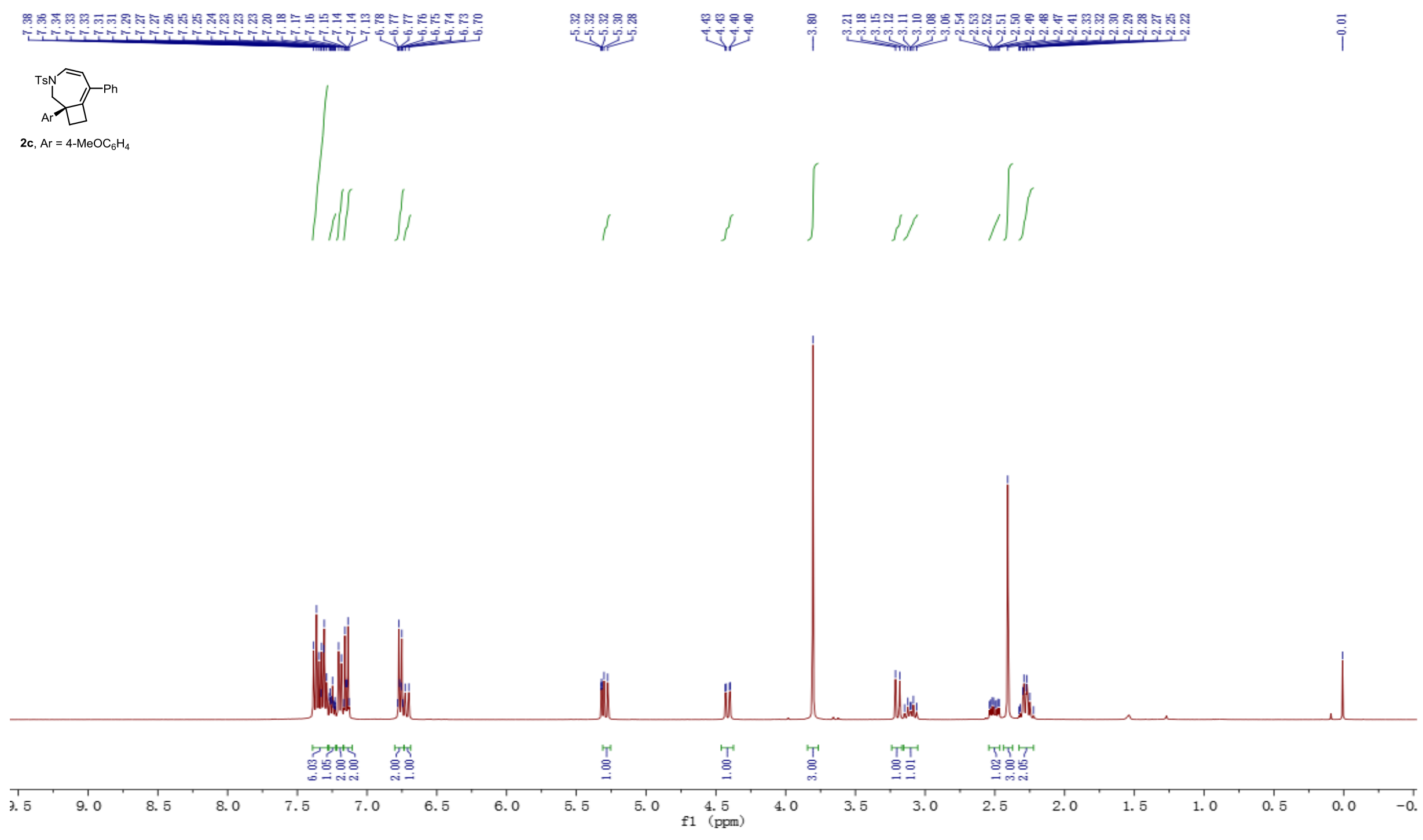




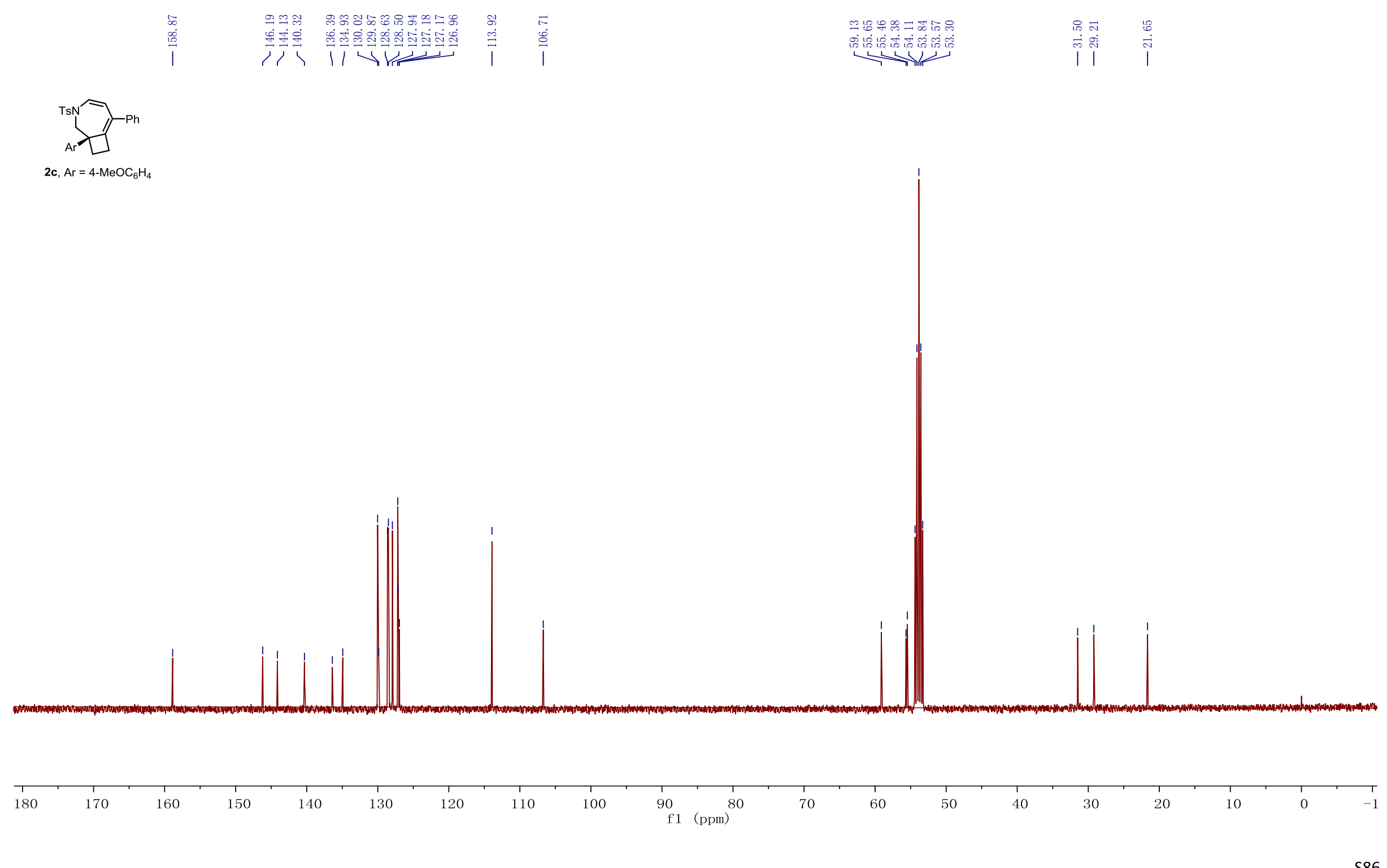




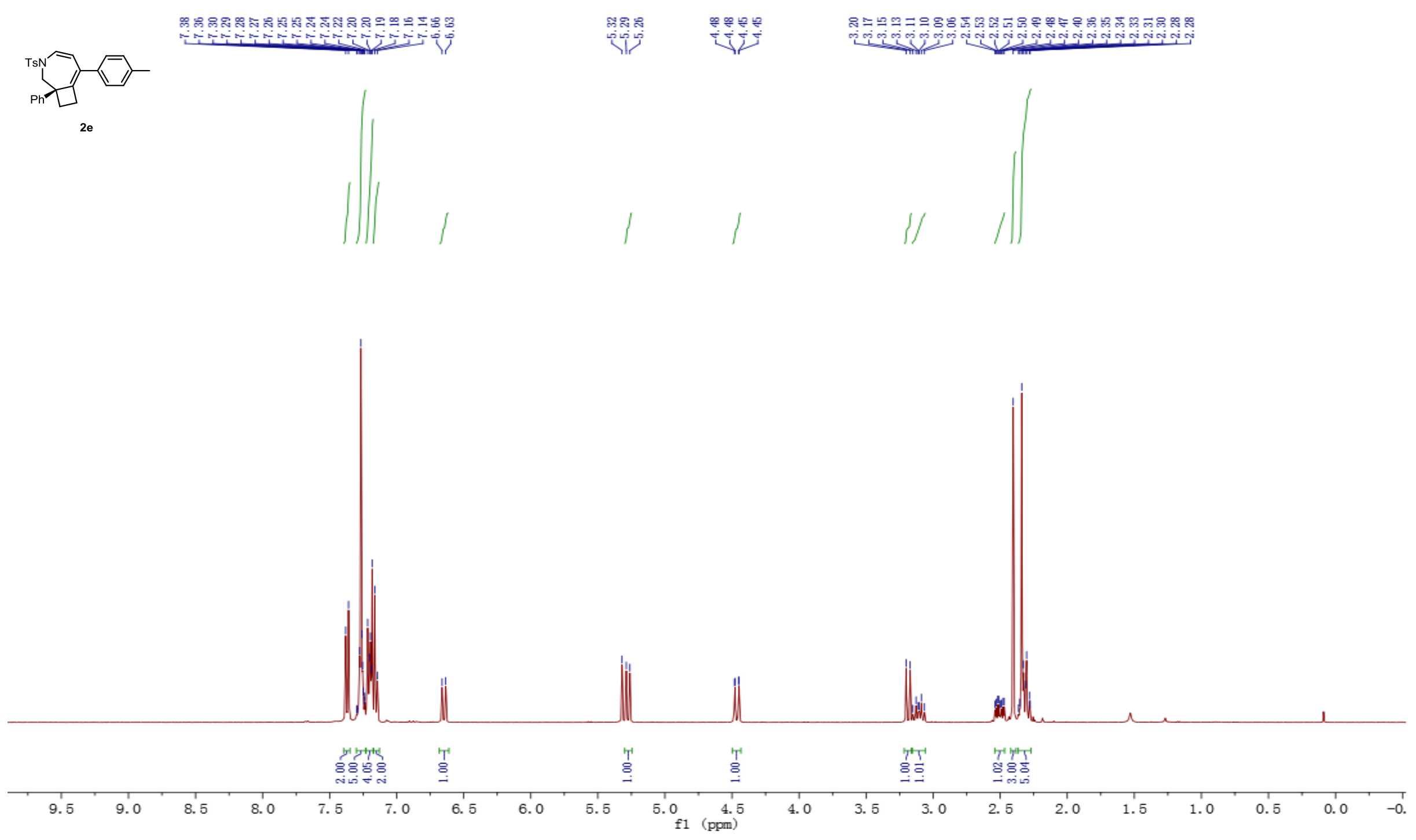




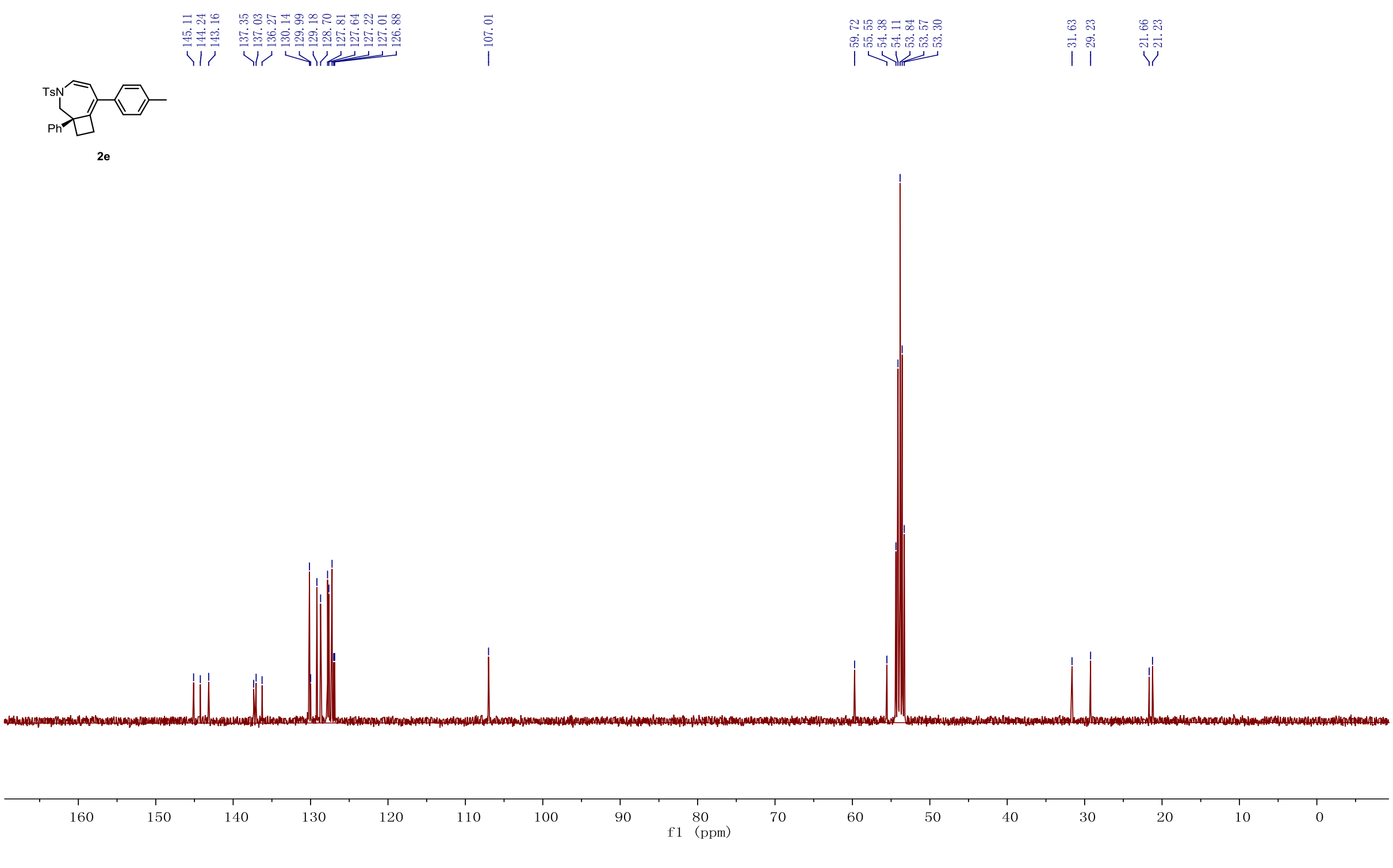




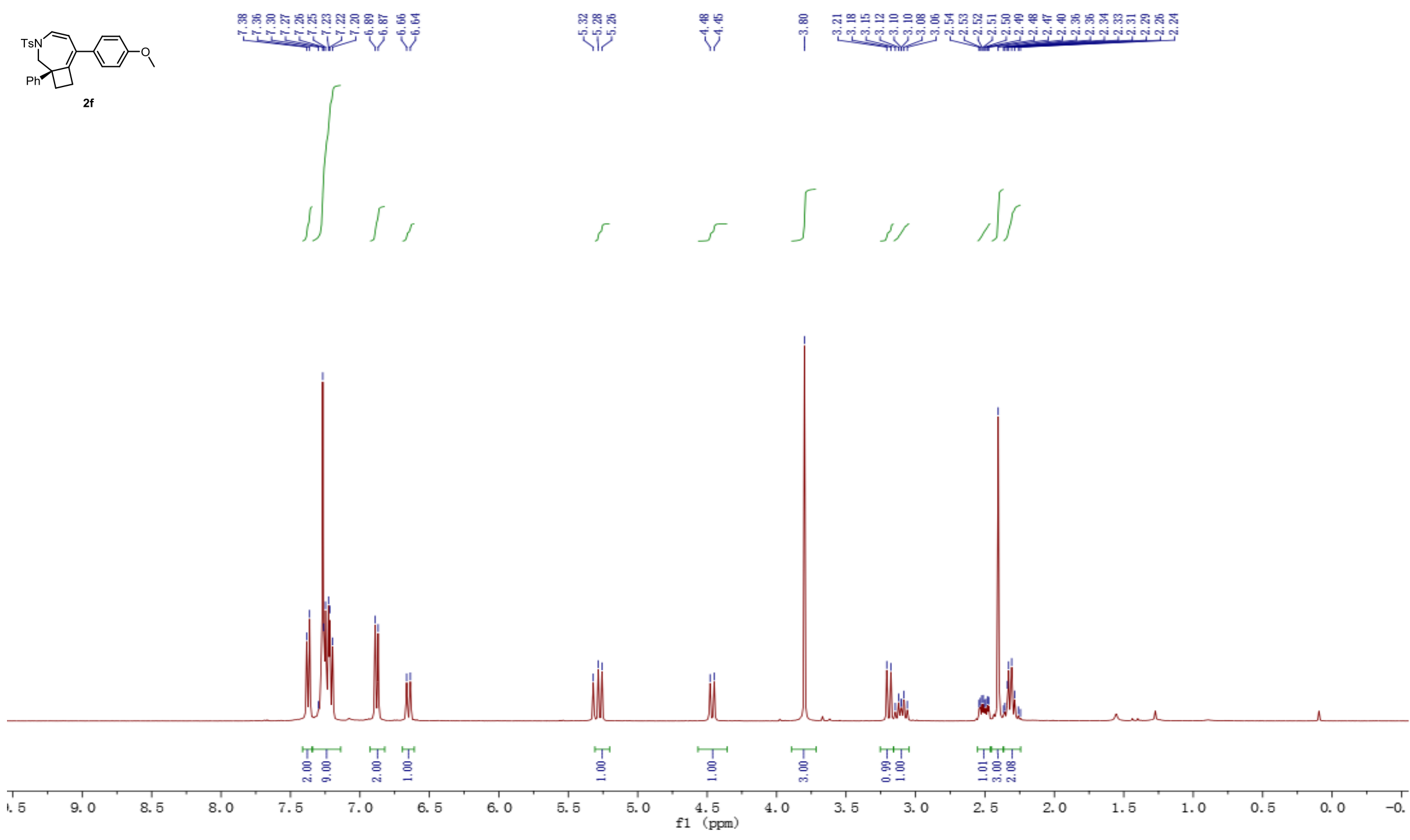




$$
\text { (1) }
$$

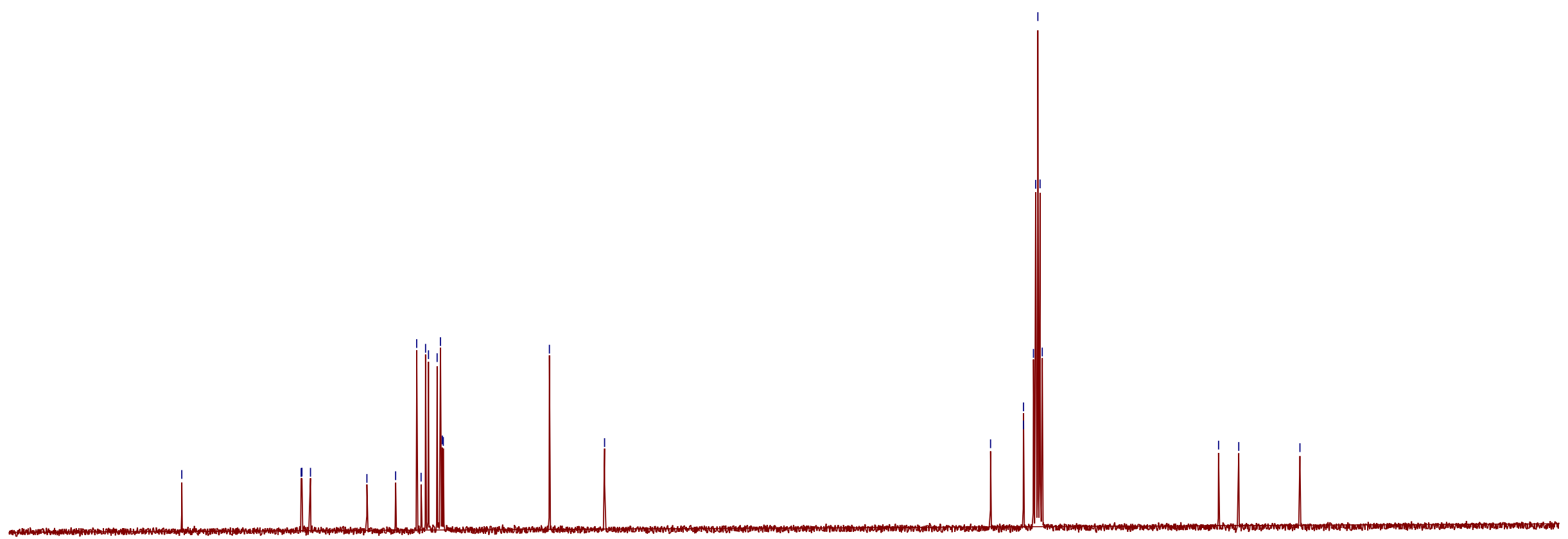

80

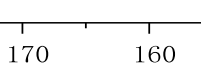

$150 \quad 140$

130

120

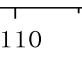

100

$90 \quad 80$

70

60

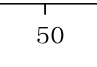

40

30

20 


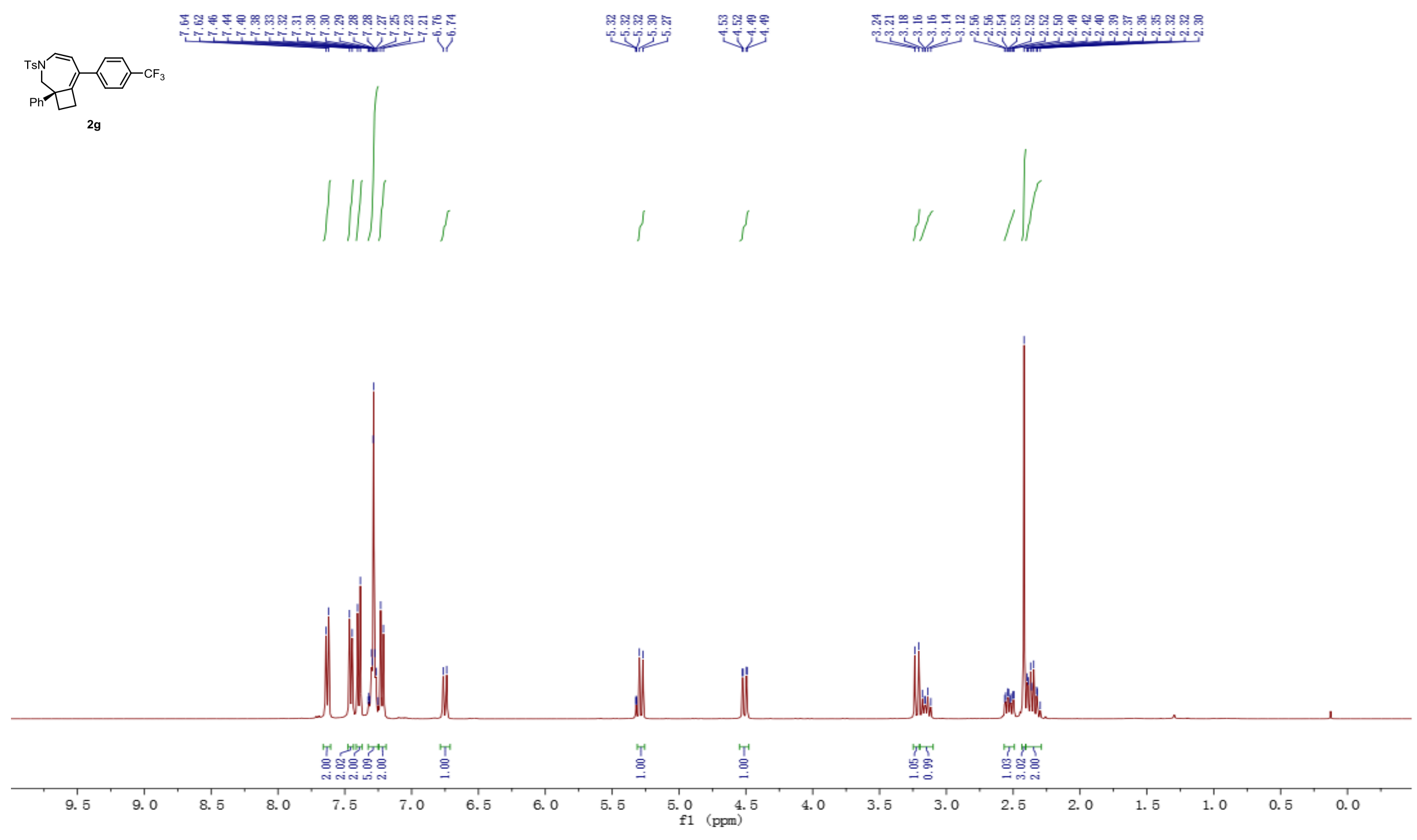




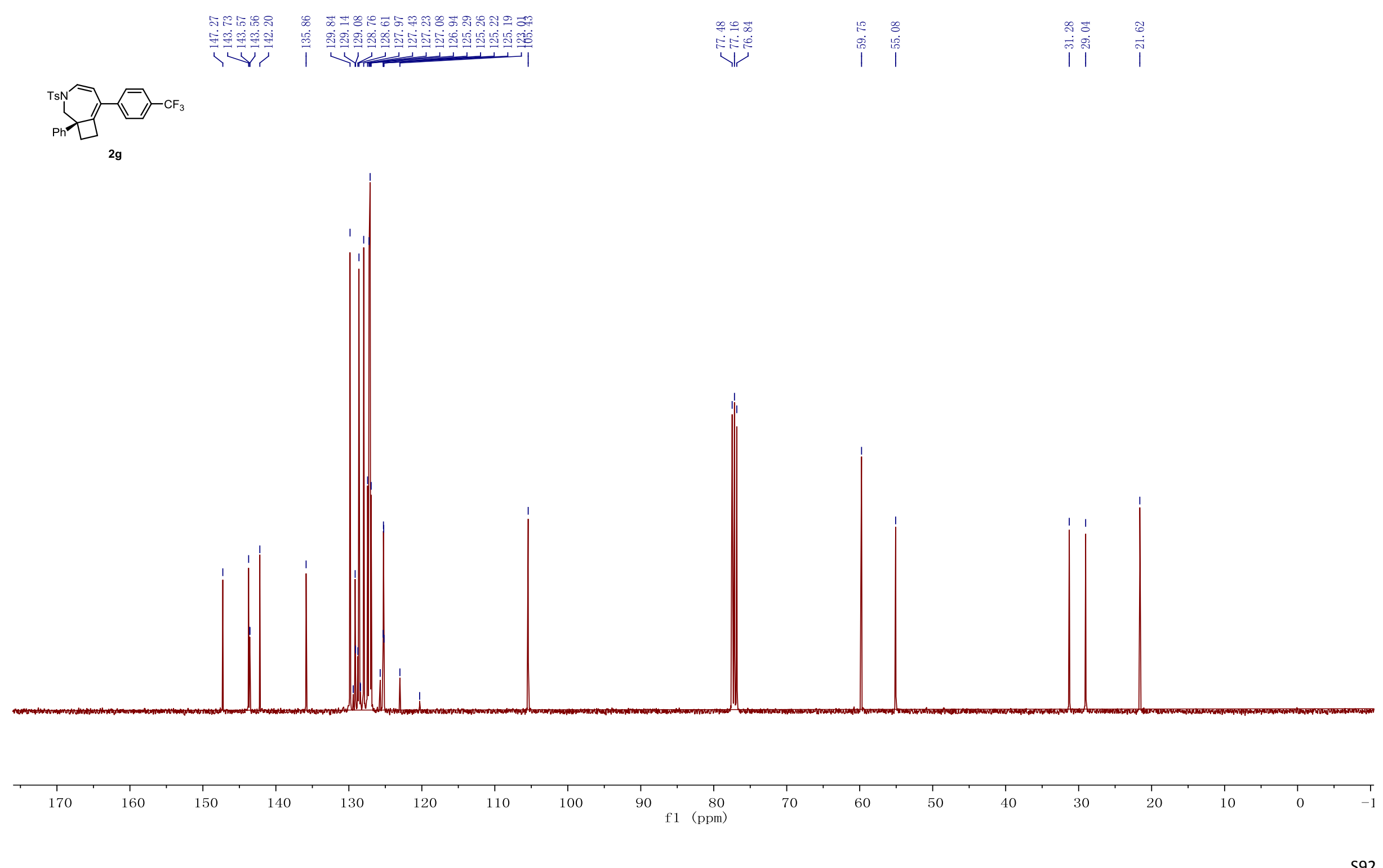




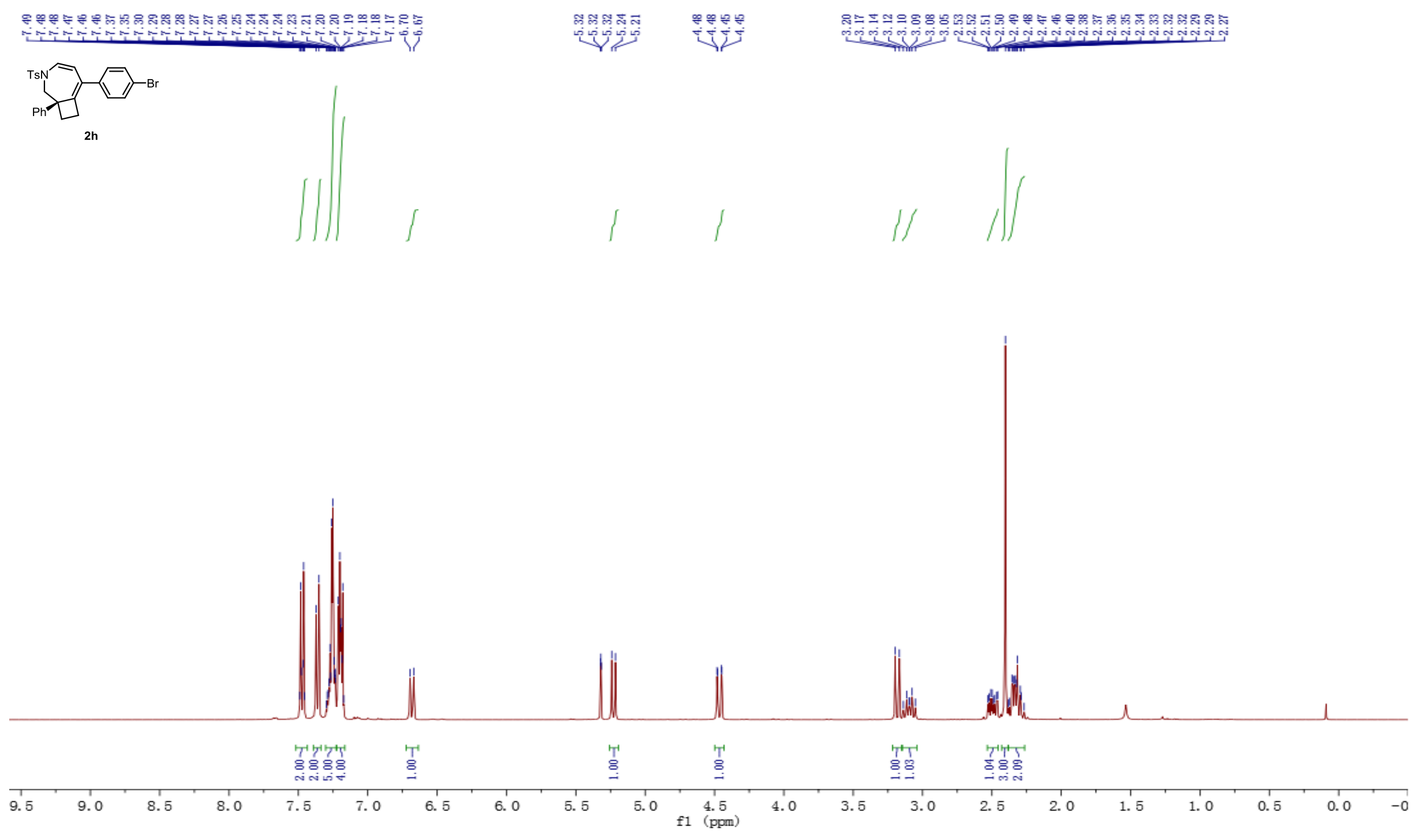





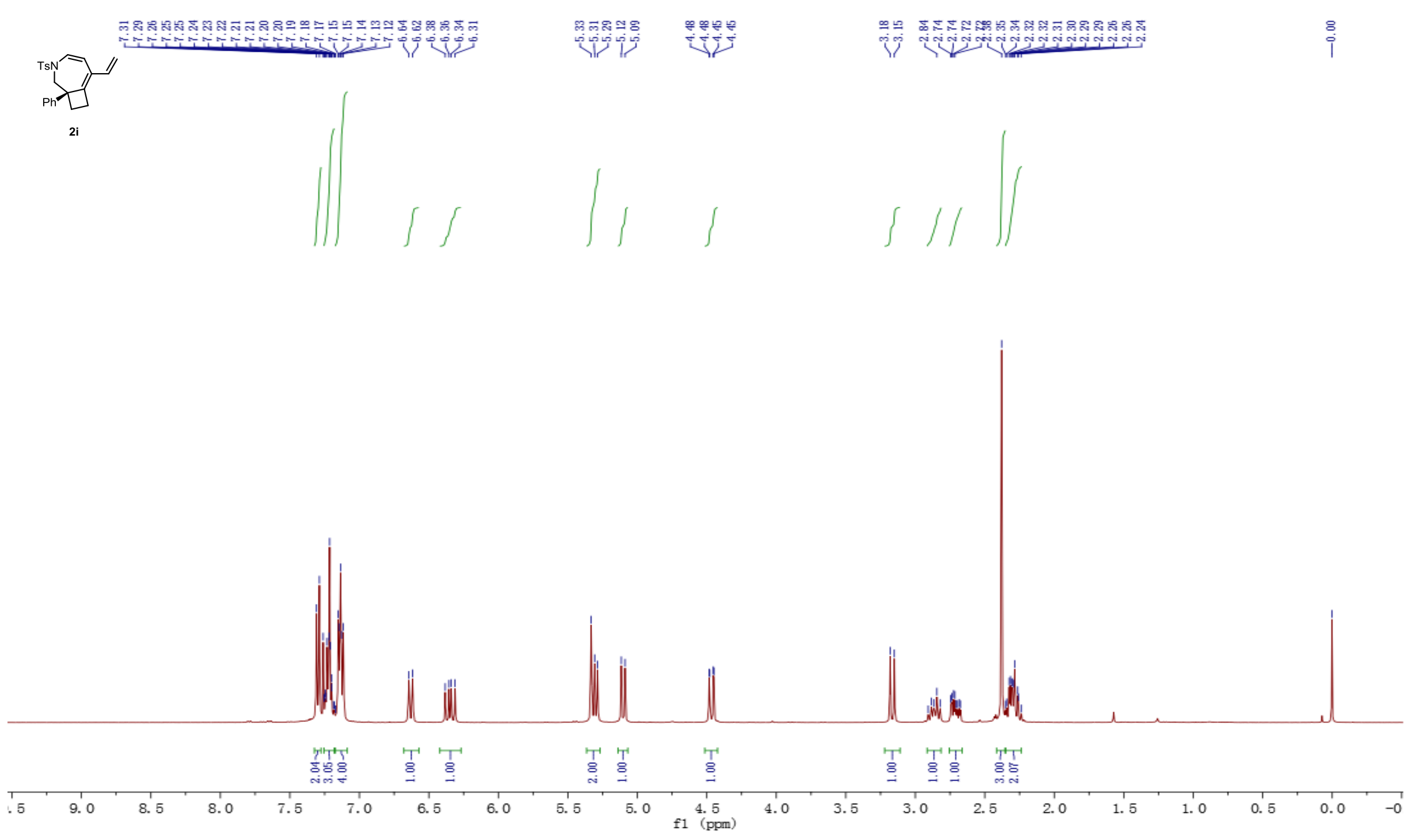




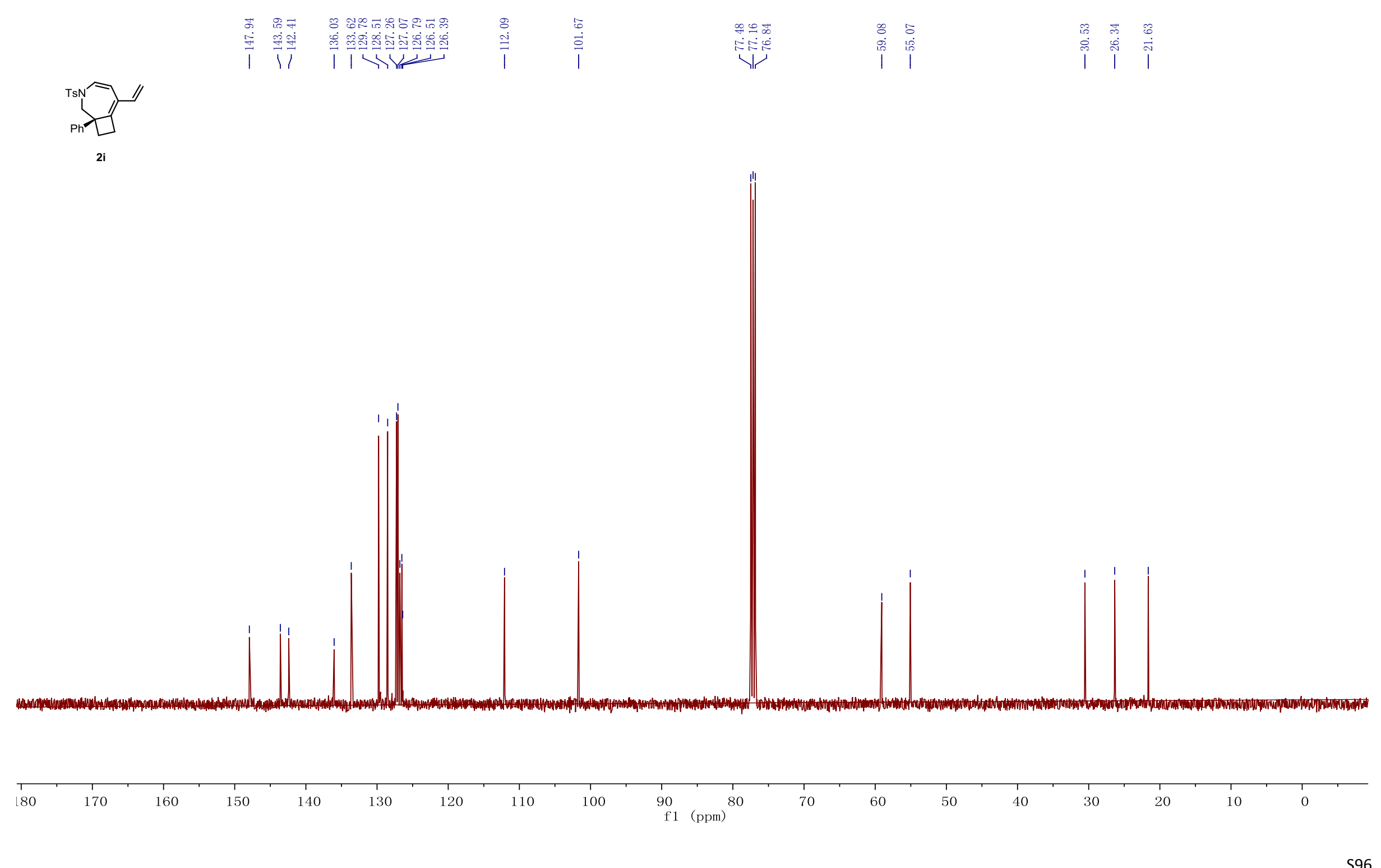




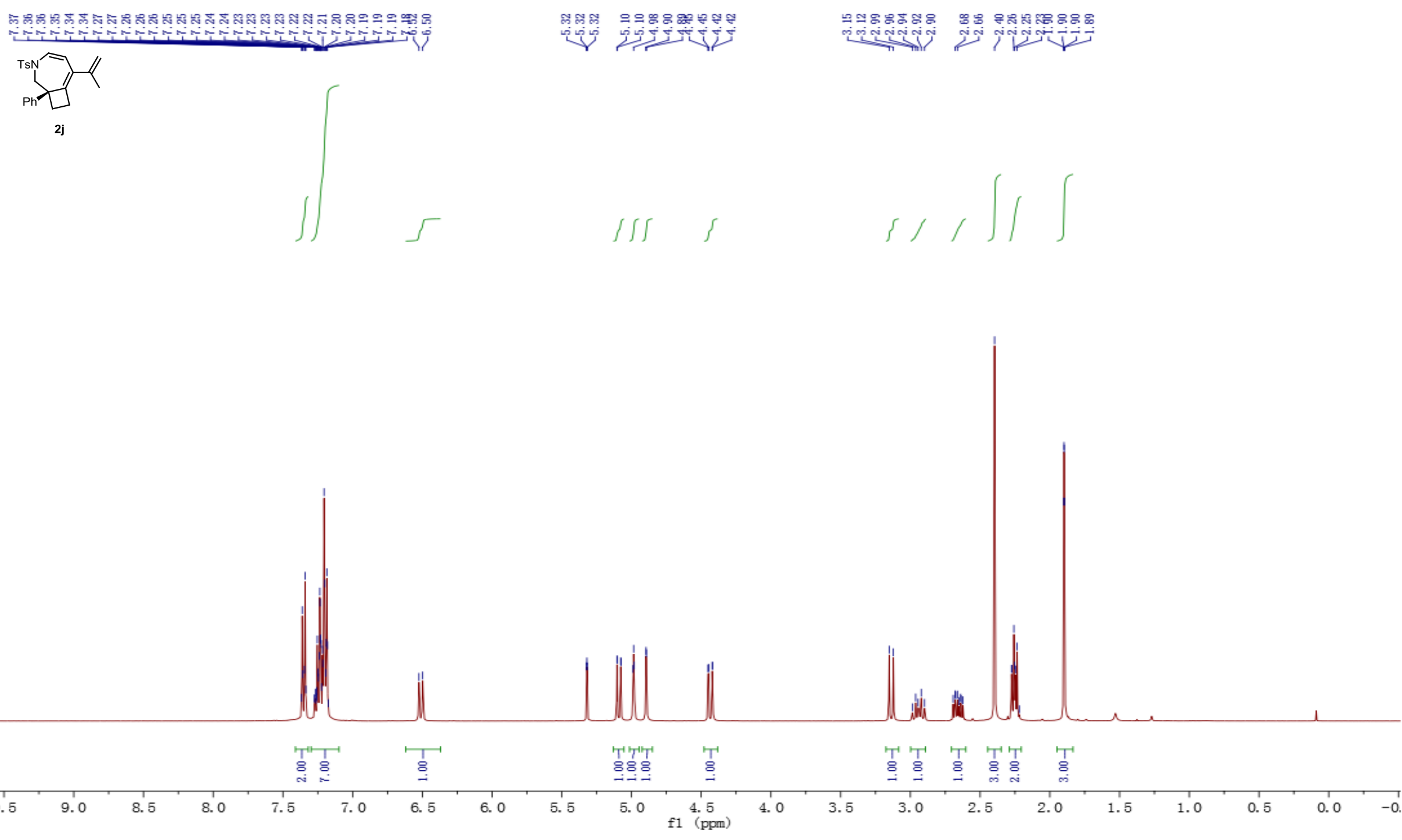



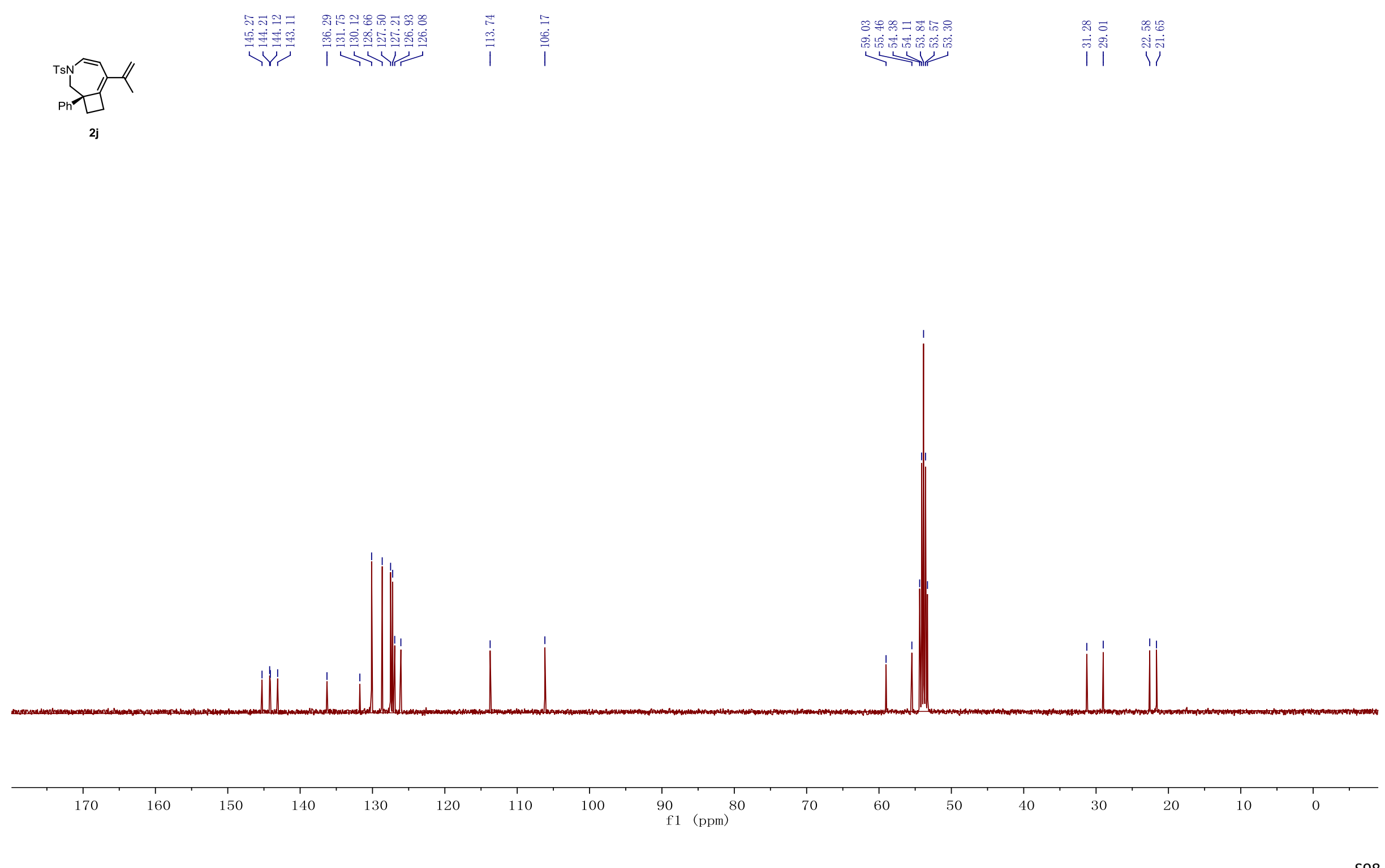


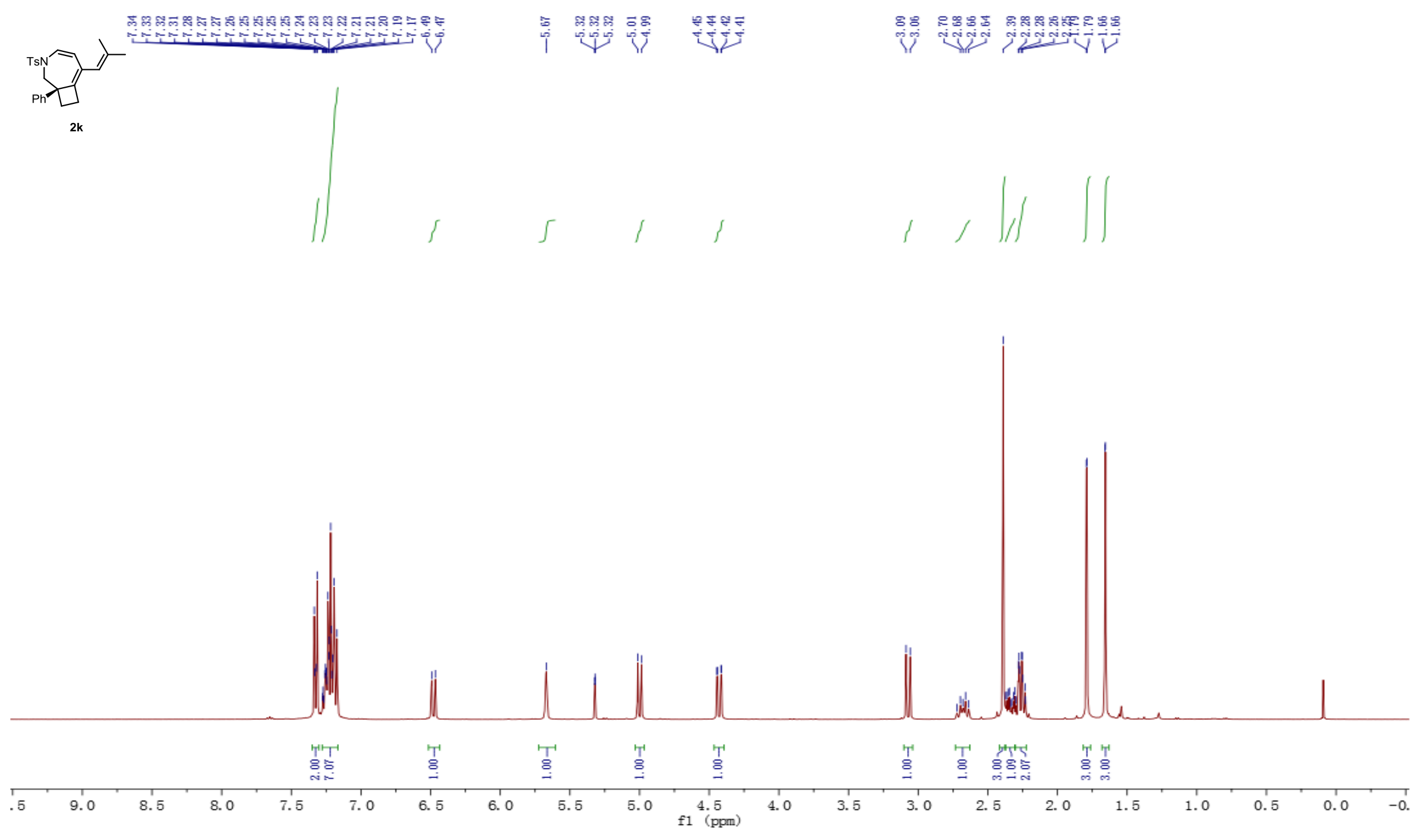




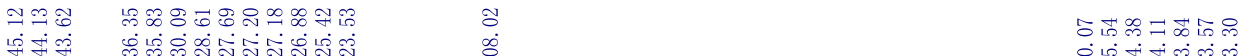

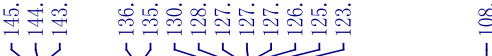

(1)

$2 \mathbf{k}$

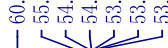

.

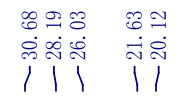

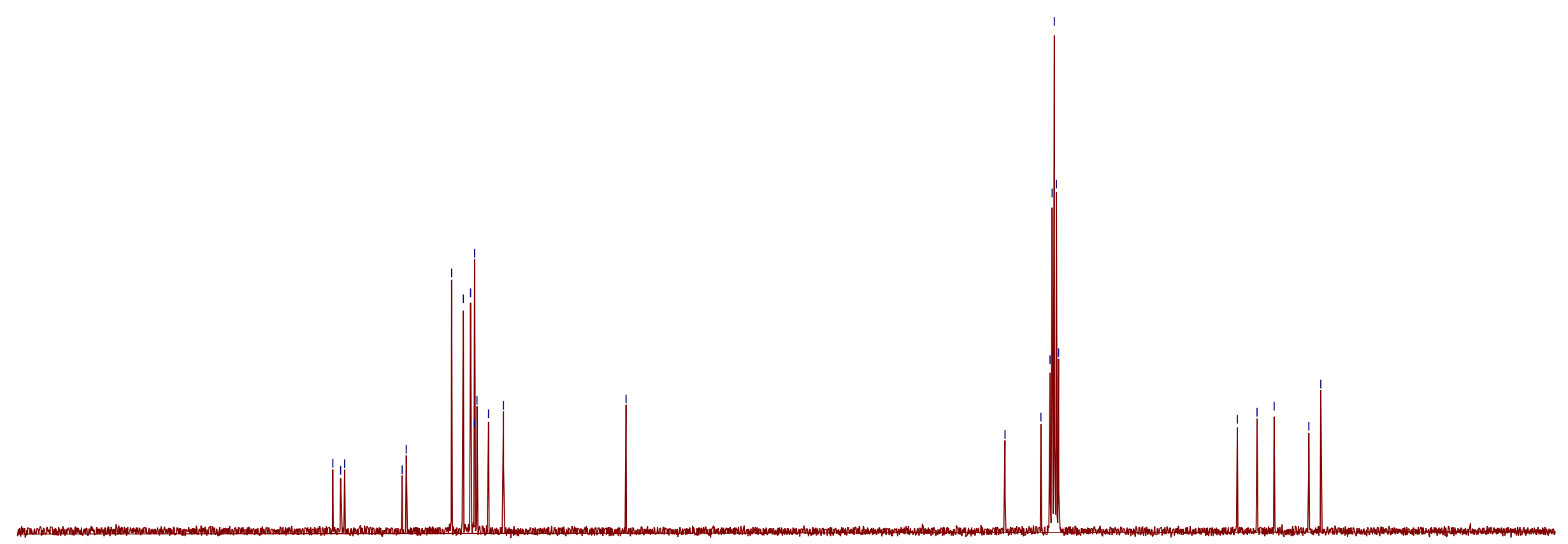

180

170

160

150

140

130

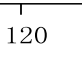

110

100

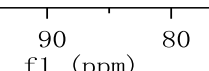

f1 (ppm)

70

60

50

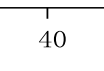

30

20 


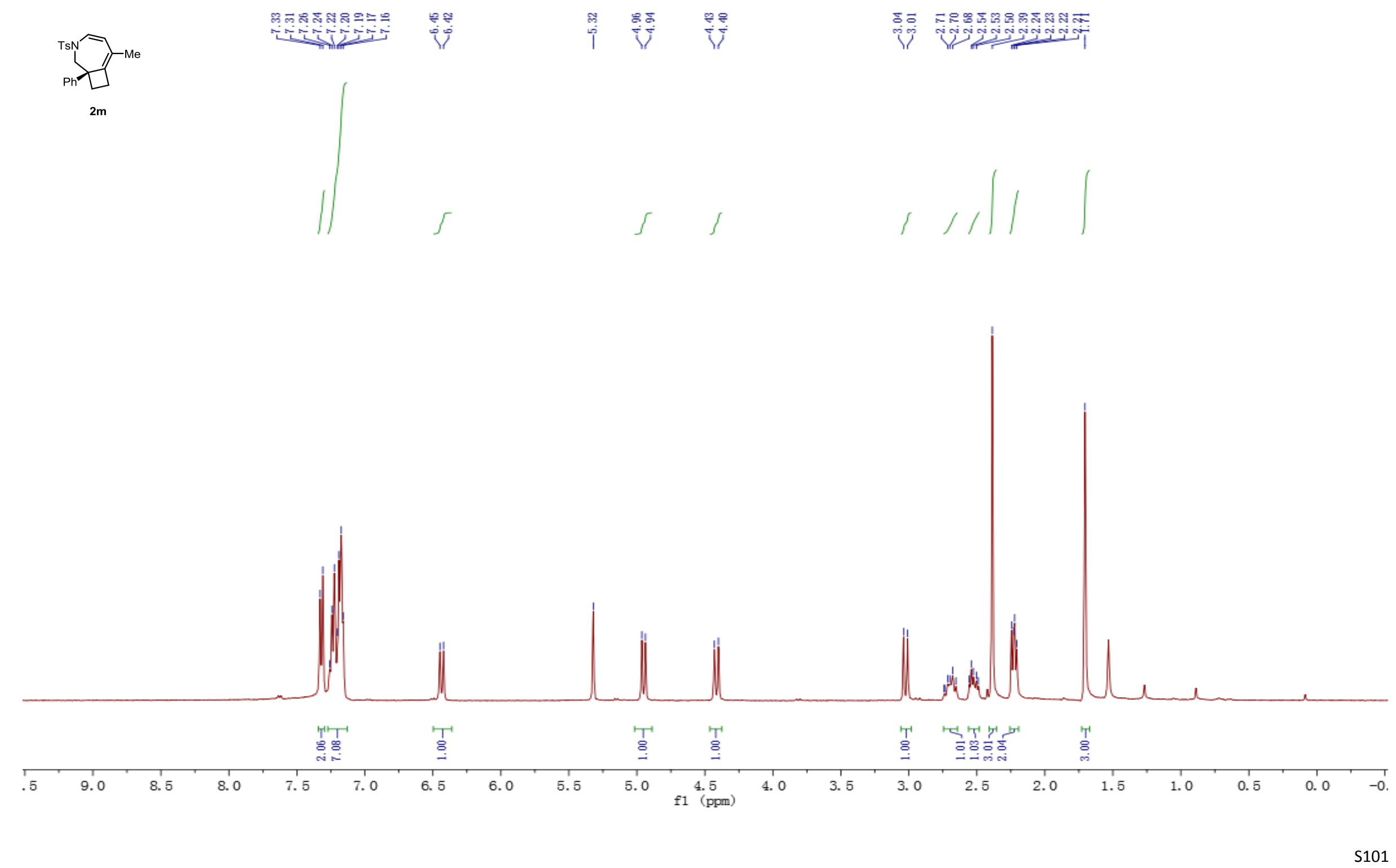




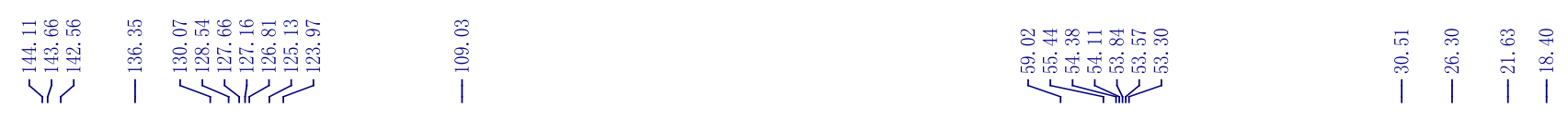

-Me

2m

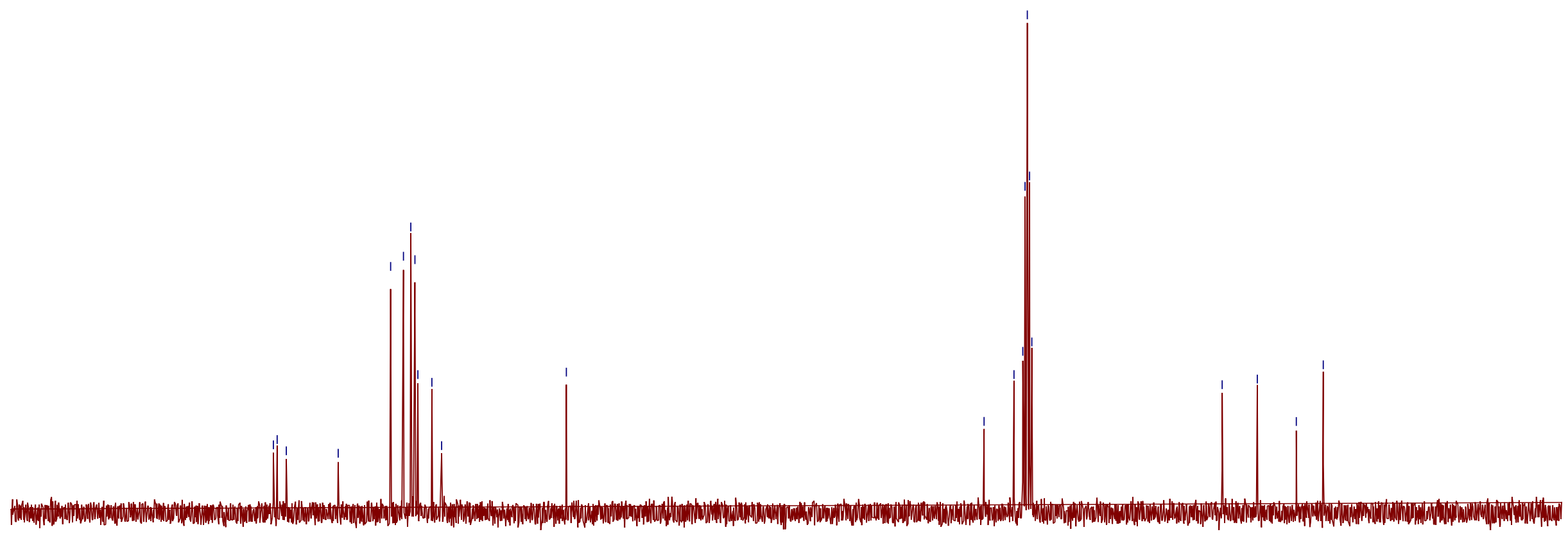




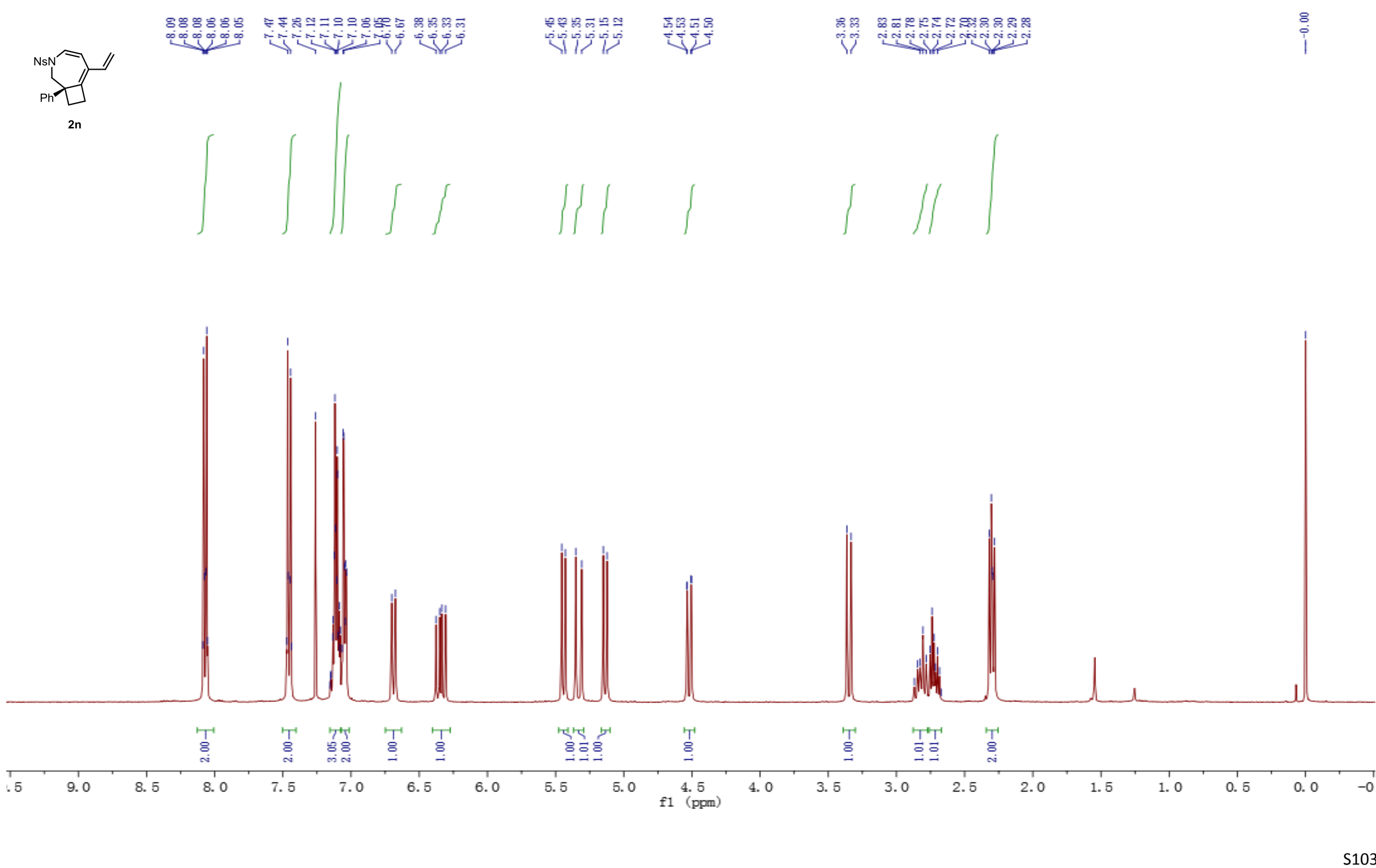




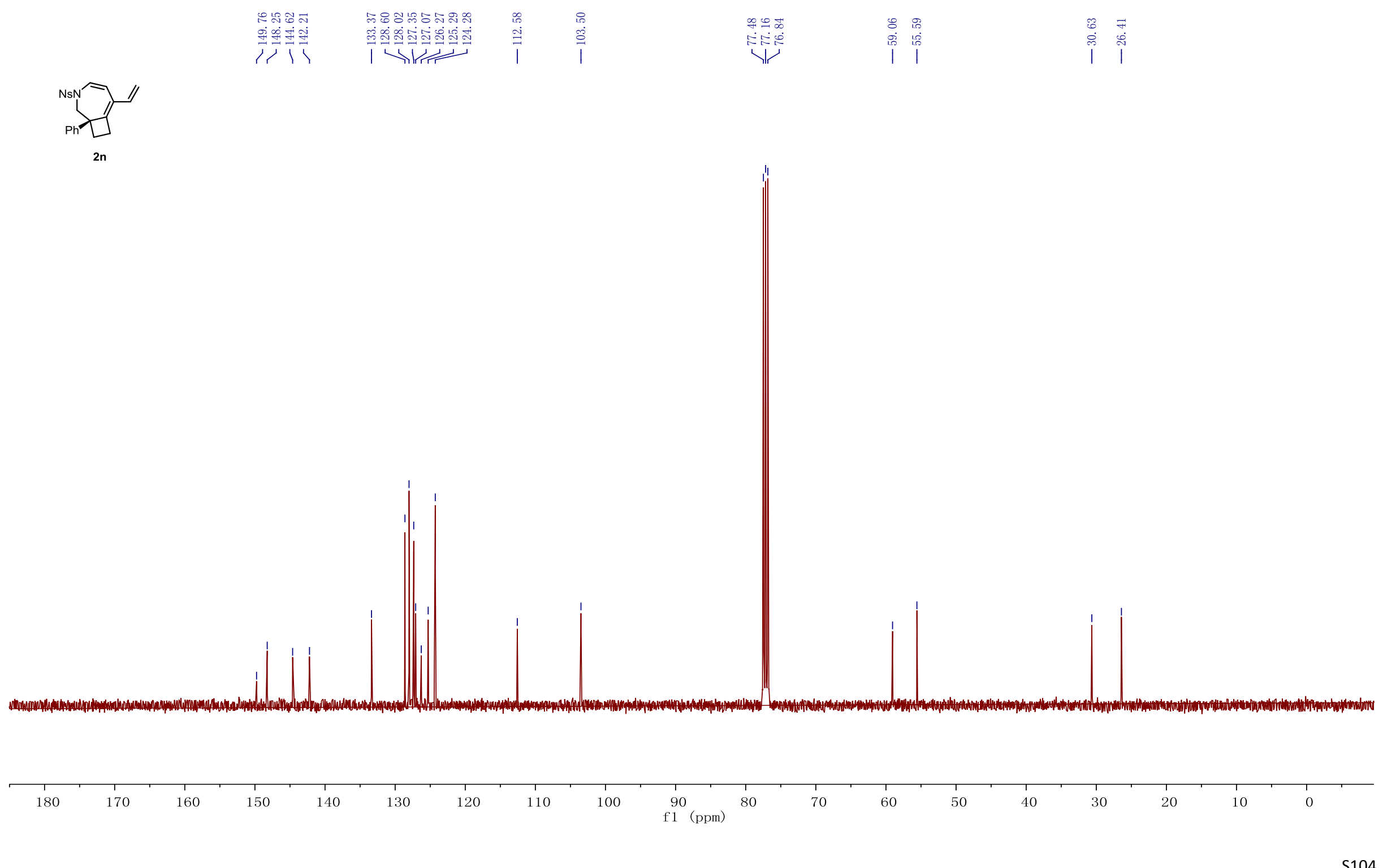




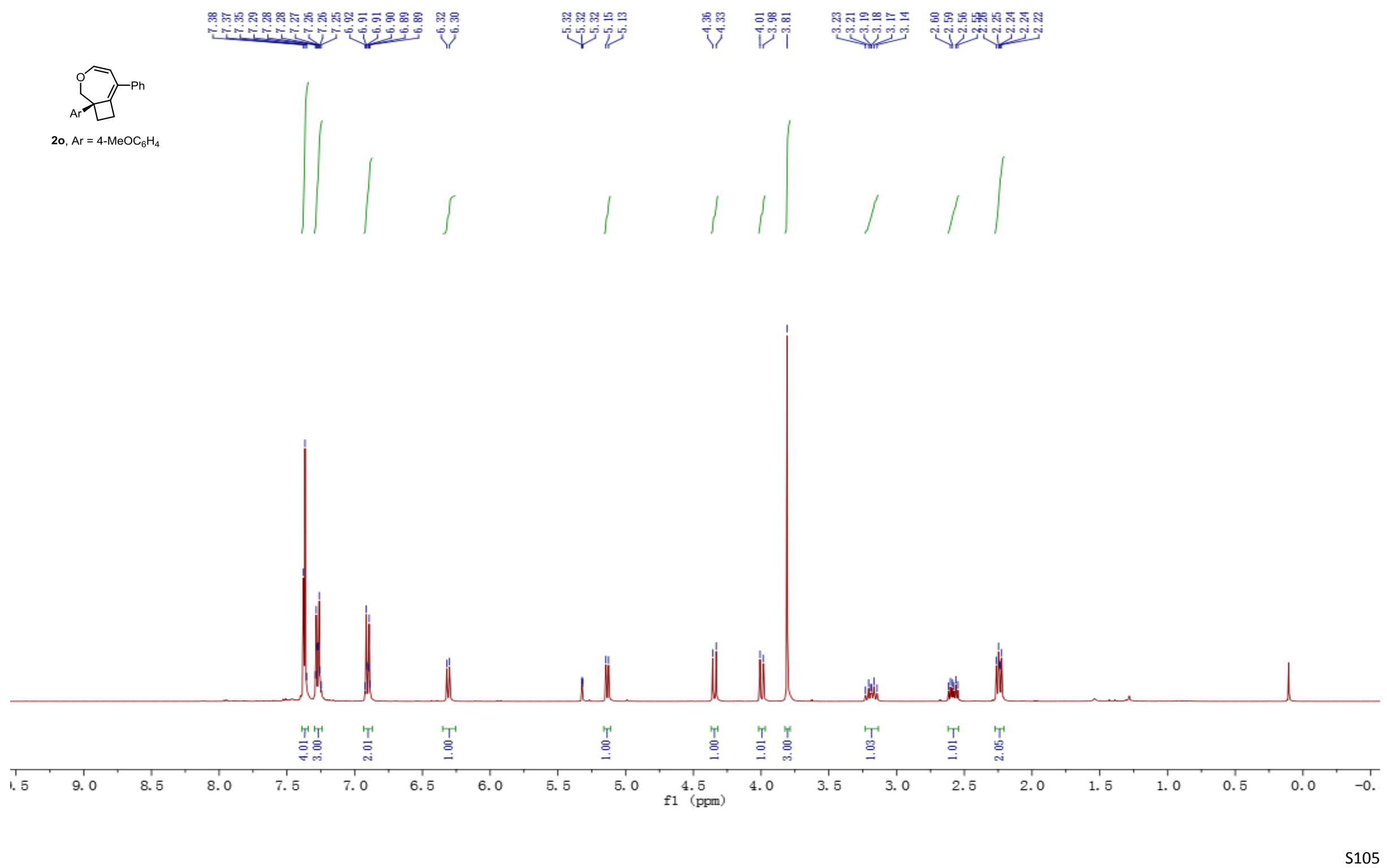




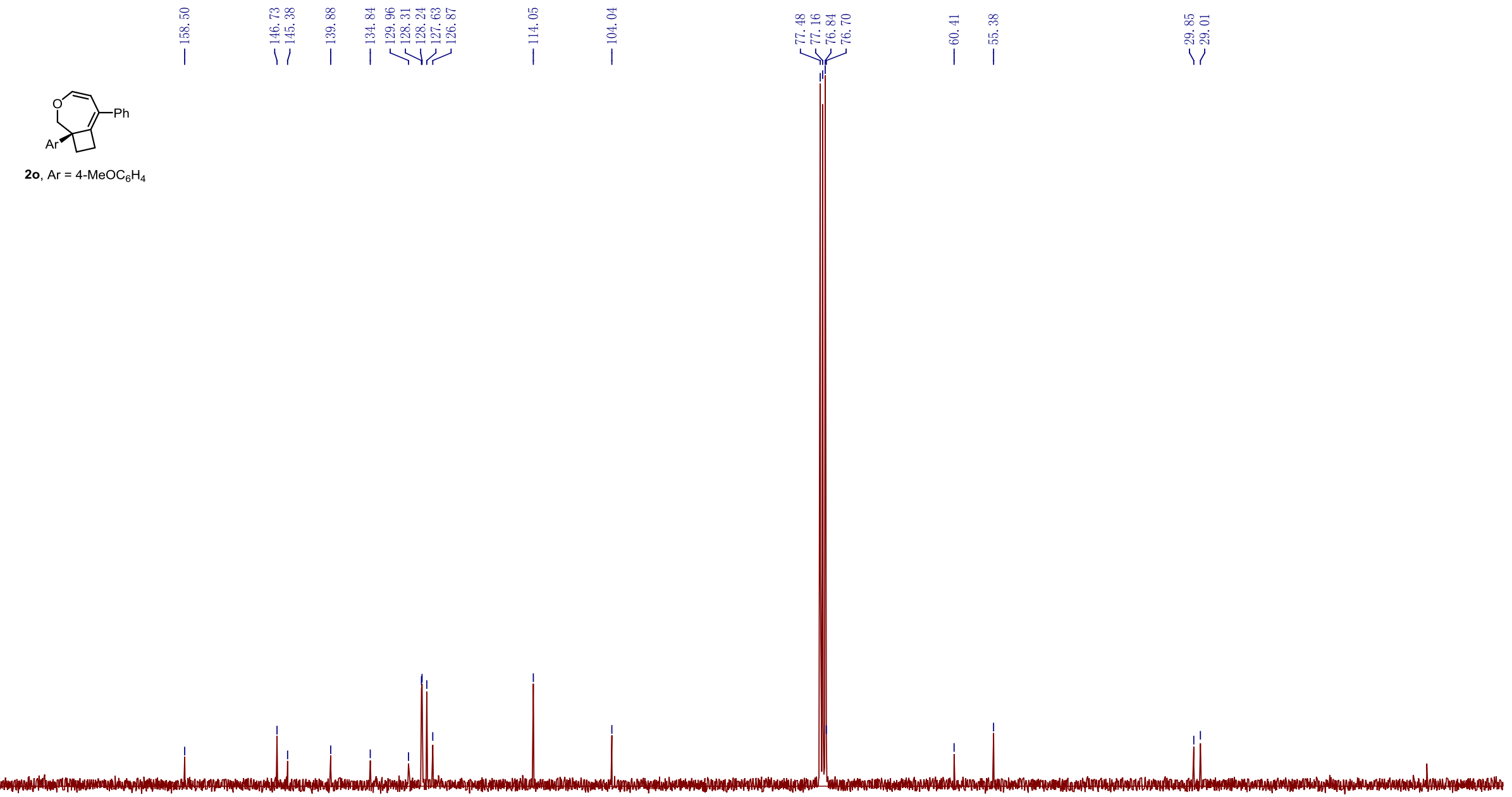

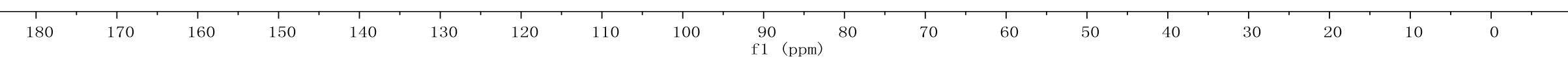




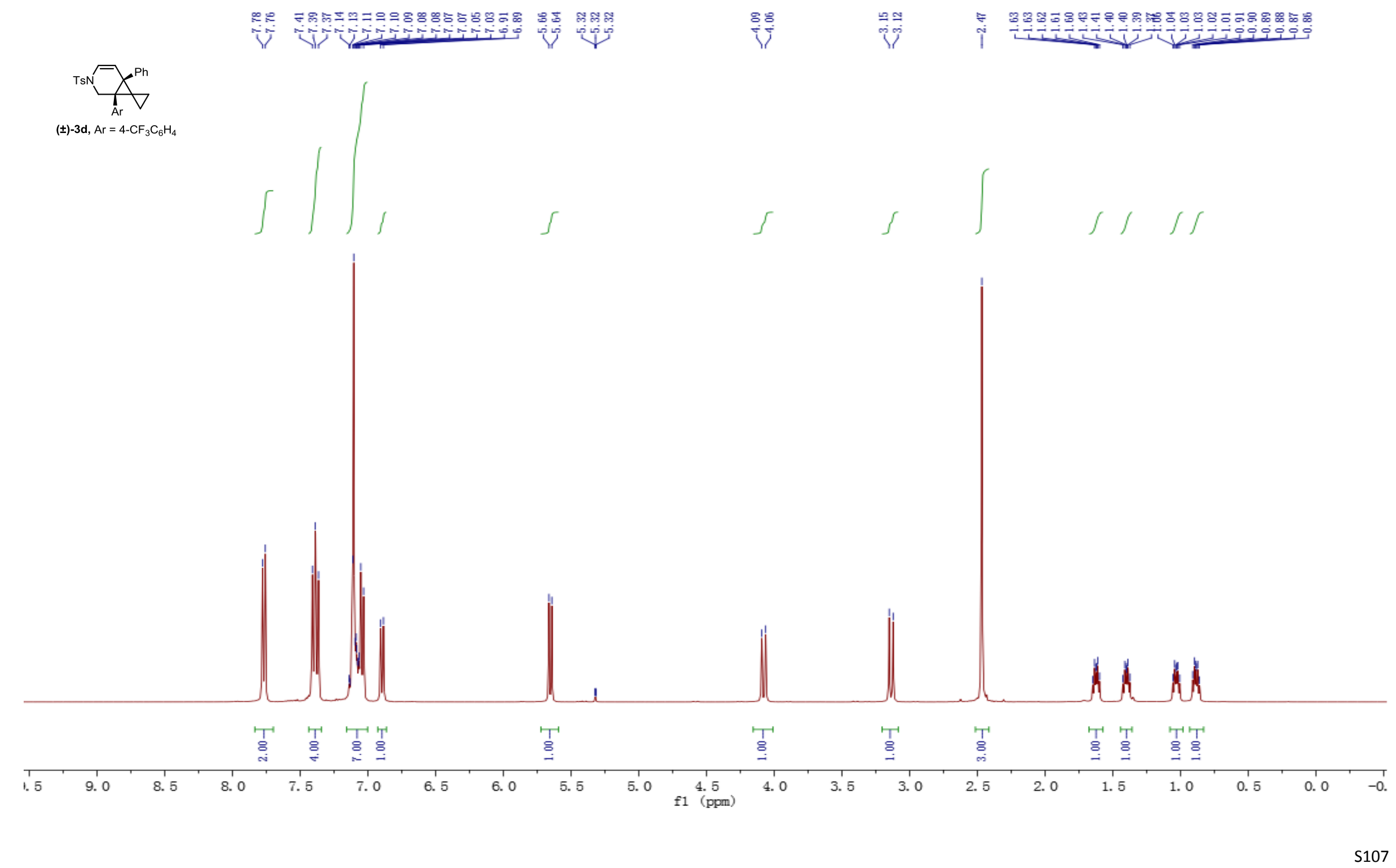




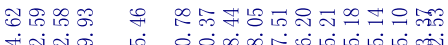

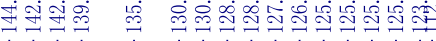

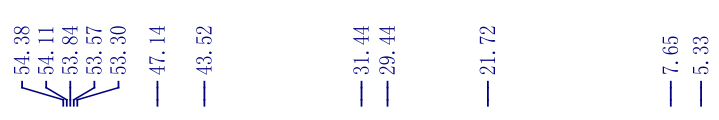

$\underbrace{P h}_{A r}$

$( \pm)-3 d, A r=4-C_{3} \mathrm{C}_{6} \mathrm{H}_{4}$

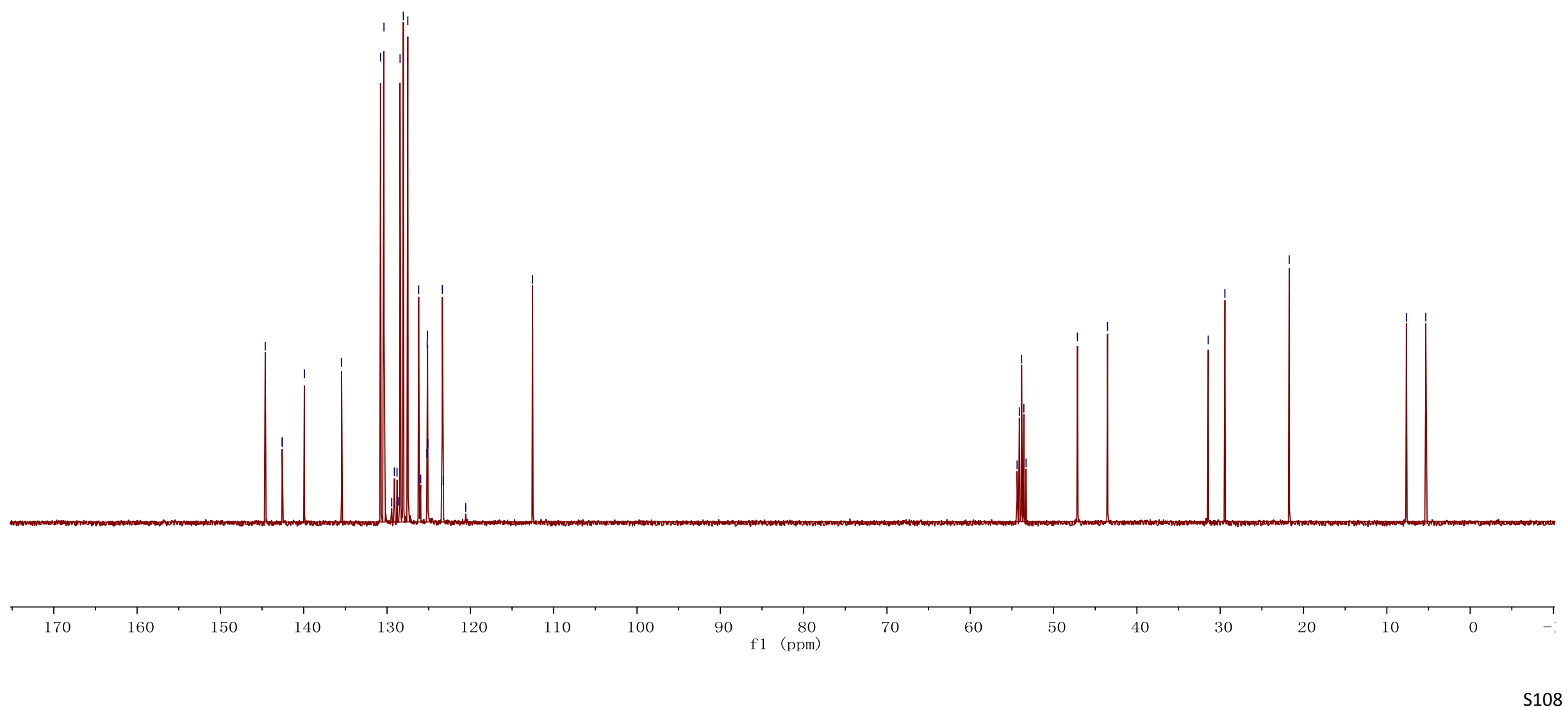




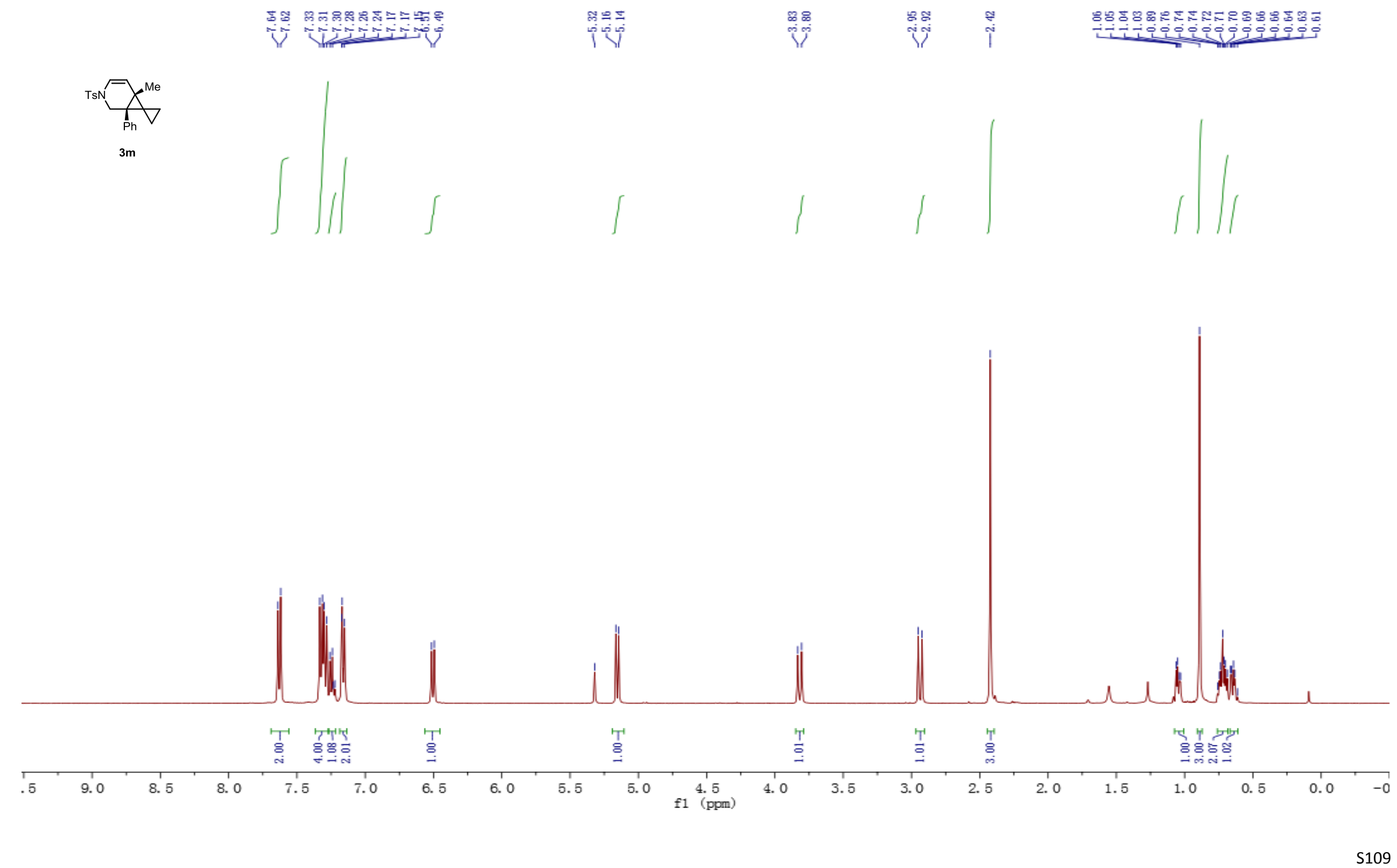


$\underbrace{\operatorname{TsN}}_{\mathrm{Ph}}$

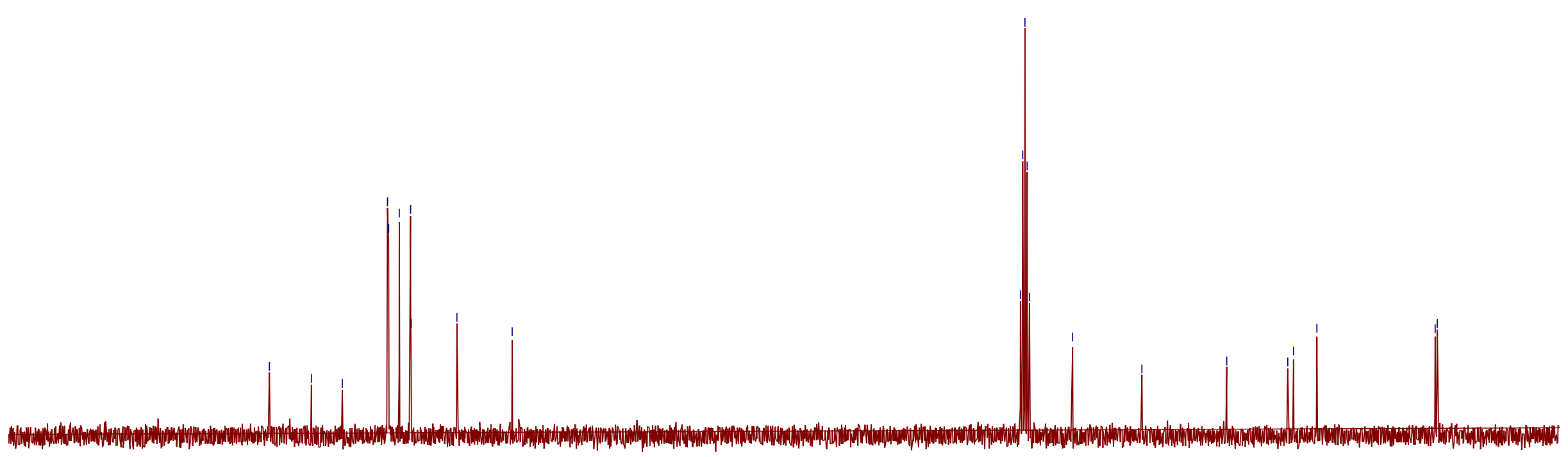


VII. High Performance Liquid Chromatography (HPLC) Data<smiles></smiles>

Chromatogram : LCL-855-r_channel1

Acquired : 2018-6-7 14:24:28

Processed : 2018-6-7 15:14:46

Printed : 2018-6-7 15:16:01

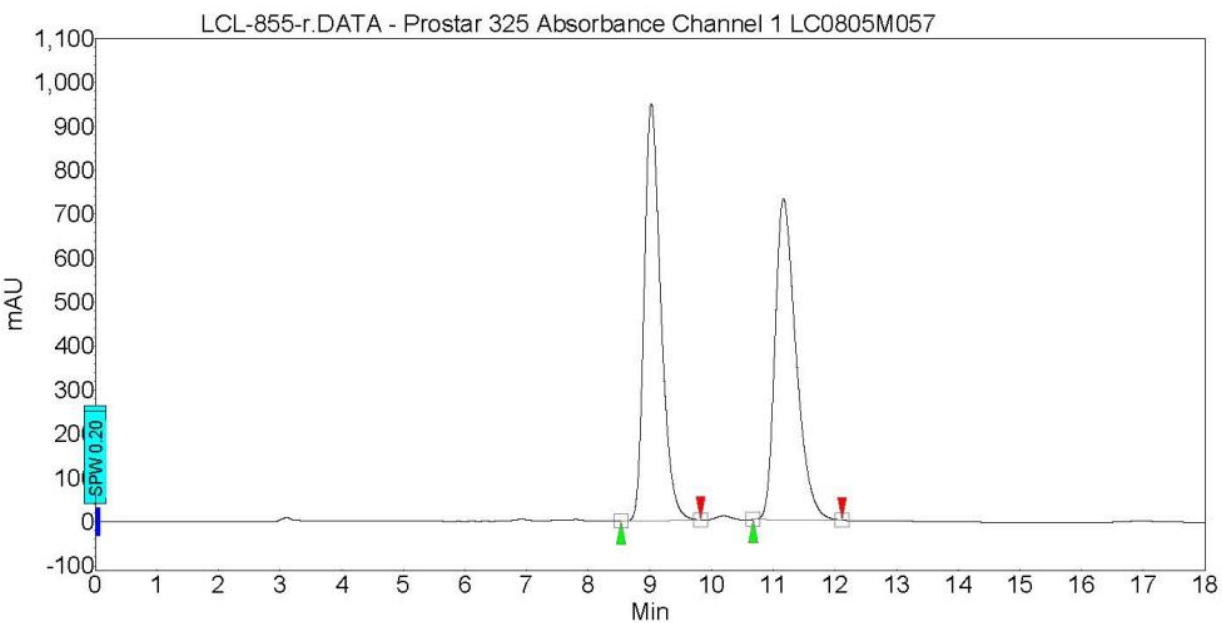

Peak results :

\begin{tabular}{|c|c|r|r|r|r|r|}
\hline Index & Name & $\begin{array}{r}\text { Time } \\
\text { [Min] }\end{array}$ & $\begin{array}{r}\text { Amount } \\
\text { [\% Areal }\end{array}$ & $\begin{array}{r}\text { Height } \\
\text { [mAU] }\end{array}$ & $\begin{array}{r}\text { Area } \\
\text { [mAU.Min] }\end{array}$ & $\begin{array}{r}\text { Area \% } \\
\text { [\%] }\end{array}$ \\
\hline \hline 1 & - & 9.02 & 50.71 & 948.7 & 296.8 & 50.706 \\
\hline 2 & - & 11.17 & 49.29 & 730.4 & 288.6 & 49.294 \\
\hline & & & & & & \\
\hline Total & & & 100.00 & 1679.1 & 585.4 & 100.000 \\
\hline
\end{tabular}




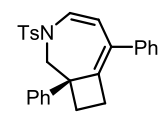

2a

\section{Chromatogram : cf-LCL-855-a_channel1}

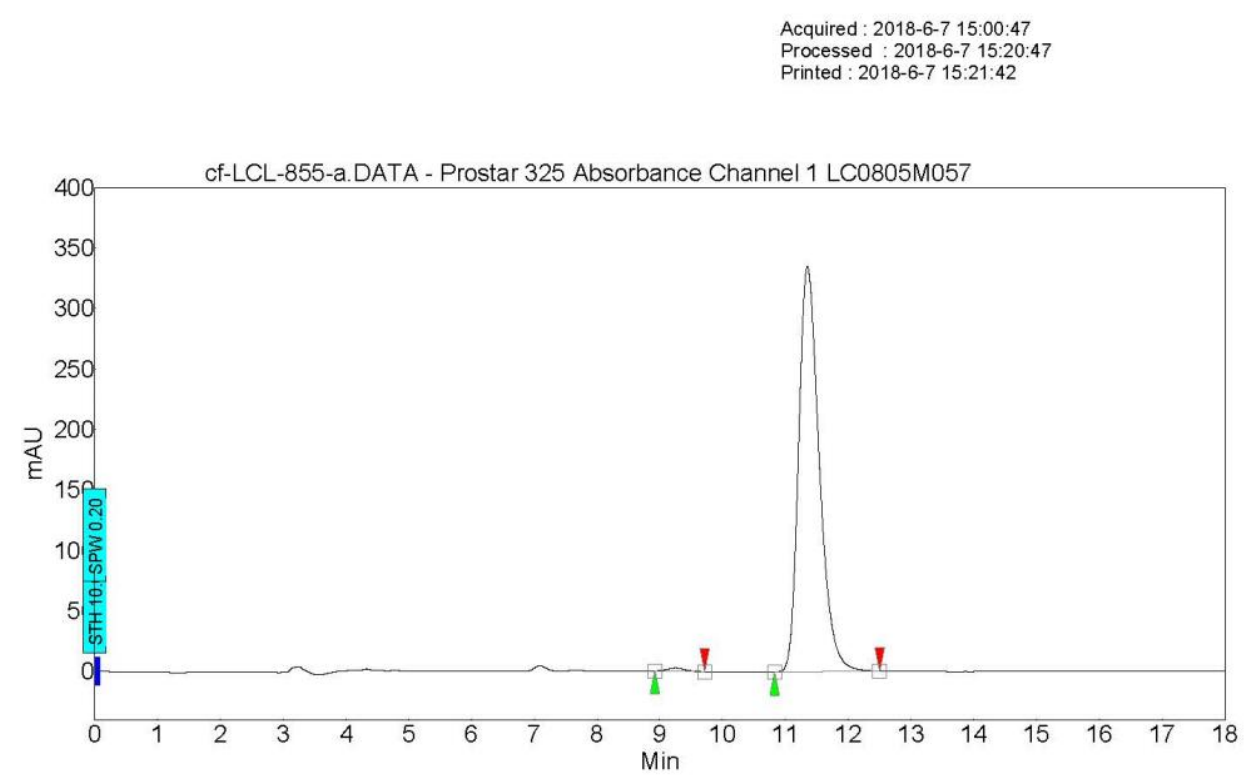

Peak results :

\begin{tabular}{|c|r|r|r|r|r|r|}
\hline Index & Name & $\begin{array}{r}\text { Time } \\
\text { [Min] }\end{array}$ & $\begin{array}{r}\text { Amount } \\
\text { [\% Area] }\end{array}$ & $\begin{array}{r}\text { Height } \\
\text { [mAU] }\end{array}$ & $\begin{array}{r}\text { Area } \\
\text { [mAU.Min] }\end{array}$ & $\begin{array}{r}\text { Area \% } \\
\text { [\%] }\end{array}$ \\
\hline \hline 2 & - & 9.26 & 0.68 & 2.7 & 0.9 & 0.683 \\
\hline 1 & - & 11.35 & 99.32 & 335.0 & 127.6 & 99.317 \\
\hline & & & & & & \\
\hline Total & & & 100.00 & 337.7 & 128.5 & 100.000 \\
\hline
\end{tabular}




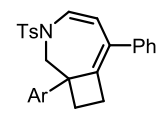

(士)-2b, $\mathrm{Ar}=4-\mathrm{MeC}_{6} \mathrm{H}_{4}$

\section{Chromatogram : LCL-856-r_channel1}

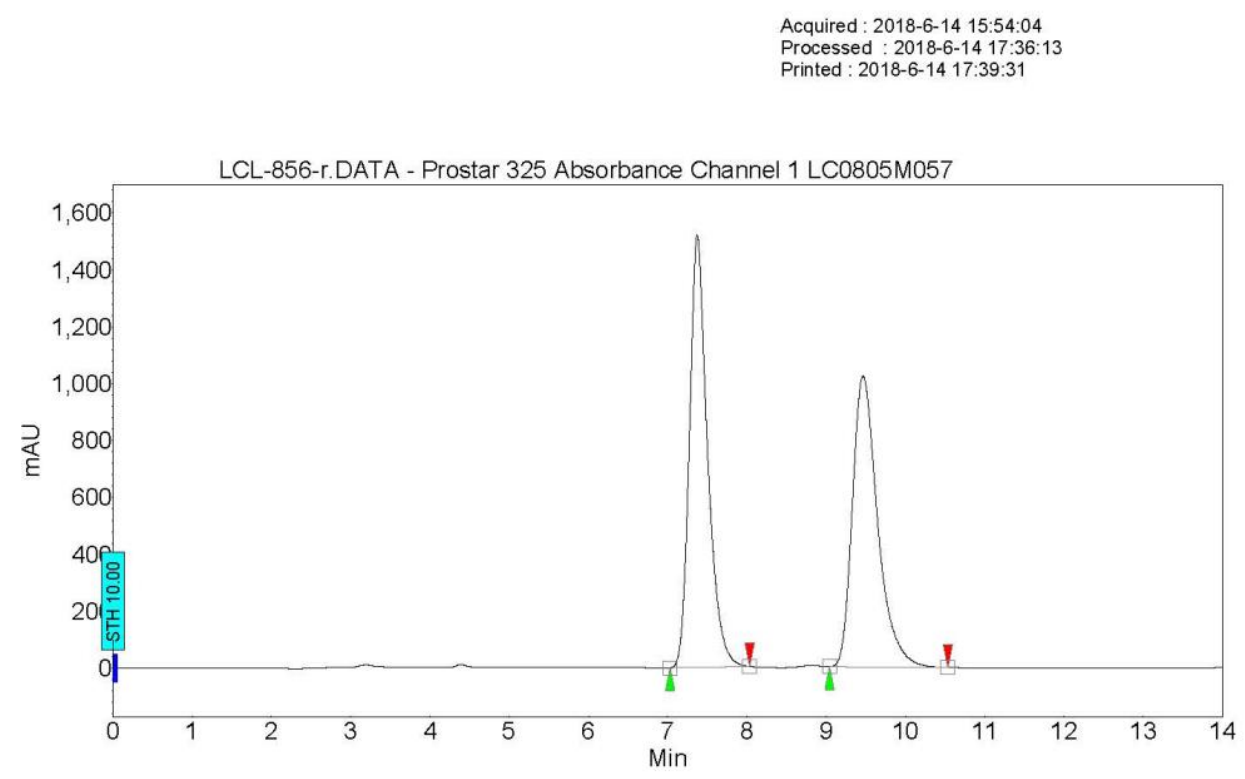

Peak results :

\begin{tabular}{|c|r|r|r|r|r|r|}
\hline Index & Name & $\begin{array}{r}\text { Time } \\
\text { [Min] }\end{array}$ & $\begin{array}{r}\text { Amount } \\
{[\% \text { Area] }}\end{array}$ & $\begin{array}{r}\text { Height } \\
\text { [mAU] }\end{array}$ & $\begin{array}{r}\text { Area } \\
\text { [mAU.Min] }\end{array}$ & $\begin{array}{r}\text { Area \% } \\
{[\%]}\end{array}$ \\
\hline \hline 1 & - & 7.37 & 51.50 & 1520.7 & 401.2 & 51.497 \\
\hline 2 & - & 9.47 & 48.50 & 1023.1 & 377.8 & 48.503 \\
\hline & & & & & & \\
\hline Total & & & 100.00 & 2543.8 & 779.0 & 100.000 \\
\hline
\end{tabular}


Dh

2b, $\mathrm{Ar}=4-\mathrm{MeC}_{6} \mathrm{H}_{4}$

Chromatogram : LCL-856-a_channel1

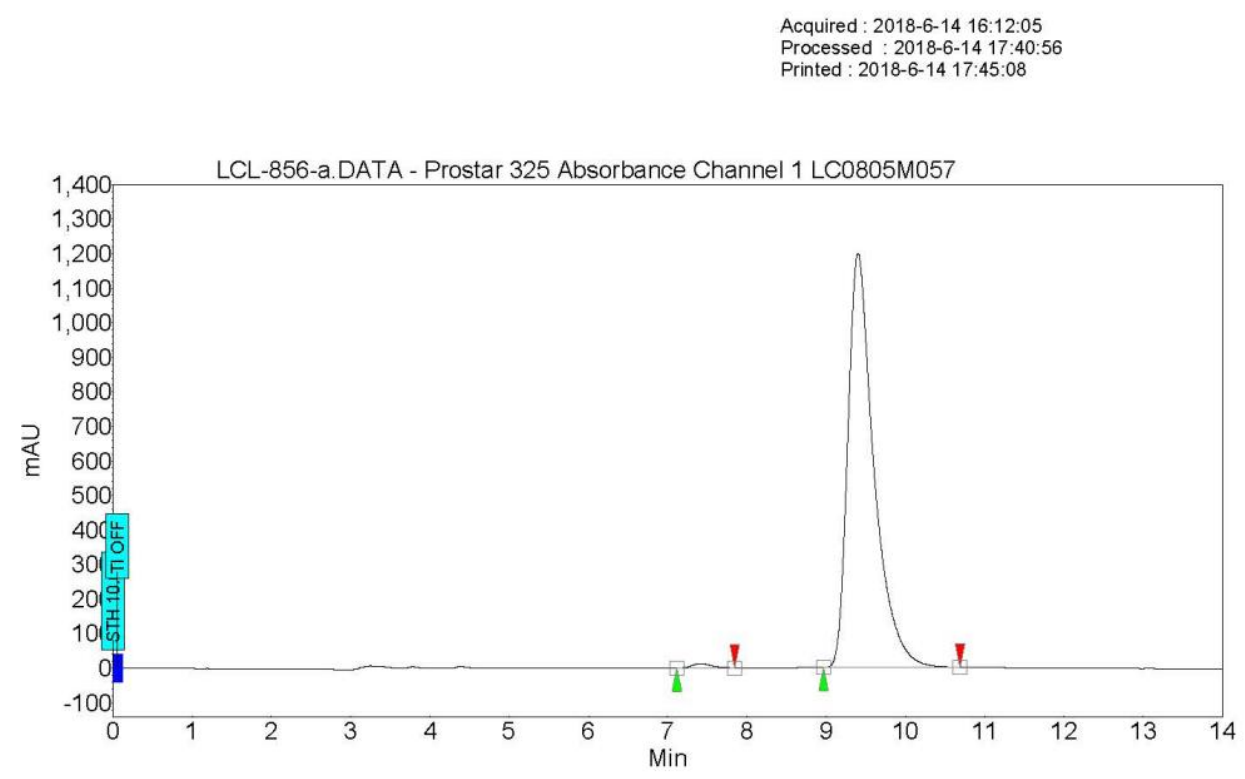

Peak results :

\begin{tabular}{|c|c|r|r|r|r|r|}
\hline Index & Name & $\begin{array}{l}\text { Time } \\
\text { [Min] }\end{array}$ & $\begin{array}{l}\text { Amount } \\
\text { [\% Area] }\end{array}$ & $\begin{array}{r}\text { Height } \\
\text { [mAU] }\end{array}$ & $\begin{array}{r}\text { Area } \\
\text { [mAU.Min] }\end{array}$ & $\begin{array}{r}\text { Area \% } \\
\text { [\%] }\end{array}$ \\
\hline \hline 1 & - & 7.42 & 0.78 & 12.5 & 3.6 & 0.744 \\
\hline 2 & - & 9.40 & 99.22 & 1198.4 & 456.4 & 99.216 \\
\hline & & & 100.00 & 1210.9 & 460.0 & 100.000 \\
\hline Total & & & 100 & \\
\hline
\end{tabular}




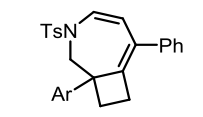

(士)-2c, $\mathrm{Ar}=4-\mathrm{MeOC}_{6} \mathrm{H}_{4}$

\section{Chromatogram : LCL-866-r_channel1}

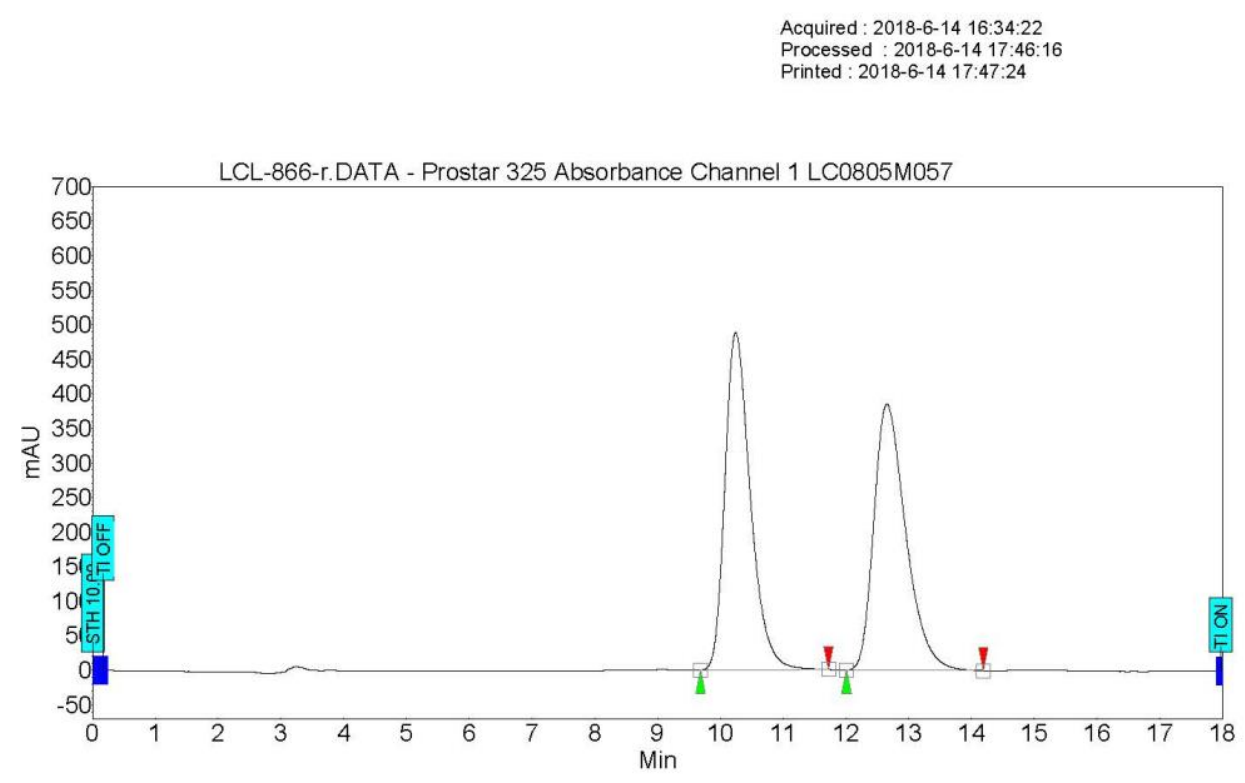

Peak results :

\begin{tabular}{|c|l|r|r|r|r|r|}
\hline Index & Name & $\begin{array}{r}\text { Time } \\
\text { [Min] }\end{array}$ & $\begin{array}{r}\text { Amount } \\
\text { [\% Area] }\end{array}$ & $\begin{array}{r}\text { Height } \\
\text { [mAU] }\end{array}$ & $\begin{array}{r}\text { Area } \\
\text { [mAU.Min] }\end{array}$ & $\begin{array}{r}\text { Area \% } \\
{[\%]}\end{array}$ \\
\hline \hline 1 & - & 10.25 & 50.26 & 488.8 & 229.5 & 50.255 \\
\hline 2 & - & 12.65 & 49.74 & 385.3 & 227.2 & 49.745 \\
\hline & & & & & & \\
\hline Total & & & 100.00 & 874.1 & 456.7 & 100.000 \\
\hline
\end{tabular}




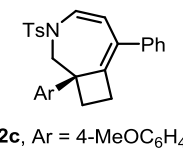

Chromatogram : LCL-866-a_channel1

Acquired : 2018-6-14 16:55:49

Printed : 2018-6-14 17:52:09

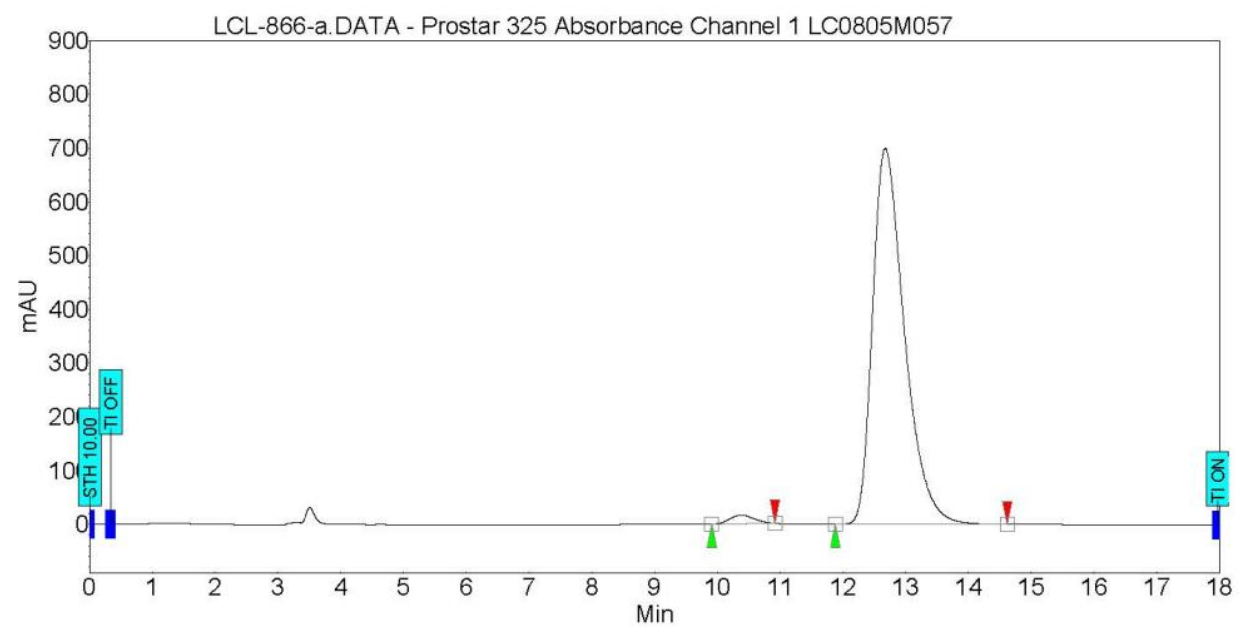

Peak results :

\begin{tabular}{|c|r|r|r|r|r|r|}
\hline Index & Name & $\begin{array}{r}\text { Time } \\
\text { [Min] }\end{array}$ & $\begin{array}{r}\text { Amount } \\
{[\% \text { Area] }}\end{array}$ & $\begin{array}{r}\text { Height } \\
\text { [mAU] }\end{array}$ & $\begin{array}{r}\text { Area } \\
\text { [mAU.Min] }\end{array}$ & $\begin{array}{r}\text { Area \% } \\
{[\%]}\end{array}$ \\
\hline \hline 1 & - & 10.37 & 1.72 & 16.0 & 7.2 & 1.724 \\
\hline 2 & - & 12.67 & 98.28 & 699.3 & 411.4 & 98.276 \\
\hline & & & & & & \\
\hline Total & & & 100.00 & 715.3 & 418.6 & 100.000 \\
\hline
\end{tabular}




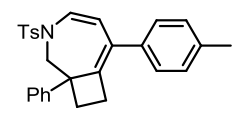

(士)-2e

Chromatogram : LCL-871-r-ADH_channel1

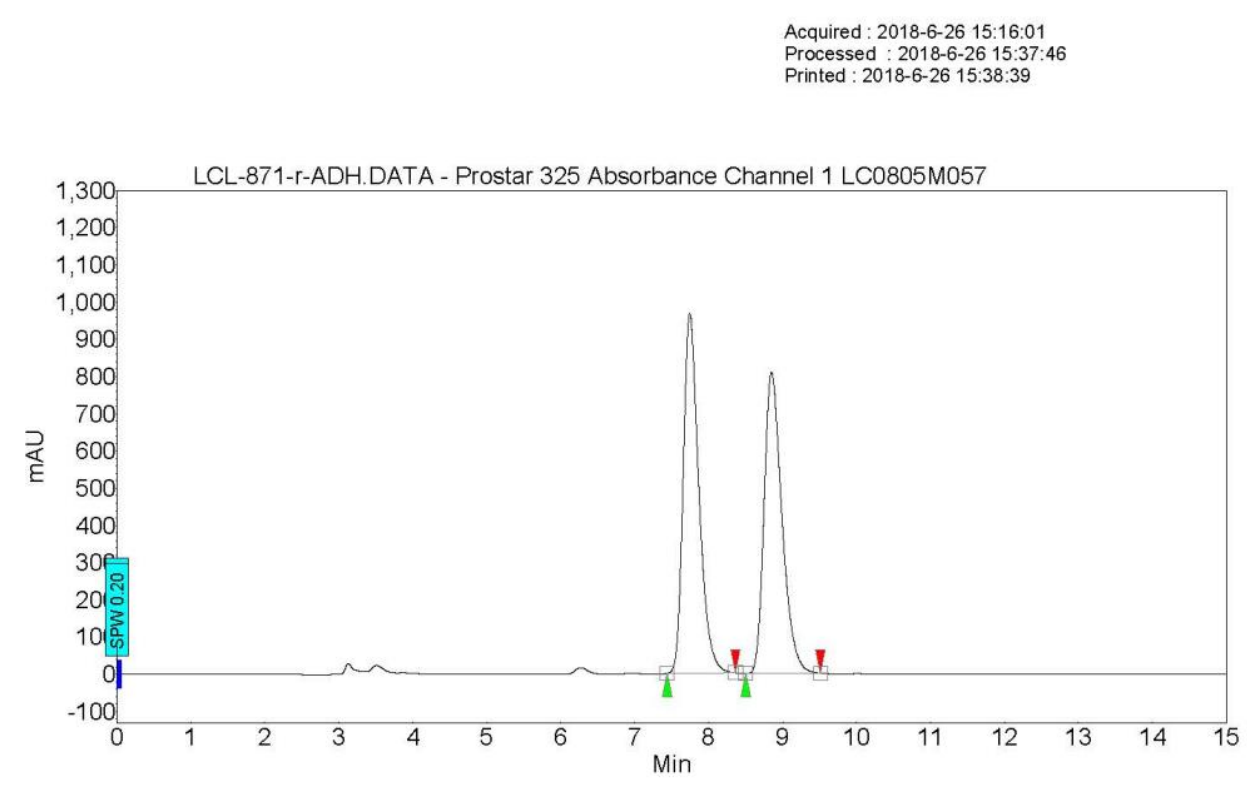

Peak results :

\begin{tabular}{|c|l|r|r|r|r|r|}
\hline Index & Name & $\begin{array}{r}\text { Time } \\
{[\text { Min] }}\end{array}$ & $\begin{array}{r}\text { Amount } \\
{[\% \text { Area] }}\end{array}$ & $\begin{array}{r}\text { Height } \\
{[\mathrm{mAU}]}\end{array}$ & $\begin{array}{r}\text { Area } \\
\text { [mAU.Min] }\end{array}$ & $\begin{array}{r}\text { Area \% } \\
{[\%]}\end{array}$ \\
\hline \hline 1 & - & 7.75 & 51.06 & 967.5 & 236.4 & 51.063 \\
\hline 2 & - & 8.85 & 48.94 & 809.7 & 226.6 & 48.937 \\
\hline & & & & & & \\
\hline Total & & & 100.00 & 1777.1 & 463.0 & 100.000 \\
\hline
\end{tabular}




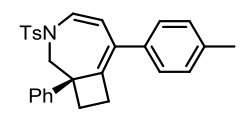

$2 e$

Chromatogram : LCL-871-a-ADH_channel1

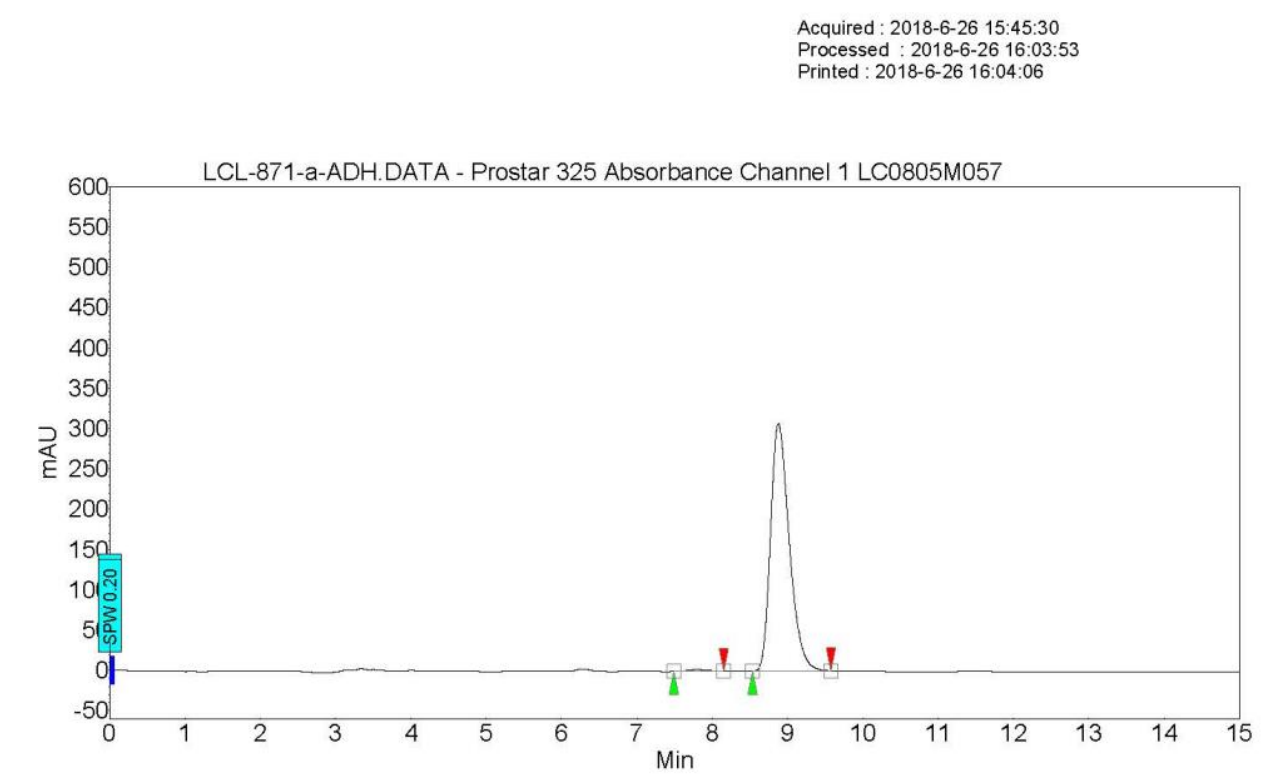

Peak results :

\begin{tabular}{|c|r|r|r|r|r|r|}
\hline Index & Name & $\begin{array}{r}\text { Time } \\
\text { [Min] }\end{array}$ & $\begin{array}{r}\text { Amount } \\
\text { [\% Area] }\end{array}$ & $\begin{array}{r}\text { Height } \\
\text { [mAU] }\end{array}$ & $\begin{array}{r}\text { Area } \\
\text { [mAU.Min] }\end{array}$ & $\begin{array}{r}\text { Area \% } \\
\text { [\%] }\end{array}$ \\
\hline \hline 1 & - & 7.79 & 0.75 & 2.2 & 0.7 & 0.753 \\
\hline 2 & - & 8.88 & 99.25 & 307.2 & 88.4 & 99.247 \\
\hline & & & & & & \\
\hline Total & & & 100.00 & 309.3 & 89.0 & 100.000 \\
\hline
\end{tabular}




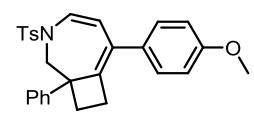

(士)-2f

Chromatogram : LCL-872-r-ADH_channel1

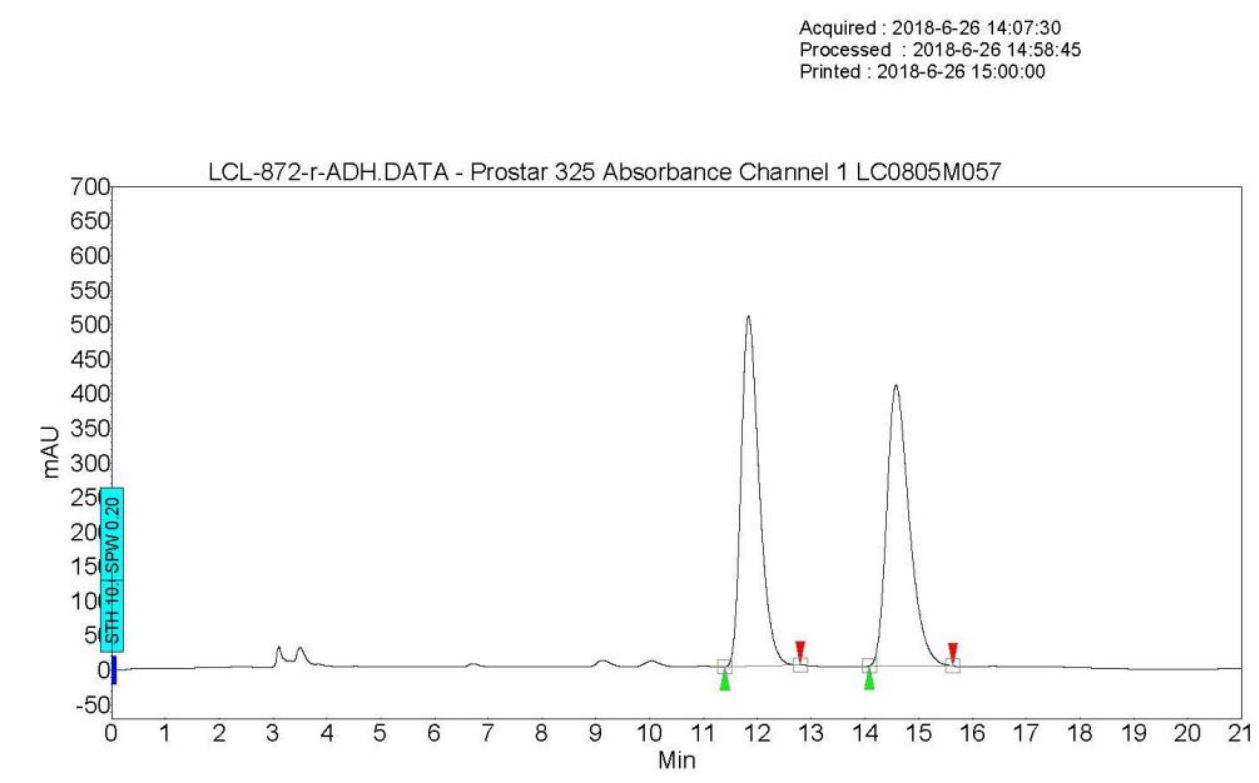

Peak results :

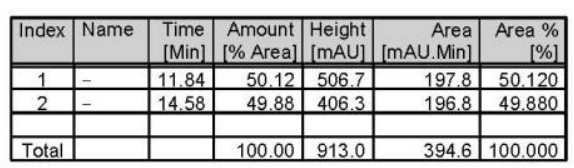

Page 1/1 


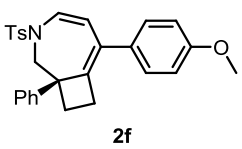

Chromatogram : LCL-872-a-ADH_channel1

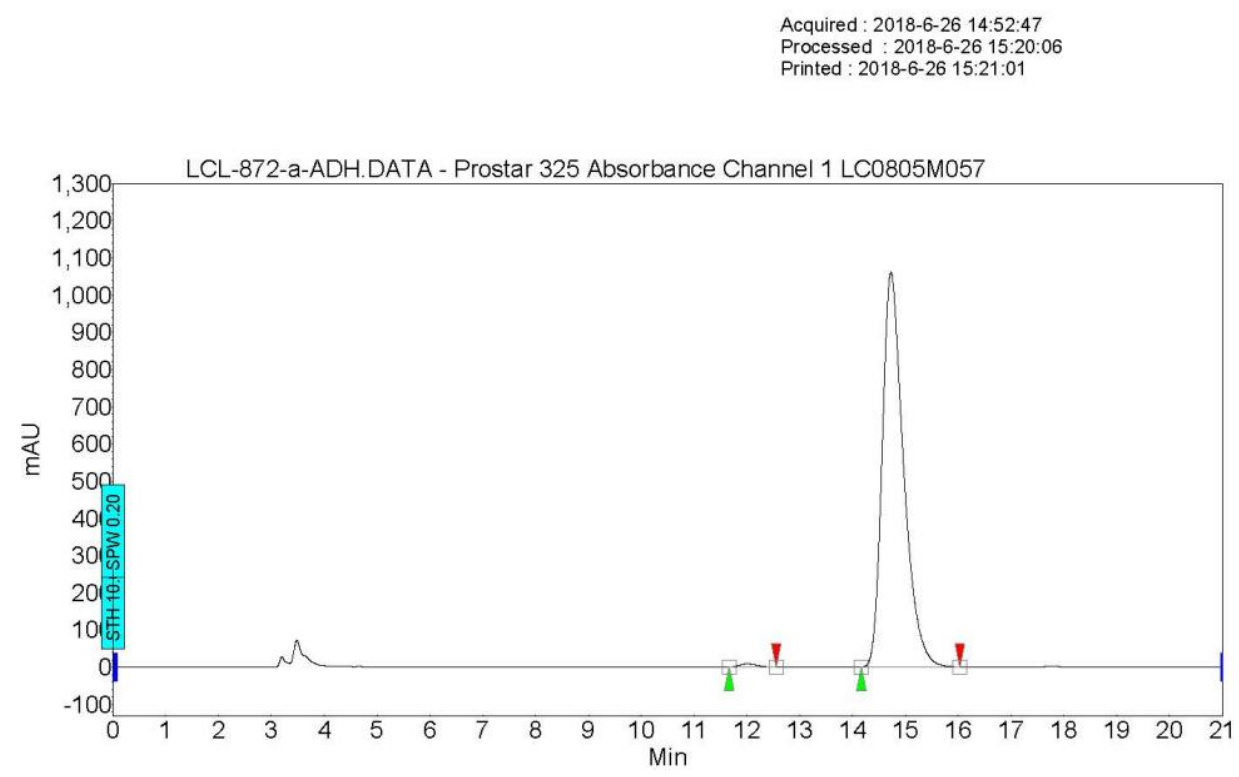

Peak results :

\begin{tabular}{|c|c|r|r|r|r|r|}
\hline Index & Name & $\begin{array}{l}\text { Time } \\
\text { [Min] }\end{array}$ & $\begin{array}{r}\text { Amount } \\
\text { [\% Area] }\end{array}$ & $\begin{array}{r}\text { Height } \\
\text { [mAU] }\end{array}$ & $\begin{array}{r}\text { Area } \\
\text { [mAU.Min] }\end{array}$ & $\begin{array}{r}\text { Area \% } \\
\text { [\%] }\end{array}$ \\
\hline \hline 1 & - & 12.01 & 0.67 & 9.0 & 3.4 & 0.673 \\
\hline 2 & - & 14.73 & 99.33 & 1061.0 & 496.7 & 99.327 \\
\hline & & & & & & \\
\hline Total & & & 100.00 & 1070.0 & 500.1 & 100.000 \\
\hline
\end{tabular}




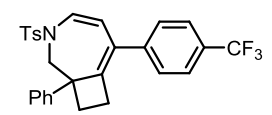

(士)-2g

Chromatogram : LCL-864-r_channel1

Acquired : 2018-6-14 15:15:23

Printed : 2018-6-14 16:22:06

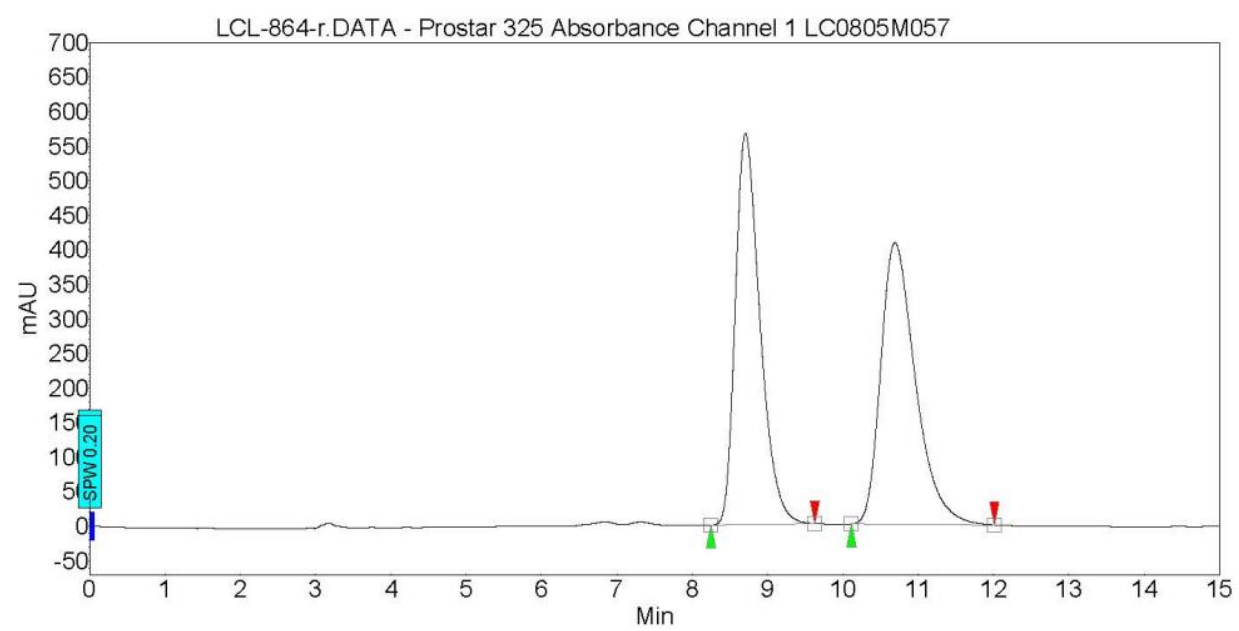

Peak results :

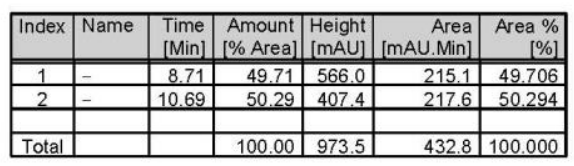

Page $1 / 1$ 


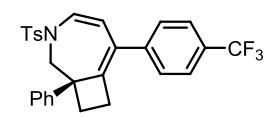

$2 \mathrm{~g}$

Chromatogram : LCL-864-a_channel1

Acquired : 2018-6-14 15:33:45

- $16: 29: 37$

Printed : 2018-6-14 16:29:37

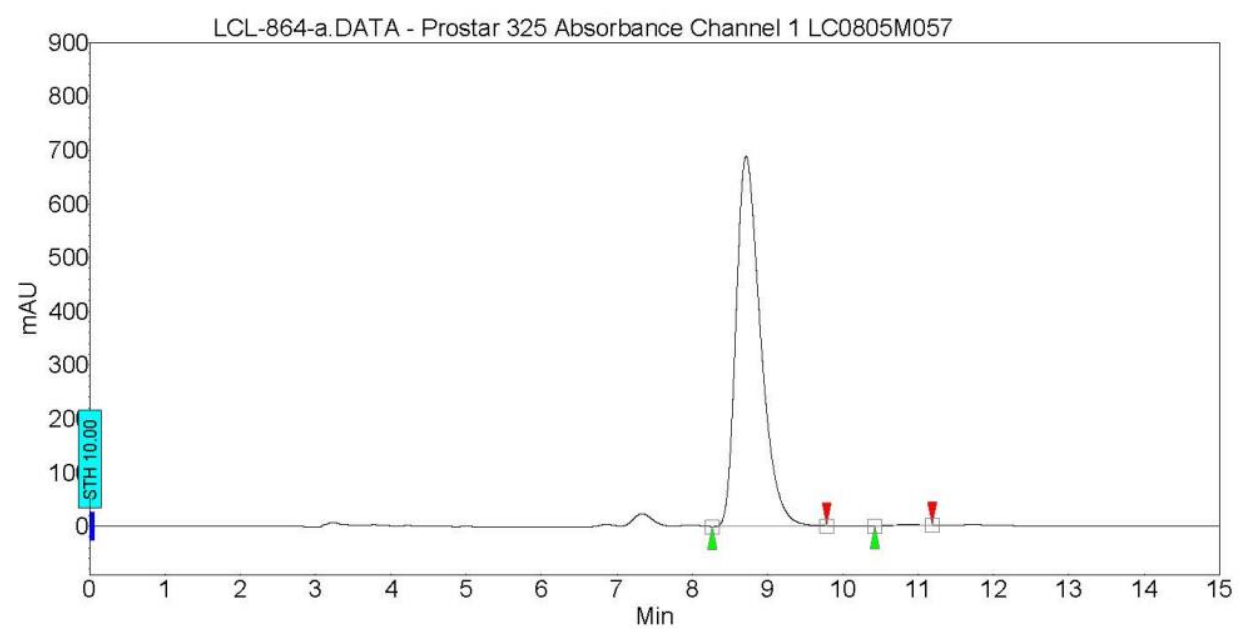

Peak results :

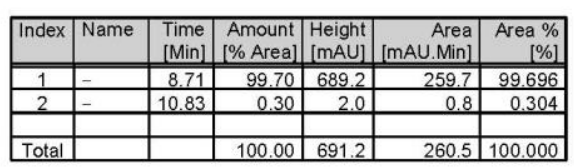

Page $1 / 1$ 


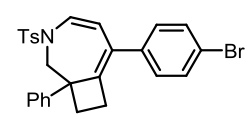

(士)-2h

Chromatogram : LCL-891-r-ADH_channel1

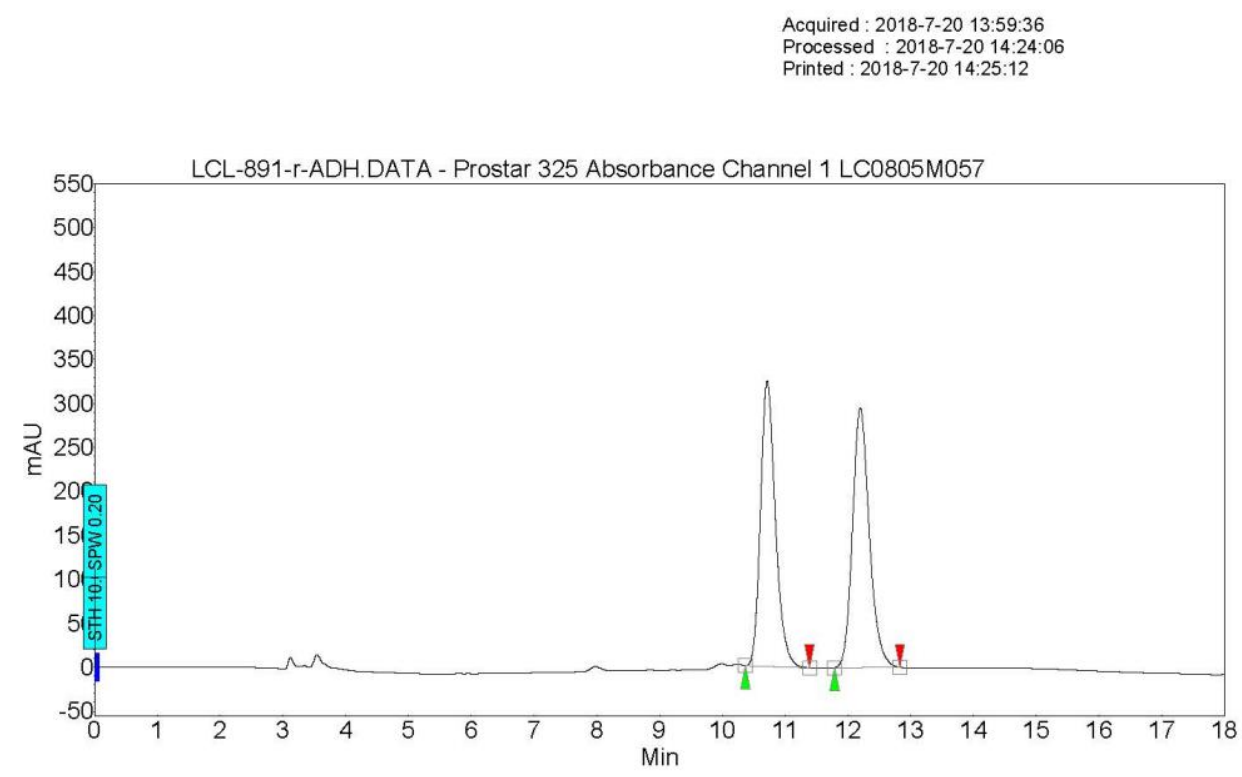

Peak results :

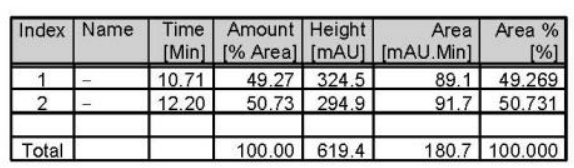

Page 1/1 


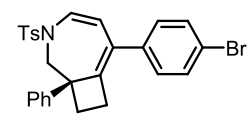

2h

Chromatogram : LCL-891-a-ADH_channel1

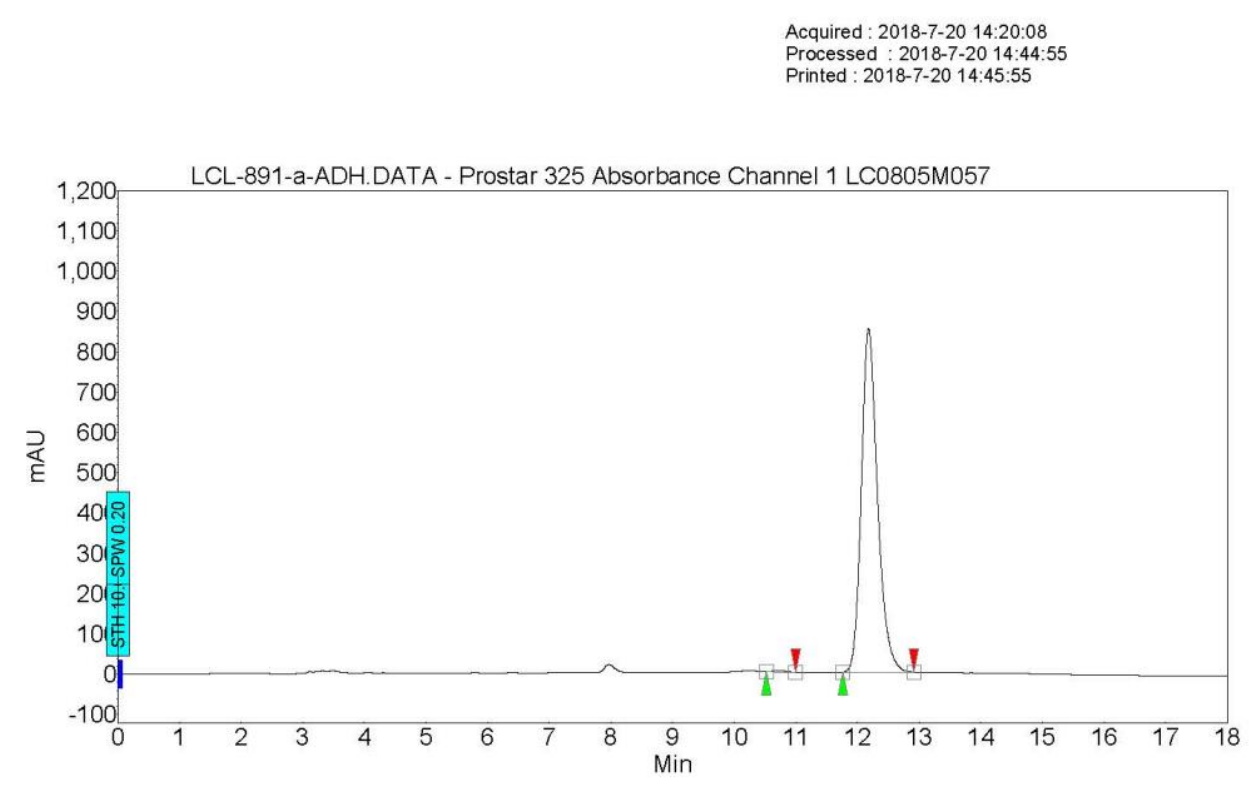

Peak results :

\begin{tabular}{|c|l|r|r|r|r|r|}
\hline Index & Name & $\begin{array}{r}\text { Time } \\
\text { [Min] }\end{array}$ & $\begin{array}{r}\text { Amount } \\
\text { [\% Area] }\end{array}$ & $\begin{array}{r}\text { Height } \\
\text { [mAU] }\end{array}$ & $\begin{array}{r}\text { Area } \\
\text { [mAU.Min] }\end{array}$ & $\begin{array}{r}\text { Area \% } \\
{[\%]}\end{array}$ \\
\hline \hline 2 & - & 10.72 & 0.41 & 4.6 & 1.1 & 0.405 \\
\hline 1 & - & 12.18 & 99.59 & 852.9 & 259.4 & 99.595 \\
\hline & & & & & & \\
\hline Total & & & 100.00 & 857.5 & 260.5 & 100.000 \\
\hline
\end{tabular}




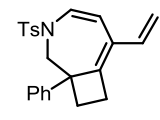

(士)-2i

Chromatogram : LCL-875-r-0.5_channel1

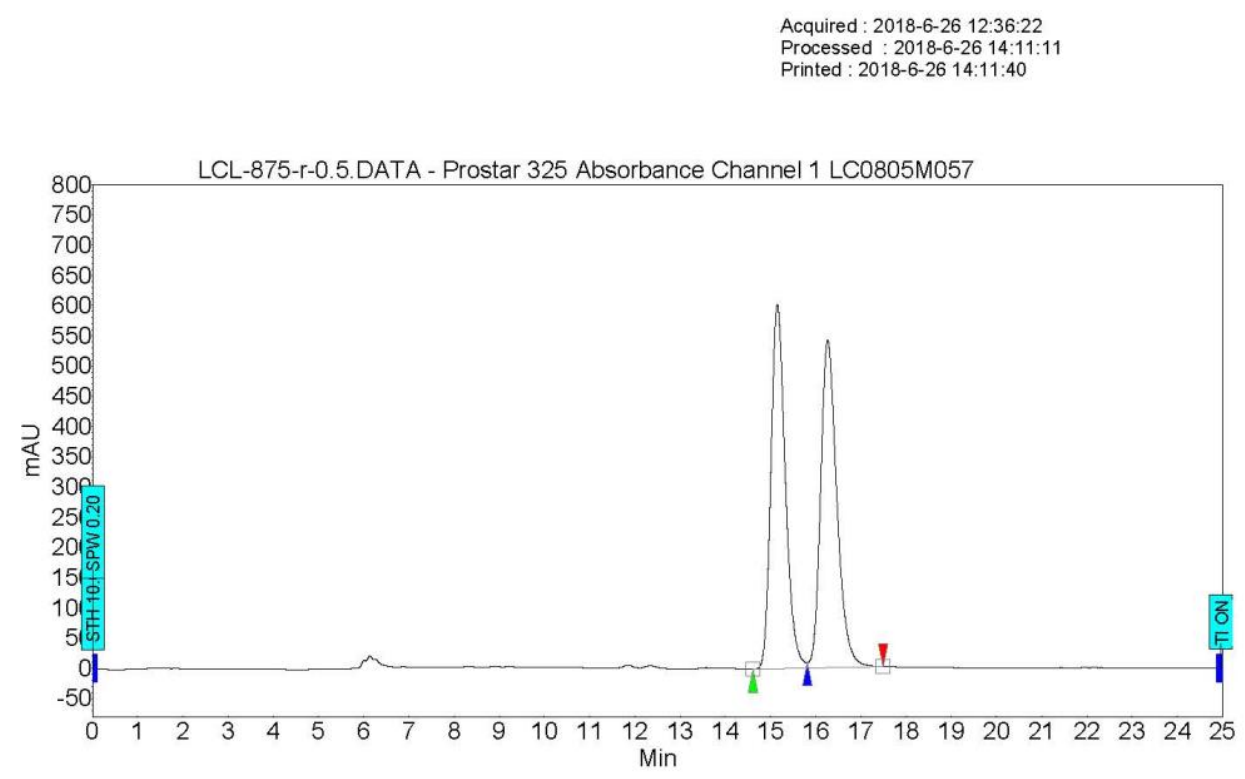

Peak results :

\begin{tabular}{|c|c|r|r|r|r|r|}
\hline Index & Name & $\begin{array}{r}\text { Time } \\
\text { [Min] }\end{array}$ & $\begin{array}{r}\text { Amount } \\
\text { [\% Area] }\end{array}$ & $\begin{array}{r}\text { Height } \\
\text { [mAU] }\end{array}$ & $\begin{array}{r}\text { Area } \\
\text { [mAU.Min] }\end{array}$ & $\begin{array}{r}\text { Area \% } \\
\text { [\%] }\end{array}$ \\
\hline \hline 1 & - & 15.15 & 49.69 & 602.3 & 222.6 & 49.685 \\
\hline 2 & - & 16.27 & 50.31 & 541.5 & 225.5 & 50.315 \\
\hline & & & & & & \\
\hline Total & & & 100.00 & 1143.8 & 448.1 & 100.000 \\
\hline
\end{tabular}




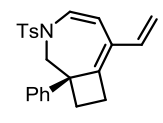

2i

Chromatogram : LCL-875-a-0.5_channel1

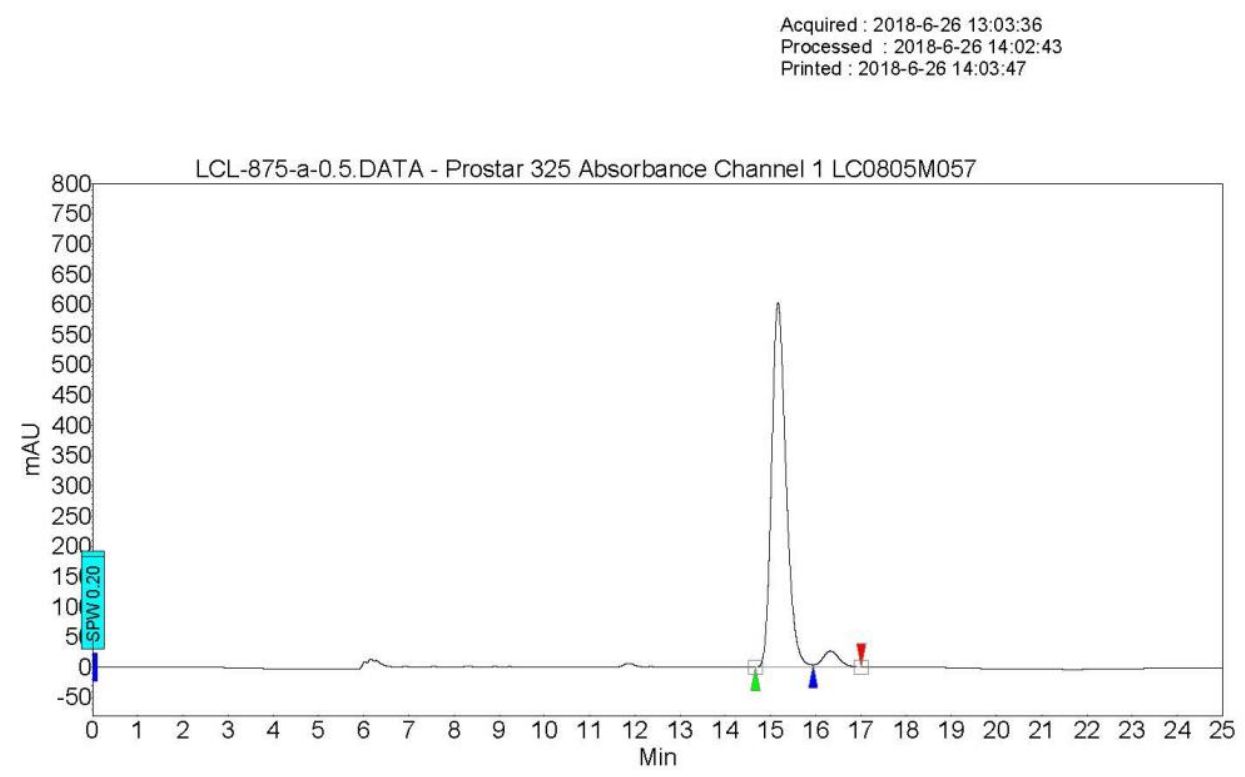

Peak results :

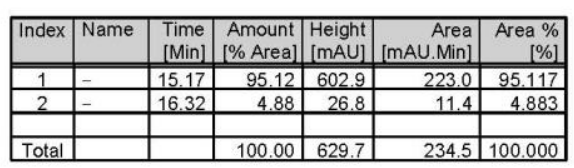




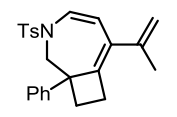

(士)-2j

Chromatogram : LCL-879-r-0.5_channel1

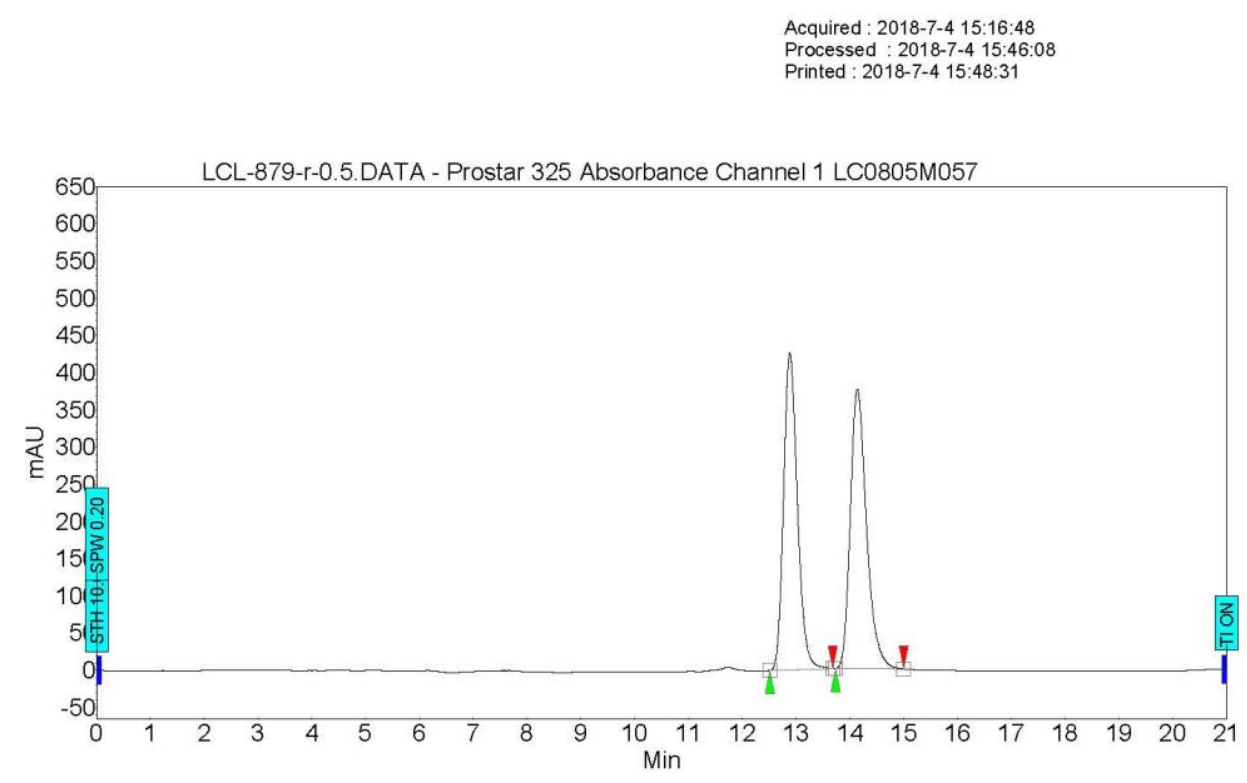

Peak results :

\begin{tabular}{|c|l|r|r|r|r|r|}
\hline Index & Name & $\begin{array}{r}\text { Time } \\
\text { [Min] }\end{array}$ & $\begin{array}{r}\text { Amount } \\
\text { [\% Area] }\end{array}$ & $\begin{array}{r}\text { Height } \\
\text { [mAU] }\end{array}$ & $\begin{array}{r}\text { Area } \\
\text { [mAU.Min] }\end{array}$ & $\begin{array}{r}\text { Area \% } \\
{[\%]}\end{array}$ \\
\hline \hline 1 & - & 12.89 & 49.15 & 425.9 & 126.6 & 49.147 \\
\hline 2 & - & 14.14 & 50.85 & 376.0 & 131.0 & 50.853 \\
\hline & & & & & & \\
\hline Total & & & 100.00 & 801.9 & 257.7 & 100.000 \\
\hline
\end{tabular}




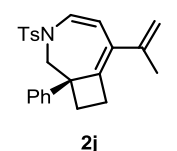

Chromatogram : LCL-879-a-0.5_channel1

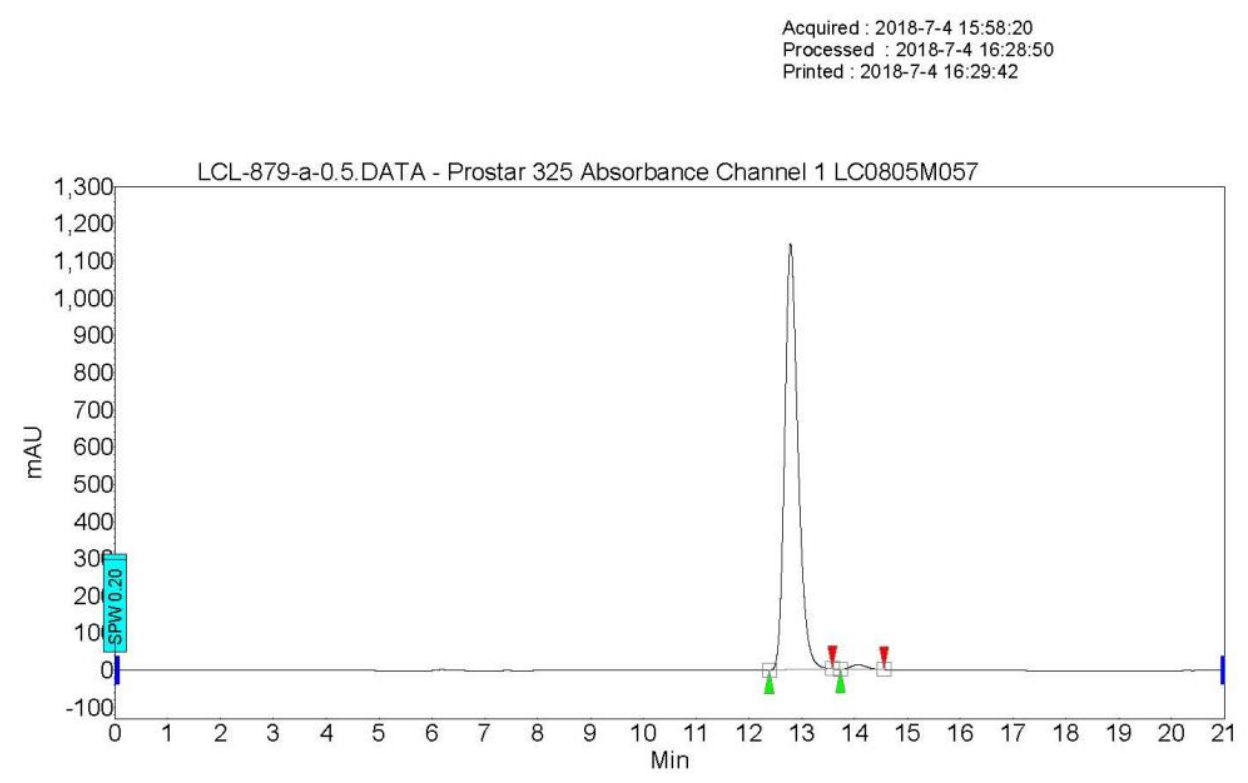

Peak results :

\begin{tabular}{|c|r|r|r|r|r|r|}
\hline Index & Name & $\begin{array}{r}\text { Time } \\
\text { [Min] }\end{array}$ & $\begin{array}{r}\text { Amount } \\
\text { [\% Area] }\end{array}$ & $\begin{array}{r}\text { Height } \\
\text { [mAU] }\end{array}$ & $\begin{array}{r}\text { Area } \\
\text { [mAU.Min] }\end{array}$ & $\begin{array}{r}\text { Area \% } \\
\text { \%] }\end{array}$ \\
\hline \hline 1 & - & 12.79 & 98.72 & 1145.0 & 332.7 & 98.722 \\
\hline 2 & - & 14.07 & 1.28 & 12.8 & 4.3 & 1.278 \\
\hline & & & & & & \\
\hline Total & & & 100.00 & 1157.9 & 337.0 & 100.000 \\
\hline
\end{tabular}




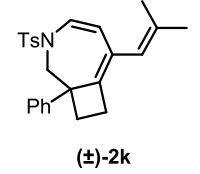

Chromatogram : LCL-893-r_channel1

Acquired : 2018-7-20 9:45:36

Processed : 2018-7-20 10:14:06
Printed : 2018-7-20 10:16:22

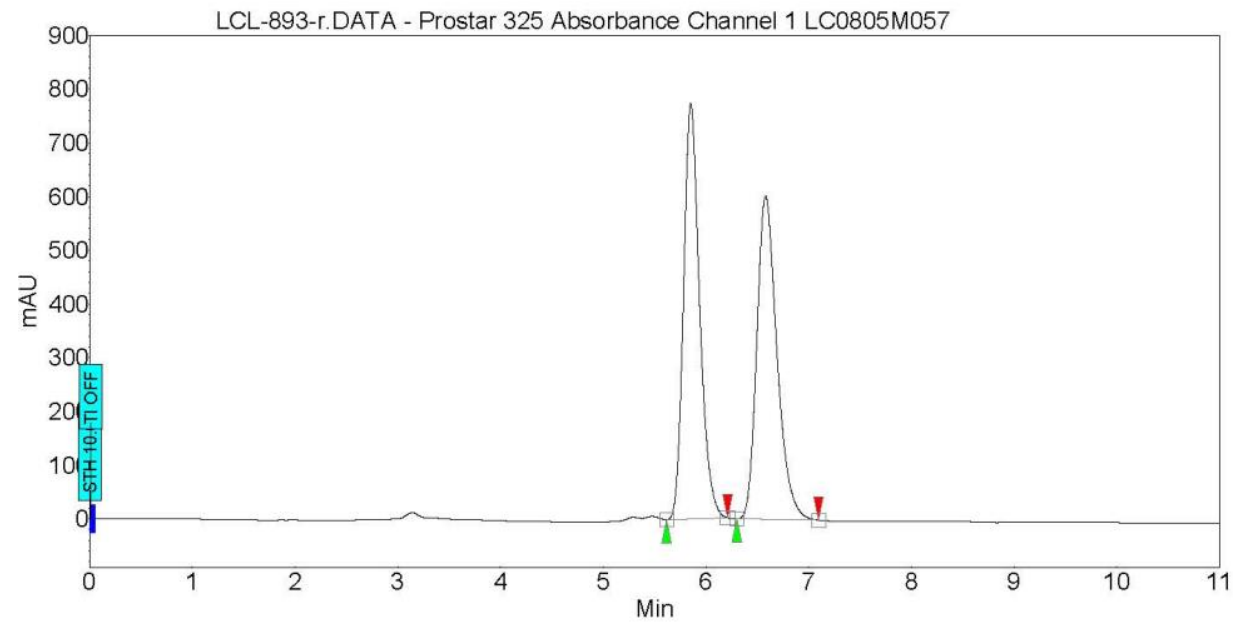

Peak results :

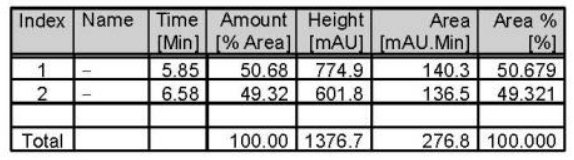

Page $1 / 1$ 


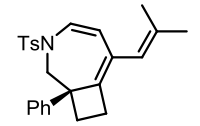

2k

Chromatogram : LCL-893-a_channel1

Acquired: 2018-7-20 10:11:40

Processed : 2018-7-20 10:29:54
Printed : 2018-7-20 10:30:58

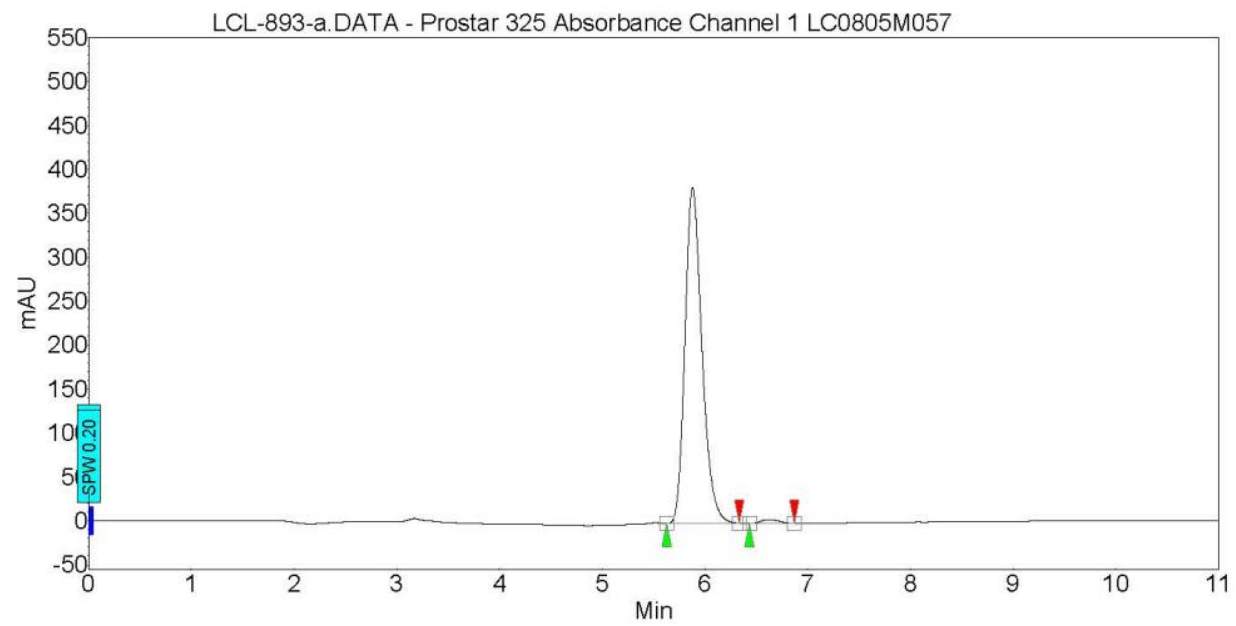

Peak results :

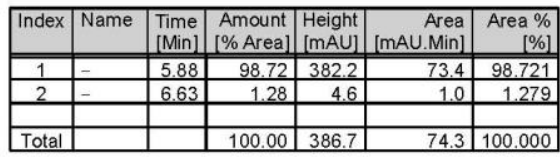

Page $1 / 1$ 


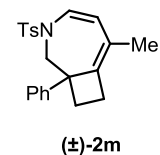

Chromatogram : LCL-925-2-r_channel1

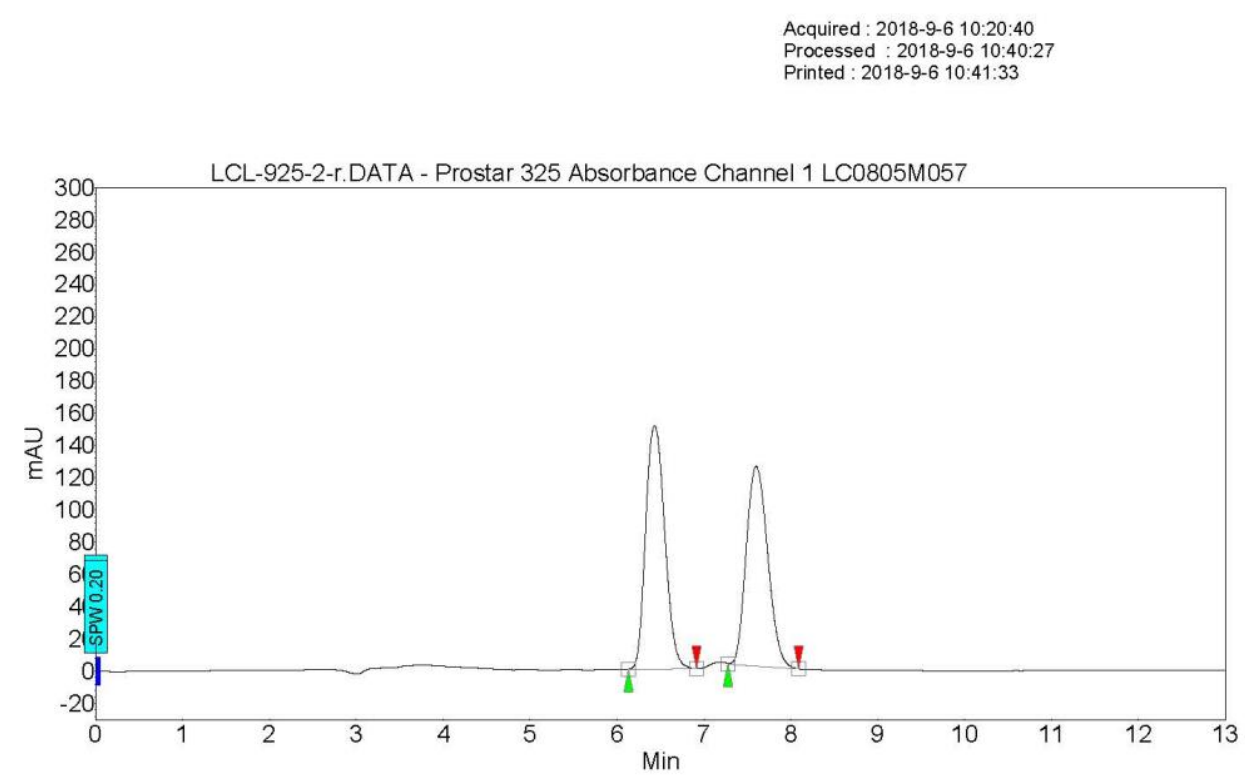

Peak results :

\begin{tabular}{|c|c|r|r|r|r|r|}
\hline Index & Name & $\begin{array}{r}\text { Time } \\
\text { [Min] }\end{array}$ & $\begin{array}{r}\text { Amount } \\
\text { [\% Area] }\end{array}$ & $\begin{array}{r}\text { Height } \\
\text { [mAU] }\end{array}$ & $\begin{array}{r}\text { Area } \\
\text { [mAU.Min] }\end{array}$ & $\begin{array}{r}\text { Area \% } \\
\text { [\%] }\end{array}$ \\
\hline \hline 1 & - & 6.43 & 51.92 & 150.9 & 38.4 & 51.917 \\
\hline 2 & - & 7.60 & 48.08 & 123.8 & 35.6 & 48.083 \\
\hline & & & & & & \\
\hline Total & & & 100.00 & 274.7 & 74.0 & 100.000 \\
\hline
\end{tabular}




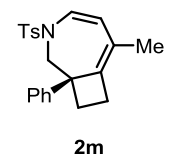

Chromatogram : LCL-925-2-a_channel1

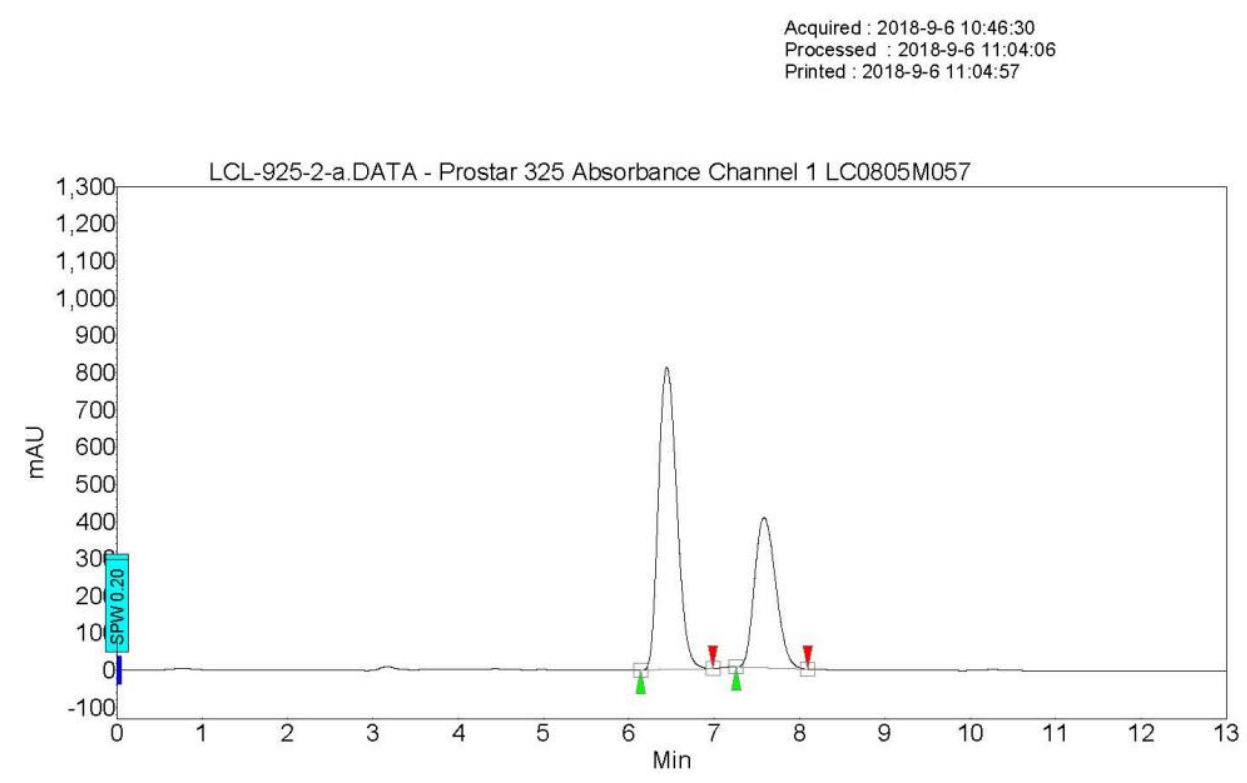

Peak results :

\begin{tabular}{|c|c|r|r|r|r|r|}
\hline Index & Name & $\begin{array}{r}\text { Time } \\
\text { [Min] }\end{array}$ & $\begin{array}{r}\text { Amount } \\
\text { [\% Area] }\end{array}$ & $\begin{array}{r}\text { Height } \\
\text { [mAU] }\end{array}$ & $\begin{array}{r}\text { Area } \\
\text { [mAU.Min] }\end{array}$ & $\begin{array}{r}\text { Area \% } \\
\text { [\%] }\end{array}$ \\
\hline \hline 1 & - & 6.45 & 63.54 & 8111.6 & 203.0 & 63.538 \\
\hline 2 & - & 7.59 & 36.46 & 403.8 & 116.5 & 36.462 \\
\hline & & & & & & \\
\hline Total & & & 100.00 & 1215.4 & 319.5 & 100.000 \\
\hline
\end{tabular}




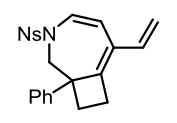

(士)-2n

\section{Chromatogram : LCL-892-r_channel1}

Acquired : 2018-7-20 14:50:29

Printed : 2018-7-20 15:22:15

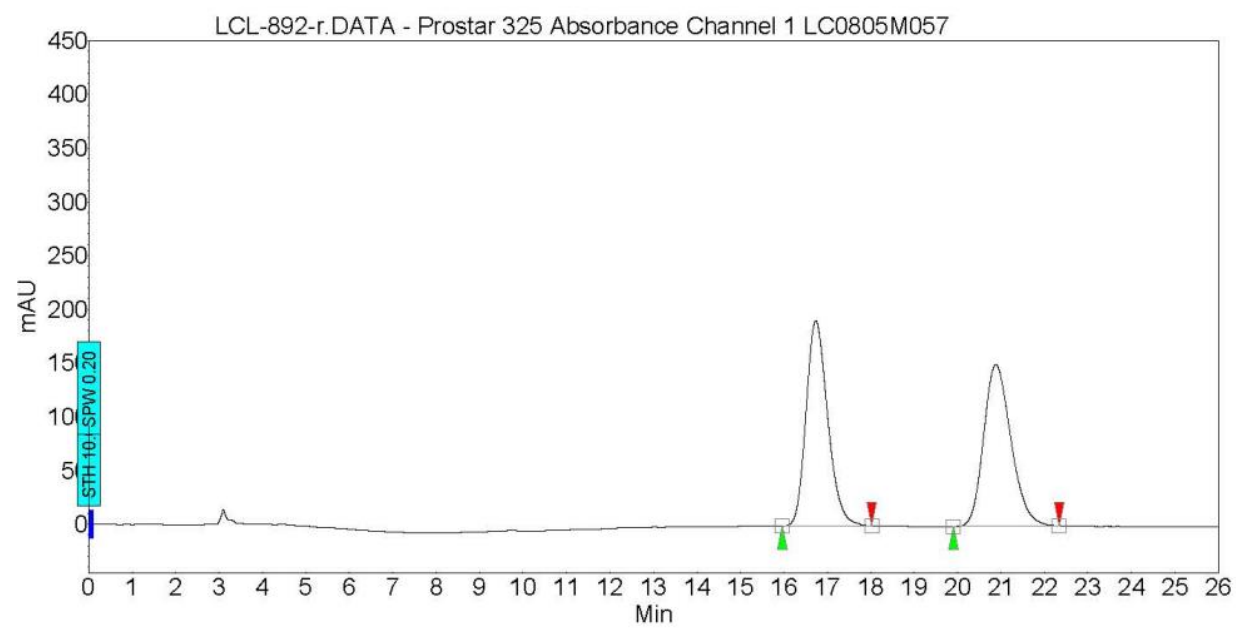

Peak results :

\begin{tabular}{|c|c|r|r|r|r|r|}
\hline Index & Name & $\begin{array}{r}\text { Time } \\
\text { [Min] }\end{array}$ & $\begin{array}{r}\text { Amount } \\
\text { [\% Area] }\end{array}$ & $\begin{array}{r}\text { Height } \\
\text { [mAU] }\end{array}$ & $\begin{array}{r}\text { Area } \\
\text { [mAU.Min] }\end{array}$ & $\begin{array}{r}\text { Area \% } \\
{[\%]}\end{array}$ \\
\hline \hline 1 & - & 16.73 & 50.27 & 190.9 & 110.5 & 50.272 \\
\hline 2 & - & 20.87 & 49.73 & 150.3 & 109.3 & 49.728 \\
\hline & & & & & & \\
\hline Total & & & 100.00 & 341.1 & 219.8 & 100.000 \\
\hline
\end{tabular}




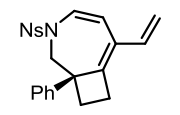

2n

Chromatogram : LCL-892-a_channel1

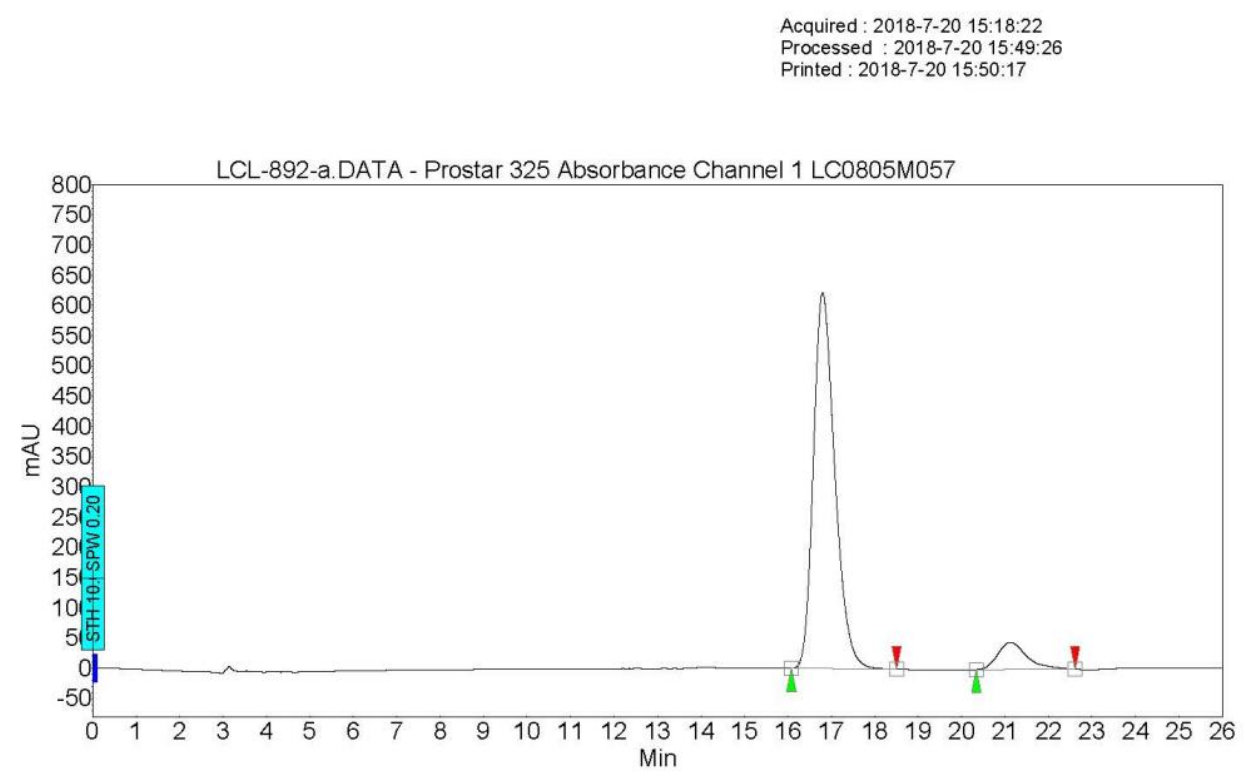

Peak results :

\begin{tabular}{|c|l|r|r|r|r|r|}
\hline Index & Name & $\begin{array}{r}\text { Time } \\
\text { [Min] }\end{array}$ & $\begin{array}{r}\text { Amount } \\
\text { [\% Area] }\end{array}$ & $\begin{array}{r}\text { Height } \\
\text { [mAU] }\end{array}$ & $\begin{array}{r}\text { Area } \\
\text { [mAU.Min] }\end{array}$ & $\begin{array}{r}\text { Area \% } \\
{[\%]}\end{array}$ \\
\hline \hline 1 & - & 16.80 & 91.01 & 621.7 & 355.0 & 91.010 \\
\hline 2 & - & 21.11 & 8.99 & 44.8 & 35.1 & 8.990 \\
\hline & & & & & & \\
\hline Total & & & 100.00 & 666.5 & 390.1 & 100.000 \\
\hline
\end{tabular}


Dr

(士)-2o, $\mathrm{Ar}=4-\mathrm{MeOC}_{6} \mathrm{H}_{4}$

Chromatogram : LCL-924-r-ADH_channel1

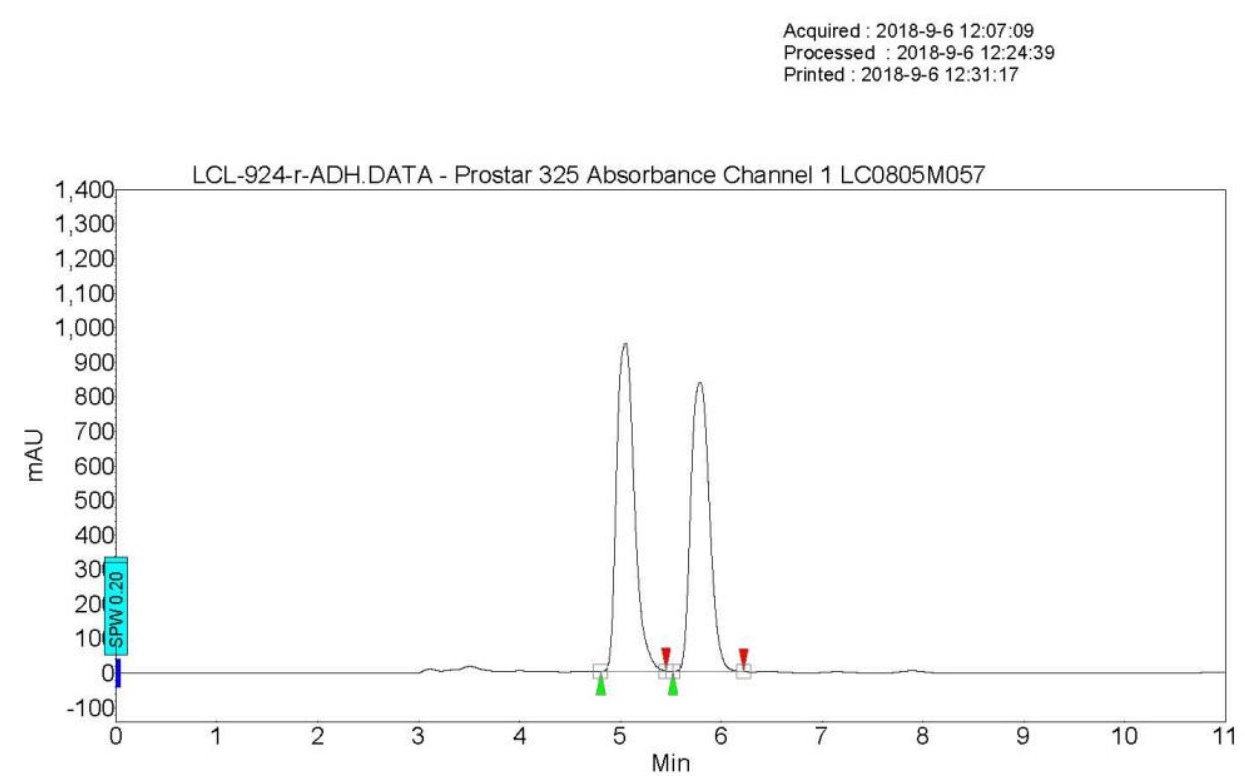

Peak results :

\begin{tabular}{|c|c|c|c|c|c|c|}
\hline Index & Name & $\begin{array}{l}\text { Time } \\
\text { [Min] }\end{array}$ & $\begin{array}{l}\text { Amount } \\
\text { [\% Areal }\end{array}$ & $\begin{array}{l}\text { Height } \\
\text { [mAU] }\end{array}$ & $\begin{array}{r}\text { Area } \\
\text { [mAU.Min] }\end{array}$ & $\begin{array}{r}\text { Area \% } \\
{[\%]}\end{array}$ \\
\hline 1 & & 5.05 & 51.99 & 9950.3 & 193.9 & 51.990 \\
\hline 2 & & 5.79 & 48.01 & 837.0 & 179.1 & 48.010 \\
\hline total & & & 100.00 & 118873 & & 0000 \\
\hline
\end{tabular}




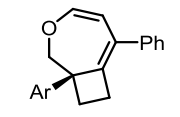

2o, $\mathrm{Ar}=4-\mathrm{MeOC}_{6} \mathrm{H}_{4}$

\section{Chromatogram : LCL-924-a-ADH_channel1}

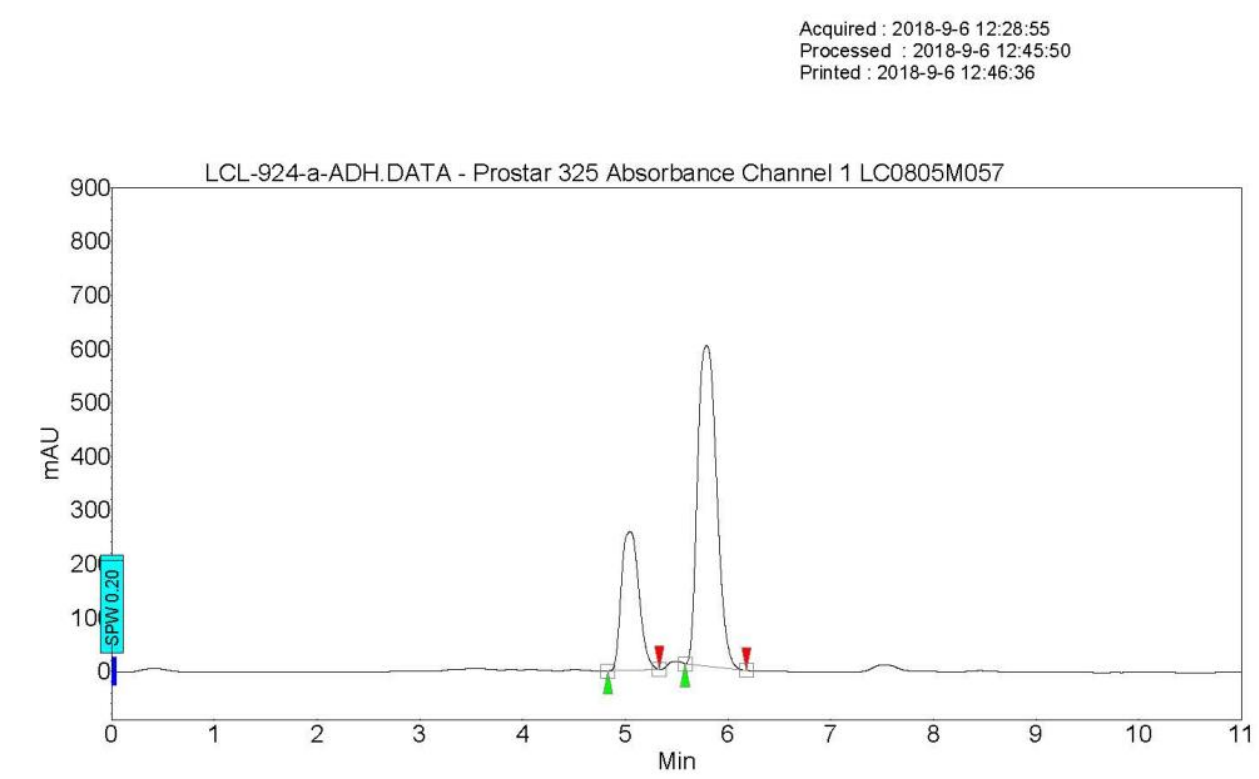

Peak results :

\begin{tabular}{|c|r|r|r|r|r|r|}
\hline Index & Name & $\begin{array}{r}\text { Time } \\
\text { [Min] }\end{array}$ & $\begin{array}{r}\text { Amount } \\
\text { [\% Area] }\end{array}$ & $\begin{array}{r}\text { Height } \\
\text { [mAU] }\end{array}$ & $\begin{array}{r}\text { Area } \\
\text { [mAU.Min] }\end{array}$ & $\begin{array}{r}\text { Area \% } \\
\text { [\%] }\end{array}$ \\
\hline \hline 1 & - & 5.05 & 28.60 & 257.8 & 51.3 & 28.601 \\
\hline 2 & - & 5.79 & 71.40 & 596.4 & 128.0 & 71.399 \\
\hline & & & & & & \\
\hline Total & & & 100.00 & 854.2 & 179.3 & 100.000 \\
\hline
\end{tabular}




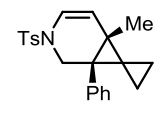

(士)-3m

Chromatogram : LCL-925-1-r-0.5_channel1

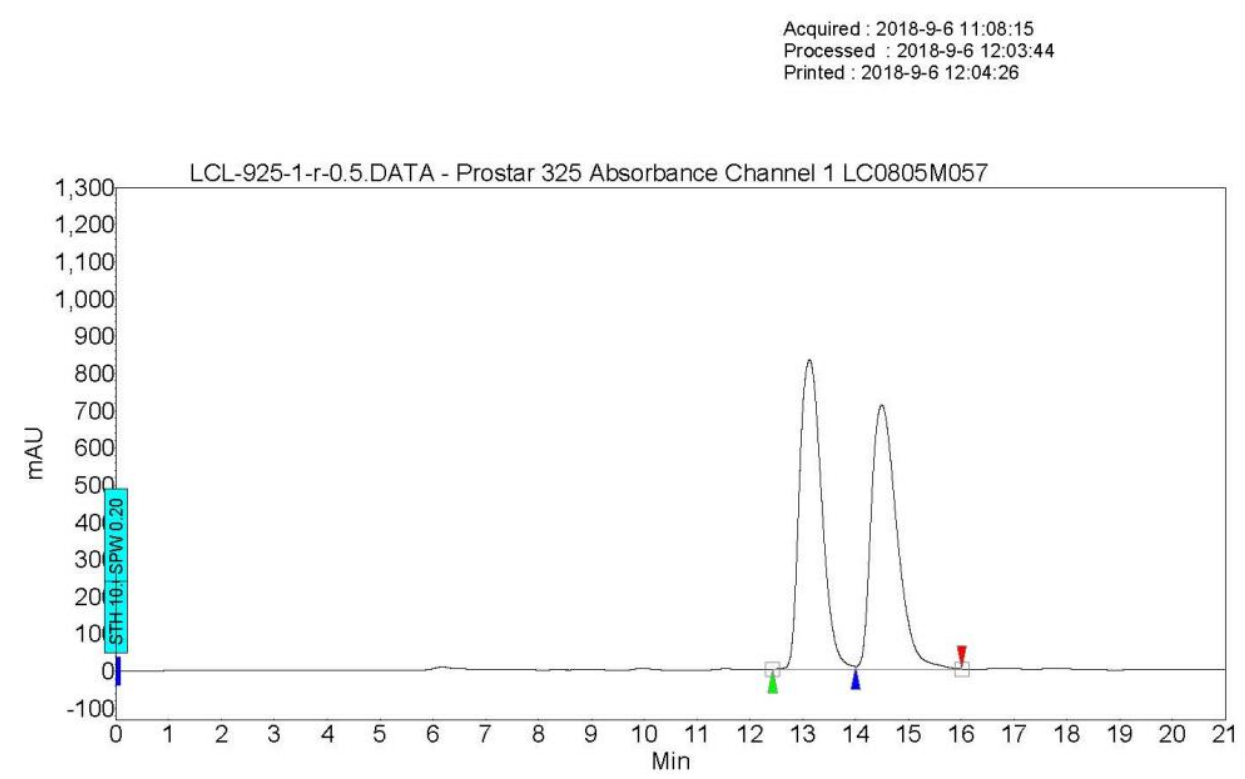

Peak results :

\begin{tabular}{|c|r|r|r|r|r|r|}
\hline Index & Name & $\begin{array}{r}\text { Time } \\
\text { [Min] }\end{array}$ & $\begin{array}{r}\text { Amount } \\
\text { [\% Area] }\end{array}$ & $\begin{array}{r}\text { Height } \\
\text { [mAU] }\end{array}$ & $\begin{array}{r}\text { Area } \\
\text { [mAU.Min] }\end{array}$ & $\begin{array}{r}\text { Area \% } \\
\text { [\%] }\end{array}$ \\
\hline \hline 1 & - & 13.13 & 50.28 & 831.3 & 404.6 & 50.281 \\
\hline 2 & - & 14.49 & 49.72 & 710.2 & 400.1 & 49.719 \\
\hline & & & & & & \\
\hline Total & & & 100.00 & 1541.5 & 804.7 & 100.000 \\
\hline
\end{tabular}




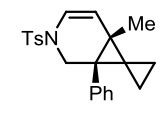

$3 \mathrm{~m}$

Chromatogram : LCL-925-1-a-0.5_channel1

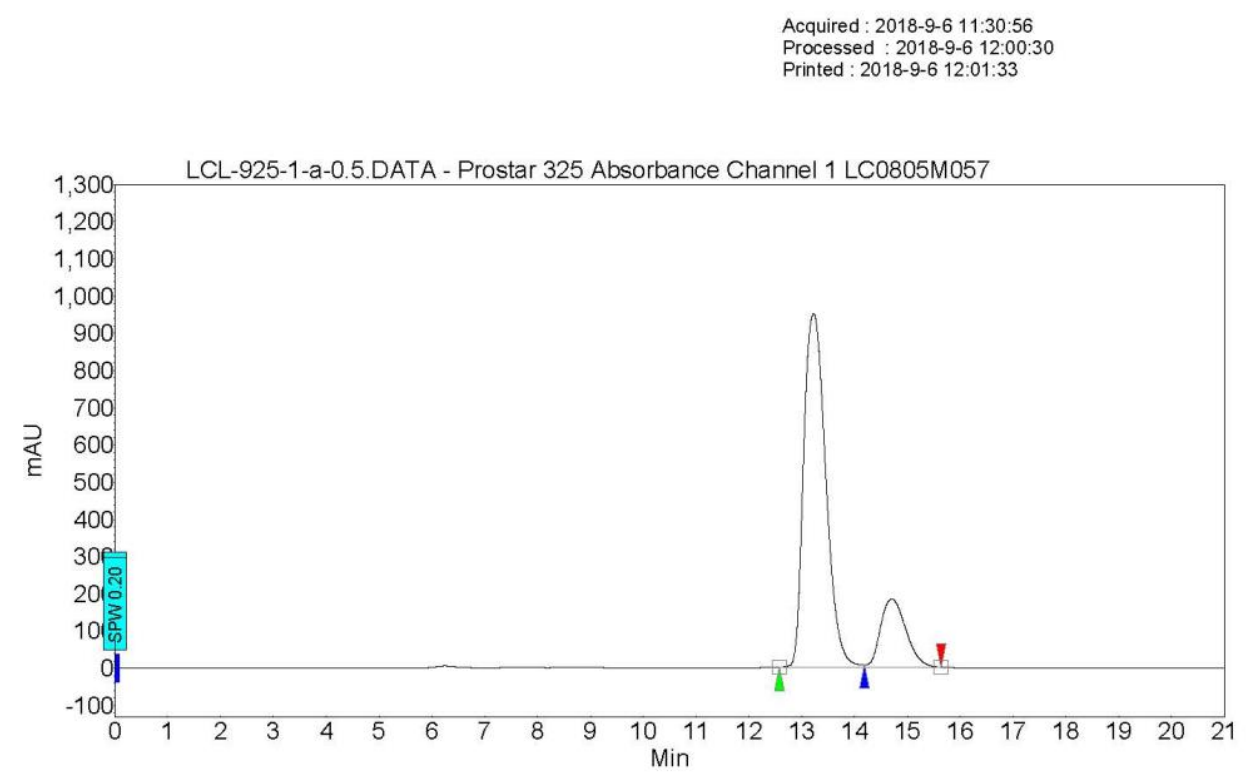

Peak results :

\begin{tabular}{|c|l|r|r|r|r|r|}
\hline Index & Name & $\begin{array}{r}\text { Time } \\
\text { [Min] }\end{array}$ & $\begin{array}{r}\text { Amount } \\
\text { [\% Area] }\end{array}$ & $\begin{array}{r}\text { Height } \\
\text { [mAU] }\end{array}$ & $\begin{array}{r}\text { Area } \\
\text { [mAU.Min] }\end{array}$ & $\begin{array}{r}\text { Area \% } \\
\text { [\%] }\end{array}$ \\
\hline \hline 1 & - & 13.23 & 81.86 & 950.5 & 460.3 & 81.864 \\
\hline 2 & - & 14.71 & 18.14 & 183.0 & 102.0 & 18.136 \\
\hline & & & & & & \\
\hline Total & & & 100.00 & 1133.5 & 562.3 & 100.000 \\
\hline
\end{tabular}

